International Journal of Bifurcation and Chaos, Vol. 16, No. 4 (2006) 775-858

(C) World Scientific Publishing Company

\title{
GENERATING MULTISCROLL CHAOTIC ATTRACTORS: THEORIES, METHODS AND APPLICATIONS
}

\author{
JINHU LÜ \\ The Key Laboratory of Systems and Control, \\ Institute of Systems Science, \\ Academy of Mathematics and Systems Science, \\ Chinese Academy of Sciences, Beijing 100080, P. R. China \\ jhlu@iss.ac.cn \\ GUANRONG CHEN \\ Department of Electronic Engineering, \\ City University of Hong Kong, Hong Kong SAR, P. R. China \\ gchen@ee.cityu.edu.hk
}

Received February 18, 2005; Revised March 17, 2005

\begin{abstract}
Over the last two decades, theoretical design and circuit implementation of various chaos generators have been a focal subject of increasing interest due to their promising applications in various real-world chaos-based technologies and information systems. In particular, generating complex multiscroll chaotic attractors via simple electronic circuits has seen rapid development. This article offers an overview of the subject on multiscroll chaotic attractors generation, including some fundamental theories, design methodologies, circuit implementations and practical applications. More precisely, the article first describes some effective design methods using piecewise-linear functions, cellular neural networks, nonlinear modulating functions, circuit component design, switching manifolds, multifolded tori formation, and so on. Based on different approaches, computer simulation and circuit implementation of various multiscroll chaotic attractors are then discussed in detail, with some theoretical proofs and laboratory experiments presented for verification and demonstration. It is then followed by some discussion on potential applications of multiscroll chaotic attractors, including secure and digital communications, synchronous prediction, random bit generation, and so on. The article is finally concluded with some future research outlooks, putting the important subject into engineering perspective.
\end{abstract}

Keywords: Chaos; Chua's circuit; cellular neural networks; hyperchaos; jerk circuit; multiscroll chaotic attractor; multifolded torus; nonlinear modulating function; switching manifold.

\section{Contents}

1. Introduction . . . . . . . . . . . . . . . . . . . . . . 777

2. Design of $n$-Scroll Chaotic Attractors via PWL Functions . . . . . . . . . . . . . 779

2.1. Design of $n$-double-scroll chaotic attractors using quasi-linear functions . . . . . 779

2.2. $n$-Scroll chaotic attractors from a generalized Chua's circuit . . . . . . . . . . . 782

2.3. $n$-Scroll chaotic attractors from the unfolded Chua's circuit . . . . . . . . . . . 783 
2.4. Multiscroll chaotic attractors from the modified Brockett system . . . . . . . . 784

2.5. Circuit realization of $n$-scroll chaotic attractors . . . . . . . . . . . . . . . . . 785

2.5.1. State-controlled CNN realization . . . . . . . . . . . . . . . . . . . . 785

2.5.2. Circuit realization of 3 - and 5 -scroll chaotic attractors . . . . . . . . . . . 786

2.5.3. Realization of $n$-scroll chaotic attractors . . . . . . . . . . . . . . . . . 787

2.5.4. A systematic design approach for generating $n$-scroll chaotic attractors . . 788

2.5.5. An improved design approach for generating $n$-scroll chaotic attractors . . . 791

3. Design of $n$-Scroll Chaotic Attractors via Nonlinear Modulating Functions . . . . . . 793

3.1. Sine function approach . . . . . . . . . . . . . . . . . . . . . . . . . . . . . 793

3.2. Nonlinear transconductor method . . . . . . . . . . . . . . . . . . . . . . . 795

3.3. General nonlinear modulating function approach . . . . . . . . . . . . . . . . 797

3.3.1. Modulating function method . . . . . . . . . . . . . . . . . . . . 798

3.3.2. Adjustable sawtooth wave function approach . . . . . . . . . . . . . . . 798

3.3.3. Adjustable triangular wave function method . . . . . . . . . . . . . . . 799

3.3.4. Adjustable transconductor wave function approach . . . . . . . . . . . . 799

4. Design of Multidirectional Multiscroll Chaotic Attractors via Basic Circuits . . . . . 801

4.1. Step function approach . . . . . . . . . . . . . . . . . . . . . . . . . . . . 801

4.2. Hysteresis series method . . . . . . . . . . . . . . . . . . . . . . . . . 803

4.2.1. Two-dimensional hysteresis system . . . . . . . . . . . . . . . . . . . . 803

4.2.2. Three-dimensional hysteresis system . . . . . . . . . . . . . . . . . . . 805

4.3. Saturated function series approach . . . . . . . . . . . . . . . . . . . . . . . 809

5. Design of Multiscroll Chaotic Attractors via Switching Manifolds . . . . . . . . . . . 814

5.1. Design of chaotic attractors with multiple merged basins of attraction via

switching control . . . . . . . . . . . . . . . . . . . . . . . . 814

5.2. Design of multiscroll chaotic attractors via step series switching . . . . . . . . . 817

6. Design of Multifolded Torus Chaotic Attractors . . . . . . . . . . . . . . . . . . . 819

6.1. A modified multifolded torus chaotic system . . . . . . . . . . . . . . . . . 819

6.2. Circuit implementation . . . . . . . . . . . . . . . . . . . . . . . . . . . . 820

7. Design of Hyperchaotic Multiscroll Attractors . . . . . . . . . . . . . . . . . . . . 822

7.1. Hyperchaotic multiscroll attractors from a simple four-dimensional system . . . . 822

7.2. Hyperchaotic multiscroll attractors from the modified MCK circuit . . . . . . . 823

7.3. Hyperchaotic multiscroll attractors from CNNs . . . . . . . . . . . . . . . . . 827

7.4. Hyperchaotic coupled Chua's circuits . . . . . . . . . . . . . . . . . . . . . . 827

8. Design of Multiscroll Chaotic Attractors via Other Techniques . . . . . . . . . . . . 831

8.1. Multiscroll chaotic attractors from the modified Lorenz system . . . . . . . . . 832

8.2. Multiscroll chaotic attractors from the modified proto-Lorenz system . . . . . . 833

8.3. Multiscroll chaotic attractors from a critical chaotic system . . . . . . . . . . . 834

8.4. Multiscroll chaotic and nonchaotic attractors from the quasi-periodically

forced system . . . . . . . . . . . . . . . . . . . . . . . . . . . 835

8.5. Multiscroll strange attractors in the PWL van der Pol system . . . . . . . . . . 836

8.6. Multiscroll chaotic attractors in the PWL-Duffing system . . . . . . . . . . . . 836

8.7. Multiscroll chaotic attractors in a nonautonomous chaotic system . . . . . . . . 837

8.8. Multiscroll chaotic attractors in a nonautonomous pulse-driven system . . . . . 838

8.9. Multiscroll chaotic attractors in the fractional order systems . . . . . . . . . . 840

9. Implementation and Applications of Multiscroll Chaotic Attractors . . . . . . . . . . 841

9.1. Generating multiscroll chaotic attractors via digital circuit implementation . . . 842

9.2. Digitized $n$-scroll chaotic attractors model for secure communication . . . . . . 844

9.3. Unidirectionally coupled synchronization of multiscroll chaotic attractors . . . . 845 
9.4. Nonlinear $H_{\infty}$ synchronization of $n$-scroll chaotic attractors . . . . . . . . . . . 848

9.5. Controlling $n$-scroll Chua's circuit . . . . . . . . . . . . . . . . . . . . . . . 850

9.6. Recurrent neural state-space model for Chua's double-scroll chaotic attractor . . 851

9.7. True random bit generation via a double-scroll chaotic attractor . . . . . . . . 852

10. Conclusion and Discussion . . . . . . . . . . . . . . . . . . . . . . . . . . . . . 854

\section{Introduction}

Chaos theory has been intensively investigated within the science, mathematics and engineering communities for more than four decades (see, for example [Chen \& Dong, 1998; Chen \& Lü, 2003; Chen \& Yu, 2003; Lü et al., 2002e], and many references cited therein). In particular, Chua's circuit [Chua et al., 1996; Chua, 1998; Kennedy, 1993], as a paradigm of chaos and a bridge between electronic circuits and the chaos theory, has been widely studied and used as a platform for engineering applications. Recently, extended from Chua's circuit, theoretical design and hardware implementation of different kinds of chaotic oscillators have attracted increasing attention, targeting real-world applications of many chaos-based technologies and information systems. It stimulates the current research interest in creating various complex multiscroll chaotic attractors by using simple electronic circuits and devices. After the rapid development in more than a decade, multiscroll chaotic attractors generation has become a relatively mature research direction, and it is time to have an overview on this important subject today.

In retrospect, Suykens and Vandewalle [1991, 1993a, 1993b] first introduced a family of $n$ double scroll chaotic attractors from the so-called quasi-linear function approach. Suykens and Vandewalle [1995] also designed a simple recurrent neural network model that can produce a chaotic attractor like the double-scroll attractor of Chua's circuit. Arena et al. [1995, 1996a, 1996b] realized the generalized $n$-double-scroll Chua's circuit by using a three-state-controlled cellular neural network $(\mathrm{CNN})$ with a generalized piecewise-linear (PWL) output function [Chua, 1998]. Later on, Suykens et al. [1997] proposed a method for generating a more complete family of $n$-scroll instead of $n$-double-scroll chaotic attractors. At about the same time, Suykens and Chua [1997] also investigated the $n$-double-scroll attractors produced by hypercubes in one-dimensional (1D) CNNs. AzizAlaoui $[1999,2000]$ then presented a PWL function approach for creating multispiral chaotic attractors from both autonomous and nonautonomous differential equations. Yalcin et al. [2000a] introduced a technique for generating a family of $n$-double-scroll hyperchaotic attractors. Yalcin et al. [2001] also proposed a simple circuit model for generating $n$-scroll chaotic attractors. The main design idea of most of the aforementioned methodologies is the same - to add some additional breakpoints into the PWL function of the nonlinear resistor in Chua's circuit, or other nonlinear circuits [Yalcin, 2004; Han, 2004].

From a similar but different approach, Tang et al. [2001b] introduced a sine function to generate $n$-scroll chaotic attractors in a simple circuit. Similarly, Özoğuz et al. [2002] suggested a nonlinear transconductor method for creating $n$-scroll chaotic attractors [Salama et al., 2003]. Lü et al. [2002a, 2002f, 2003a, 2003b] presented a switching manifold approach for generating chaotic attractors with multiple-merged basins of attraction. Cafagna and Grassi [2003a, 2003b] developed a coupling Chua's circuit method by using sine nonlinearity instead of PWL nonlinearity for creating one-directional (1-D) $n$-scroll, two-directional (2-D) $n \times m$-grid scroll, and three-directional (3-D) $n \times m \times l$-grid scroll hyperchaotic attractors. Yu et al. [2003a] proposed a family of $n$-scroll hyperchaotic chaotic attractors in a four-dimensional (4D) system, and Yu et al. [2005a, 2005d] introduced a novel nonlinear modulating function approach for generating $n$-scroll chaotic attractors based on a general jerk circuit, which can arbitrarily design the swings, widths, slopes, breakpoints, equilibriums, shapes, and even some general phase portraits. Yu et al. [2004a, 2005c, 2005e] also constructed a family of multifolded torus chaotic attractors, and Chen et al. [2004] developed a switching control technique for generating chaotic torus attractors, which are quite unusual in the chaotic circuit literature. Last but not least, Ahmad [2005] created the $n$-scroll chaotic attractors from the fractional order systems.

Regarding electronic circuits, it is well known that step circuit, hysteresis circuit, and saturated 
circuit are the three types of basic circuits, which have been intensively investigated and applied [Fujiwara et al., 2003, Han et al., 2003a, 2003b, 2005; Nakagawa \& Saito, 1996; Saito, 1990; Saito \& Nakagawa, 1995; Storace \& Parodi, 1998]. Yalcin et al. [2002b] proposed a method using the step circuit to generate a family of scroll-grid chaotic attractors, including 1-D $n$-scroll, 2-D $n \times$ $m$-grid scroll, and 3 -D $n \times m \times l$-grid scroll chaotic attractors. Elwakil and Kennedy [1999, 2001] further investigated some hysteresis-based chaotic oscillators. Lü et al. [2004d] introduced a hysteresis circuit series approach for creating 1-D $n$-scroll, 2-D $n \times m$-grid scroll, and 3-D $n \times m \times l$ grid scroll chaotic attractors. Recently, Lü et al. [2004b, 2004c] also initiated a saturated circuit series approach for generating 1-D $n$-scroll, 2-D $n \times m$-grid scroll, and 3 -D $n \times m \times l$-grid scroll attractors.

Most, if not all, of the aforementioned multiscroll chaotic attractors were verified only by numerical simulations. However, known to electronic engineers, it is much more difficult to physically realize these multiscroll chaotic attractors by analog circuits [Arena et al., 1996a; Yalcin, 2004; Zhong et al., 2002]. But great efforts have been made by many. In this endeavor, Arena et al. [1996a, 1996b] experimentally verified some $n$-double-scroll chaotic attractors by using a state-controlled CNNbased circuit. Yalcin et al. [2000b] experimentally confirmed the 3- and 5-scroll chaotic attractors in a generalized Chua's circuit. Yalcin et al. [1999a] also physically realized a 6 -scroll chaotic attractor in a generalized Chua's circuit using a rescaling breakpoints technique. Elwakil and Kennedy [2000a, 2000b] presented a systematic circuit design method for the realization of a class of hysteresisbased chaotic oscillators [Elwakil, 2000; Elwakil et al., 2000]. Tang et al. [2001b] designed an electronic circuit to experimentally produce $6,7,8$, 9-scroll chaotic attractors in a modified Chua's circuit with the sine nonlinearity instead of the PWL nonlinearity. It is interesting to notice that the largest number of scrolls that could be experimentally confirmed remained 9 until year 2001 . Hardware implementation of $10-$ or more-scroll attractors needs a very large dynamic range in hardware, requiring a very high voltage supply and appropriate differential amplifiers, or a suitable scaling of voltages [Yalcin, 2004], but physical conditions always limit or even prohibit such circuit realizations. Zhong et al. [2002] was the first to experimentally produce and observe a 10scroll chaotic attractor on the oscilloscope, with a systematic chaos generation method introduced. $\mathrm{Yu}$ et al. [2005a, 2005d] then constructed a novel block circuit to realize a maximum of 12 -scroll chaotic attractor in a generalized jerk circuit [Linz \& Sprott, 1999]. More recently, Yu et al. [2005c, 2005e] physically realized a maximum of 9-folded torus chaotic attractor. Finally, it should be mentioned that $\mathrm{Yu}$ et al. [2005b] also experimentally confirmed 3- and 4-scroll hyperchaotic attractors.

Worth mentioning is that it is very difficult to physically realize multiple nonlinear resistors with many segments simultaneously in an electronic circuit. To do so, the electronic device must have a very wide dynamic range, and the corresponding slopes of those segments and their breakpoints must be adjustable, not only easily but also independently toward achieving the simultaneous effect. Therefore, the circuit implementation for generating chaotic attractors of 1 -D $n$-scroll with $n \geq 10,2$-D $n \times m$ grid scroll with $n, m \geq 10$, and 3 -D $n \times m \times l$-grid scroll with $n, m, l \geq 10$, is very difficult technically [Zhong et al., 2002; Lü et al., 2005a, 2005b, 2006]. In the realization of such multidirectional multiscroll chaotic attractors, Yalcin et al. [2002b] were able to experimentally generate and observe a 2 -D $3 \times 3$-grid scroll and a 3 -D $2 \times 2 \times 2$-grid scroll chaotic attractors. Along this line, Lü et al. [2005a, 2006] were able to construct a novel block circuit to experimentally verify a 2 -D $3 \times 11$-grid scroll and a 3 -D $3 \times 3 \times 11$-grid scroll hysteresis chaotic attractors. Very recently, Lü et al. [2005a, 2006] were even able to physically realize 1 -D 14 -scroll, 2 -D $14 \times 10$-grid scroll and 3 -D $10 \times 10 \times 10$-grid (1000) scroll chaotic attractors. It should be pointed out that Lü et al. [2006] was the first to experimentally produce and observe the 2 - $\mathrm{D} n \times m$-grid scroll with $n, m \geq 10$ and 3 -D $n \times m \times l$-grid scroll with $n, m, l \geq 10$ chaotic attractors on the oscilloscope. Moreover, Lü et al. [2005a, 2005b, 2006] also provided a theoretical design principle for hardware implementation of chaotic attractors with multidirectional orientations and with a large number of scrolls simultaneously.

From a theoretical point of view, although all the aforementioned multiscroll chaotic attractors could be confirmed via numerical simulation and/or circuit implementation, it is more desirable to theoretically prove the existence of these visible $n$-scroll chaotic attractors. This, however, is another very difficult task, due to the complex 
dynamical behaviors of the multiscroll chaotic attractors [Silva, 1993]. Nevertheless, Lü et al. [2004d] firstly constructed a two-dimensional (2D) Poincaré return map to rigorously prove the existence of the hysteresis multiscroll chaotic attractors. Similarly, Lü et al. [2004b, 2004c] also theoretically verified the chaotic behaviors of the saturated multiscroll chaotic attractors via another 2D Poincaré return map. Furthermore, this constructive proof approach can be easily extended to the proof of the existence of many other complex multiscroll chaotic attractors [Lü et al., 2004b, 2004c, 2004d].

To this end, it should be pointed out that multiscroll chaotic attractors have many practical applications in, for example, broadband signal generation, CNNs, secure and digital communications, and perhaps efficient liquid mixing, to name a few from among others. To increase the complexity of the chaotic dynamics for higher security [Wada et al., 1999], multiscroll chaotic attractors could be used for communication instead of the general topologically simple chaotic attractors. Suykens and Vandewalle [1998] used the 5-scrolls chaotic attractor in their K. U. Leuven time-series prediction competition. Eguchi et al. [1999] introduced a new digital chaotic circuit for generating multiscroll chaotic attractors, with potential application in digital devices. Yalcin et al. [1999b] experimentally verified a nonlinear $H_{\infty}$ synchronization scheme with 5-scroll chaotic attractors. Tang et al. [2001a] proposed a secure digital communication system that can resist intrusion of eavesdroppers during the transmission of signals and data, for which multiscroll attractors may have better performance. More recently, Yalcin et al. [2002a, 2004a, 2004b] also designed a novel "true random bit generator" based on a double-scroll chaotic attractor.

Given all the above-referred theoretical, numerical and electronic developments in generation of various multiscroll chaotic attractors, it is time to have an overview of the related theories, methodologies and hardware implementation techniques, as a stepping stone in the pursuit of a near mature chaos-based technology that may soon have a stimulating and promising future of practical applications. This article is intended to offer such a review, with the hope that it will at least benefit the new comers in both historical literature search and future research outlook.

The rest of the article is organized as follows. In Sec. 2, several PWL function approaches are introduced for generating $n$-scroll chaotic attractors, along with their circuit realizations. Several nonlinear modulating function methods, including the sine function, nonlinear transconductor, modulating function, adjustable sawtooth wave function, adjustable triangular wave function, and adjustable transconductor wave function, etc., for designing $n$-scroll chaotic attractors generators, are discussed in Sec. 3. In Sec. 4, three types of basic circuits: the step circuit, hysteresis circuit and saturated circuit, are applied to the design of various multidirectional multiscroll chaotic attractors. A couple of switching manifold approaches are then presented for creating multiscroll chaotic attractors in Sec. 5. In Sec. 6, theoretical design and circuit implementation of multifolded torus chaotic attractors are further studied. Some effective methods for generating hyperchaotic multiscroll chaotic attractors are described in Sec. 7, while in Sec. 8 some other techniques are explored for creating various multiscroll chaotic attractors. Topics on potential realworld applications of multiscroll chaotic attractors, as well as their digital implementation, control and synchronization, are briefed in Sec. 9. Finally, conclusions with future research outlooks are given in Sec. 10, putting this important research subject into an engineering perspective.

\section{Design of $n$-Scroll Chaotic Attractors via PWL Functions}

In this section, the multibreakpoint PWL function approach is introduced, which can generate $n$-scroll chaotic attractors from Chua's circuit or other simple nonlinear systems.

\subsection{Design of $n$-double-scroll chaotic attractors using quasi-linear functions}

This subsection briefly describes the quasi-linear function method for creating $n$-double-scroll chaotic attractors.

This method is based on Chua's circuit [Kennedy, 1993; Chua et al., 1986], as shown in Fig. 1, described by

$$
\left\{\begin{array}{l}
\frac{d v_{C_{1}}}{d t}=\frac{1}{R C_{1}}\left(v_{C_{2}}-v_{C_{1}}\right)-\frac{1}{C_{1}} g\left(v_{C_{1}}\right) \\
\frac{d v_{C_{2}}}{d t}=\frac{1}{R C_{2}}\left(v_{C_{1}}-v_{C_{2}}\right)+\frac{1}{C_{2}} i_{L} \\
\frac{d i_{L}}{d t}=-\frac{1}{L} v_{C_{2}},
\end{array}\right.
$$




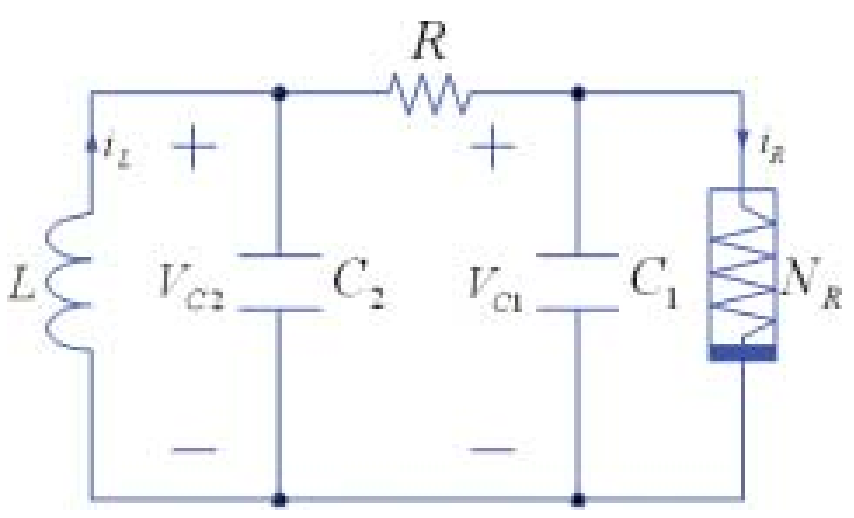

(a) Chua's circuit

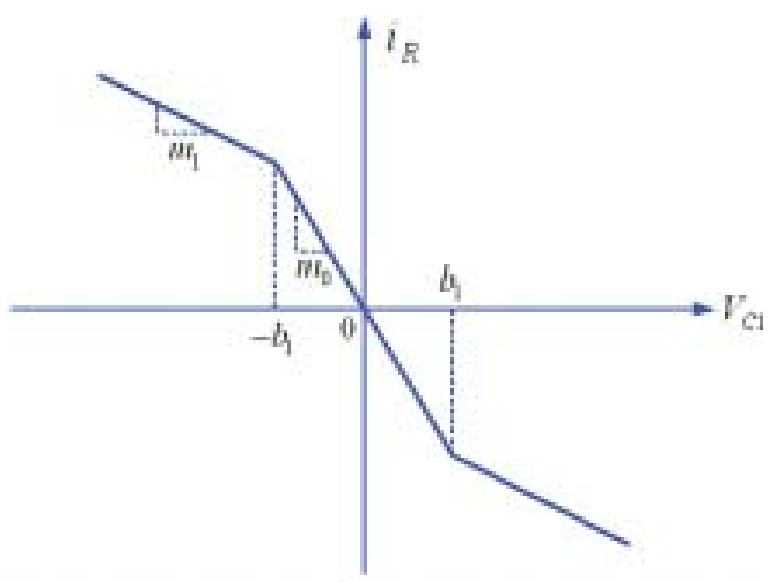

(b) PWL $v-i$ function

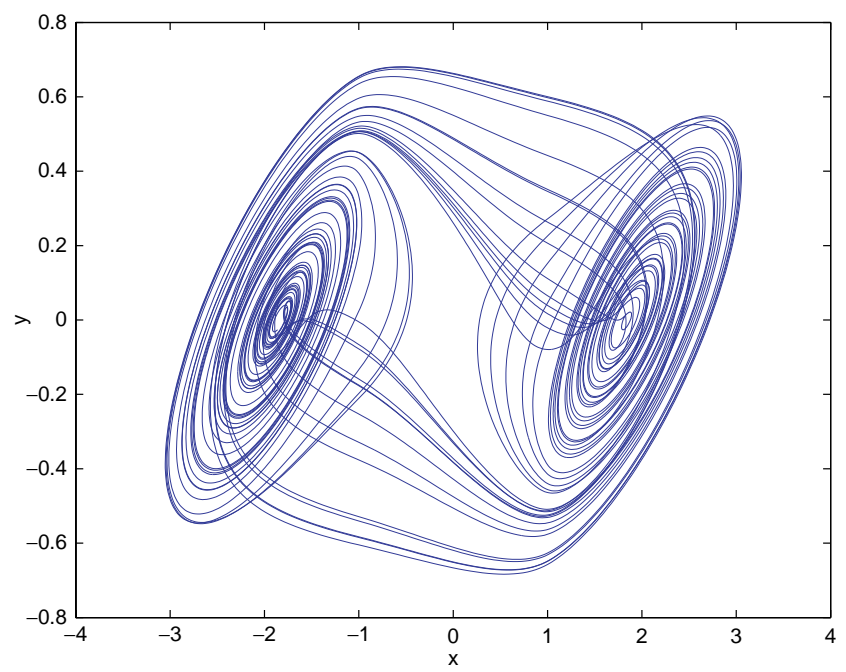

(c) Double-scroll chaotic attractor

Fig. 1. Chua's circuit. where

$$
\begin{aligned}
g\left(v_{C_{1}}\right)= & m_{1} v_{C_{1}}+\frac{1}{2}\left(m_{0}-m_{1}\right) \\
& \times\left(\left|v_{C_{1}}+b_{1}\right|-\left|v_{C_{1}}-b_{1}\right|\right)
\end{aligned}
$$

is a PWL function, as shown in Fig. 1(b).

Let

$$
\begin{aligned}
& x=\frac{v_{C_{1}}}{b_{1}}, \quad y=\frac{v_{C_{2}}}{b_{1}}, \quad z=\frac{R i_{L}}{b_{1}}, \quad \tau=\frac{t}{C_{2} R}, \\
& \alpha=\frac{C_{2}}{C_{1}}, \quad \beta=\frac{C_{2} R^{2}}{L}, \quad \bar{m}_{0}=m_{0} R, \quad \bar{m}_{1}=m_{1} R .
\end{aligned}
$$

Then, system (1) can be written as

$$
\left\{\begin{array}{l}
\dot{x}=\alpha(-x+y-f(x)) \\
\dot{y}=x-y+z \\
\dot{z}=-\beta y
\end{array}\right.
$$

where

$$
f(x)=\bar{m}_{1} x+\frac{1}{2}\left(\bar{m}_{0}-\bar{m}_{1}\right)(|x+1|-|x-1|) .
$$

When $\alpha=10.0, \beta=14.87, \bar{m}_{0}=-0.68, \bar{m}_{1}=$ -1.27 , the system produces a double-scroll chaotic attractor, as shown in Fig. 1(c).

The quasi-linear function approach is a kind of qualitative method, but it gives a global insight into the dynamic behavior of a dynamical system and it can be viewed as a complementary to the conventional linearization approach. Suykens and Vandewalle [1991, 1993a, 1993b] applied the quasilinear function approach to system (1).

First, the system is rewritten in the following form:

$$
\left(\begin{array}{l}
\dot{x} \\
\dot{y} \\
\dot{z}
\end{array}\right)=\left(\begin{array}{ccc}
-a-k(x) & a & 0 \\
b & -b & 1 \\
0 & -c & 0
\end{array}\right)\left(\begin{array}{l}
x \\
y \\
z
\end{array}\right)=A\left(\begin{array}{l}
x \\
y \\
z
\end{array}\right),
$$

where $x=v_{C_{1}}, y=v_{C_{2}}, z=i_{L}, a=1 / R C_{1}, b=$ $1 / R C_{2}, c=1 / L, k(x)=g(x) / C_{1} x$.

Then, the characteristic equation of the matrix $A$,

$$
1+k(x) \frac{\lambda^{2}+b \lambda+c}{\lambda^{3}+(a+b) \lambda^{2}+c \lambda+c a}=0,
$$

leads to a root-locus for the parameter $k(x)$.

To generate a 2-double-scroll chaotic attractor from system (5), Suykens and Vandewalle [1991, 
1993a] modified $k(x)$ as follows:

$$
k_{2}(x)= \begin{cases}-\frac{8}{7} a & 0<x \leq \delta_{1} \\ \frac{2 a\left(x-\delta_{1}\right)}{7\left(\delta_{2}-\delta_{1}\right)}-\frac{8}{7} a & \delta_{1} \leq x \leq \delta_{2} \\ \frac{2 a\left(x-\delta_{2}\right)}{7\left(\delta_{3}-\delta_{2}\right)}-\frac{6}{7} a & \delta_{2} \leq x \leq \delta_{3} \\ -\frac{8}{7} a & \delta_{3} \leq x \leq \delta_{4} \\ \frac{a\left(-0.5 x-0.15\left|x+\delta_{4}\right|+0.15\left|x-\delta_{4}\right|\right)}{0.7 x} & x \geq \delta_{4},\end{cases}
$$

where $a, \delta_{1}, \delta_{2}, \delta_{3}, \delta_{4}$ are design parameters determined in the design procedure.

The nonlinearities $k_{2}(x)$ and $g_{2}(x)$ for creating the 2-double-scroll attractor are shown in the above two sub-figures of Fig. 2 [Suykens \& Vandewalle, 1993a], respectively.

Using the quasi-linear function method to analyze the stability of equilibrium points within different regions, with $\delta_{1}=5, \delta_{2}=7.5, \delta_{3}=10$, $\delta=12, a=7$, system (5) produces a 2 -double-scroll
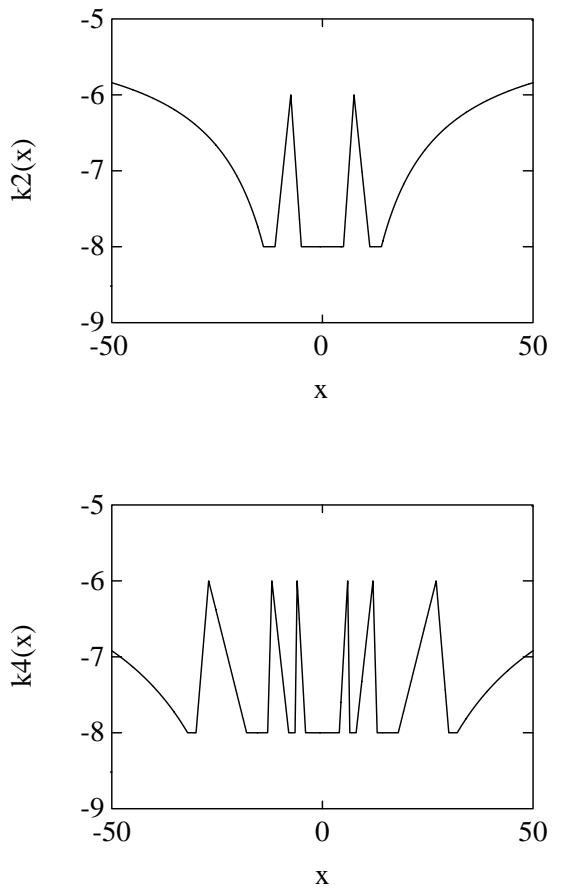

chaotic attractor, as shown in Fig. 3(a) [Suykens \& Vandewalle, 1993a].

To generate $n$-double-scroll $(n \geq 2)$ chaotic attractors, Suykens and Vandewalle [1991, 1993a] further extended system (5) as follows:

$$
\left\{\begin{array}{l}
\dot{x}=\left(-a-k_{n}(x)\right) x+a y \\
\dot{y}=b x-b y+z \\
\dot{z}=-c y
\end{array}\right.
$$
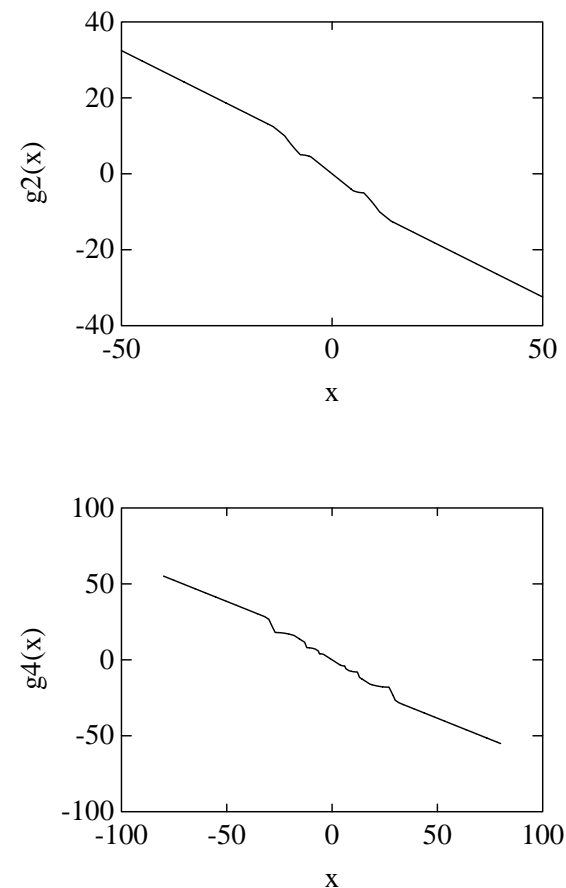

Fig. 2. $k_{n}(x)$ and $g_{n}(x)$ of the $n$-double scroll chaotic attractors $(n=2,4)$ [Suykens \& Vandewalle, 1993a]. 


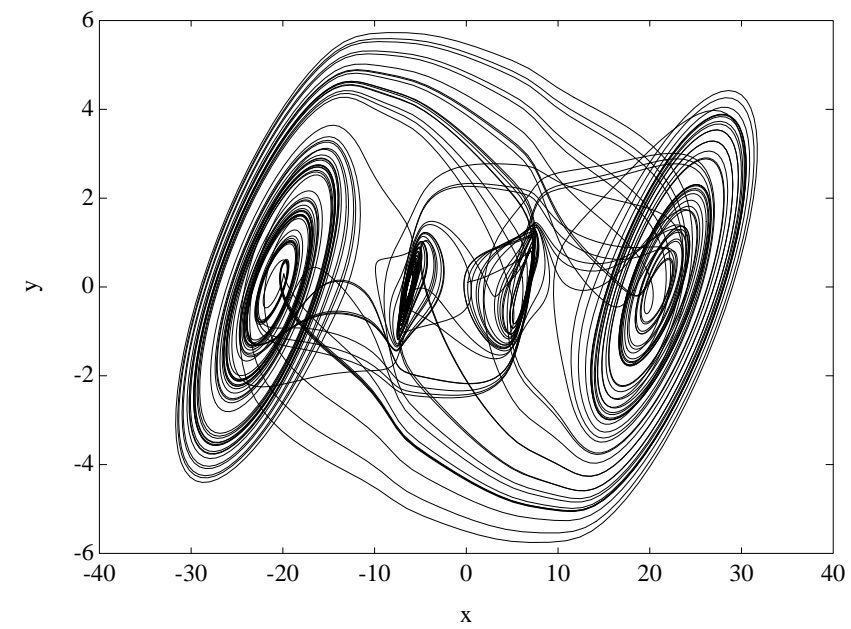

(a) 2-Double-scroll attractor

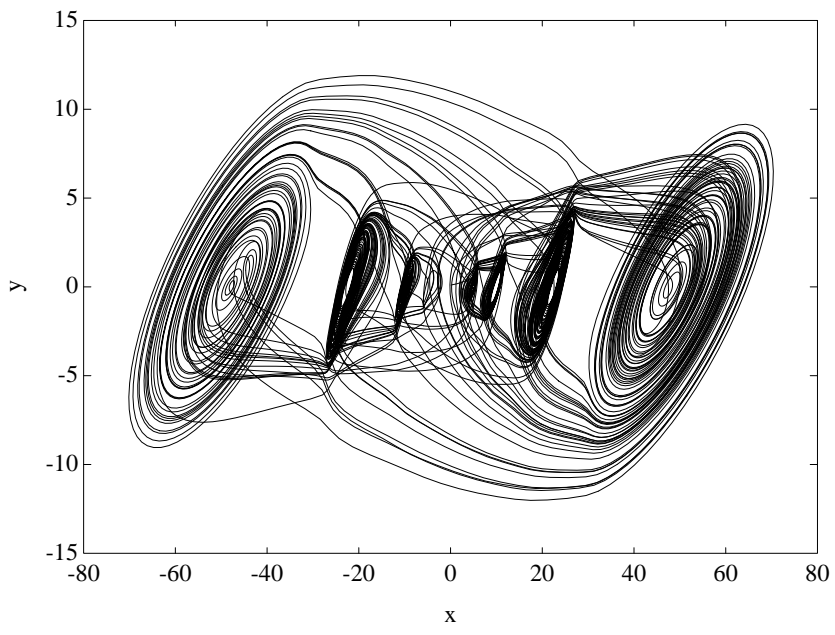

(b) 4-Double-scroll attractor

Fig. 3. $\quad n$-Double-scroll attractors generated via quasi-linear function method [Suykens \& Vandewalle, 1993a].

where

$$
k_{n}(x)= \begin{cases}\alpha_{1} & 0<|x| \leq \delta_{1} \\ \alpha_{2} \frac{|x|-\delta_{1+3(i-2)}}{\delta_{2+3(i-2)}-\delta_{1+3(i-2)}}+\alpha_{1} & \delta_{1+3(i-2)} \leq|x| \leq \delta_{2+3(i-2)} \\ -\alpha_{2} \frac{|x|-\delta_{2+3(i-2)}}{\delta_{3(i-1)}-\delta_{2+3(i-2)}}+\alpha_{1}+\alpha_{2} & 2 \leq i \leq n \\ \alpha_{1} & \delta_{2+3(i-2)} \leq|x| \leq \delta_{3(i-1)} \\ \frac{\alpha_{3}}{|x|}\left(\beta_{1}|x|+\beta_{2}|| x\left|+\delta_{1+3(n-1)}\right|+\beta_{3}|| x\left|-\delta_{1+3(n-1)}\right|\right) & 2 \leq i \leq n \\ & \delta_{3(i-1)} \leq|x| \leq \delta_{1+3(i-1)} \\ & 2 \leq i \leq n\end{cases}
$$

Let $a=7$ and

$$
\frac{\delta_{2+3(i-2)}+\delta_{1+3(i-2)}}{\delta_{2+3(i-2)}-\delta_{1+3(i-2)}}=5 \quad \text { for } 2 \leq i \leq n .
$$

Then, system (7) can generate $n$-double-scroll chaotic attractors. For example, when $\delta_{1}=4, \delta_{2}=$ $6, \delta_{3}=6.5, \delta_{4}=8, \delta_{5}=12, \delta_{6}=13, \delta_{7}=$ $18, \delta_{8}=27, \delta_{9}=30, \delta_{10}=32, a=7$, system (7) has a 4-double-scroll chaotic attractor, as shown in Fig. 3(b) [Suykens \& Vandewalle, 1993a]. The nonlinearities $k_{4}(x)$ and $g_{4}(x)$ for generating the 4-double-scroll attractor are shown in the lower two sub-figures of Fig. 2 [Suykens \& Vandewalle, 1993a], respectively.

Note, however, that since the dimension of those scrolls that are near the origin will become very small, these scrolls are physically invisible for larger $n$.

\section{2. n-Scroll chaotic attractors from a generalized Chua's circuit}

In this subsection, a generalized Chua's circuit is introduced for generating $n$-scroll chaotic attractors.

Suykens et al. [1997] proposed a generalized Chua's circuit, described by

$$
\left\{\begin{array}{l}
\dot{x}=\alpha(y-h(x)) \\
\dot{y}=x-y+z \\
\dot{z}=-\beta y
\end{array}\right.
$$

where $n$ is a natural number, and

$$
\begin{aligned}
h(x)= & m_{2 n-1} x+\frac{1}{2} \sum_{i=1}^{2 n-1} \\
& \times\left(m_{i-1}-m_{i}\right)\left(\left|x+c_{i}\right|-\left|x-c_{i}\right|\right) .
\end{aligned}
$$

System (8) is determined by the parameters set 


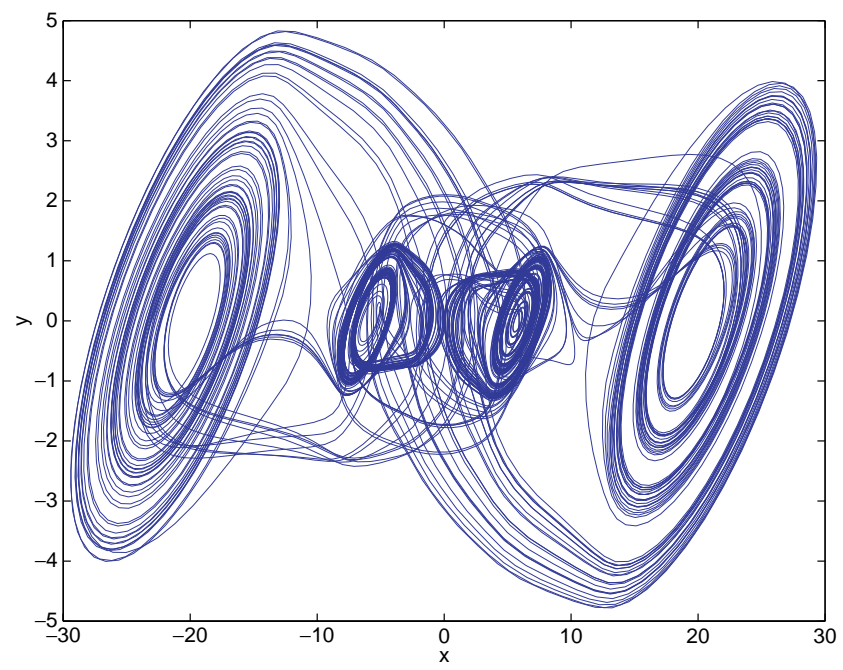

(a) 3-Double-scroll attractor

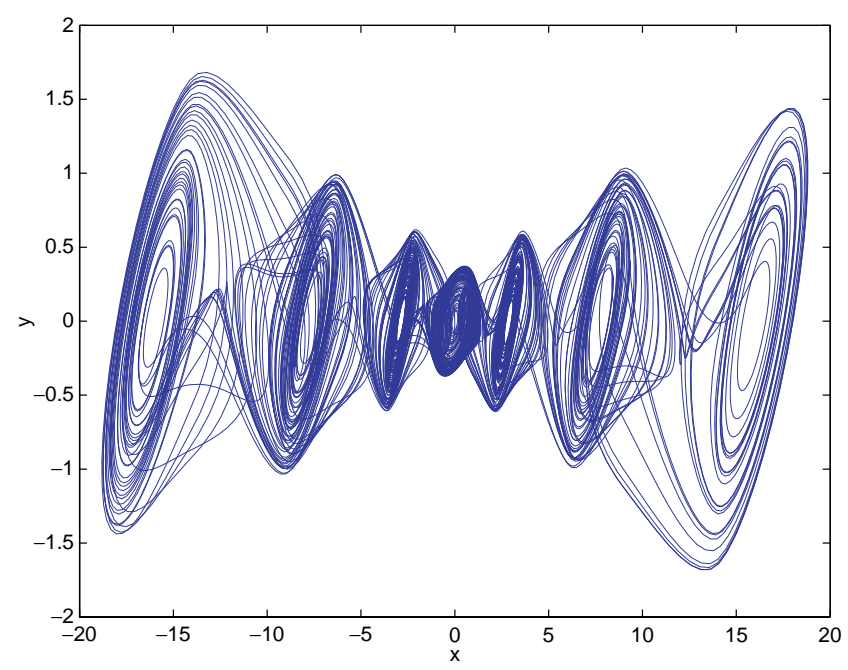

(b) 7-Scroll attractor

Fig. 4. n-Scroll chaotic attractors from a generalized Chua's circuit.

$\{\alpha, \beta, m, c\}$, where $m=\left(\begin{array}{llll}m_{0} & m_{1} & \cdots & m_{2 n-1}\end{array}\right)$ and $c=\left(\begin{array}{llll}c_{1} & c_{2} & \cdots & c_{2 n-1}\end{array}\right)$.

When $\alpha=9, \beta=14.286, m=(-(1 / 7) \quad 2 / 7$ $\left.\begin{array}{llll}-(4 / 7) & 2 / 7 & -(4 / 7) & 2 / 7\end{array}\right), \quad c=\left(\begin{array}{lll}1 & 2.15 & 3.6\end{array}\right.$ $8.213)$, system (8) has a 3 -double-scroll chaotic attractor, as shown in Fig. 4(a). When $\alpha=9$, $\beta=14.286, m=\left(\begin{array}{llll}0.9 / 7 & -3 / 7 & 3.5 / 7 & -(2.4 / 7\end{array}\right)$ $2.52 / 7 \quad-(1.68 / 7) \quad 2.52 / 7 \quad-(1.68 / 7)), c=(1$ $\left.\begin{array}{llllll}2.15 & 3.6 & 6.2 & 9 & 14 & 25\end{array}\right)$, this system has a 7 scroll chaotic attractor, as shown in Fig. 4(b).

Remark 1. In the case of $n$-double-scroll attractors, the components of vector $m$ have alternating signs and the sign of $m_{0}$ is negative. In the case of $n$-scroll attractors with an odd number of scrolls, the components of vector $m$ also have alternating signs, but the sign of $m_{0}$ is positive.

\section{3. $n$-Scroll chaotic attractors from the unfolded Chua's circuit}

This subsection introduces a family of $n$-scroll chaotic attractors generated from the unfolded Chua's circuit.

Figure 5 shows the unfolded Chua's circuit, described by

$$
\left\{\begin{array}{l}
\frac{d v_{1}}{d \tau}=\frac{1}{C_{1}}\left(G\left(v_{2}-v_{1}\right)-f\left(v_{1}\right)\right) \\
\frac{d v_{2}}{d \tau}=\frac{1}{C_{2}}\left(G\left(v_{1}-v_{2}\right)+i_{3}\right) \\
\frac{d i_{3}}{d \tau}=-\frac{1}{L}\left(v_{2}+R_{0} i_{3}\right),
\end{array}\right.
$$

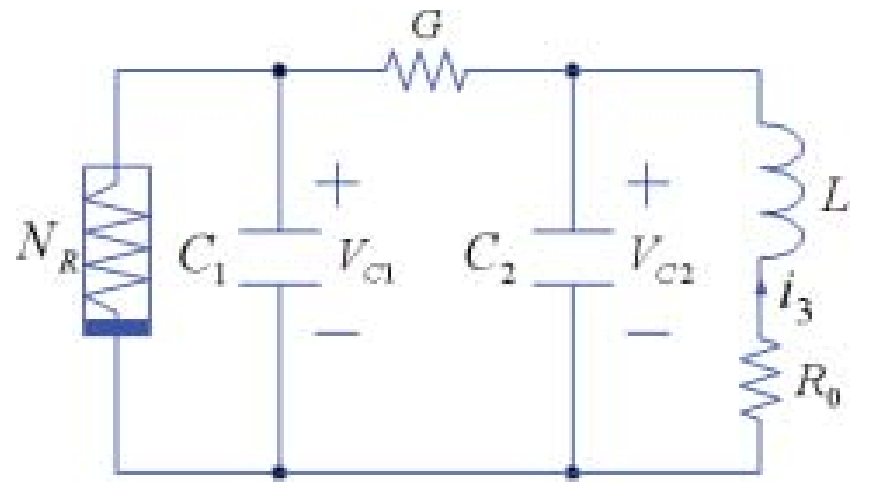

Fig. 5. Unfolded Chua's circuit.

where

$f\left(v_{1}\right)=G_{b} v_{1}+0.5\left(G_{a}-G_{b}\right)\left(\left|v_{1}+E\right|-\left|v_{1}-E\right|\right)$.

Let $x=v_{1} / E, y=v_{2} / E, z=i_{3} / E G, t=$ $\tau G / C_{2}, m_{0}=G_{a} / G, m_{1}=G_{b} / G, \alpha=C_{2} / C_{1}$, $\beta=C_{2} / L G^{2}, \gamma=C_{2} R_{0} / G L$. Then, the corresponding dimensionless state equations are given by

$$
\left\{\begin{array}{l}
\frac{d x}{d t}=\alpha\left(y-x-f_{2}(x)\right) \\
\frac{d y}{d t}=x-y+z \\
\frac{d z}{d t}=-\beta y-\gamma z
\end{array}\right.
$$

where

$f_{2}(x)=m_{1} x+\frac{1}{2}\left(m_{0}-m_{1}\right)[|x+1|-|x-1|]$. 


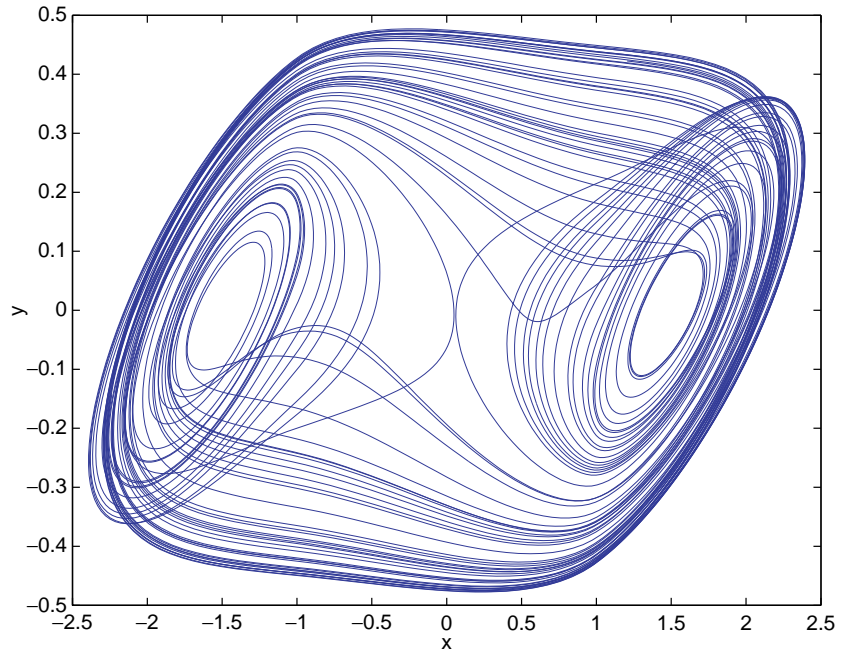

Fig. 6. Double-scroll attractor of the unfolded Chua's circuit.

$$
f_{N}(x)=\left\{\begin{array}{l}
m_{k} x+\operatorname{sgn}(x) \xi_{k} \\
m_{N-1} x+\operatorname{sgn}(x) \xi_{N-1}
\end{array}\right.
$$

in which $N \geq 2, I_{N}=\{0, \ldots, N\}, I_{N}^{*}=\{1, \ldots$, $N\},\left(m_{k}\right)_{k \in I_{N-1}}$, and $\left(\xi_{k}\right)_{k \in I_{N-1}}$ are two finite real sequences, $\left(s_{k}\right)_{k \in I_{N-2}}$ is a finite positive real sequence that is strictly increasing. Moreover, assume that $s_{-1}=0, s_{0}=1, s_{N}=+\infty, \xi_{0}=0$. The parameters $m_{k}$ for $k \in I_{N-1}$ are the slopes of $f_{N}$ in each of the linear segments $\left[-s_{0}, s_{0}\right]$ and $\left[s_{k-1}, s_{k}\right]$ for $k \in I_{N-1}^{*}$.

Remark 2. The function $f_{N}$ is continuous if the parameters $(\xi)_{k \in I_{N-1}}$ satisfy the following condition [Aziz-Alaoui, 1999]:

$$
\begin{aligned}
\xi_{k+1}= & \left(m_{k}-m_{k+1}\right) s_{k}+\xi_{k} \\
& \text { for } \forall \xi_{0} \in \mathbf{R} \text { and } \forall k \in I_{N-2}^{*} .
\end{aligned}
$$

Therefore,

$$
\begin{aligned}
& \xi_{k}=\xi_{0}+\sum_{j=1}^{k}\left(m_{j-1}-m_{j}\right) s_{j-1} \\
& \qquad \text { for } \forall \xi_{0} \in \mathbf{R} \text { and } \forall k \in I_{N-2}^{*} .
\end{aligned}
$$

System (12) is determined by the parameters set $\mathcal{B}_{N}^{C}=\{\alpha, \beta, \gamma\} \cup \mathcal{B}_{N} \subset \mathbf{R}^{2 N+2}$, where $\mathcal{B}_{N}=$ $\left\{\left(s_{k}\right)_{k \in I_{N-2}},\left(m_{k}\right)_{k \in I_{N-1}}\right\}$. To obtain an even number of scrolls, one may use the following parameters set: $\alpha=9.365, \beta=11.79, \gamma=0.04, m_{2 j}=m_{0}=$ $-(8 / 7)$ and $m_{2 j+1}=m_{1}=-(5 / 7)$ for $j=1,2, \ldots$ On the other hand, to obtain an odd number of scrolls, use $\alpha=10.40, \beta=12.5709, \gamma=0.005$,
When $\alpha=9.365, \beta=11.7, \gamma=0.04, m_{0}=-(8 / 7)$, $m_{1}=-(5 / 7)$, system (10) has a chaotic attractor, as shown in Fig. 6.

To create $n$-scroll chaotic attractors, AzizAlaoui [1999] modified the unfolded Chua's circuit (10) as follows:

$$
\left\{\begin{array}{l}
\frac{d x}{d t}=\alpha\left(y-x-f_{N}(x)\right) \\
\frac{d y}{d t}=x-y+z \\
\frac{d z}{d t}=-\beta y-\gamma z
\end{array}\right.
$$

where

$$
\begin{aligned}
& \text { if } s_{k-1} \leq|x| \leq s_{k}, k \in I_{N-2} \\
& \text { if }|x| \geq s_{N-2},
\end{aligned}
$$

$m_{2 j}=m_{0}=-(5 / 7)$ and $m_{2 j+1}=m_{1}=-(8 / 7)$ for $j=1,2, \ldots$.

When $\mathcal{B}_{12}^{C}=\{\alpha=9.365, \beta=11.79, \gamma=0.04$, $s_{0}=1, s_{1}=1.8, s_{2}=2.7, s_{3}=3.36, s_{4}=5.0$, $s_{5}=5.6, s_{6}=6.6, s_{7}=7.15, s_{8}=9.0, s_{9}=9.95$, $\left.s_{10}=10.75\right\}$, system (12) has a 12-scroll chaotic attractor, as shown in Fig. 7(a) [Aziz-Alaoui, 1999]. When $\mathcal{B}_{21}^{C}=\{\alpha=10.40, \beta=12.5709, \gamma=0.005$, $s_{0}=1, s_{1}=4.5, s_{2}=5.8, s_{3}=7.8, s_{4}=9.0$, $s_{5}=11.8, s_{6}=12.9, s_{7}=14.5, s_{8}=15.43$, $s_{9}=17.7, s_{10}=18.7, s_{11}=20.2, s_{12}=21.5$, $s_{13}=23.3, s_{14}=24.2, s_{15}=25.8, s_{16}=26.5$, $\left.s_{17}=28.2, s_{18}=29.12, s_{19}=31.0\right\}$, system (12) has a 21-scroll chaotic attractor, as shown in Fig. 7(b) [Aziz-Alaoui, 1999].

\subsection{Multiscroll chaotic attractors from the modified Brockett system}

In this subsection, a modified Brockett system is introduced, which can generate $n$-scroll chaotic attractors.

The so-called Brockett system is described by [Aziz-Alaoui, 1999, 2000, 2001]

$$
\left\{\begin{array}{l}
\dot{x}=y \\
\dot{y}=z \\
\dot{z}=-\beta y-\gamma z+g(x),
\end{array}\right.
$$




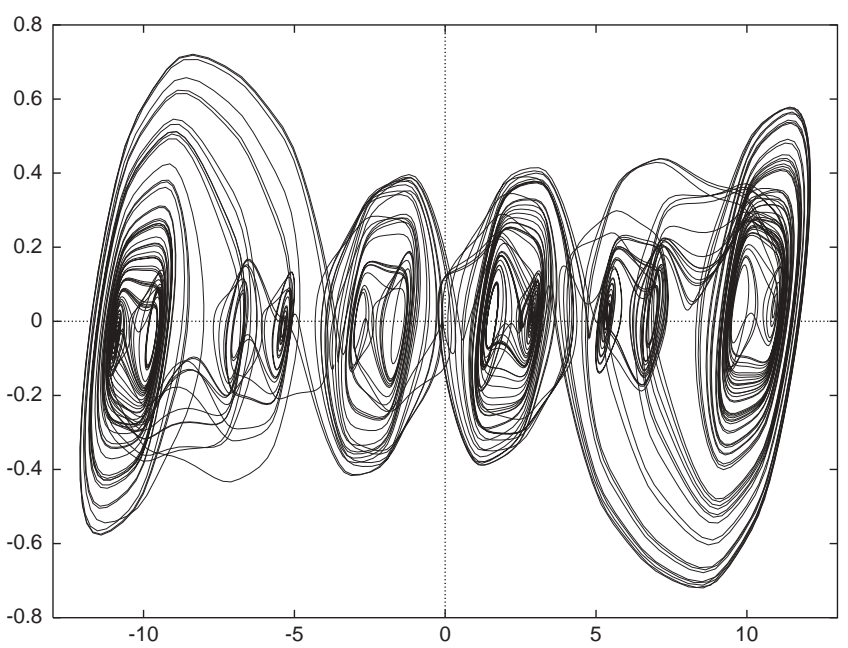

(a) 12-Scroll chaotic attractor

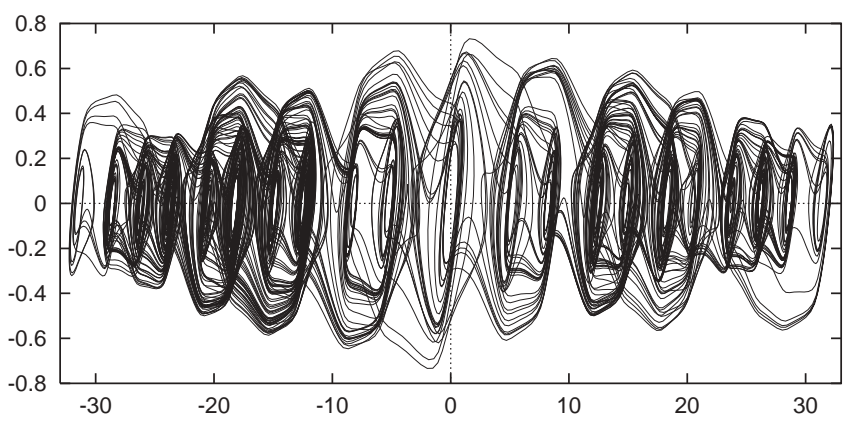

(b) 21-Scroll chaotic attractor

Fig. 7. n-Scroll chaotic attractors from the unfolded Chua's circuit [Aziz-Alaoui, 1999].

where

$$
g(x)= \begin{cases}-K x & \text { if }|x|<1 \\ 2 K x-3 k \operatorname{sgn}(x) & \text { if } 1<|x|<3 \\ 3 K \operatorname{sgn}(x) & \text { if }|x|>3\end{cases}
$$

When $K=-1.8$, system (14) has a double-scroll chaotic attractor.

Since the region $|x|>3$ does not play any role in the dynamics of system (14) [Aziz-Alaoui, 1999], one can replace $g(x)$ by

$$
f_{2}(x)=m_{1} x+\frac{1}{2}\left(m_{0}-m_{1}\right)[|x+1|-|x-1|] .
$$

When $m_{0}=-K=1.8, m_{1}=2 K$, the modified system (14) with (15) shows a similar double-scroll chaotic attractor.

To generate $n$-scroll chaotic attractors, AzizAlaoui [1999] extended system (14) to obtain the following modified Brockett system:

$$
\left\{\begin{array}{l}
\dot{x}=y \\
\dot{y}=z \\
\dot{z}=-\beta y-\gamma z+f_{N}(x)
\end{array}\right.
$$

where $f_{N}(x)$ is given in (13).

Define the following parameters set:

$$
\mathcal{B}_{N}^{B}=\{\beta, \gamma\} \cup \mathcal{B}_{N} \subset \mathbf{R}^{2 N+1},
$$

where $\mathcal{B}_{N}=\left\{\left(s_{k}\right)_{k \in I_{N-2}},\left(m_{k}\right)_{k \in I_{N-1}}\right\} \subset \mathbf{R}^{2 N-1}$, and let $m_{0}=m_{2 i}=-K$ and $m_{1}=m_{2 i+1}=2 K$ for $i=1,2, \ldots$.

Figure 8(a) displays a 4-scroll chaotic attractor [Aziz-Alaoui, 1999], where $s_{0}=1.0, K=-1.99$ and $\mathcal{B}_{4}^{B}=\left\{\beta=1.06, \gamma=0.827, s_{1}=1.65\right.$, $\left.s_{2}=2.1\right\}$. Figure $8(\mathrm{~b})$ shows a 6 -scroll chaotic attractor [Aziz-Alaoui, 1999], where $s_{0}=1.0, K=$ -1.99 and $\mathcal{B}_{6}^{B}=\mathcal{B}_{4}^{B} \cup\left\{s_{3}=2.5, s_{4}=4.1\right\}$.

\subsection{Circuit realization of $n$-scroll chaotic attractors}

In this subsection, some recent advances in circuit realization of $n$-scroll chaotic attractors are reviewed and discussed.

\subsubsection{State-controlled CNN realization}

Arena et al. [1996a, 1996b] experimentally confirmed an $n$-double scroll chaotic attractor by a state-controlled CNN-based circuit.

Consider the generalized Chua's circuit:

$$
\left\{\begin{array}{l}
\frac{d x}{d t}=\alpha(y-h(x)) \\
\frac{d y}{d t}=x-y+z \\
\frac{d z}{d t}=-\beta y-\gamma z
\end{array}\right.
$$

where $h(x)$ is a PWL function defined by

$h(x)=m_{2 n-1} x+\sum_{k=1}^{2 n-1} \frac{m_{k-1}-m_{k}}{2}\left(\left|x+b_{k}\right|-\left|x-b_{k}\right|\right)$,

in which $\alpha, \beta, \gamma$, the $2 n$ coefficients $m_{k}$, and the $2 n-1$ breakpoints $b_{k}$, are all system parameters.

It was proved [Arena et al., 1996a, 1996b] that system (17) was equivalent to a single-layer threecell state-controlled CNN with the following parameters: $C_{1,2}=\alpha, C_{1,1}=1-\alpha m_{2 n-1}, A_{1,1}=1$, 


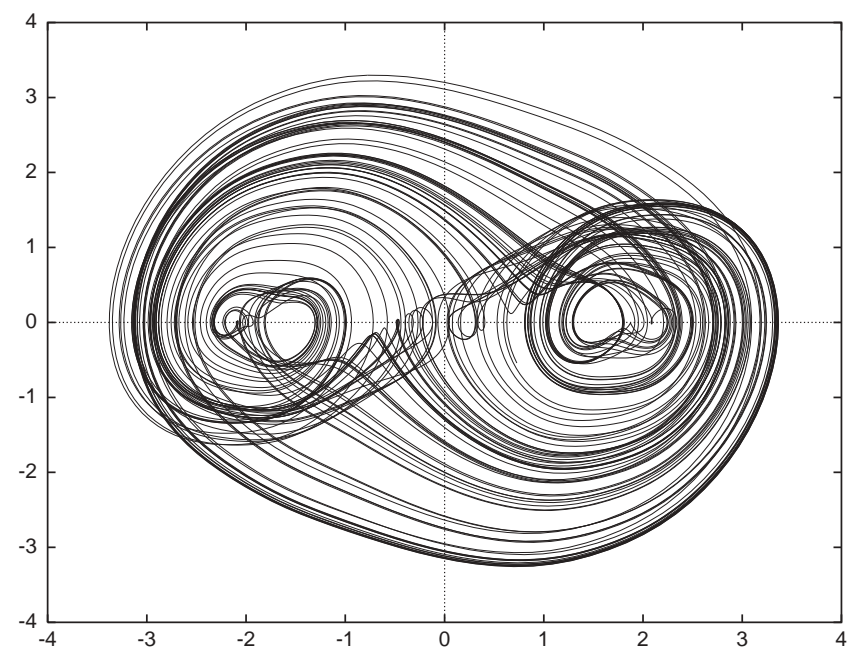

(a) 4-Scroll chaotic attractor

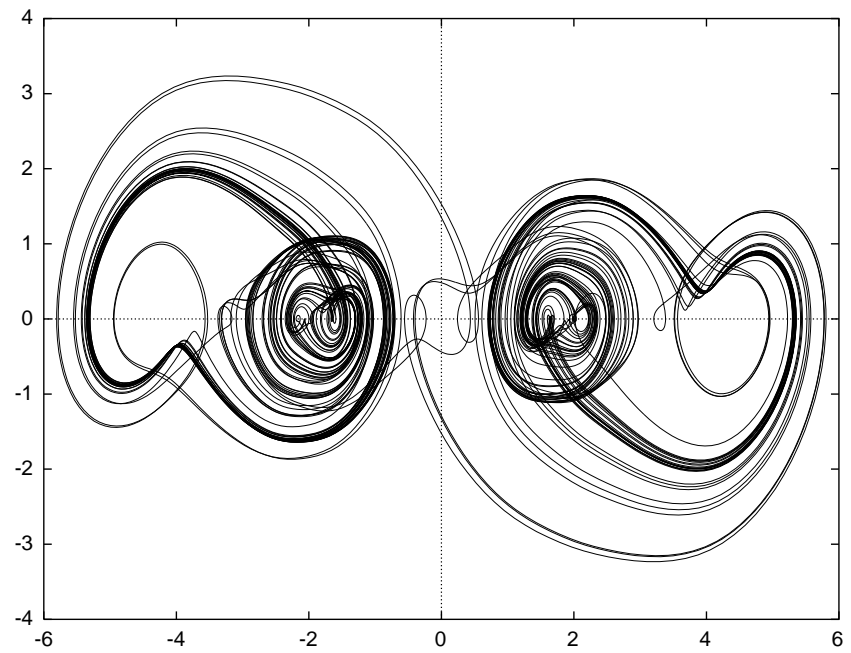

(b) 6-Scroll chaotic attractor

Fig. 8. $n$-Scroll chaotic attractors from the modified Brockett system [Aziz-Alaoui, 1999].

$C_{3,3}=1-\gamma, C_{2,1}=C_{2,3}=1, C_{3,2}=-\beta$, using the following nonlinear output function:

$$
y_{j}=\frac{1}{2} \sum_{k=1}^{2 n-1} n_{k}\left(\left|x_{j}+b_{k}\right|-\left|x_{j}-b_{k}\right|\right),
$$

where $n_{k}=\alpha\left(m_{k}-m_{k-1}\right)$ for $k=1, \ldots, 2 n-1$.

Figure 9 shows a 2-double-scroll chaotic attractor by using the state-controlled CNN realization [Arena et al., 1996a, 1996b].

\subsubsection{Circuit realization of 3 - and 5-scroll chaotic attractors}

Yalcin et al. [2000b] experimentally verified 3- and 5 -scroll chaotic attractors in the generalized Chua's circuit (8).

To begin with, recall the circuit realization of Chua's circuit. Figure 10 shows the voltagecontrolled voltage-source (VCVS) implementation of Chua's circuit. For this type of realization, the corresponding state equations are described by

$$
\left\{\begin{array}{l}
\frac{d x}{d t}=\alpha(-(1+\delta) x+y+f(x)) \\
\frac{d y}{d t}=x-y+z \\
\frac{d z}{d t}=-\beta y
\end{array}\right.
$$

with $f(x)=-h(x)+(1+\delta) x$ and $\delta=1$, where $h(x)$ is defined in (8).

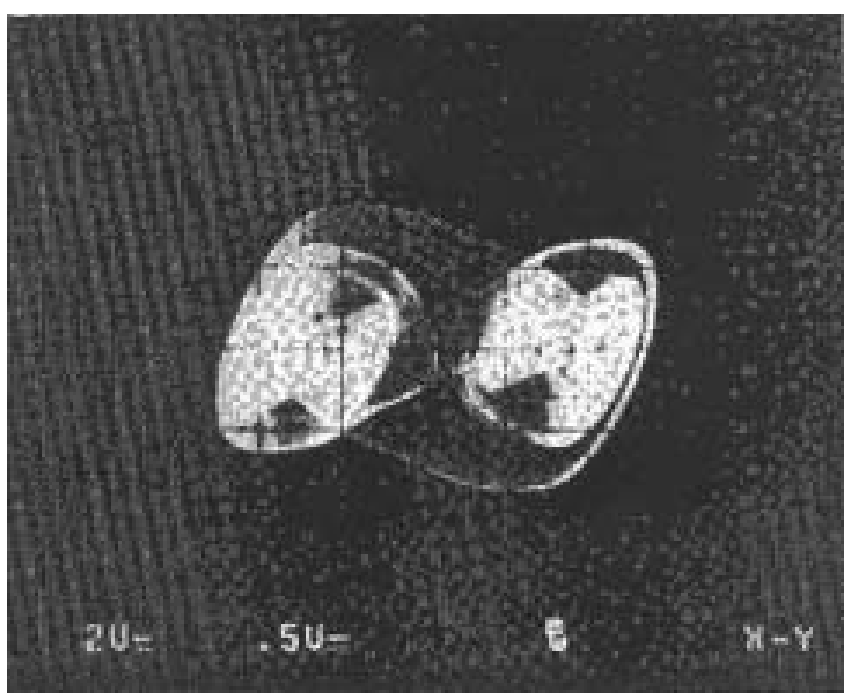

Fig. 9. Observed 2-double-scroll chaotic attractor obtained by using the state-controlled CNN realization ( $h$-axis: $x_{1}$; $v$-axis: $x_{2}$ ) [Arena et al., 1996a, 1996b].

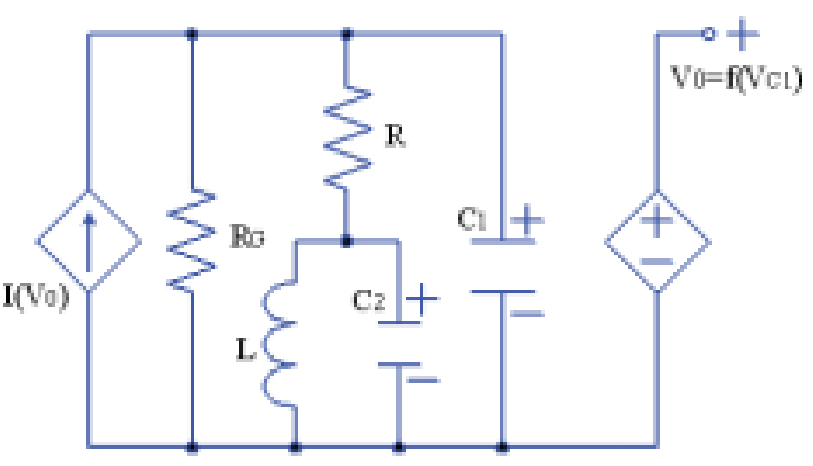

Fig. 10. VCVS implementation of Chua's circuit. 


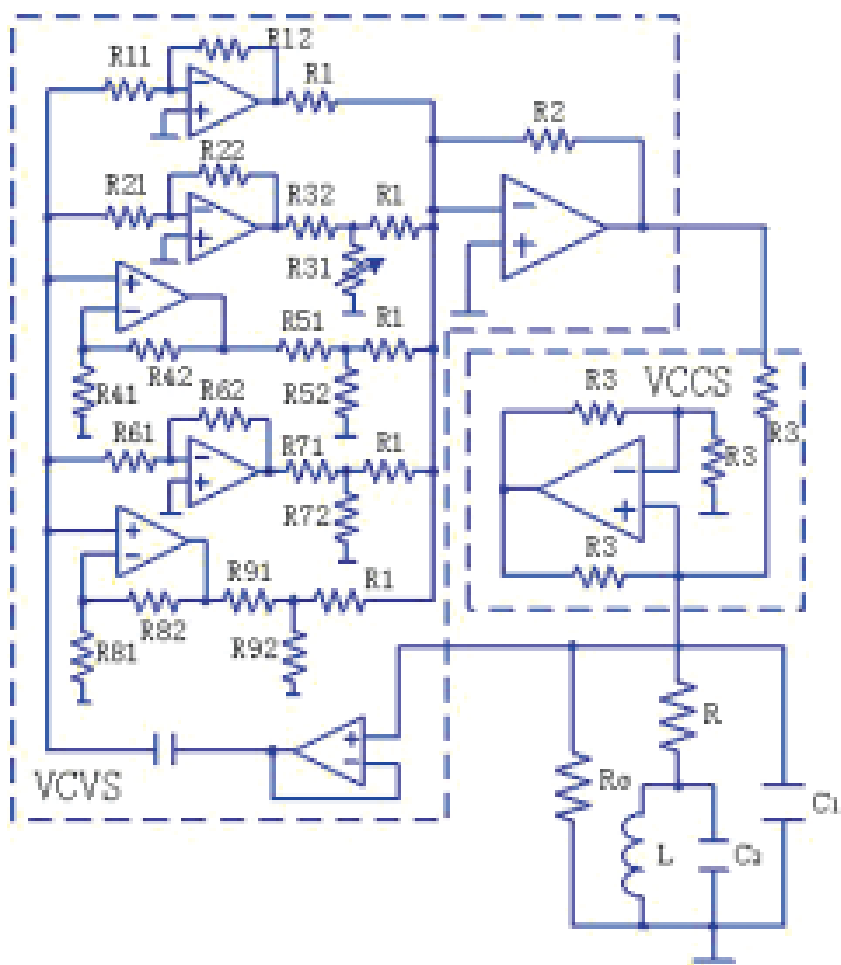

Fig. 11. Circuit implementation of 5-scroll attractor from a generalized Chua's circuit.

Yalcin et al. [2000b] designed the circuit diagram (Fig. 11) for physically realizing a 5-scroll chaotic attractor. The breakpoints are adjusted with the gains of inverting/noninverting amplifiers, as follows:

$$
\begin{aligned}
& c_{1}=\frac{E_{\mathrm{sat}}}{1+\frac{R_{82}}{R_{81}}}, \quad c_{2}=\frac{E_{\mathrm{sat}}}{1+\frac{R_{62}}{R_{61}}}, \\
& c_{3}=\frac{E_{\mathrm{sat}}}{1+\frac{R_{42}}{R_{41}}}, \quad c_{4}=\frac{E_{\mathrm{sat}}}{1+\frac{R_{22}}{R_{21}}} .
\end{aligned}
$$

Moreover, the slopes are also adjusted with the gains of inverting/noninverting amplifiers and voltage branches, as follows:

$$
\begin{aligned}
& m_{4}=-\frac{1}{R_{3}} \frac{R_{2}}{R_{1}}\left(-\frac{R_{12}}{R_{11}}\right) \\
& m_{3}=-\frac{1}{R_{3}} \frac{R_{2}}{R_{1}}\left(m_{4}+\left(-\frac{R_{22}}{R_{21}}\right) \frac{R_{31}}{R_{31}+R_{32}}\right) \\
& m_{2}=-\frac{1}{R_{3}} \frac{R_{2}}{R_{1}}\left(m_{3}+\left(1+\frac{R_{42}}{R_{41}}\right) \frac{R_{52}}{R_{51}+R_{52}}\right) \\
& m_{1}=-\frac{1}{R_{3}} \frac{R_{2}}{R_{1}}\left(m_{2}+\left(-\frac{R_{62}}{R_{61}}\right) \frac{R_{72}}{R_{71}+R_{72}}\right) \\
& m_{0}=-\frac{1}{R_{3}} \frac{R_{2}}{R_{1}}\left(m_{1}+\left(1+\frac{R_{82}}{R_{81}}\right) \frac{R_{92}}{R_{91}+R_{92}}\right) .
\end{aligned}
$$

By adjusting the value of $R_{31}$ in 11 from $12280 \Omega$ to $12740 \Omega$, a 3 -scroll chaotic attractor can be obtained, as shown in Fig. 12(a) [Yalcin et al., 2000b]. When all parameters are set as Table 1 in [Yalcin et al., 2000b], a 5-scroll chaotic attractor can be generated, as shown in Fig. 12(b) [Yalcin et al., 2000b].

\subsubsection{Realization of $n$-scroll chaotic attractors}

By using the scaling properties of the nonlinearity in a generalized Chua's circuit, Yalcin et al. [1999a] experimentally confirmed a 6-scroll chaotic attractor.

According to (18), the equilibrium points of the generalized Chua's circuit satisfy

$$
\left\{\begin{array}{l}
h(x)=0 \\
x=-z \\
y=0
\end{array}\right.
$$

with

$m_{0} c_{1}+\sum_{j=1}^{l-1} m_{j}\left(c_{j+1}-c_{j}\right)+\cdots+m_{l}\left(x_{e q l}-c_{l}\right)=0$,

where $l=1, \ldots, 2 n-1$. The equilibrium points $x_{e q l}$ are shown in Fig. 13. 


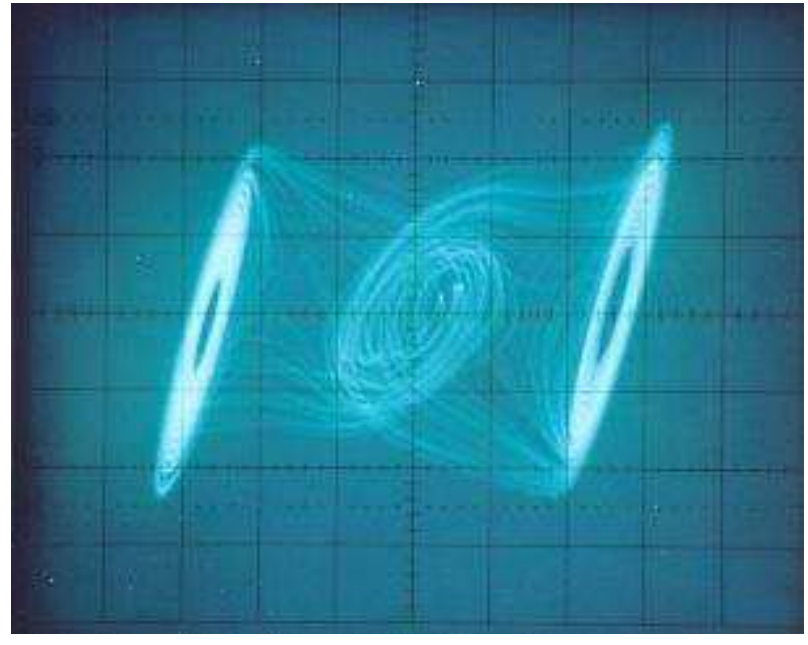

(a) 3-Scroll attractor with $R_{31}=12.280 \Omega$ (h-axis: $0.5 \mathrm{~V} /$ div; $v$-axis: $0.1 \mathrm{~V} /$ div)

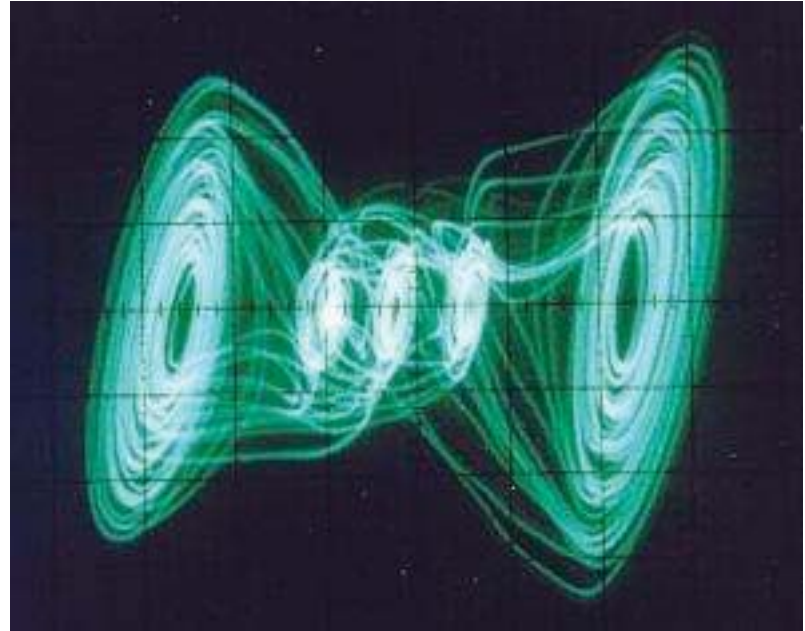

(b) 5-Scroll attractor with $R_{31}=12.740 \Omega$ ( $h$-axis: $1 \mathrm{~V} /$ div; $v$-axis: $0.2 \mathrm{~V} / \mathrm{div}$ )

Fig. 12. Experimental observations of 3- and 5-scroll chaotic attractors [Yalcin et al., 2000b].

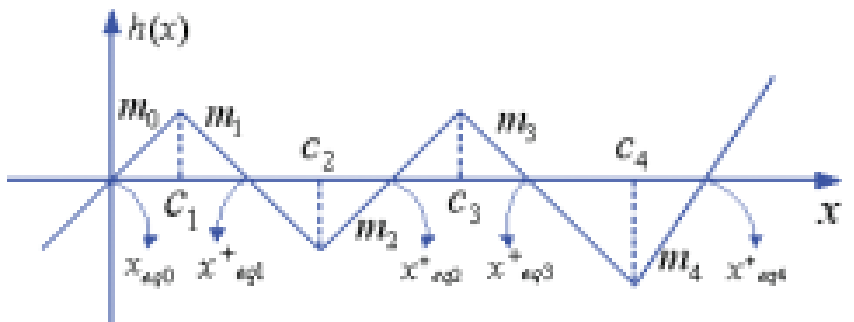

Fig. 13. PWL nonlinearity of the generalized Chua's circuit.

The Jacobi matrix, evaluated at the first equilibrium point $O=(0,0,0)$ and the $i$ th equilibrium point $e q i^{ \pm}=\left(x_{e q i}^{ \pm}, 0, x_{e q i}^{ \pm}\right)$, is respectively

$$
\mathbf{J}(0)=\left(\begin{array}{ccc}
0 & \alpha & 0 \\
1 & -1 & 1 \\
0 & -\beta & 0
\end{array}\right)+\left(\begin{array}{ccc}
-\alpha m_{0} & 0 & 0 \\
0 & 0 & 0 \\
0 & 0 & 0
\end{array}\right)
$$

and

$$
\mathbf{J}\left(e q i^{ \pm}\right)=\left(\begin{array}{ccc}
0 & \alpha & 0 \\
1 & -1 & 1 \\
0 & -\beta & 0
\end{array}\right)+\left(\begin{array}{ccc}
-\alpha m_{i} & 0 & 0 \\
0 & 0 & 0 \\
0 & 0 & 0
\end{array}\right)
$$

where $i=1, \ldots, 2 n-1$.

Yalcin et al. [1999a] rescaled the breakpoints of the PWL function with a factor $k$ in such a way that all the slopes remain the same. After rescaling, the PWL function is

$$
\begin{aligned}
h(x)= & m_{2 n-1} x+\frac{1}{2} \sum_{i=1}^{2 n-1}\left(m_{i-1}-m_{i}\right) \\
& \times\left(\left|x+\frac{c_{i}}{k}\right|-\left|x-\frac{c_{i}}{k}\right|\right) .
\end{aligned}
$$

Then, the new equilibrium points eqi $i^{ \pm}=\left(x_{e q i}^{ \pm} / k\right.$, $\left.0,-x_{\text {eqi }}^{ \pm} / k\right)$ are rescaled with the same factor $k$, as follows:

$$
\begin{aligned}
m_{0} \frac{c_{1}}{k} & +\sum_{j=1}^{l-1} m_{j}\left(\frac{c_{j+1}}{k}-\frac{c_{j}}{k}\right) \\
& +\cdots+m_{l}\left(\frac{x_{e q l}}{k}-\frac{c_{l}}{k}\right)=0 .
\end{aligned}
$$

Figure 14 shows the circuit diagram for a 6scroll chaotic attractor. The values of all components after rescaling current and time by a factor of 10000 are listed in Table 1 in [Yalcin et al., 1999a]. Figure 15 [Yalcin et al., 1999a] displays the observed 6-scroll chaotic attractor.

\subsubsection{A systematic design approach for generating $n$-scroll chaotic attractors}

Until 2000, the largest number of scrolls in a chaotic attractor that could be experimentally verified was only 6. Circuit implementation of more than 6scrolls needs a larger dynamic range of the device, requiring higher voltage supply and appropriate differential amplifiers or a convenient scaling of voltages [Yalcin, 2004]. Lately, Zhong et al. [2002] proposed a new circuit design method for experimentally verifying a maximum of 10 -scroll chaotic attractors.

Consider again Chua's circuit (1). By introducing additional breakpoints into the PWL function (2), the $v-i$ characteristic of the nonlinear resistor 


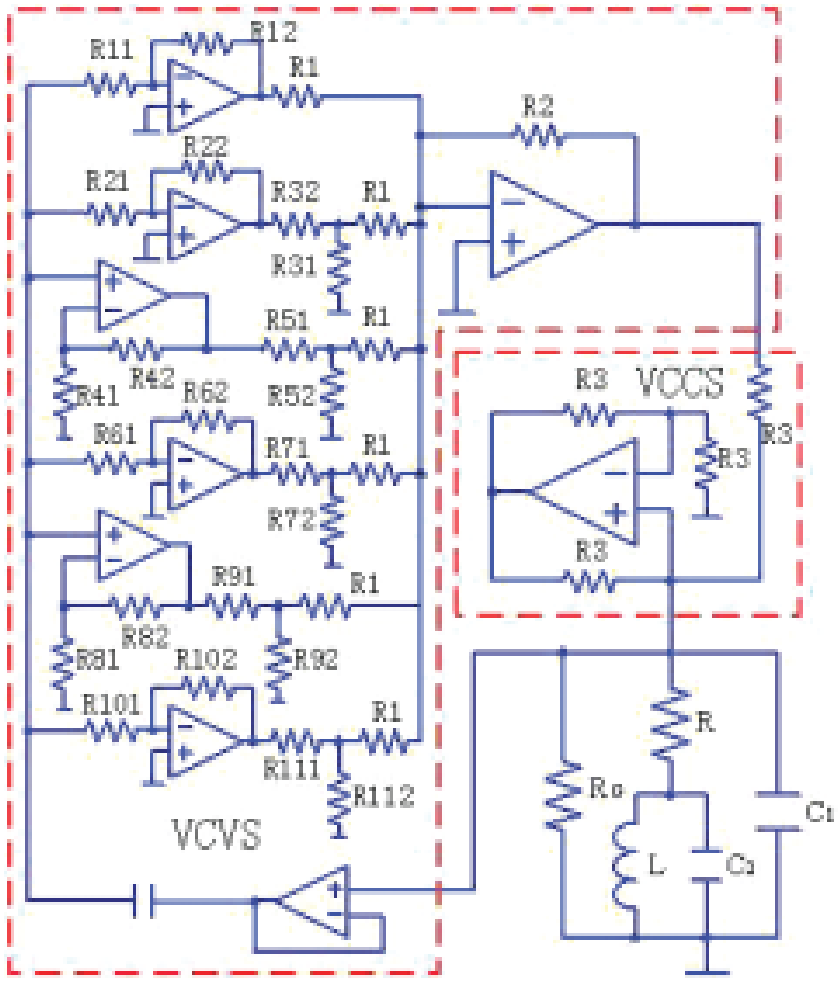

Fig. 14. Circuit diagram for generating a 6-scroll attractor from the generalized Chua's circuit.

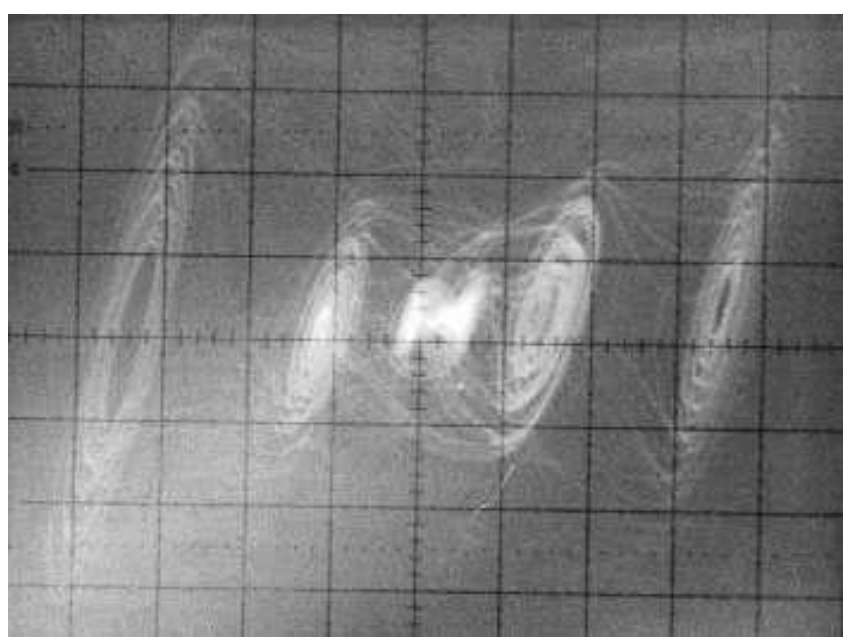

Fig. 15. Observed 6-scroll chaotic attractor $(h$-axis: $1 \mathrm{~V} /$ div; $v$-axis: $0.2 \mathrm{~V} / \mathrm{div}$ ) [Yalcin et al., 1999a].

is modified as follows:

$$
\begin{aligned}
f\left(v_{C_{1}}\right)= & m_{2 n-1} v_{C_{1}}+\frac{1}{2} \sum_{i=1}^{2 n-1}\left(m_{i-1}-m_{i}\right) \\
& \times\left(\left|v_{C_{1}}+b_{i}\right|-\left|v_{C_{1}}-b_{i}\right|\right)
\end{aligned}
$$

where $m_{i}, b_{i}$ are the slopes of the $i$ th segment and the $i$ th breakpoint, respectively. Moreover, attractors with an even number of $2 n$ scrolls can be created by using the nonlinearity (20) embedded in Chua's circuit. Similarly, attractors with an odd number of $2 n-1$ scrolls can be generated by using the following nonlinear resistor:

$$
\begin{aligned}
f\left(v_{C_{1}}\right)= & m_{2 n-1} v_{C_{1}}+\frac{1}{2} \sum_{i=2}^{2 n-1}\left(m_{i-1}-m_{i}\right) \\
& \times\left(\left|v_{C_{1}}+b_{i}\right|-\left|v_{C_{1}}-b_{i}\right|\right) .
\end{aligned}
$$

From Chua's diode shown in Fig. 16, it is clear that there are two basic circuit cells: the left operation amplifier in cell I works in its linear region and the right operation amplifier in cell II works in its whole dynamic range, including the linear and saturated regions. Moreover, the saturated point determines the breakpoint of the PWL function. Therefore, the nonlinear resistors with multisegments can be constructed by using the two cells, I and II, as the basic building blocks of the circuit.

Figure 17 shows the circuit diagram for the nonlinear resistor with 19 segments. It is clear that four pairs of cells biased by VCVS are connected in parallel with Chua's diode. Moreover, two additional breakpoints can be created by connecting one pair of cells II with Chua's diode. Thus, it can implement the nonlinear resistor (20) with $n=5$. Here, each cell is offset by a VCVS for tuning suitable breakpoints. Figure 19(a) displays the measured $v-i$ characteristic with 19 segments.

Figure 18 shows the circuit diagram for the nonlinear resistor with 17 segments, which is realized

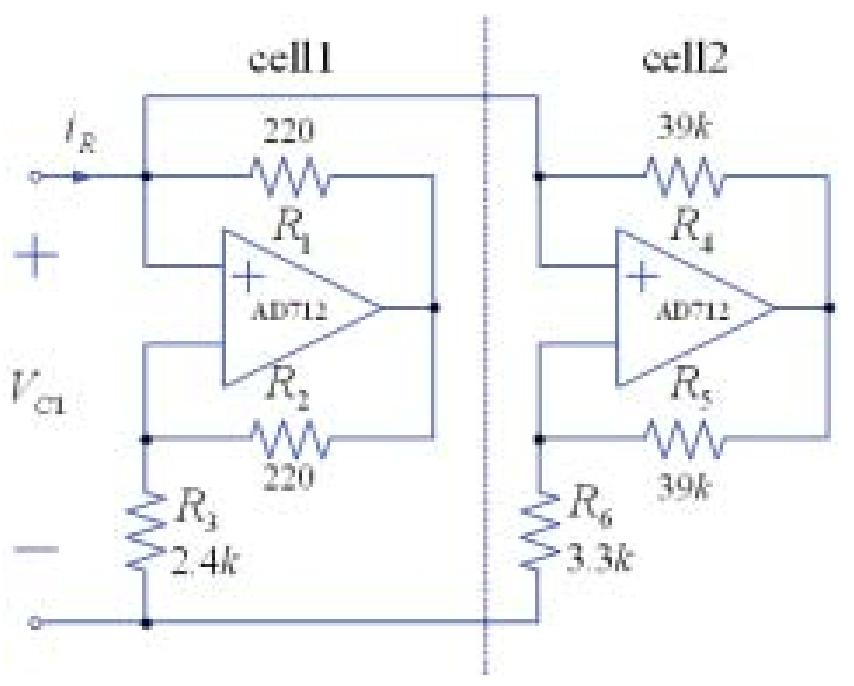

Fig. 16. Chua's diode. 

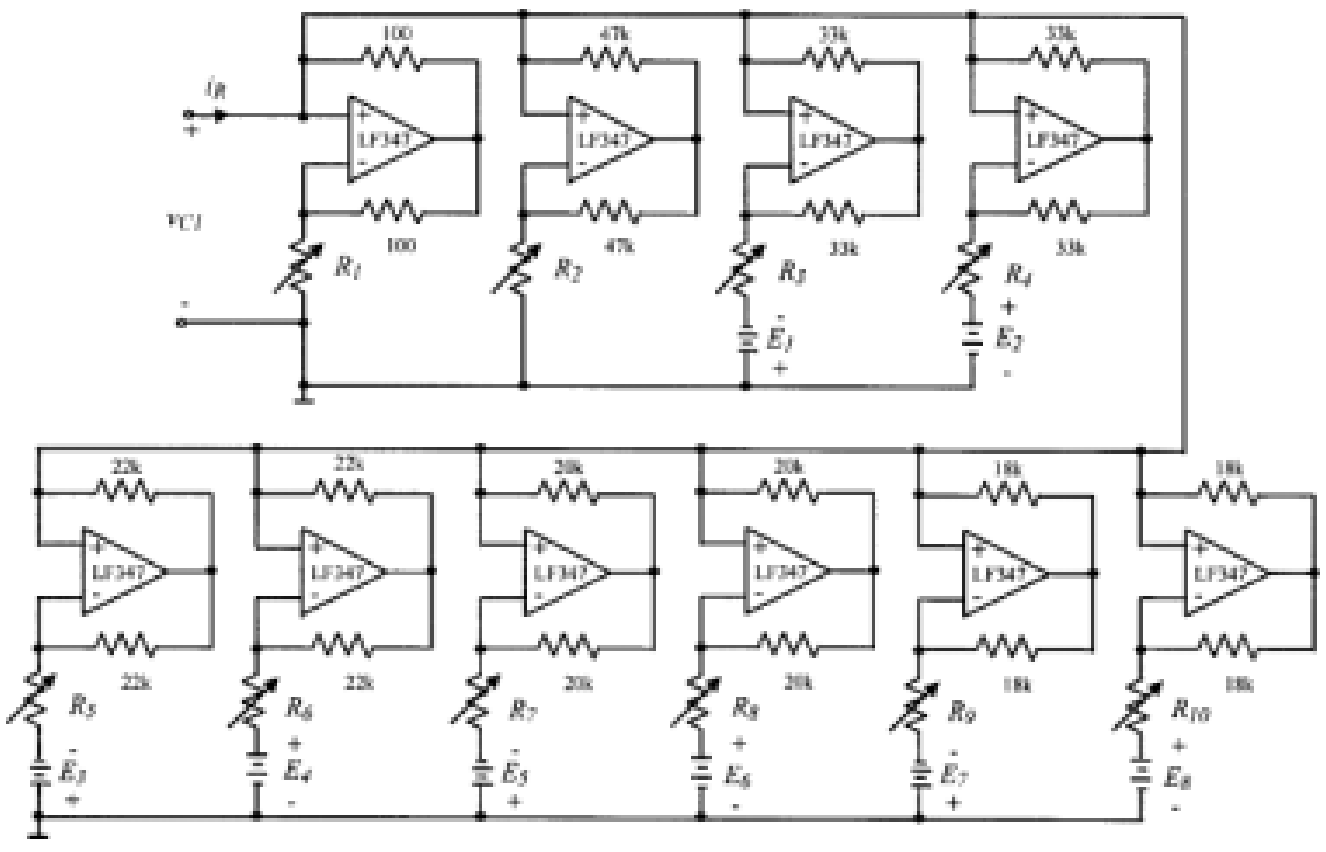

Fig. 17. Circuitry realizing the nonlinear resistor with 19 segments.

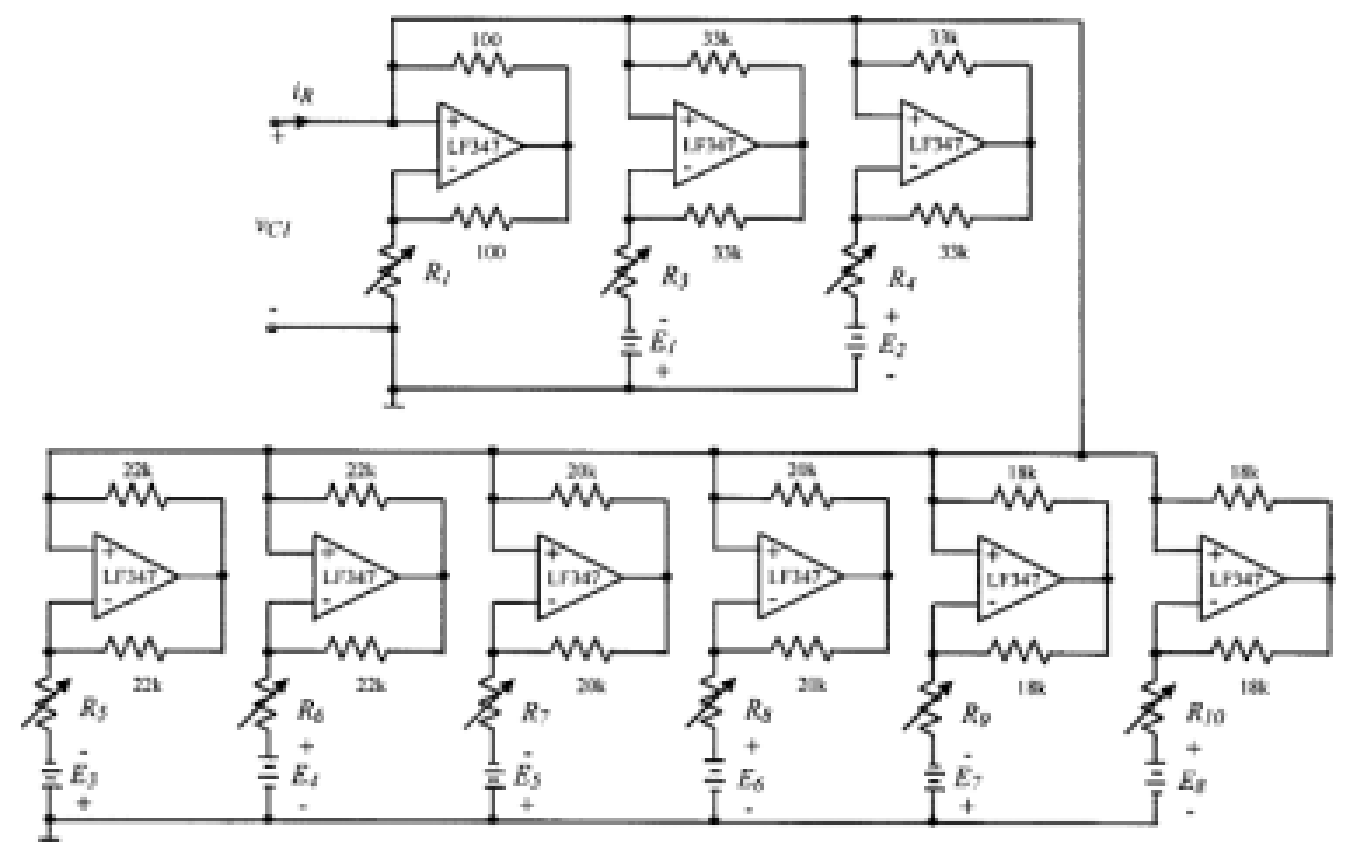

Fig. 18. Circuitry realizing the nonlinear resistor with 17 segments.

by removing the resistor $R_{2}$ from Fig. 17 . Then, it can realize the nonlinear resistor $(21)$ with $n=5$. Figure 19(b) displays the measured $v-i$ characteristic with 17 segments.

Zhong et al. [2002] experimentally verified some chaotic attractors with 5, 6, 7, 8, 9, 10 scrolls, respectively. Figures 20(a) and 20(b) show the experimentally observed 9- and 10-scroll chaotic attractors. All system parameters are listed in Table 1 in [Zhong et al., 2002].

Remark 3. It is quite difficult to create attractors with a large number of scrolls due to the limitation 


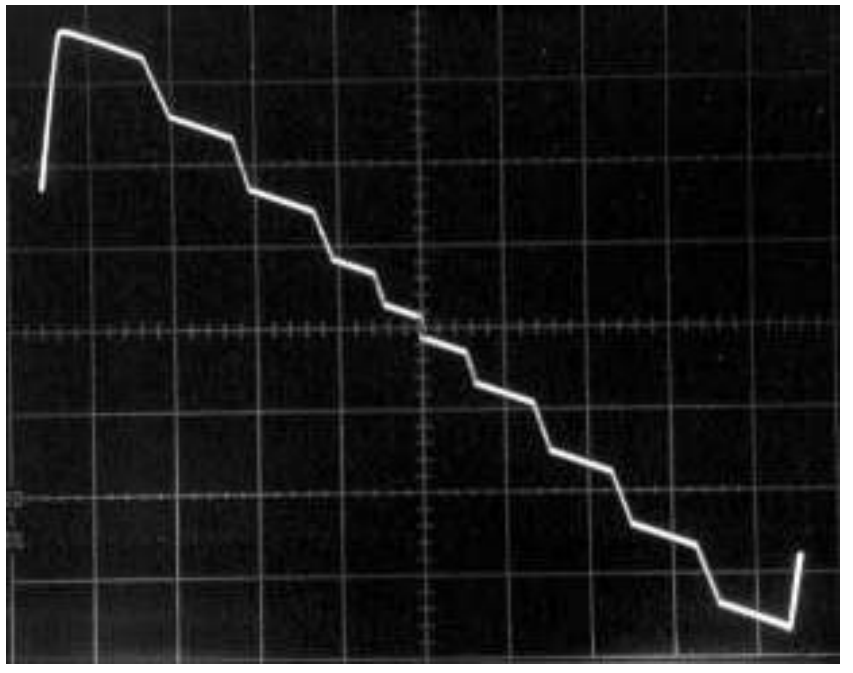

(a) Measured $v-i$ characteristic with 19 segments

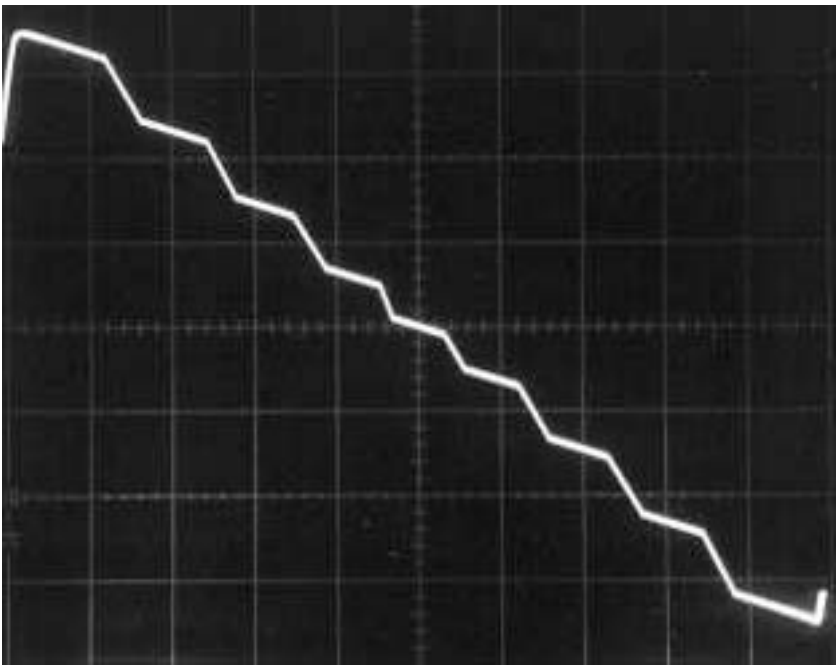

(b) Measured $v-i$ characteristic with 17 segments

Fig. 19. Measured $v-i$ characteristic of the nonlinear resistor.

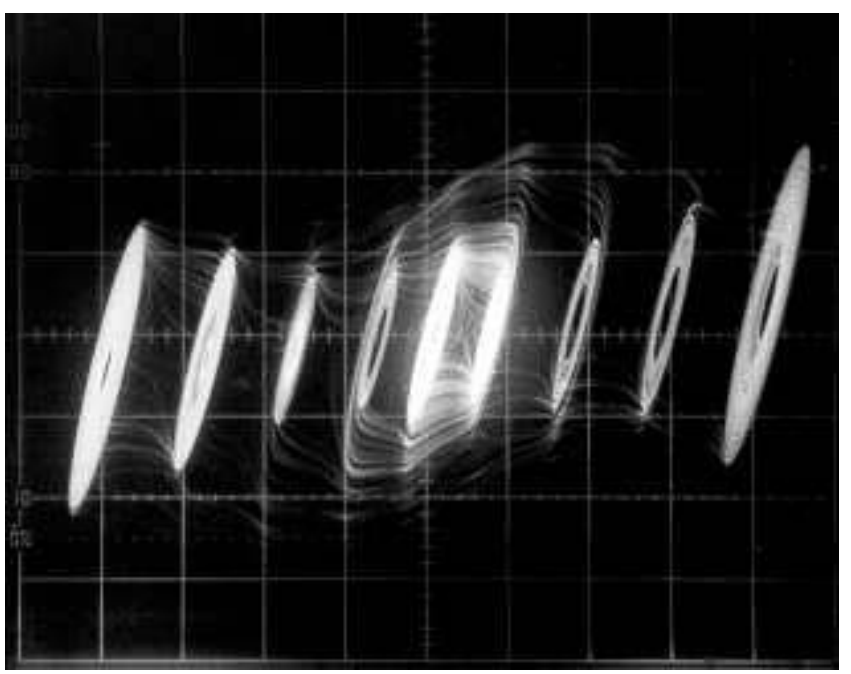

(a) 9-Scroll attractor

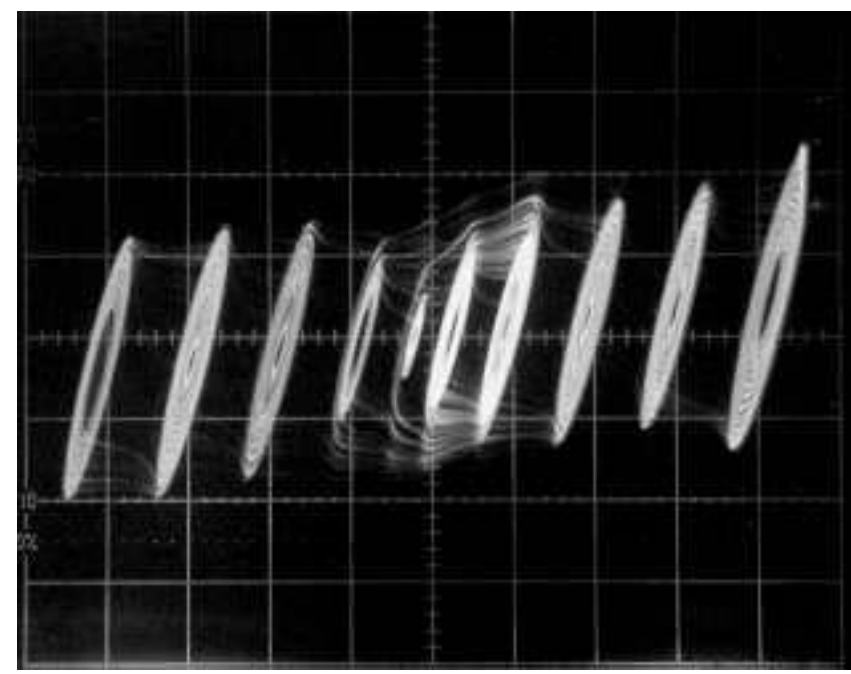

(b) 10-Scroll attractor

Fig. 20. Phase portraits in $v_{C_{1}}-v_{C_{2}}$ plane of $n$-scroll chaotic attractors with horizontal axis $v_{C_{1}}: 2.5 \mathrm{~V} /$ div and vertical axis $v_{C_{2}}: 0.5 \mathrm{~V} /$ div.

of the real dynamic range of the available physical devices.

\subsubsection{An improved design approach for generating $n$-scroll chaotic attractors}

$\mathrm{Yu}$ et al. [2003b] proposed an improved method for generating $n$-scroll chaotic attractors. A general recursive formula was derived for determining the equilibrium points in voltage and breakpoints in voltage. A 11-scroll chaotic attractor was experimentally observed.

Consider Chua's circuit (1), as shown in Fig. 1(a). When $L=9.3 \mathrm{mH}, C_{1}=5.06 \mathrm{nF}, C_{2}=$ $47.9 \mathrm{nF}, G=1 / R=0.6061 \mathrm{mS}, m_{0}=-0.852 \mathrm{mS}$, $m_{1}=-0.32 \mathrm{mS}, b_{1}=0.2 \mathrm{~V}$, system (1) has a double-scroll chaotic attractor. To generate multiscroll chaotic attractors from (1), modify the PWL function (2), as follows: 


$$
f\left(v_{C_{1}}\right)= \begin{cases}m_{0} v_{C_{1}} & 0 \leq\left|v_{C_{1}}\right| \leq b_{1} \\ m_{1} v_{C_{1}}+\sum_{j=1}^{i}\left(m_{j-1}-m_{j}\right) b_{j} & b_{i} \leq\left|v_{C_{1}}\right| \leq b_{i+1}, 1 \leq i \leq n-1 \\ m_{n} v_{C_{1}}+\sum_{j=1}^{n}\left(m_{j-1}-m_{j}\right) b_{j} & \left|v_{C_{1}}\right| \geq b_{n} .\end{cases}
$$

To create some uniform scrolls, assume that $m_{0}=m_{2}=\cdots$ and $m_{1}=m_{3}=\cdots$. If the breakpoints $b_{i}$ are the midpoints of various corresponding PWL segments, then

$$
b_{i+1}=\frac{2 C_{1} \sum_{j=1}^{i}\left(m_{j}-m_{j-1}\right) b_{j}}{G+m_{i}}-b_{i},
$$

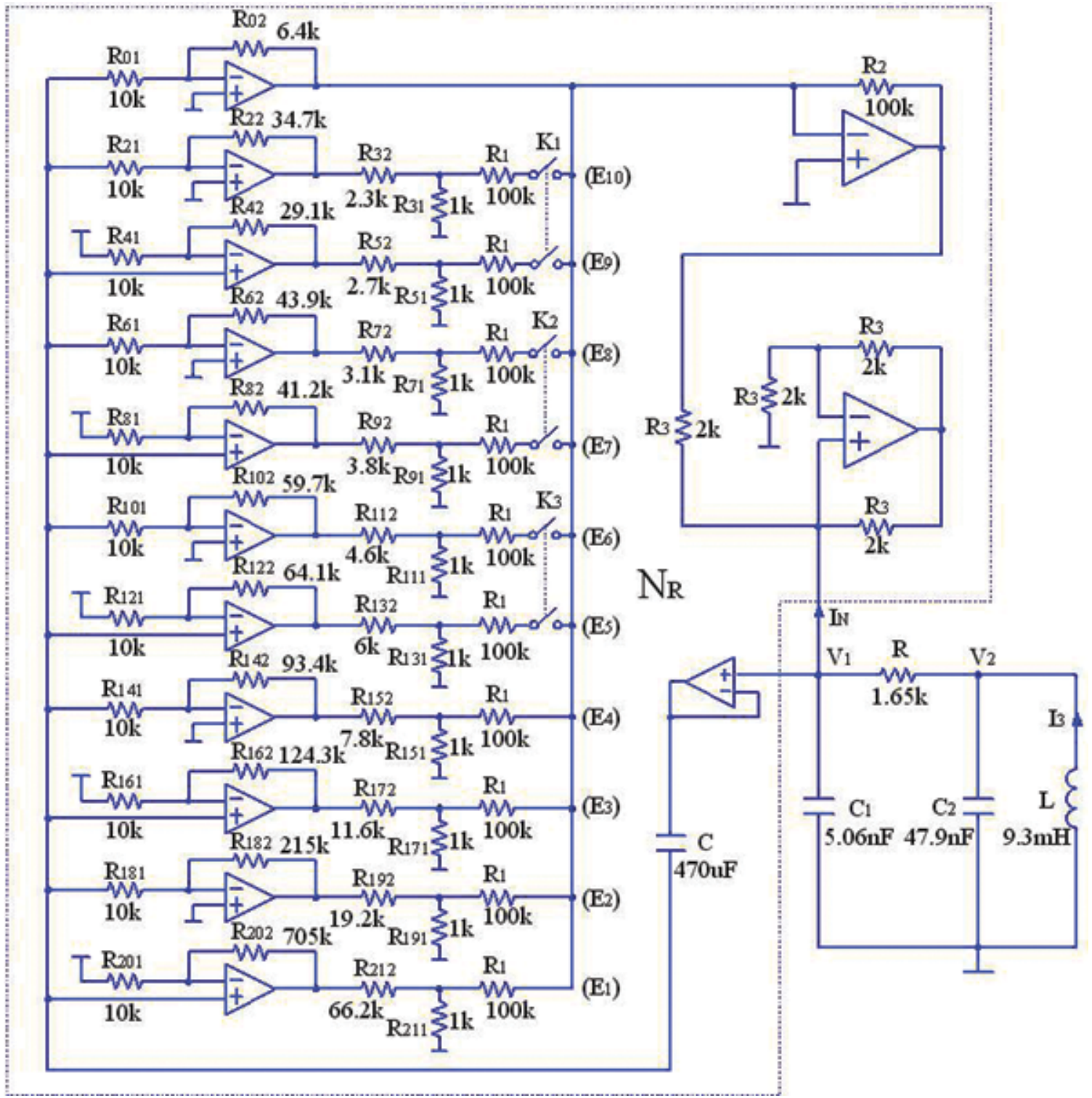

Fig. 21. Circuit diagram for realizing $5 \sim 11$-scroll chaotic attractors [Yu et al., 2003b]. 
where $1 \leq i \leq n-1$. Given parameters $m_{0}, m_{1}, b_{1}$, one can derive all breakpoints $b_{i}$.

If $n$ is an even number, assume that $m_{0}=$ $m_{2}=\cdots=m_{n}=-0.32 \mathrm{mS}$ and $m_{1}=m_{3}=$ $\cdots=m_{n-1}=-0.852 \mathrm{mS}$. Then, system (1) with (22) can generate chaotic attractors with an odd number $(n+1)$ of scrolls. If $n$ is an odd number, assume that $m_{0}=m_{2}=\cdots=m_{n}=$ $-0.852 \mathrm{mS}$ and $m_{1}=m_{3}=\cdots=m_{n-1}=$ $-0.32 \mathrm{mS}$. Then, system (1) with (22) can generate chaotic attractors with an even number $(n+1)$ of scrolls.

Figure 21 [Yu et al., 2003b] displays the circuit diagram for implementing $5 \sim 11$-scroll chaotic attractors. The block within the dotted line plays the same role as the function with Chua's diode $N_{R}$, as shown in Fig. 1(b). All operation amplifiers are $T L 082$. Note that every middle operational amplifier and its additional resistors contribute to a branch circuit of $N_{R}$ and generate a breakpoint $b_{i}$. All circuit parameters can be easily deduced, as given by (12) and (16) in [Yu et al., 2003b]. When switches $K_{1}, K_{2}, K_{3}$ are turned off, Fig. 21 shows the generated 5 -scroll chaotic attractor; when $K_{1}, K_{2}$ are turned off and $K_{3}$ is turned on, it can create a 7 -scroll chaotic attractor; when $K_{1}$ is turned off and $K_{2}, K_{3}$ are turned on, it can generate a 9-scroll chaotic attractor, as shown in Fig. 22(a) [Yu et al., 2003b]; when $K_{1}, K_{2}, K_{3}$ are turned on, it can create a 11-scroll chaotic attractor, as shown in Fig. 22(b) [Yu et al., 2003b].

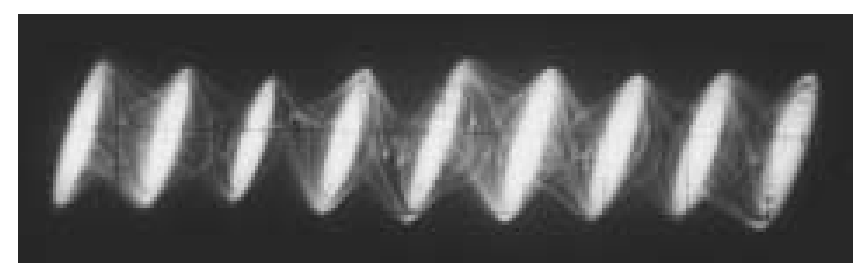

(a) 9-Scroll attractor ( $h$-axis: $0.8 \mathrm{~V} / \operatorname{div}$; $v$-axis: $1 \mathrm{~V} /$ div)

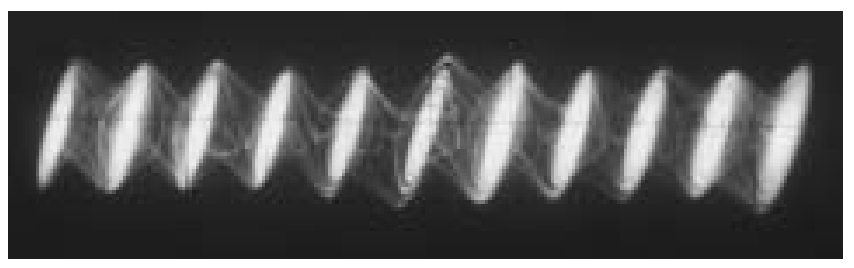

(b) 11 -Scroll attractor ( $h$-axis: $0.95 \mathrm{~V} / \mathrm{div}$; $v$-axis: $1 \mathrm{~V} / \mathrm{div})$

Fig. 22. Experimental observations of 9- and 11-scroll chaotic attractors [Yu et al., 2003b].

\section{Design of $n$-Scroll Chaotic Attractors via Nonlinear Modulating Functions}

In the above section, the PWL function approach was discussed, which can be used for generating multiscroll chaotic attractors. The main idea there was introducing additional breakpoints in the PWL function. In this section, the nonlinear modulating function method is introduced for creating $n$-scroll chaotic attractors.

\subsection{Sine function approach}

Tang et al. [2001b] applied the sine function to replace the nonlinear characteristic function of Chua's circuit and obtained a modified Chua's circuit as follows:

$$
\left\{\begin{array}{l}
\dot{x}=\alpha(y-f(x)) \\
\dot{y}=x-y+z \\
\dot{z}=-\beta y
\end{array}\right.
$$

where

$$
f(x)= \begin{cases}\frac{b \pi}{2 a}(x-2 a c) & x \geq 2 a c \\ -b \sin \left(\frac{\pi x}{2 a}+d\right) & -2 a c<x<2 a c \\ \frac{b \pi}{2 a}(x+2 a c) & x \leq-2 a c,\end{cases}
$$

and

$$
d= \begin{cases}\pi & \text { for } c \text { is even } \\ 0 & \text { for } c \text { is odd }\end{cases}
$$

in which $\alpha, \beta, a, b, c, d$ are real parameters.

Note that system $(23)$ can generate $(c+1)$ scroll chaotic attractors. When $\alpha=10.814, \beta=$ $14.0, a=1.3, b=0.11$, system (23) can create 8 -scroll and 9-scroll chaotic attractors with $c=7$ and $c=8$, as shown in Figs. 23(a) and 23(b), respectively.

Tang et al. [2001b] also constructed an electronic circuit to experimental verify these $n$-scroll chaotic attractors. Figure 24 shows the circuit 


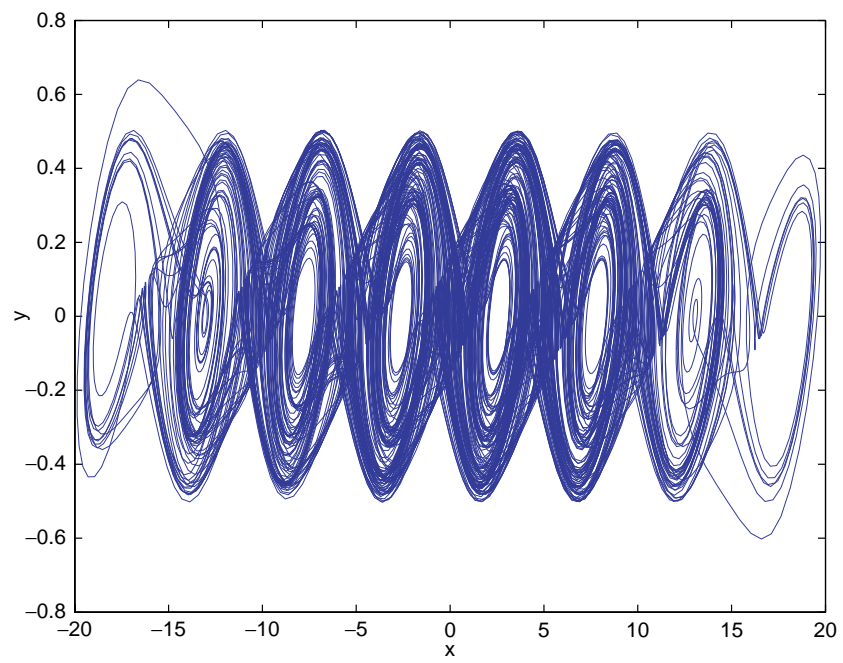

(a) 8-Scroll chaotic attractor

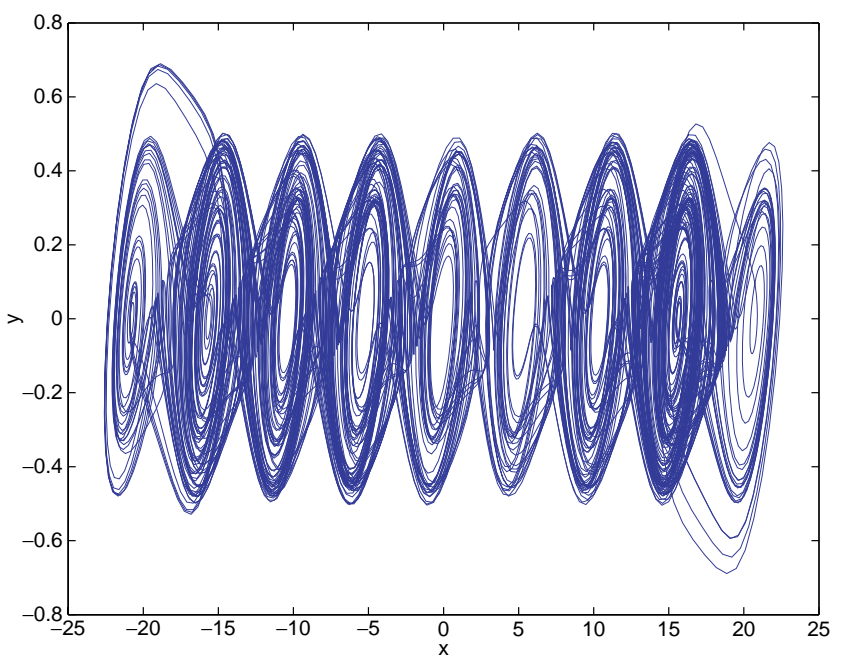

(b) 9-Scroll chaotic attractor

Fig. 23. $n$-Scroll chaotic attractors generated by the sine function method.

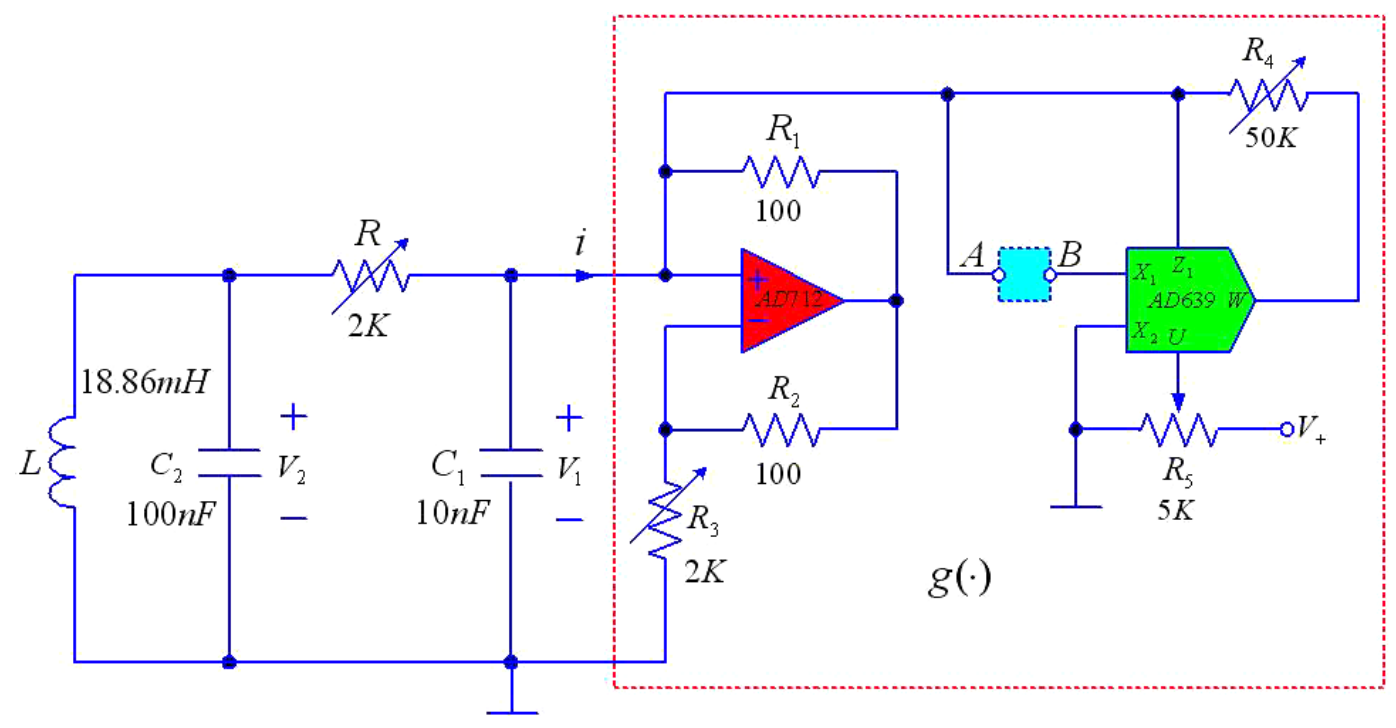

(a) Modified Chua's circuit with $g(x)$

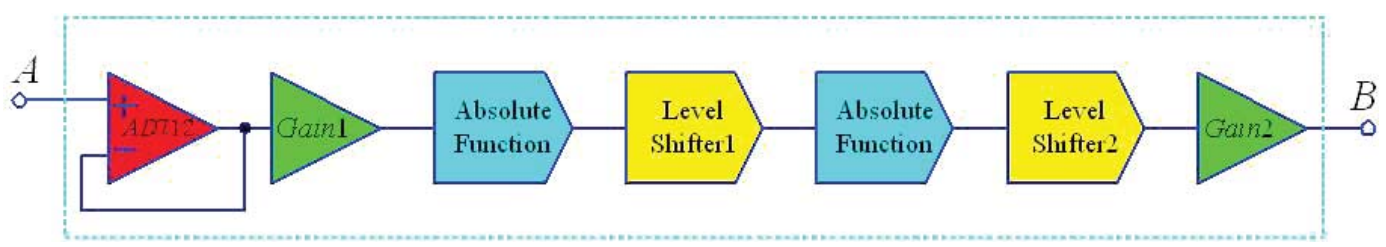

(b) Sine or cosine function generator inserted in $A-B$

Fig. 24. Circuit diagram for generating $n$-scroll chaotic attractors. 
diagram with dynamical equations

$$
\left\{\begin{array}{l}
\frac{d v_{1}}{d t}=\frac{1}{R C_{1}}\left(v_{2}-v_{1}\right)-\frac{g\left(v_{1}\right)}{C_{1}} \\
\frac{d v_{2}}{d t}=\frac{1}{R C_{2}}\left(v_{1}-v_{2}\right)+\frac{i_{L}}{C_{2}} \\
\frac{d i_{L}}{d t}=-\frac{v_{2}}{L},
\end{array}\right.
$$

where $g\left(v_{1}\right)=((b \pi / 2 a)-1 / R) v_{1}-(b \pi / 4 a)\left(\mid v_{1}+\right.$ $\left.2 a c|-| v_{1}-2 a c \mid\right)-b \sin \left(\left(\pi v_{1} / 2 a\right)+d\right)$.

According to Fig. 24(a), the negative resistor $g(\cdot)$ consists of two parts connected in parallel: a one-port described by a $v-i$ characteristic with a linear negative slope, and a one-port with a $v-i$ characteristic described by a sine function. A commercial trigonometric function chip AD639 was used for the circuit design. The original angular input range of $A D 639$ was $\pm 500^{\circ}$ generating a maximum of 4-scroll attractor.

To increase the range of the angular input, for $x \in[-(2 m+1) \pi,(2 m+1) \pi]$, one has

$$
\cos (x)=\cos (|x|)=\sin \left(m \pi+\frac{\pi}{2}-|x|\right)=\sin (y),
$$

where $y=m \pi+(\pi / 2)-|x| \in[-(2+(1 / 2)) \pi,(m+$ $(1 / 2)) \pi]$. It means that the number of scrolls generated by $A D 369$ can be doubled by using an absolute operation and a voltage shift. Figure 24(b) is used for generating larger number of scrolls, inserted into $A-B$ in Fig. 24(a). Figure 25 displays the detailed circuit design for the gain, level shifter, and absolute operation. Figure 26 shows the experimental observations of $6, \sim 9$-scroll chaotic attractors.

\subsection{Nonlinear transconductor method}

In this subsection, the nonlinear transconductor approach [Özoğuz et al., 2002; Salama et al., 2003] is introduced for creating $n$-scroll chaotic attractors.
Özoğuz et al. [2002] introduced an $n$-scroll chaotic attractors generator, whose nonlinearity was the smooth hyperbolic tangent functions [Salama et al., 2003], which is described by

$$
\left(\begin{array}{c}
\dot{x} \\
\dot{y} \\
\dot{z}
\end{array}\right)=\left(\begin{array}{ccc}
0 & 1 & 0 \\
0 & 0 & 1 \\
0 & -a & -a
\end{array}\right)\left(\begin{array}{l}
x \\
y \\
z
\end{array}\right)+\left(\begin{array}{c}
0 \\
0 \\
-a f\left(x_{1}\right)
\end{array}\right),
$$

where

$$
f(x)=\sum_{j=-N}^{M}(-1)^{j-1} \tanh k\left(x-o_{j}\right)
$$

and $M, N$ are odd integers. System (25) can generate a $(M+N+2) / 2$-scroll chaotic attractor.

When $a=0.25, M=5, N=3, k=2$ and $o_{j}=2 j$ for $j[-N, M]$, system (25) has 5-scroll chaotic attractors, as shown in Fig. 27(a).

Figure 28(a) [Özoğuz et al., 2002; Salama et al., 2003] displays the circuit diagram for generating $n$-scroll chaotic attractors. It includes a unity gain voltage buffer, a single current-feedback operational amplifier (CFOA), and a transconductor $g\left(V_{C_{1}}\right)$. Figure 28(b) [Özoğuz et al., 2002; Salama et al., 2003] shows the transconductor, which is realized by using alternating bipolar D-P cells.

It is noticed that the smooth hyperbolic tangent function $f(x)$ in (26) can be replaced by the switching sign function, as follows [Salama et al., 2003]:

$$
f(x)=\sum_{j=-N}^{M} \operatorname{sgn} k\left(x-o_{j}\right) .
$$

When $a=0.25, M=5, N=3, k=2$ and $o_{j}=2 j$ for $j[-N, M]$, system (25) with (27) has a 5-scroll chaotic attractor, as shown in Fig. 27(b). To realize this square-wave-shaped nonlinearity, the

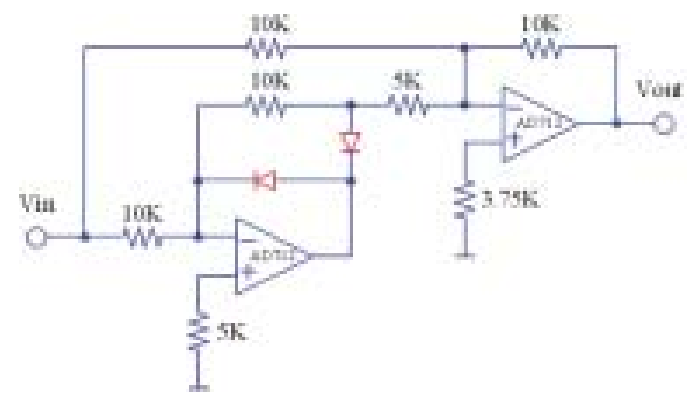

(a) Absolute operation

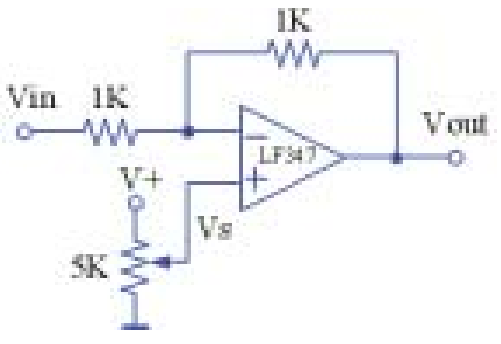

(b) Level shifter

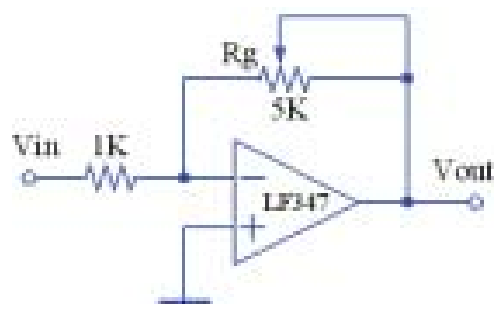

(c) Gain

Fig. 25. Subcircuit diagram. 


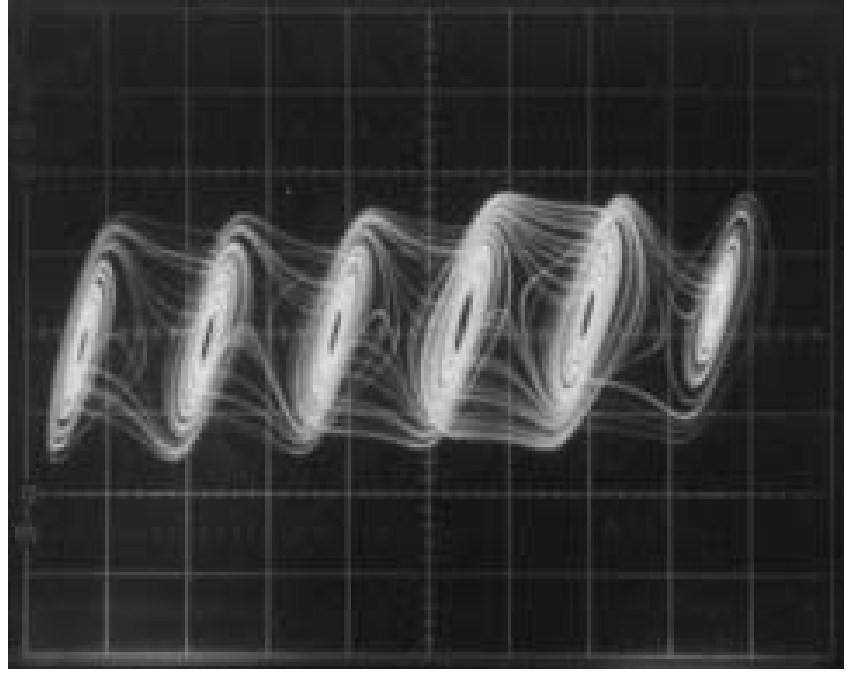

(a) 6-Scrolls

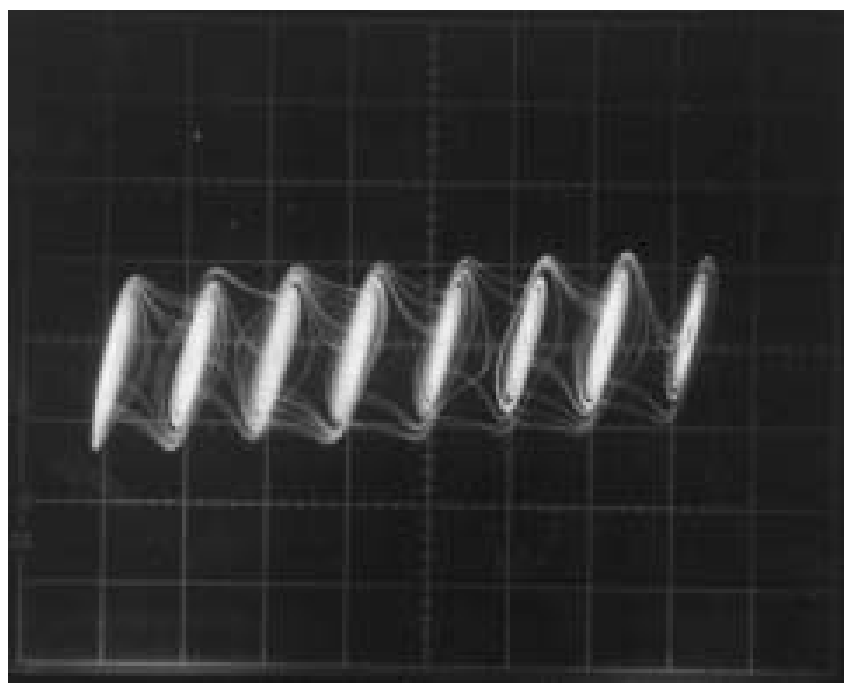

(c) 8-Scrolls

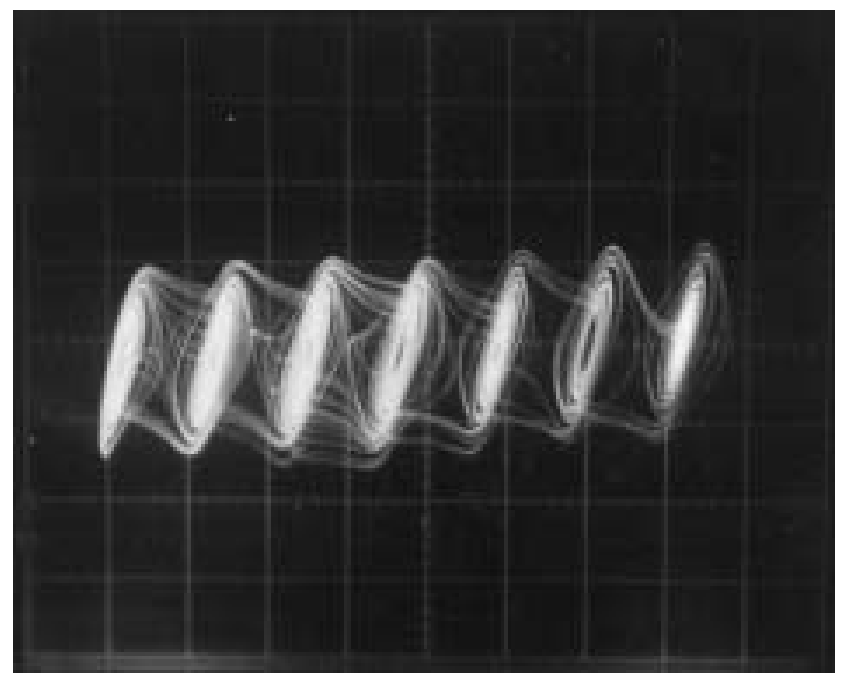

(b) 7-Scrolls

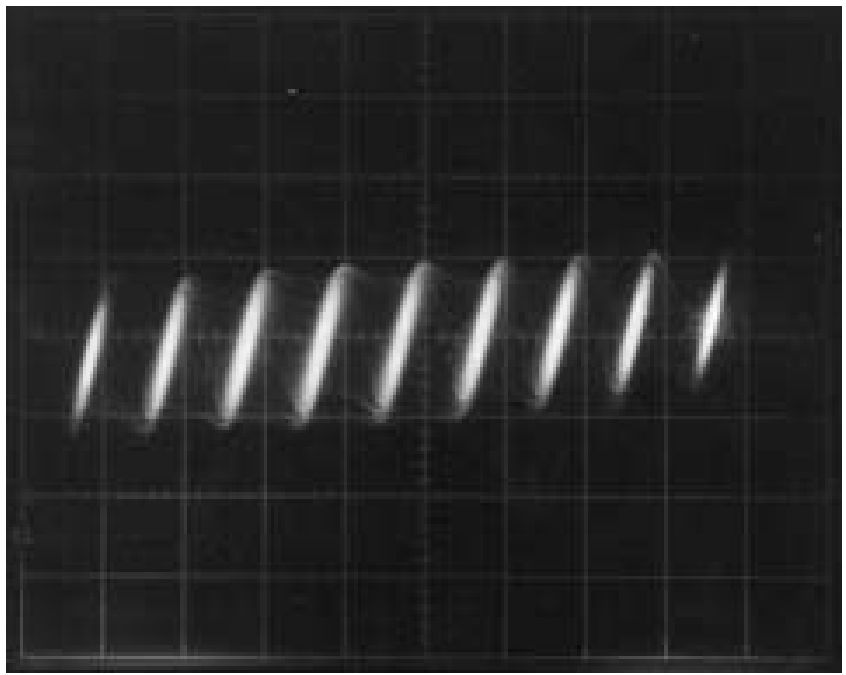

(d) 9-Scrolls

Fig. 26. Experimental observations of the sine function method.

circuit diagram of Fig. 28(b) is modified as shown in Fig. 29 [Salama et al., 2003]. Here, a high gain pushpull inverter composed of two MOS transistors is connected to create the sign function.

To generate $n \times m$-grid scroll chaotic attractors, Salama et al. [2003] further extended system (25), as follows:

$$
\left(\begin{array}{c}
\dot{x} \\
\dot{y} \\
\dot{z}
\end{array}\right)=\left(\begin{array}{ccc}
0 & 1 & 0 \\
0 & 0 & 1 \\
0 & -a & -a
\end{array}\right)\left(\begin{array}{l}
x \\
y \\
z
\end{array}\right)+\left(\begin{array}{c}
-a f(y) \\
0 \\
-a f(x)
\end{array}\right),
$$

where $f(x), f(y)$ are defined by (26) or (27). When $a=0.4$, system (28) with the sign nonlinearity (27) can generate a $2 \times 2$-grid scroll chaotic attractor, as shown in Fig. 30 [Salama et al., 2003].

In Fig. 28, the CFOA and the buffer are both realized by using the AD844 operational amplifiers. The NPN transistors are obtained from the LM3046 transistor array chips. The PNP transistors are all BC557. The circuit is supplied by $\pm 5 \mathrm{~V}$. All capacitors are taken as $3.3 \mathrm{nF}$ and $R_{2}=9 \mathrm{k} \Omega$, $R_{3}=2.2 \mathrm{k} \Omega$. The tail current $I_{S S}$ is set to $40 \mu \mathrm{A}$. The control voltages $\left(V_{1}, \ldots, V_{9}\right)$ are $(-500 \mathrm{mV}$, $-380 \mathrm{mV},-200 \mathrm{mV}, 0,200 \mathrm{mV}, 380 \mathrm{mV}, 500 \mathrm{mV}$, $640 \mathrm{mV}, 800 \mathrm{mV})$.

Figure 31(a) [Salama et al., 2003] displays the observed 5 -scroll chaotic attractor, generated by the 


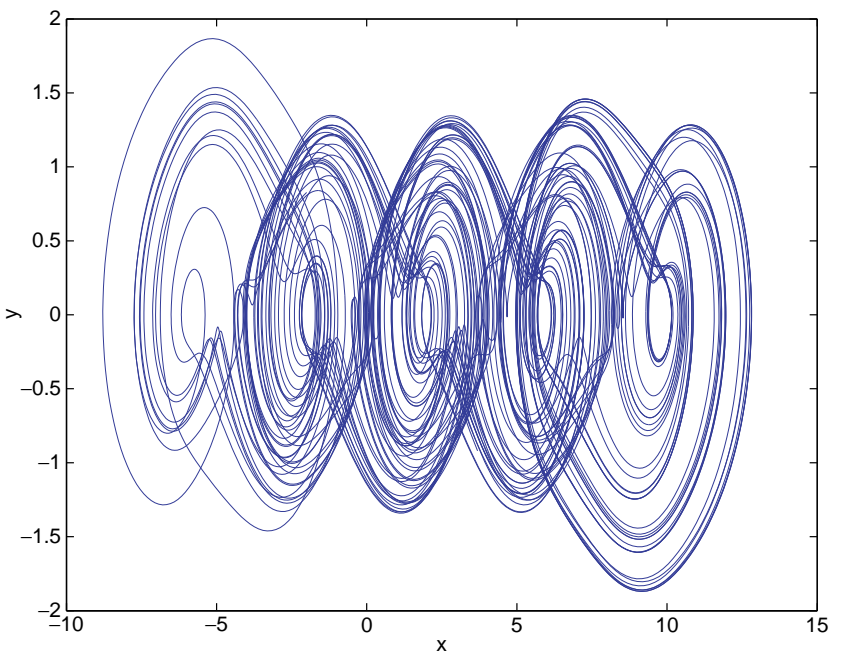

(a) 5-scroll attractor generated via the hyperbolic tangent function

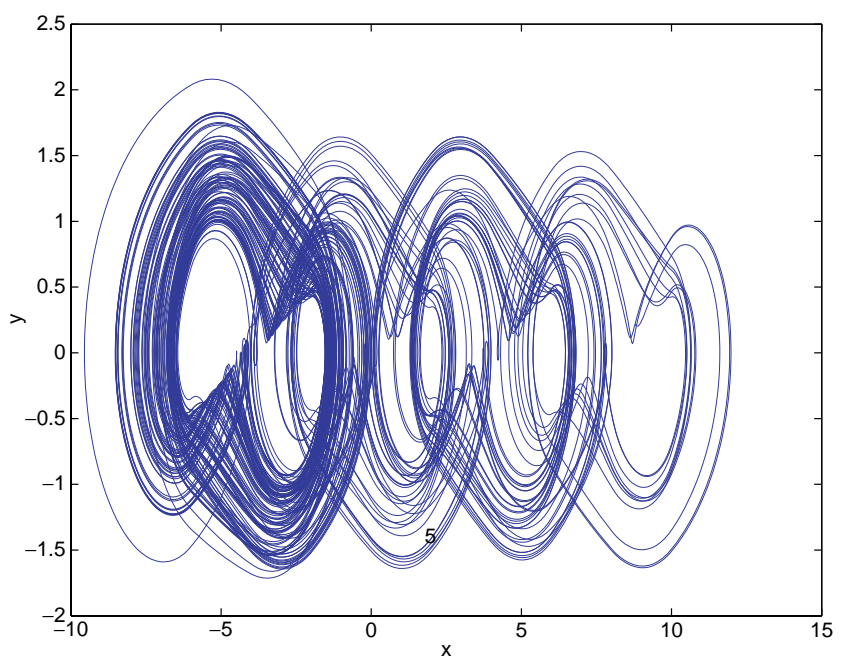

(b) 5-scroll attractor generated via the sign function

Fig. 27. 5-scroll chaotic attractors.

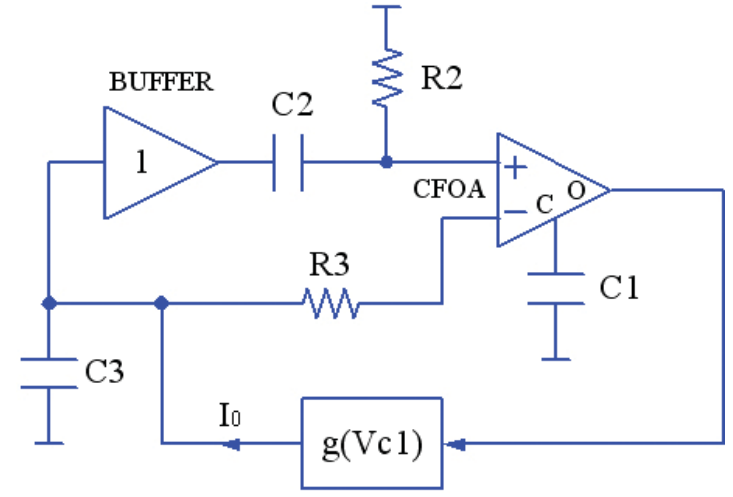

(a) Circuit diagram

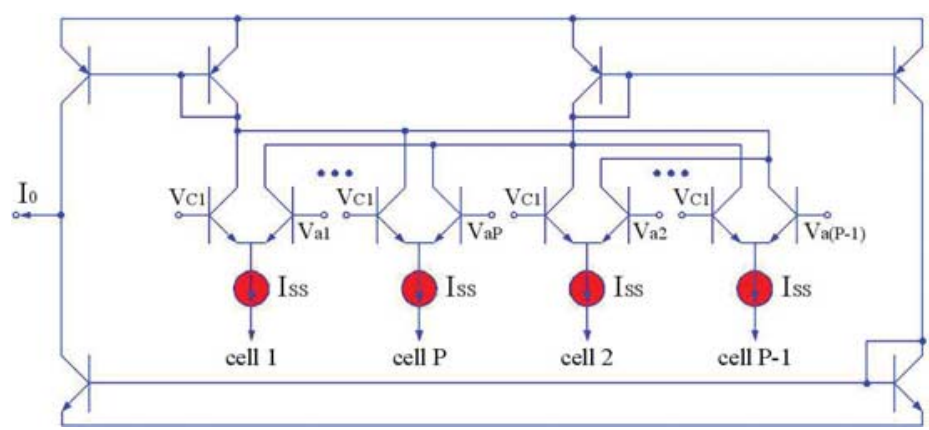

(b) Realization of $g\left(V_{C_{1}}\right)$

Fig. 28. Circuit diagram for generating $n$-scroll chaotic attractors [Özoğuz et al., 2002; Salama et al., 2003].

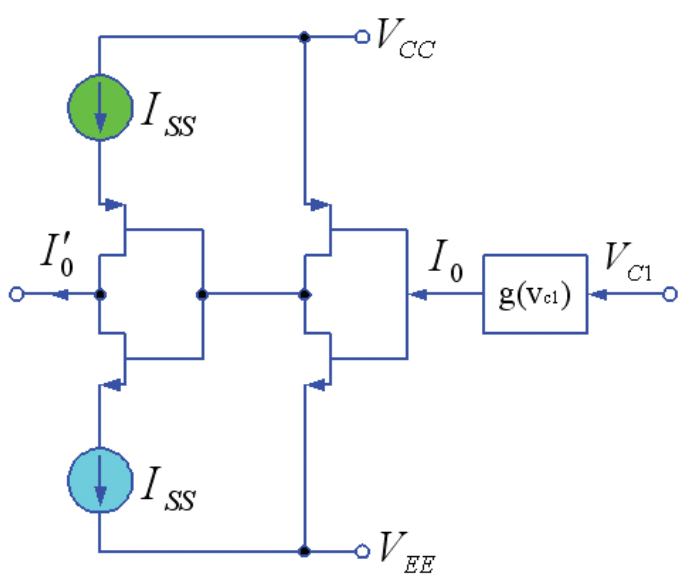

Fig. 29. Realization of the square-wave transconductor [Salama et al., 2003]. hyperbolic tangent nonlinear transconductor. Similarly, Fig. 31(b) [Salama et al., 2003] shows the observed 5-scroll chaotic attractor, generated by the sign nonlinear transconductor.

\subsection{General nonlinear modulating function approach}

This subsection presents a general nonlinear modulating function approach for generating $n$-scroll chaotic attractors from a general jerk circuit [Linz \& Sprott, 1999; Yu et al., 2005a, 2005d].

The general jerk circuit considered here is described by

$$
\dddot{x}+\beta \ddot{x}+\gamma \dot{x}=f(x),
$$




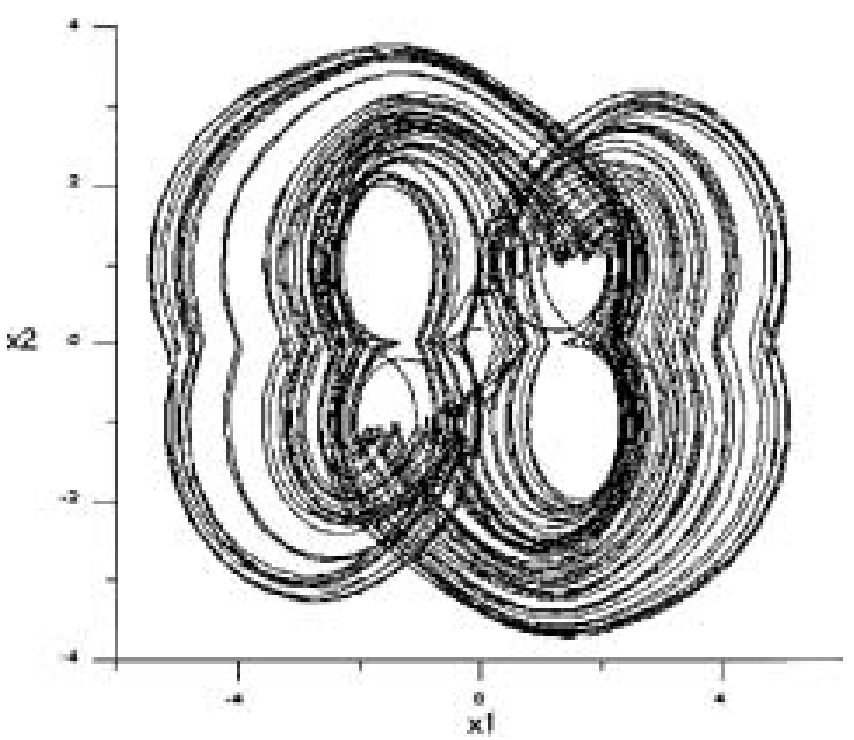

Fig. 30. $2 \times 2$-grid scroll chaotic attractor [Salama et al., 2003].

where $\beta, \gamma$ are real parameters, $f(x)$ is a nonlinear function, $\dot{x}=d x / d \tau$ is the velocity, $\ddot{x}=d^{2} x / d \tau^{2}$ is the acceleration, and $\dddot{x}=d^{3} x / d \tau^{3}$ is the jerk.

\subsubsection{Modulating function method}

To create $n$-scroll chaotic attractors from (29), Yu et al. [2005a, 2005d] constructed a swing modulating function of double sawtooth wave, which is described by

$$
f(x)=|F(x)| \operatorname{sgn}(x)-x,
$$

where $F(\cdot)$ may be an autonomous function or a nonautonomous function produced by outer signals. Of course, $F(\cdot)$ can be a constant in the simplest case. Here, assume that

$$
|F(\cdot)|=|A \sin (a x)|,
$$

where $A, a>0$ are parameters.

\subsubsection{Adjustable sawtooth wave function approach}

Yu et al. [2005a, 2005d] also applied the adjustable sawtooth wave to generate $n$-scroll chaotic attractors from (29). It can be classified into two cases with even and odd numbers of scrolls, respectively:

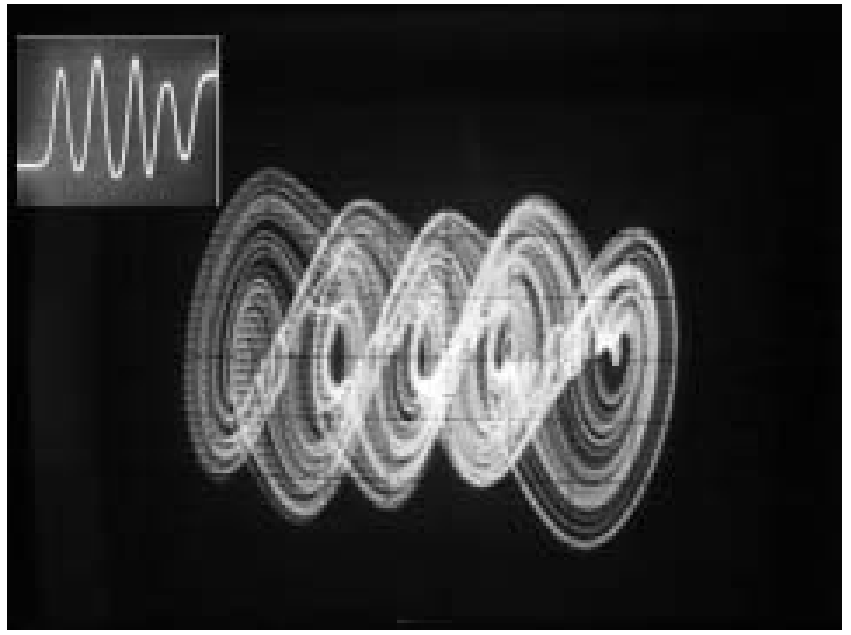

(a) $f(x)$ is given by $(26)$

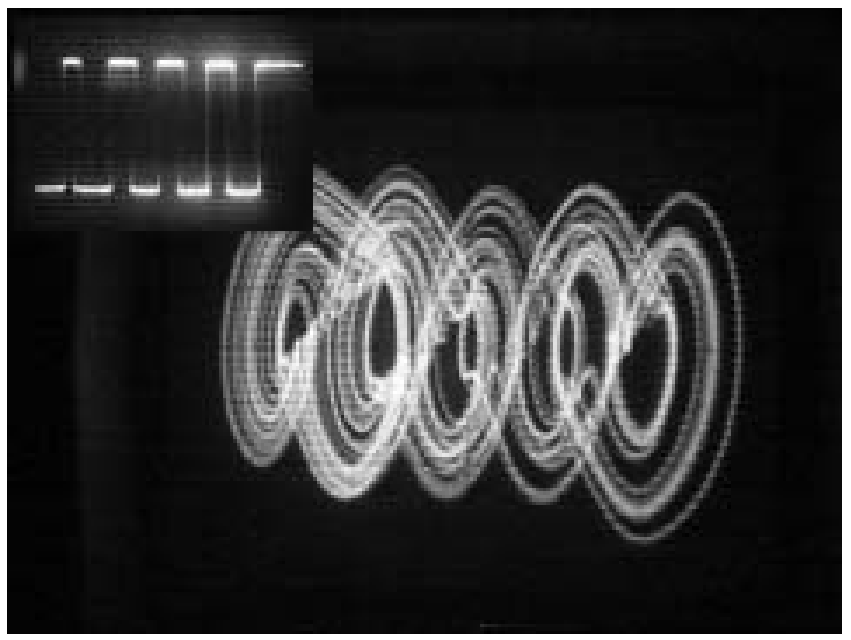

(b) $f(x)$ is given by $(27)$

Fig. 31. Experimental observations of $n$-scroll chaotic attractors ( $x$-axis: $200 \mathrm{mV} /$ div; $y$-axis: $50 \mathrm{mV} /$ div) [Salama et al., 2003].

(1) Even number of scrolls:

$$
\begin{aligned}
f_{1}(x)= & A_{0} \operatorname{sgn}(x) \\
& +\sum_{i=1}^{M}\left[\frac{A_{i-1}+A_{i}}{2} \operatorname{sgn}\left(x-\frac{2}{B} \sum_{j=0}^{i-1} A_{j}\right)\right] \\
& +\sum_{i=1}^{M}\left[\frac{A_{i-1}+A_{i}}{2} \operatorname{sgn}\left(x+\frac{2}{B} \sum_{j=0}^{i-1} A_{j}\right)\right] \\
& -B x,
\end{aligned}
$$

where all parameters $A_{i}>0(i=0,1,2, \ldots)$ and $B \in[0.7,1.2]$, which can generate $2 M+$ $2(M=1,2,3, \ldots)$ scrolls in the attractor. 
(2) Odd number of scrolls:

$$
\begin{aligned}
f_{2}(x)= & \sum_{i=1}^{M}\left\{\frac{A_{i-1}+A_{i}}{2}\right. \\
& \left.\times \operatorname{sgn}\left[x-\frac{1}{B}\left(2 \sum_{j=0}^{i-1} A_{j}-A_{0}\right)\right]\right\} \\
& +\sum_{i=1}^{M}\left\{\frac{A_{i-1}+A_{i}}{2}\right. \\
& \left.\times \operatorname{sgn}\left[x+\frac{1}{B}\left(2 \sum_{j=0}^{i-1} A_{j}-A_{0}\right)\right]\right\}-B x
\end{aligned}
$$

where all parameters $A_{i}>0(i=0,1,2, \ldots)$ and $B \in[0.7,1.2]$, which can create $2 M+$ $1(M=1,2,3, \ldots)$ scrolls in the attractor.

\subsubsection{Adjustable triangular wave function method}

Yu et al. [2005a, 2005d] furthermore introduced a PWL function with varying breakpoints and slopes to create $n$-scroll chaotic attractors from (29). It includes two cases with even and odd numbers of scrolls, respectively:

(1) Even number of scrolls:

$$
\begin{aligned}
f_{1}(x)= & \sum_{n=-M}^{M} \frac{A}{2 \alpha_{n}}\left[\left|\left(x-\frac{2 A n}{B}\right)+\alpha_{n}\right|\right. \\
& \left.-\left|\left(x-\frac{2 A n}{B}\right)-\alpha_{n}\right|\right]-B x
\end{aligned}
$$

where parameters $A>0,0.8 \leq B \leq 1.2, \alpha_{n} \in$ $(0,3 A / 10 B](n=0, \pm 1, \ldots, \pm M), M=1,2, \ldots$, which can create $2 M+2$ scrolls in the chaotic attractor.

(2) Odd number of scrolls:

$$
\begin{aligned}
f_{2}(x) & \sum_{\substack{n=-M \\
n \neq 0}}^{M} \frac{A}{2 \alpha_{n}}\left[\left|\left(x-\frac{A}{B}\left(2 n-\frac{|n|}{n}\right)\right)+\alpha_{n}\right|\right. \\
& \left.-\left|\left(x-\frac{A}{B}\left(2 n-\frac{|n|}{n}\right)\right)-\alpha_{n}\right|\right]-B x,
\end{aligned}
$$

where parameters $A>0,0.8 \leq B \leq 1.2, \alpha_{n} \in$ $(0,3 A / 10 B](n= \pm 1, \pm 2, \ldots, \pm M), M=1$, $2, \ldots$, which can create $2 M+1$ scrolls in the chaotic attractor.

\subsubsection{Adjustable transconductor wave function approach}

Yu et al. [2005a, 2005d] then proposed an adjustable transconductor wave method for generating $n$-scroll chaotic attractors from (29). It consists of two cases with even and odd numbers of scrolls, respectively:

(1) Even number of scrolls:

$$
f_{1}(x)=\sum_{n=-M}^{M} A \tanh \left[C_{n}\left(x-\frac{2 n A}{B}\right)\right]-B x,
$$

where $A, B, C_{n}$ are adjustable parameters and $M \in N$.

(2) Odd number of scrolls:

$$
\begin{aligned}
f_{2}(x)= & \sum_{\substack{n=-M \\
n \neq 0}}^{M} A \tanh \\
& \times\left[C_{n}\left(x-\left(2 n-\frac{|n|}{n}\right) \frac{A}{B}\right)\right]-B x,
\end{aligned}
$$

where $A, B, C_{n}$ are adjustable parameters and $M \in N$.

For simplicity, define the following notation: Type I: multiscroll attractors, with the sizes of the scrolls gradually increasing from the center to both sides; Type II: multiscroll attractors, with the sizes of the scrolls gradually decreasing from the center to both sides; Type III: multiscroll attractors, with the scrolls alternating between small and large scrolls; Type IV: multiscroll attractors, with all scrolls being same in size.

$\mathrm{Yu}$ et al. [2005a, 2005d] finally designed a circuit diagram to physically realize various multiscroll chaotic attractors. Figure 32 shows the circuitry. It includes five function parts; that is, Part I: integrator $N_{0}$; Part II: sawtooth wave and triangular wave generator $N_{1}$; Part III: buffer $N_{2}$; Part IV: switch linkages, including $K, K_{10}, K_{11}, K_{12}, K_{13}$, $K_{14}, K_{15}$; Part V: voltage-current conversion resistors $R 10 \sim R 15$.

Let $R_{3 j}=1 \mathrm{k} \Omega$ and $R_{2 j}=200 \mathrm{k} \Omega$ for $0 \leq j \leq 5$. When $K$ is switched on, $N_{1}$ generates a triangular wave; when $K$ is switched off, $N_{1}$ creates a sawtooth wave. All experimental parameters are given in [Yu et al., 2005a]. Figure 33 shows the experimental observations of 12 -scroll chaotic attractors with various sizes. 


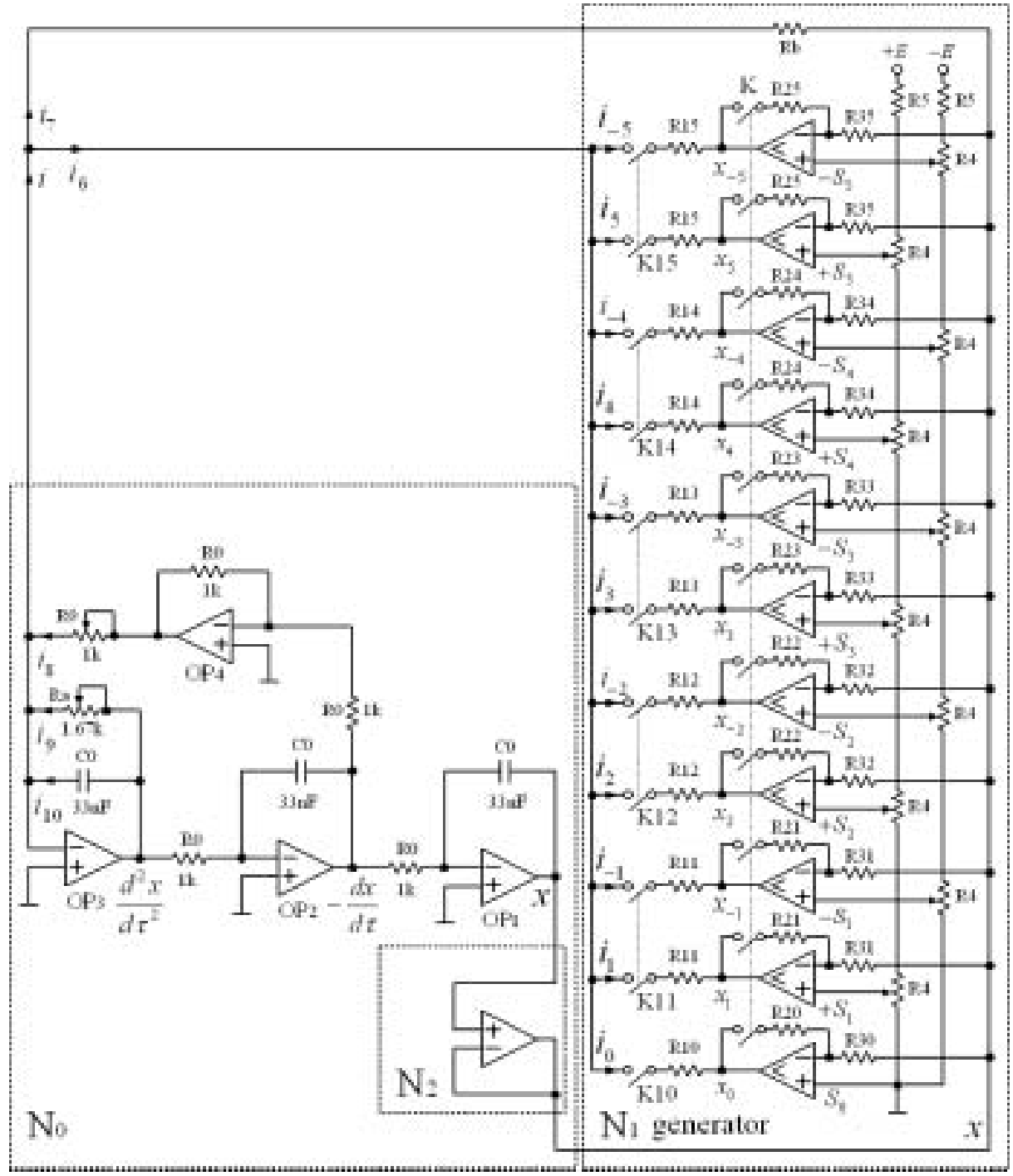

Fig. 32. Circuit diagram for generating $n$-scroll attractors.

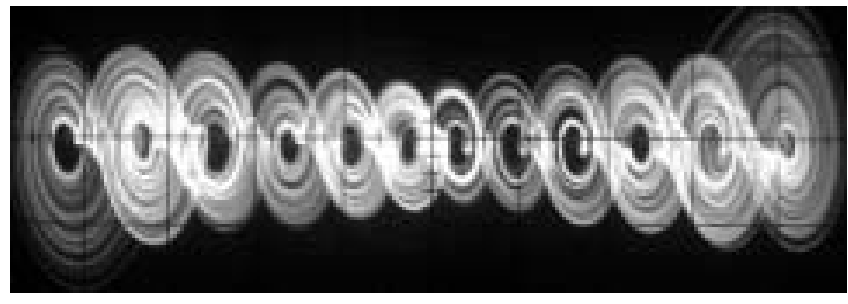

(a) Type I, $x=1.1 \mathrm{~V} / \operatorname{div}, y=0.4 \mathrm{~V} / \operatorname{div}$

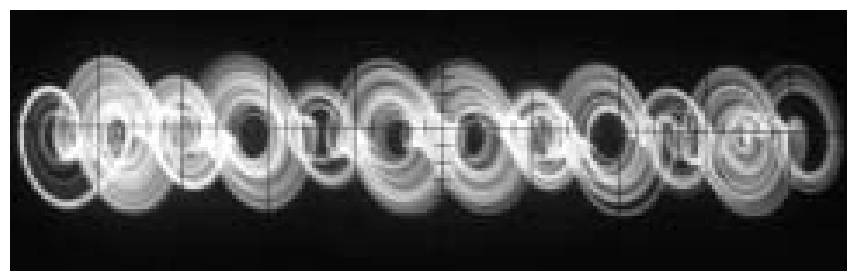

(c) Type III, $x=0.8 \mathrm{~V} / \operatorname{div}, y=0.4 \mathrm{~V} / \operatorname{div}$

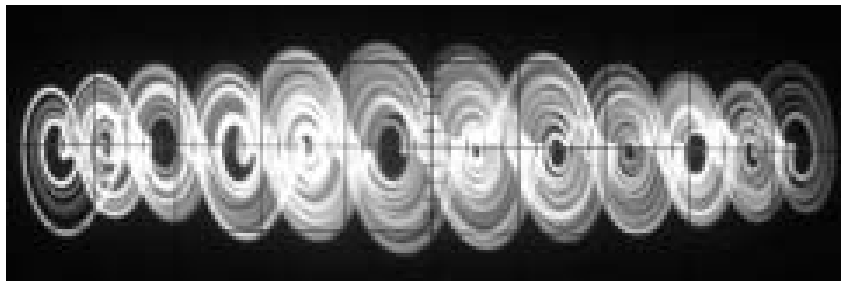

(b) Type II, $x=1.05 \mathrm{~V} / \operatorname{div}, y=0.5 \mathrm{~V} / \operatorname{div}$

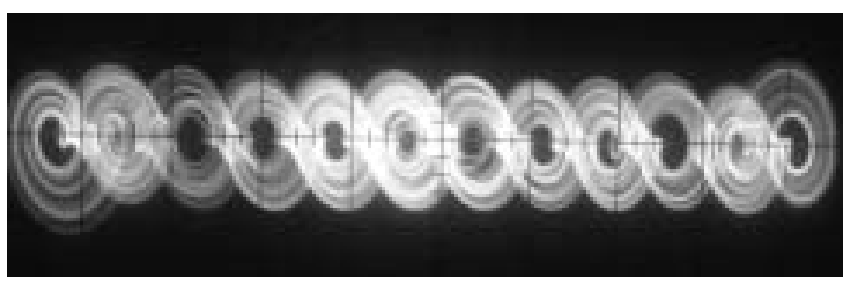

(d) Type IV $, x=0.66 \mathrm{~V} / \operatorname{div}, y=0.33 \mathrm{~V} / \operatorname{div}$

Fig. 33. Experimental observations of 12-scroll chaotic attractors. 
Moreover, the systematic nonlinear modulating function methodology reviewed here can be used to design the swings, widths, slopes, breakpoints, equilibriums and shapes of the $n$-scroll chaotic attractors, as preferred, via the adjustable sawtooth wave, triangular wave and transconductor wave functions.

\section{Design of Multidirectional Multiscroll Chaotic Attractors via Basic Circuits}

It is well known that step circuit, hysteresis circuit and saturated circuit are the three types of basic circuits. This section introduces several approaches for generating multidirectional multiscroll chaotic attractors by using these basic circuits.

\subsection{Step function approach}

Yalcin et al. [2002b] constructed a new family of scroll and grid-scroll attractors by using the step circuit, including 1-D $n$-scroll, 2 -D $n \times m$-grid scroll, and 3 -D $n \times m \times l$-grid scroll chaotic attractors. The state equation of this family of systems is given by

$$
\dot{\mathbf{X}}=\mathbf{A X}+\mathbf{B} \sigma(\mathbf{C X}),
$$

where $\mathbf{X}=(x, y, z)^{T}$ and

$$
\begin{gathered}
\mathbf{A}=\left(\begin{array}{ccc}
0 & 1 & 0 \\
0 & 0 & 1 \\
-a & -a & -a
\end{array}\right), \quad \mathbf{B}=\left(\begin{array}{ccc}
b_{y} & 0 & 0 \\
0 & b_{z} & 0 \\
0 & 0 & a
\end{array}\right), \\
\mathbf{C}=\left(\begin{array}{lll}
0 & 1 & 0 \\
0 & 0 & 1 \\
1 & 0 & 0
\end{array}\right) .
\end{gathered}
$$

There are three different cases:

(i) 1-D $n$-scroll chaotic attractors:

$$
\left\{\begin{array}{l}
b_{y}=b_{z}=0 \\
\sigma(\cdot)=\left(\begin{array}{c}
0 \\
0 \\
f_{1}(\cdot)
\end{array}\right)
\end{array}\right.
$$

where

$$
f_{1}(x)=\sum_{i=1}^{M_{x}} g_{\frac{-2 i+1}{2}}(x)+\sum_{i=1}^{N_{x}} g_{\frac{2 i-1}{2}}(x),
$$

and

$$
g_{\theta}(\zeta)=\left\{\begin{array}{lll}
1, & \zeta \geq \theta & \theta>0 \\
0, & \zeta<\theta & \theta>0 \\
0, & \zeta \geq \theta & \theta<0 \\
-1, & \zeta<\theta & \theta<0
\end{array}\right.
$$

which belongs to the sector $[0,2]$.

(ii) 2-D $n \times m$-grid scroll chaotic attractors:

$$
\left\{\begin{array}{l}
b_{y}=-1, \quad b_{z}=0, \\
\sigma(\cdot)=\left(\begin{array}{c}
f_{1}(\cdot) \\
0 \\
f_{2}(\cdot)
\end{array}\right)
\end{array}\right.
$$

where $f_{1}(\cdot)$ is defined by $(32)$,

$$
f_{2}(x)=\sum_{i=1}^{m-1} \beta g_{p_{i}}(x),
$$

and

$$
\begin{aligned}
p_{i} & =M_{y}+0.5+(i-1)\left(M_{y}+N_{y}+1\right), \\
\beta & =M_{y}+N_{y}+1,
\end{aligned}
$$

which belongs to the sector $\left[0,\left(\left(M_{y}+N_{y}+1\right) /\right.\right.$ $\left.\left.\left(M_{y}+0.5\right)\right)\right]$.

(iii) 3-D $n \times m \times l$-grid scroll chaotic attractors:

$$
\left\{\begin{array}{l}
b_{y}=-1, \quad b_{z}=-1, \\
\sigma(\cdot)=\left(\begin{array}{l}
f_{1}(\cdot) \\
f_{1}(\cdot) \\
f_{3}(\cdot)
\end{array}\right)
\end{array}\right.
$$

where $f_{1}(\cdot)$ is defined by $(32)$,

$$
f_{3}(x)=\sum_{i=1}^{k-1} \gamma g_{n_{l}}(x),
$$

and

$n_{l}=\rho+0.5+(l-1)(\rho+\zeta+1), \quad \gamma=\rho+\zeta+1$,

with

$$
\begin{aligned}
& \rho=\left|\min _{i, j}\left\{u_{i}^{e q, y}+u_{j}^{e q, z}\right\}\right|, \\
& \zeta=\left|\max _{i, j}\left\{u_{i}^{e q, y}+u_{j}^{e q, z}\right\}\right|,
\end{aligned}
$$

and $u_{i}^{e q, y}, u_{j}^{e q, z}$ are the vectors for the $y$ and $z$ variables related to the equilibrium points, respectively, which belong to the sector $[0,((\zeta+\rho+1) /(\rho+0.5))]$.

Figure 34(a) [Yalcin et al., 2002b] shows a 10-scroll chaotic attractor, where $M_{x}=0, N_{x}=1$, 


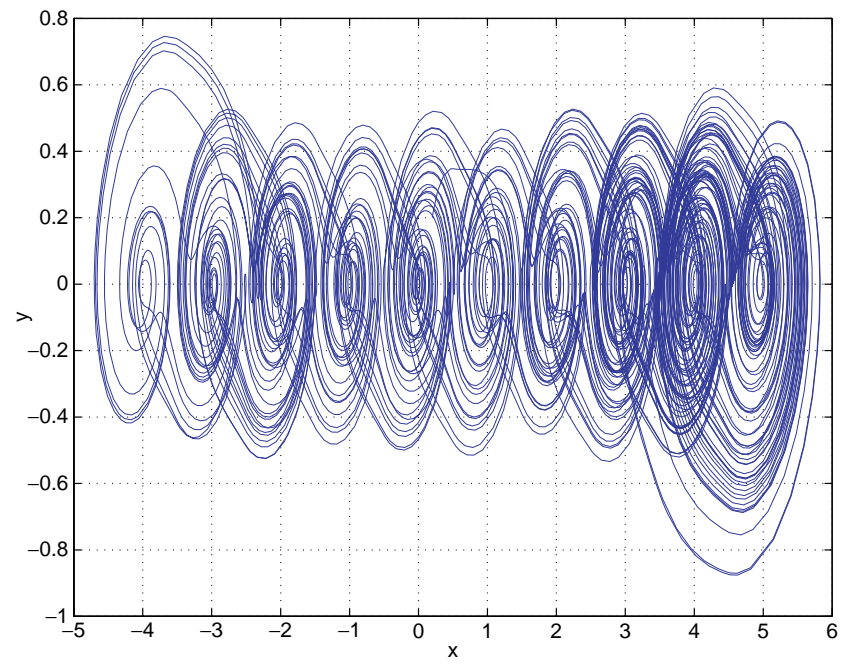

(a) 1-D 10-scroll attractor

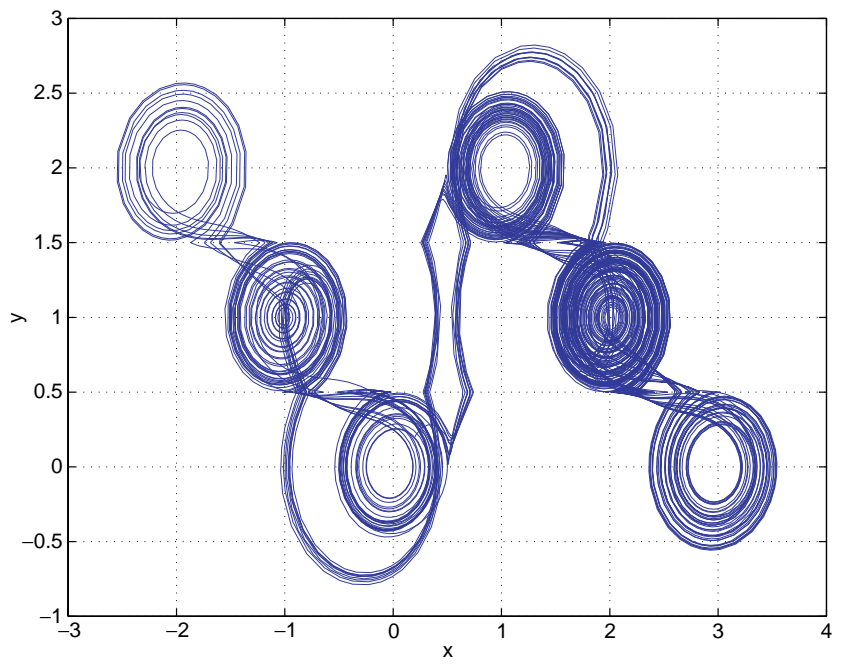

(b) 2 -D $2 \times 3$-grid scroll attractor

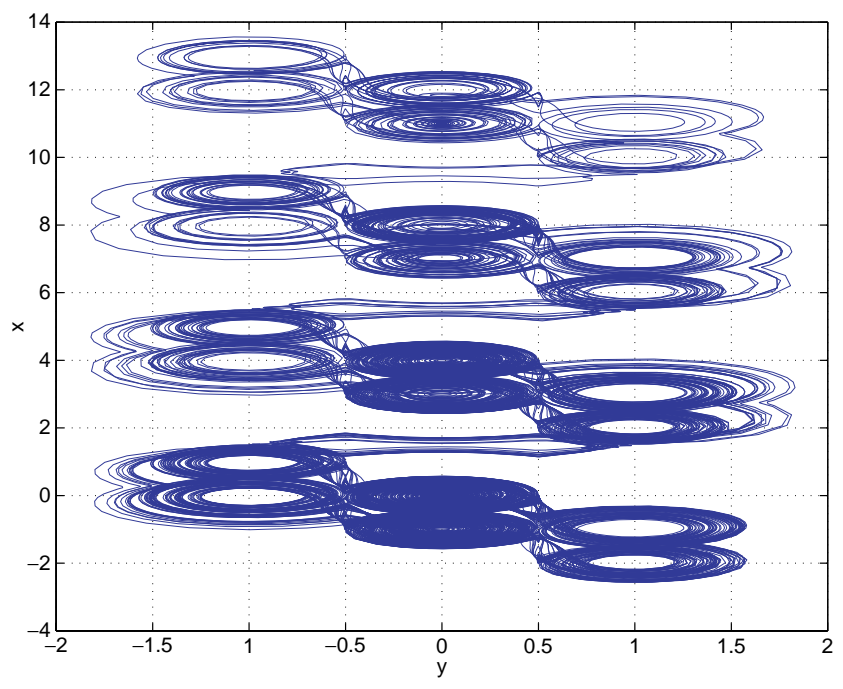

(c) 3 -D $4 \times 3 \times 2$-grid scroll attractor

Fig. 34. Numerical simulations of generating multiscroll chaotic attractors via step circuit [Yalcin et al., 2002b].

$a=0.8, M=4, N=5, X_{0}=[0.6721$ 0.8381 0.0196]. Figure 34(b) [Yalcin et al., 2002b] displays a $2 \times 3$-grid scroll chaotic attractor, where $M_{y}=0, N_{y}=2, m=2$. Figure 34(c) [Yalcin et al., 2002 b] shows a $4 \times 3 \times 2$-grid scroll chaotic attractor, where $M_{y}=1, N_{y}=1, M_{x}=0, N_{x}=1, k=4$.

Yalcin et al. [2002b] also designed a circuit diagram for experimentally verifying the multiscroll chaotic attractors. Figure 35 shows the circuit diagram. The subcircuits within the dashed lines located at the upper-left, upper-right, and lower positions can generate multiscroll attractors in $x, y, z$-directions, respectively. CFOAs are implemented using AD844 from analog circuits where the types of comparators are LM311. The voltages of electronic sources are $\pm 15 \mathrm{~V}$ and $C_{1}=$ $C_{2}=C_{3}=1 \mathrm{nF}, R_{1}=5.1 \mathrm{k} \Omega$. When $R_{2}=$ $R_{4}=8 \mathrm{k} \Omega, R_{x 1}=R_{x 2}=R_{x 3}=R_{x 4}=70 \mathrm{k} \Omega$, the circuit diagram, modified by removing the subcircuits within the dashed lines located at upperright and lower positions, and adding two more comparators in the subcircuit within the dashed lines located at the upper-left position, can create a 5-scroll chaotic attractor, as shown in Fig. 36(a) [Yalcin et al., 2002b]. When $R_{2}=R_{4}=12 \mathrm{k} \Omega$, $R_{x 1}=28 \mathrm{k} \Omega, R_{x 2}=30 \mathrm{k} \Omega, R_{y 1}=90 \mathrm{k} \Omega, R_{y 2}=$ $80 \mathrm{k} \Omega$, the circuit diagram, modified by removing the subcircuit within the dashed lines located at 


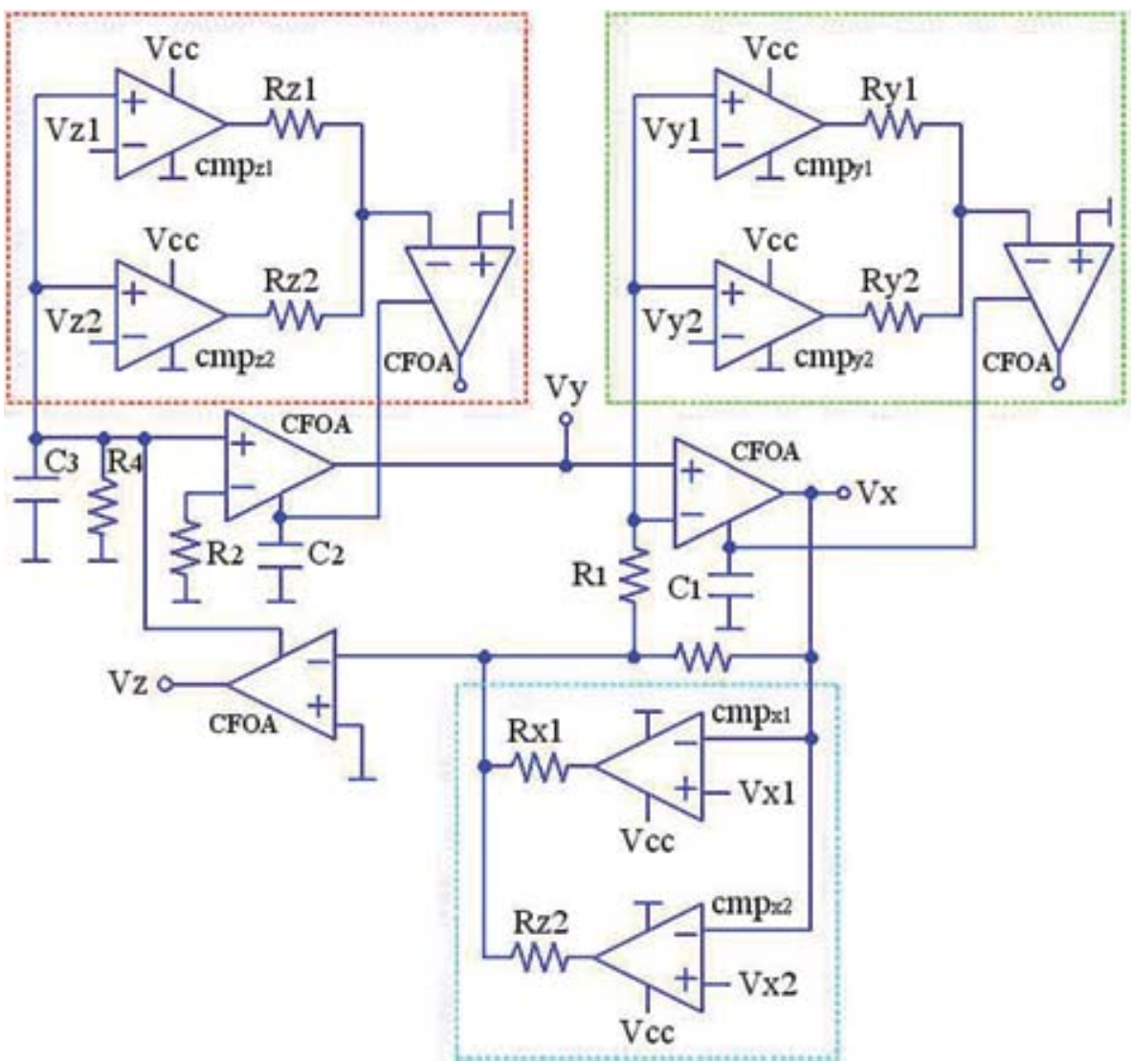

Fig. 35. Circuit diagram for realizing multiscroll attractors.

the lower position, and adding one comparator in the subcircuit within the dashed lines located at the upper-right position, can generate a $3 \times 3$ scroll chaotic attractor, as shown in Fig. 36(b) [Yalcin et al., 2002b]. When $R_{2}=R_{4}=8.3 \mathrm{k} \Omega$, $R_{x 1}=19 \mathrm{k} \Omega, R_{y 1}=47 \mathrm{k} \Omega, R_{z 1}=50 \mathrm{k} \Omega$, the circuit diagram, modified by removing the comparators comp $x_{x 2}, \operatorname{comp}_{y 2}$ and $\operatorname{comp}_{z 2}$ in the subcircuits, can generate a $2 \times 2 \times 2$-scroll chaotic attractor, as shown in Fig. 36(c) [Yalcin et al., 2002b].

\subsection{Hysteresis series method}

In this subsection, a systematic approach is introduced for generating multidirectional multiscroll chaotic attractors by using hysteresis series. It includes two cases: the system to be controlled is a 2D linear autonomous system, and is a three-dimensional (3D) linear autonomous system, respectively.

\subsubsection{Two-dimensional hysteresis system}

In the following, a design method is described for creating multiscroll chaotic attractors from a $2 \mathrm{D}$ linear autonomous system via hysteresis series. It can generate 1-D $n$-scroll and 2-D $n \times m$-grid scroll chaotic attractors.

Han et al. [2005] proposed a 2D hysteresis multiscroll chaotic system, described by

$$
\dot{\mathbf{X}}=\mathbf{A X}+\mathbf{B} \theta(\mathbf{C X})
$$

where $\mathbf{X}=(x, y)^{T}$ and

$$
\begin{aligned}
& \mathbf{A}=\left(\begin{array}{cc}
0 & 1 \\
-a & b
\end{array}\right), \quad \mathbf{B}=\left(\begin{array}{cc}
-1 & 0 \\
-b & a
\end{array}\right), \\
& \mathbf{C}=\left(\begin{array}{ll}
0 & 1 \\
1 & 0
\end{array}\right) .
\end{aligned}
$$

There are three different cases outlined in the following:

(i) 1-D horizontal $n$-scroll chaotic attractors:

$$
\theta(\cdot)=\left(\begin{array}{c}
0 \\
h\left(x, p_{1}, q_{1}\right)
\end{array}\right)
$$




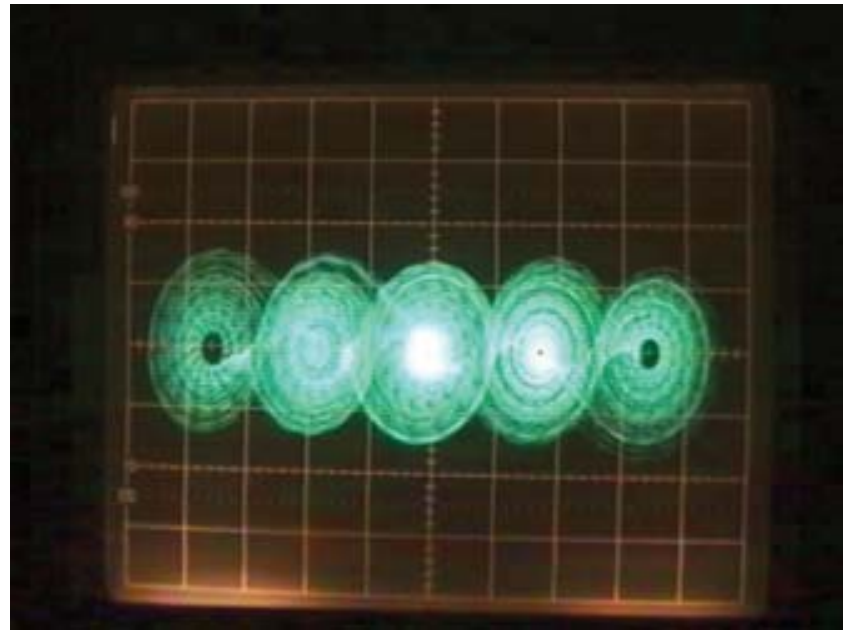

(a) 1-D 5-scroll $(a=0.64, x=0.5 \mathrm{~V} / \mathrm{div}, y=0.5 \mathrm{~V} / \mathrm{div})$

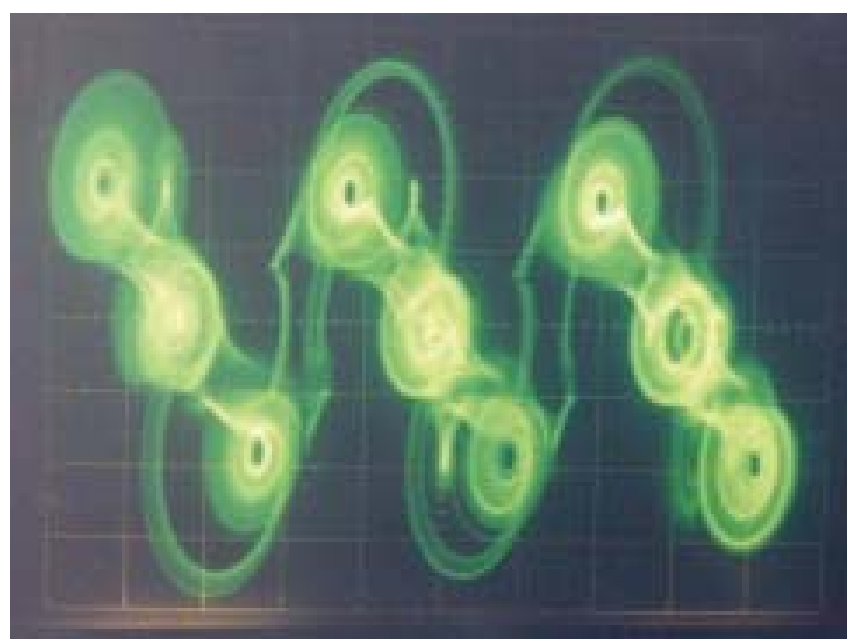

(b) 2 -D $3 \times 3$-grid scroll $(x=1 \mathrm{~V} /$ div, $y=0.5 \mathrm{~V} /$ div $)$

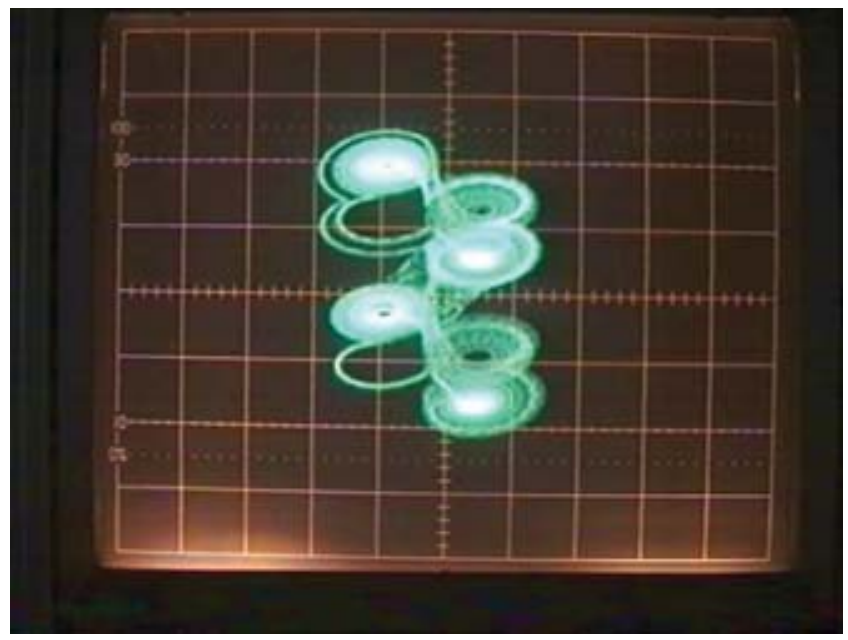

(c) 3 -D $2 \times 2 \times 2$-grid scroll $(x=1 \mathrm{~V} /$ div, $y=2 \mathrm{~V} /$ div $)$

Fig. 36. Experimental observations of multiscroll chaotic attractors generated via step circuit [Yalcin et al., 2002b]. where the hysteresis series function $h\left(x, p_{1}, q_{1}\right)$ is defined by

$$
\begin{aligned}
& h\left(x, p_{1}, q_{1}\right) \\
& \quad= \begin{cases}-p_{1} & \text { if } x<-p_{1}+1 \\
i & \text { if } \begin{array}{l}
i-1<x<i+1 \\
i=-p_{1}+1, \ldots, q_{1}-1
\end{array} \\
q_{1} & \text { if } x>q_{1}-1 .\end{cases}
\end{aligned}
$$

Figure 37 shows the phase portrait of the hysteresis series. Here, all equilibria are located in the $x$-axis.

(ii) 1 -D vertical $n$-scroll chaotic attractors:

$$
\theta(\cdot)=\left(\begin{array}{c}
h\left(y, p_{2}, q_{2}\right) \\
0
\end{array}\right)
$$

where $h\left(y, p_{2}, q_{2}\right)$ is similarly defined by (34), whose corresponding equilibria are located in the $y$-axis.

(iii) 2-D $n \times m$-grid scroll chaotic attractors:

$$
\theta(\cdot)=\left(\begin{array}{c}
h\left(y, p_{2}, q_{2}\right) \\
h\left(x, p_{1}, q_{1}\right)
\end{array}\right)
$$

where $h\left(x, p_{1}, q_{1}\right), \quad h\left(y, p_{2}, q_{2}\right)$ are similarly defined by (34), whose corresponding equilibria are integer points in the $x-y$ plane.

When $a=1, b=0.125, p=q=3$, system (33) has a 1-D horizontal 7-scroll chaotic attractor, as shown in Fig. 38(a); when $a=1, b=0.125$, $p=q=3$, system (33) has a 1 -D vertical 7 -scroll chaotic attractor, as shown in Fig. 38(b); when $a=1, b=0.125, p_{1}=q_{1}=3, p_{2}=q_{2}=1$, system (33) has a 2-D $7 \times 3$-grid scroll chaotic attractor, as shown in Fig. 38(c). Moreover, one can arbitrarily

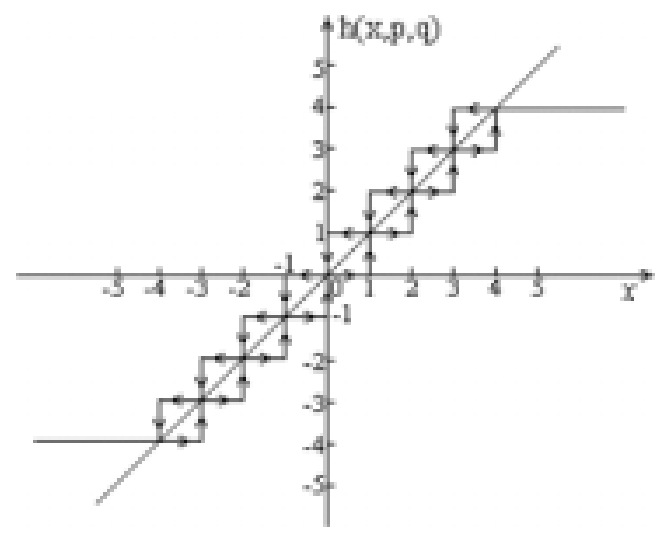

Fig. 37. Hysteresis series. 


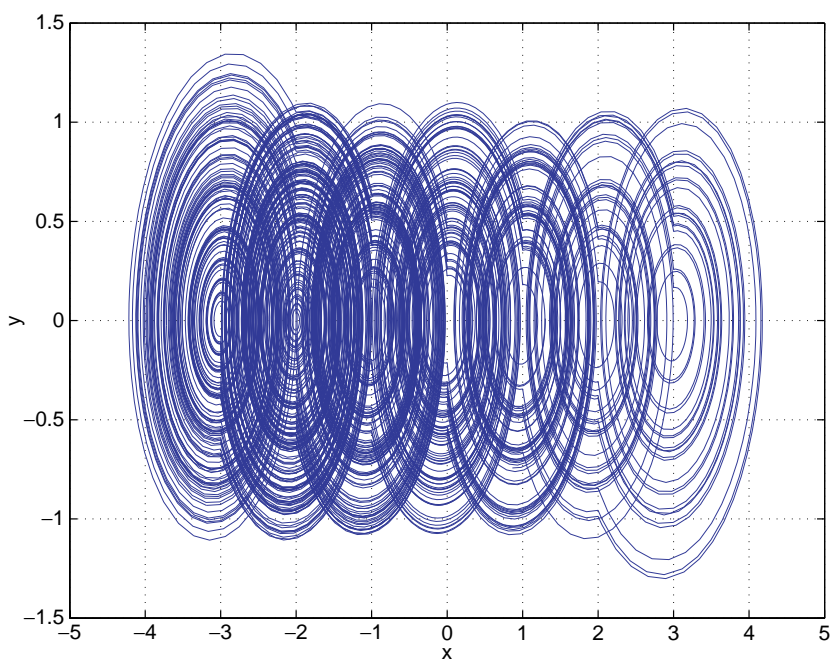

(a) 1-D horizontal 7-scroll attractor

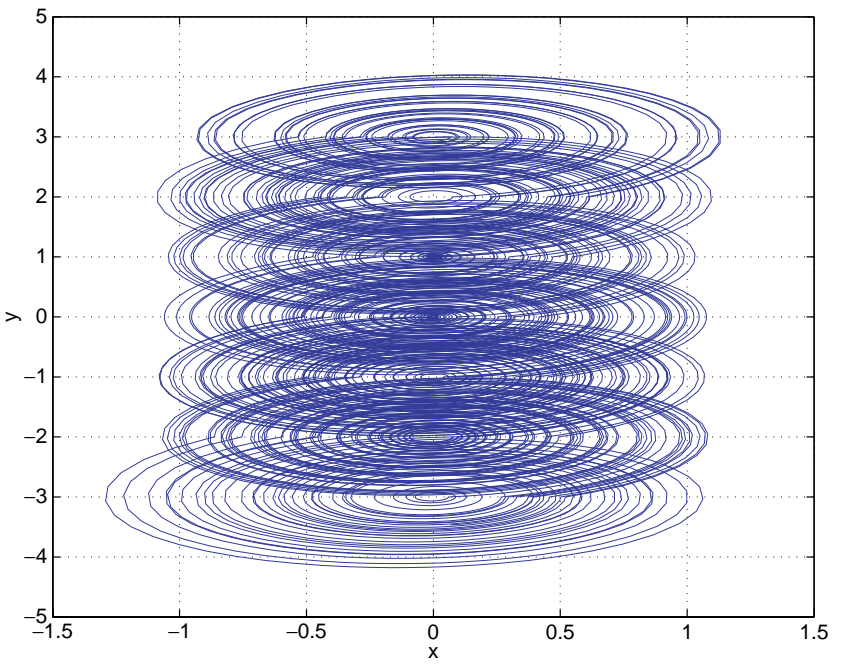

(b) 1-D vertical 7-scroll attractor

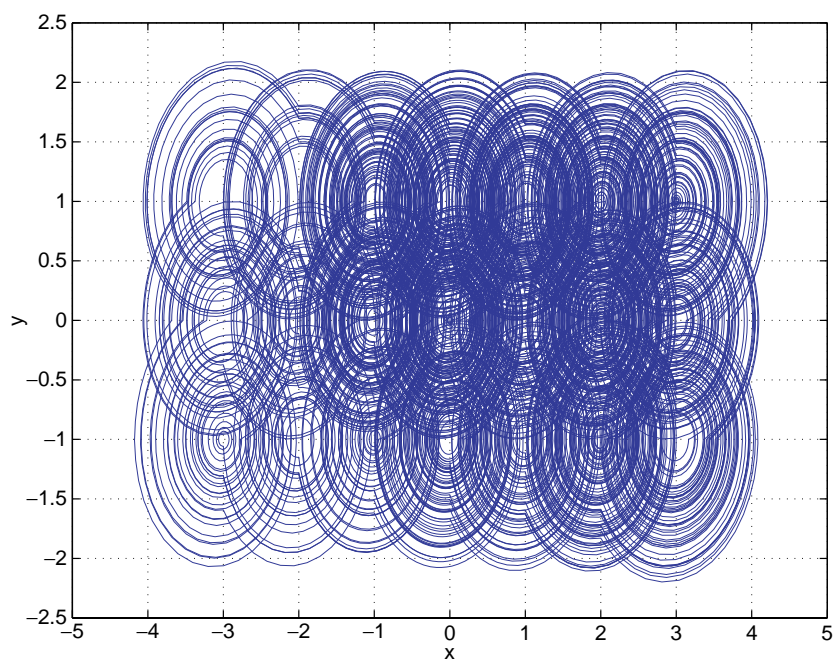

(c) 2 -D $7 \times 3$-grid scroll attractor

Fig. 38. Hysteresis chaotic attractors generated from a 2D linear autonomous system.

design the numbers and also the positions as well as the orientations of the scrolls of the hysteresis chaotic system (33).

Figure 39 displays the circuitry for physically realizing $n \times m$-grid scroll chaotic attractors. It consists of two function parts: (a) hysteresis series building block; (b) the second-order system [Han et al., 2005]. The circuit parameters are: $R 1=R 2=$ $R 4=R 7=R 9=R 12=10 \mathrm{k} \Omega, R 5=R 10=$ $R 25=R 26=100 \mathrm{k} \Omega, R 22=R 23=R 28=220 \mathrm{k} \Omega$, $R 21=R 24=3.3 \mathrm{M} \Omega, R 3, R 8$ and $R 11$ are potentiometers of $50 \mathrm{k} \Omega, 50 \mathrm{k} \Omega$ and $20 \mathrm{k} \Omega$, respectively,
$V R 1, V R 2$ and $V R 3$ are potentiometers of $2 \mathrm{M} \Omega$, $300 \mathrm{k} \Omega$ and $300 \mathrm{k} \Omega$, respectively, $C 1=0.01 \mu F$, and $C 2=0.001 \mu F$. D1 and D2 are diodes. All the operational amplifiers are LM324. Figure 40 shows a 2 -D $5 \times 3$-grid scroll chaotic attractor generated by this circuit.

\subsubsection{Three-dimensional hysteresis system}

Here, a new systematic approach is introduced for generating multidirectional multiscroll chaotic attractors from a 3D linear autonomous system 


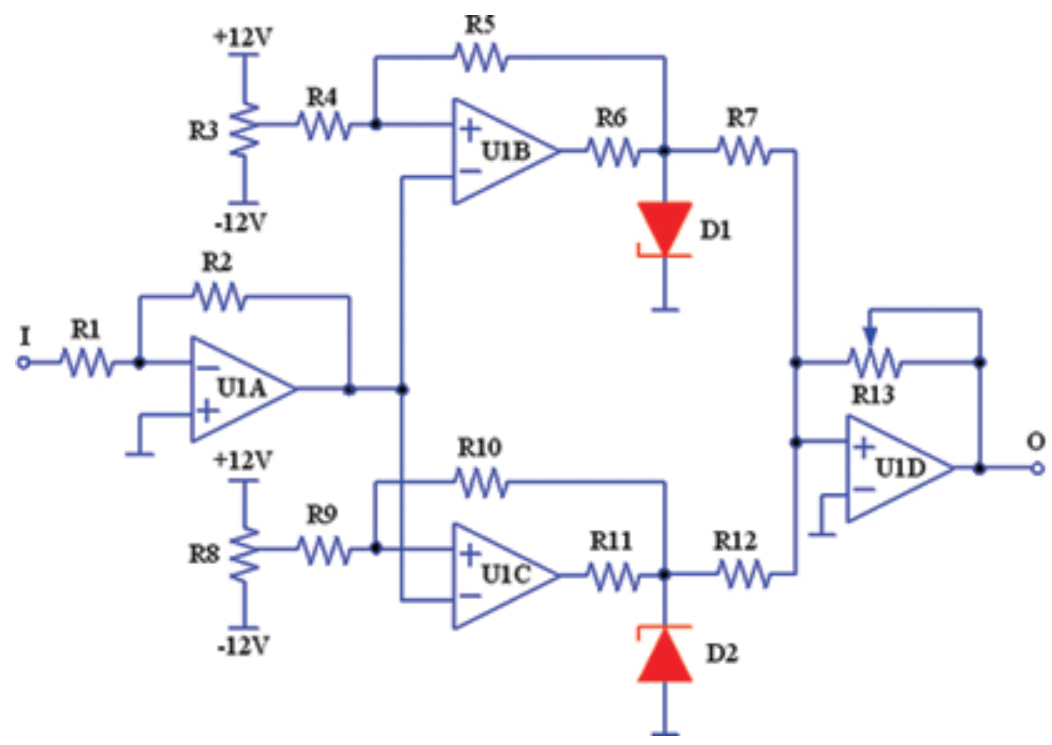

(a) Hysteresis series building block

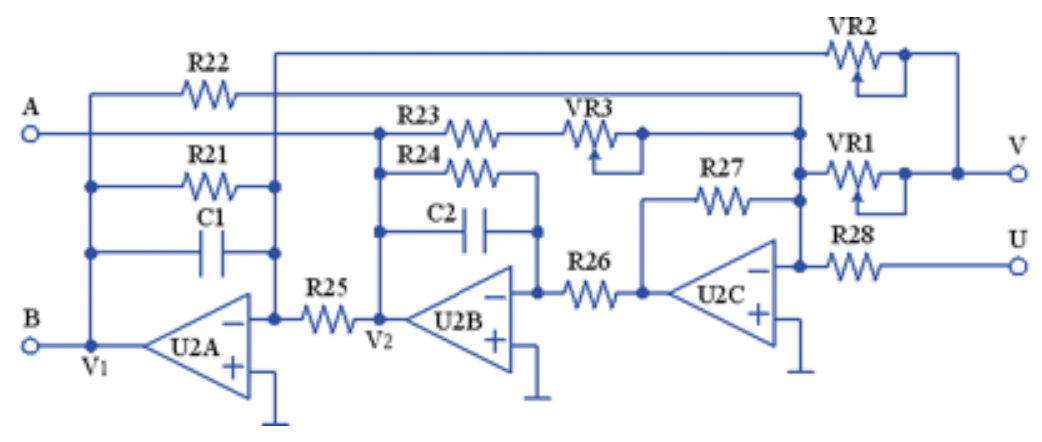

(b) Second-order system

Fig. 39. Circuitry for realizing $n \times m$-grid scroll chaotic attractors.

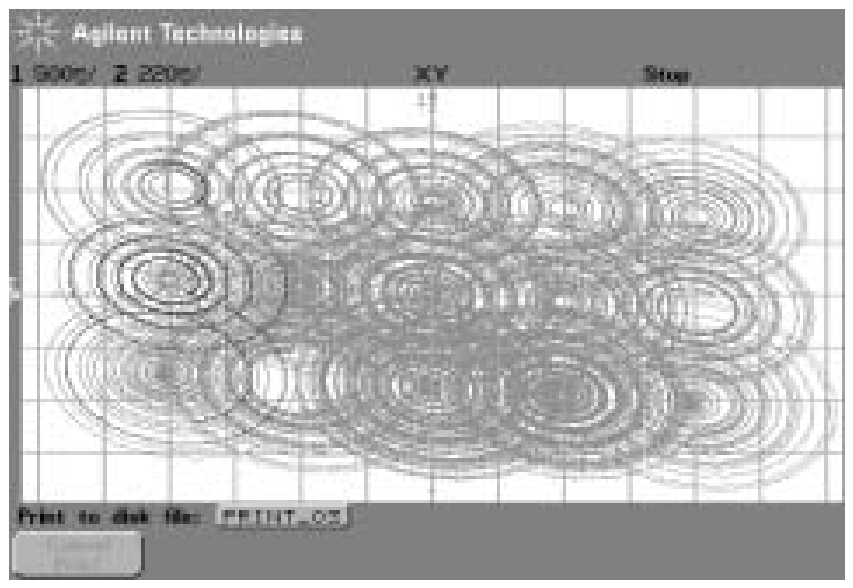

Fig. 40. Observed $5 \times 3$-grid scroll chaotic attractor.

using hysteresis series [Lü et al., 2003b, 2004d, 2005a, 2005b, 2005c]. This includes 1-D n-scroll,
2-D $n \times m$-grid scroll, and 3-D $n \times m \times l$-grid scroll chaotic attractors. The system is described by [Lü et al., 2004d]

$$
\dot{\mathbf{X}}=\mathbf{A X}+\mathbf{B} \theta(\mathbf{X}),
$$

where $\mathbf{X}=(x, y, z)^{T}$ is the state vector, $\mathbf{B}=-\mathbf{A}$, and

$$
\mathbf{A}=\left(\begin{array}{ccc}
0 & 1 & 0 \\
0 & 0 & 1 \\
-a & -b & -c
\end{array}\right)
$$

There are three different cases to consider:

(i) 1-D hysteresis $n$-scroll chaotic attractors:

$$
\theta(\mathbf{X})=\left(\begin{array}{c}
h\left(x, p_{1}, q_{1}\right) \\
0 \\
0
\end{array}\right),
$$


where the hysteresis series function $h\left(x, p_{1}, q_{1}\right)$ is given by (34).

(ii) 2 -D hysteresis $n \times m$-grid scroll chaotic attractors:

$$
\theta(\mathbf{X})=\left(\begin{array}{c}
h\left(x, p_{1}, q_{1}\right) \\
h\left(y, p_{2}, q_{2}\right) \\
0
\end{array}\right),
$$

where the hysteresis series functions $h\left(x, p_{1}\right.$, $\left.q_{1}\right)$ and $h\left(y, p_{2}, q_{2}\right)$ are similarly defined by (34).

(iii) 3 -D hysteresis $n \times m \times l$-grid scroll chaotic attractors:

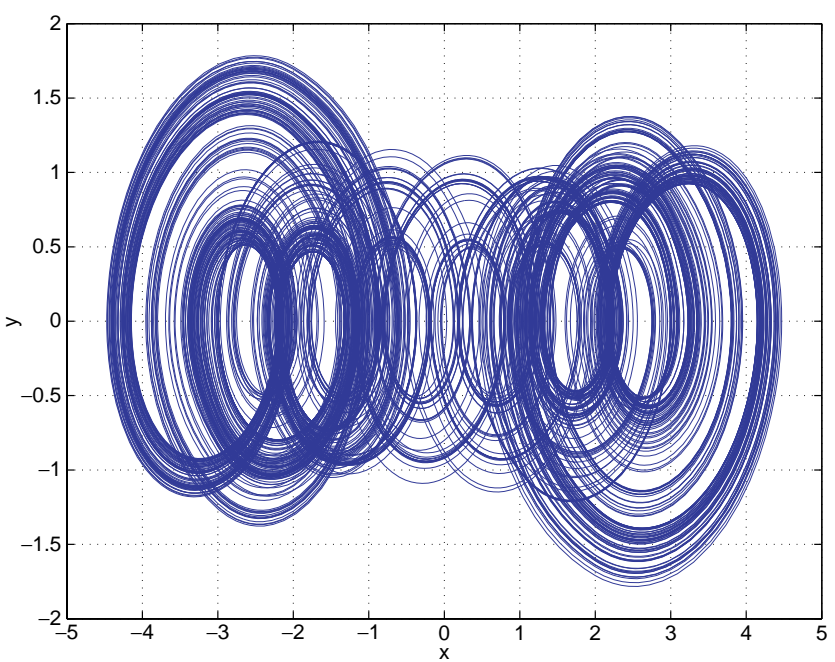

(a) 1-D 7-scroll attractor

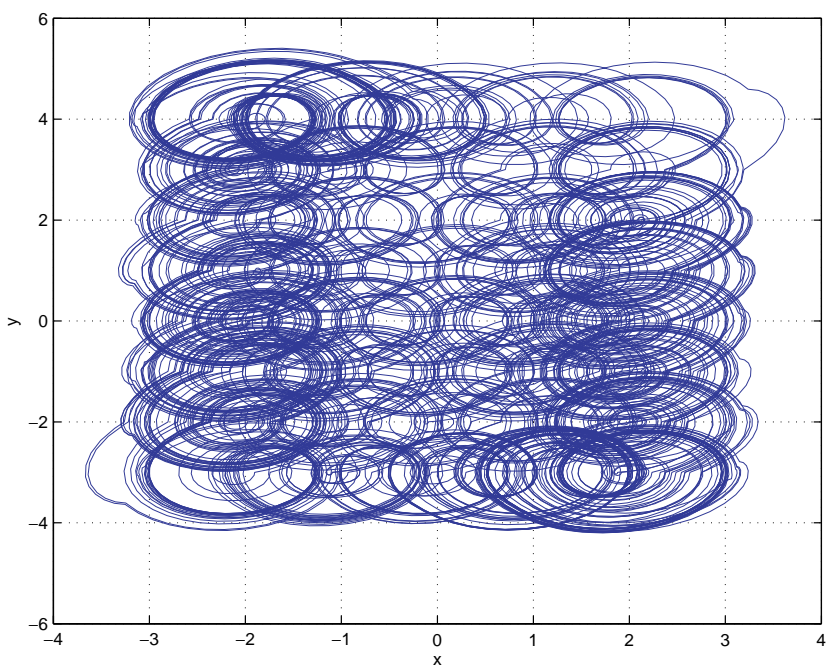

(c) 3 -D $5 \times 8 \times 3$-grid scroll attractor in $x-y$ plane

$$
\theta(\mathbf{X})=\left(\begin{array}{l}
h\left(x, p_{1}, q_{1}\right) \\
h\left(y, p_{2}, q_{2}\right) \\
h\left(z, p_{3}, q_{3}\right)
\end{array}\right),
$$

where the hysteresis series functions $h\left(x, p_{1}\right.$, $\left.q_{1}\right), h\left(y, p_{2}, q_{2}\right)$, and $h\left(z, p_{3}, q_{3}\right)$ are similarly defined by $(34)$.

Figure 41 shows the multidirectional multiscroll chaotic attractors created by the hysteresiscontrolled system (35): (a) a 1-D 7-scroll chaotic attractor, where $a=0.8, b=0.72, c=0.6$, $p=q=3$; (b) a 2-D $5 \times 7$-grid scroll chaotic attractor, where $a=0.8, b=0.7, c=0.6, p_{1}=q_{1}=2$,

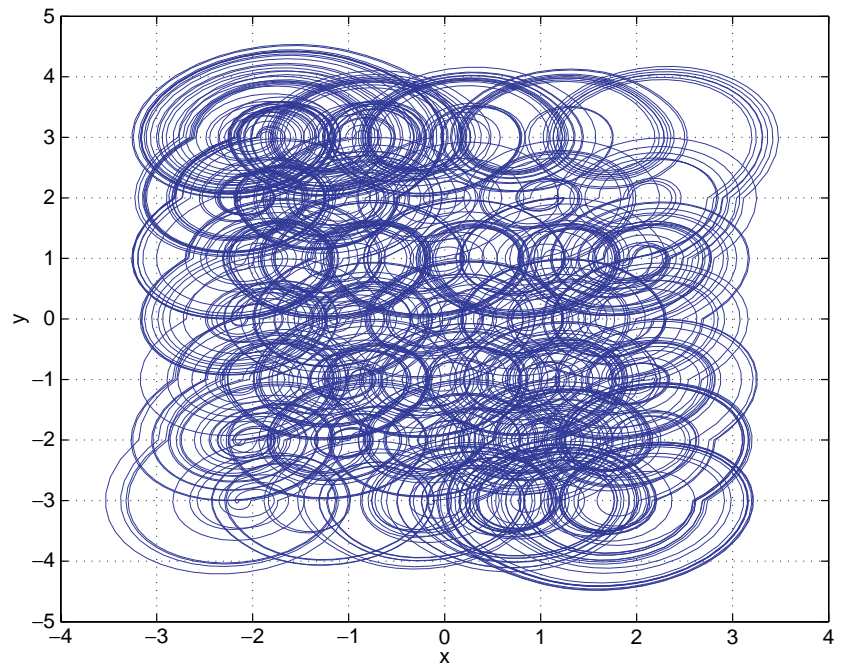

(b) 2-D $5 \times 7$-grid scroll attractor

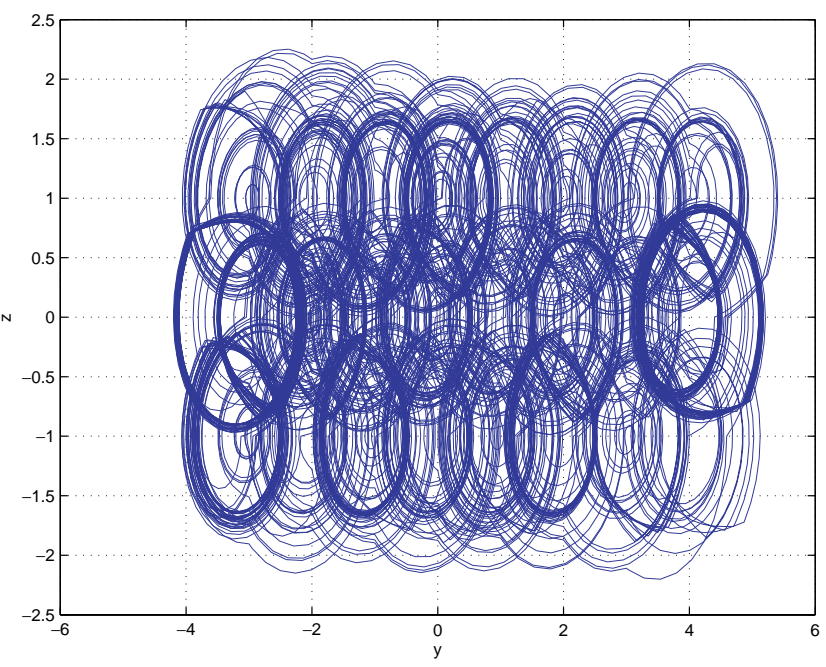

(d) 3 -D $5 \times 8 \times 3$-grid scroll attractor in $y-z$ plane

Fig. 41. Multiscroll chaotic attractors generated via hysteresis from a 3D linear autonomous system. 


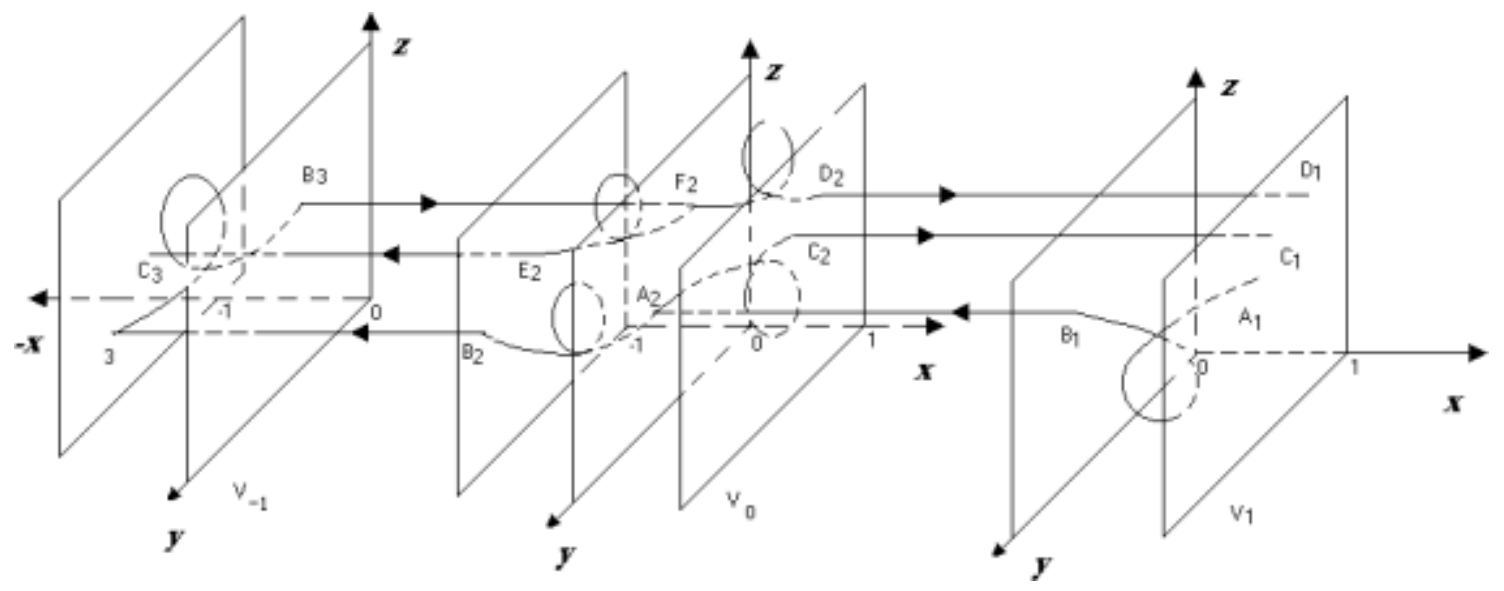

Fig. 42. Hysteresis phase space.

$p_{2}=q_{2}=3 ;$ (c), (d) a 3 -D $5 \times 8 \times 3$-grid scroll chaotic attractor, where $a=0.8, b=0.72, c=0.66$, $p_{1}=q_{1}=2, p_{2}=3, q_{2}=4, p_{3}=q_{3}=1$.

Figure 42 displays the dynamical behaviors of a 3-scroll hysteresis chaotic attractor. In particular, Lü et al. [2004d] constructed a 2D Poincaré return map to rigorously prove the chaotic behaviors of this 3 -scroll attractor. The map is described by

$$
\begin{aligned}
f: & \Omega_{1} \rightarrow \Omega_{1}, \\
& \left(y_{0}, z_{0}\right) \rightarrow\left\{\begin{array}{l}
\left(y_{2}, z_{2}\right) \quad \text { for }\left(y_{0}, z_{0}\right) \in H_{0} \\
\left(-y_{3},-z_{3}\right) \quad \text { for }\left(y_{0}, z_{0}\right) \in \bar{H}_{0},
\end{array}\right.
\end{aligned}
$$

where $\bar{H}_{0}=\Omega_{1}-H_{0}$ and $H_{0}, \Omega_{1}$ are defined in [Lü et al., $2004 \mathrm{~d}]$.

It should be pointed out that one can construct the rigorously mathematical formulation for this map by using an exact solution, as given in [Lü et al., 2004d]. However, in actual calculations, one usually applies numerical methods, such as the Newton-Raphson method, to solve the equations.

The Jacobi matrix of this map $f$ is given by

$$
D f= \begin{cases}\left(\begin{array}{ll}
\frac{\partial y_{2}}{\partial y_{0}} & \frac{\partial y_{2}}{\partial z_{0}} \\
\frac{\partial z_{2}}{\partial y_{0}} & \frac{\partial z_{2}}{\partial z_{0}}
\end{array}\right) & \text { for }\left(y_{0}, z_{0}\right) \in H_{0} \\
-\left(\begin{array}{ll}
\frac{\partial y_{3}}{\partial y_{0}} & \frac{\partial y_{3}}{\partial z_{0}} \\
\frac{\partial z_{3}}{\partial y_{0}} & \frac{\partial z_{3}}{\partial z_{0}}
\end{array}\right) & \text { for }\left(y_{0}, z_{0}\right) \in \bar{H}_{0} .\end{cases}
$$

Based on this Jacobi matrix, one can obtain exact mathematical formulations for the Lyapunov exponents $\lambda_{1}, \lambda_{2}\left(\lambda_{1} \geq \lambda_{2}\right)$ of the Poincaré map $f$. However, these formulations are rather complex. In real calculations, one usually uses numerical methods, such as that discussed in [Lü et al., 2002e], to calculate $\lambda_{1}, \lambda_{2}$. When $0<\lambda_{1}<+\infty$, the 3 -scroll hysteresis system (35) is chaotic. Similarly, one can rigorously derive some conditions for generating a 2-D $n \times m$-grid scroll and a 3-D $n \times m \times l$-grid scroll chaotic attractors via constructing a 2D Poincaré return map.

Lü et al. [2005a, 2006] also designed a novel block circuit diagram for experimentally verifying these multidirectional hysteresis multiscroll chaotic attractors. Figure 43 shows the circuit diagram, which can physically realize 1 -D $5 \sim 11$-scroll, 2 -D $3 \times 5 \sim 11$-grid scroll, and 3 -D $3 \times 3 \times 5 \sim 11$-grid scroll chaotic attractors by operating the switches.

It should be pointed out that this was the first time the experimental verification was reported for 2 -D $3 \times 11$-grid scroll and 3 -D $3 \times 3 \times 11$-grid scroll chaotic attractors. Furthermore, this design approach indicates a theoretical principle for hardware implementation of such chaotic attractors in multidirections with a large number of scrolls.

When the switches $K 1, K 2, K 3, K 4$ are switched off, the circuit shown in Fig. 43 can generate the 1-D $5 \sim 11$-scroll chaotic attractors; when the switches $K 1, K 3$ are switched on and the switches $K 2, K 4$ are switched off, this circuit can create the 2 -D $3 \times 5 \sim 11$-grid scroll chaotic attractors; when the switches $K 1, K 2, K 3, K 4$ are switched on, the circuit can generate the 3 -D $3 \times 3 \times 5 \sim 11$-grid scroll chaotic attractors. 


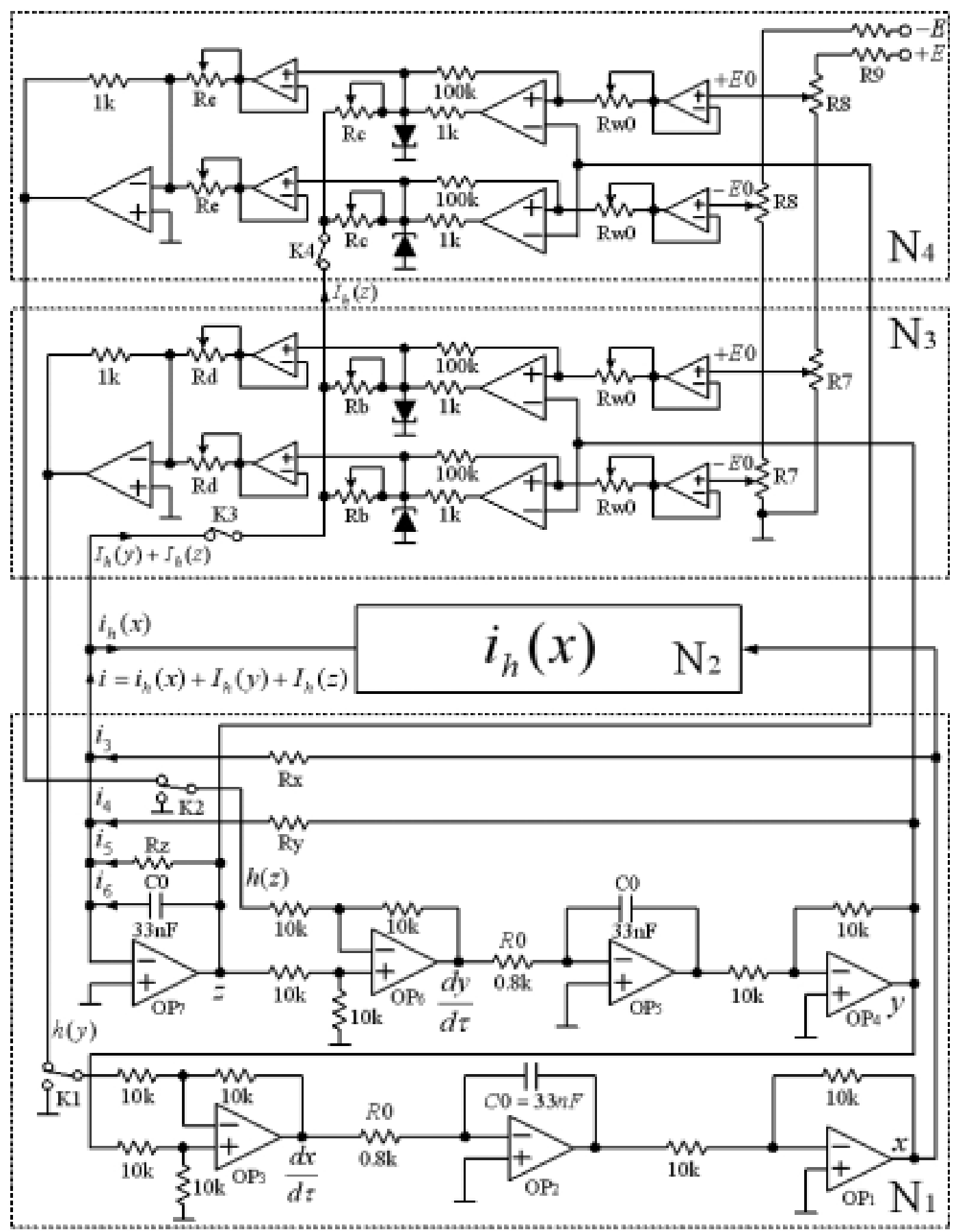

Fig. 43. Circuit diagram for realizing 3-D hysteresis multiscroll chaotic attractors.

Figure 44 displays the experimental observations of a 3 -D $3 \times 3 \times 11$-grid scroll chaotic attractor.

\section{3. $\quad$ Saturated function series approach}

This subsection briefly introduces a multidirectional saturated multiscroll chaotic system [Lü et al., 2004b, 2004c, 2005a, 2006].

Lü et al. [2004c] initiated a 3D saturated multiscroll chaotic system, described by

$$
\dot{\mathbf{X}}=\mathbf{A X}+\mathbf{B} \varphi(\mathbf{C X})
$$




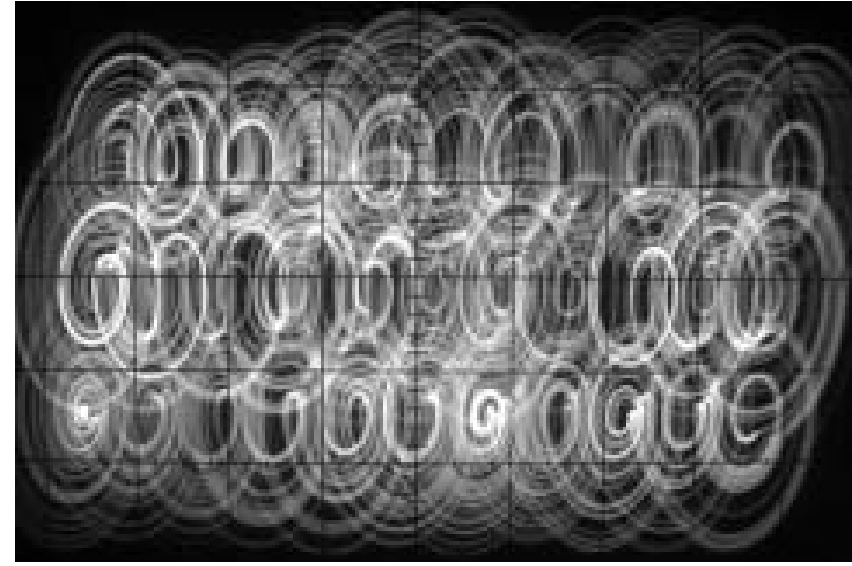

(a) $x-y$ plane, where $x=1.44 \mathrm{~V} / \operatorname{div}, y=0.8 \mathrm{~V} / \operatorname{div}$

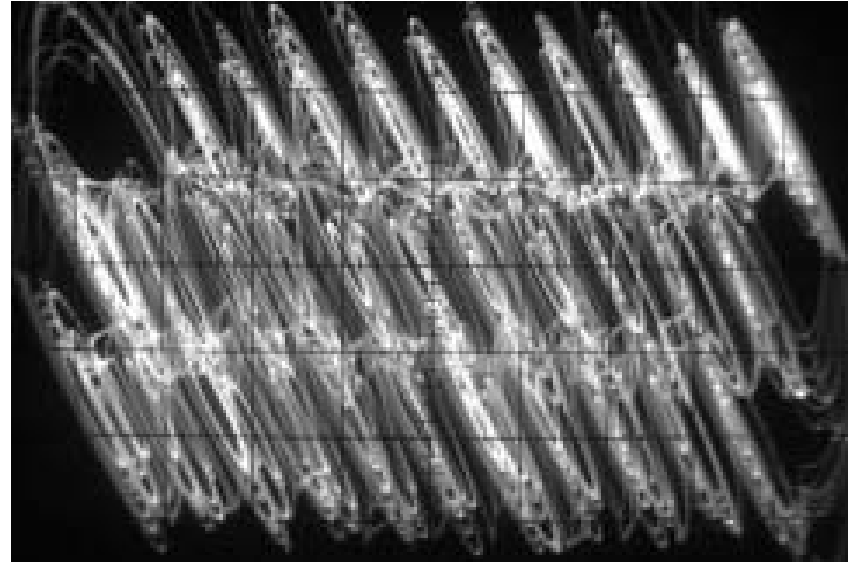

(b) $x-z$ plane, where $x=1.44 \mathrm{~V} /$ div, $z=0.8 \mathrm{~V} /$ div

Fig. 44. Experimental observations of a 3 -D $3 \times 3 \times 11$-grid scroll chaotic attractor.

where $\mathbf{X}=(x, y, z)^{T}$ is the state vector, and

$$
\begin{gathered}
\mathbf{A}=\left(\begin{array}{ccc}
0 & 1 & 0 \\
0 & 0 & 1 \\
-a & -b & -c
\end{array}\right), \quad \mathbf{B}=\left(\begin{array}{ccc}
0 & -\frac{d_{2}}{b} & 0 \\
0 & 0 & -\frac{d_{3}}{c} \\
d_{1} & d_{2} & d_{3}
\end{array}\right), \\
\mathbf{C}=\left(\begin{array}{lll}
1 & 0 & 0 \\
0 & 1 & 0 \\
0 & 0 & 1
\end{array}\right) .
\end{gathered}
$$

This system can produce three different types of attractors, as follows:

(i) 1-D saturated $n$-scroll chaotic attractors:

$$
\varphi(\mathbf{C X})=\left(\begin{array}{c}
f\left(x ; k_{1}, h_{1}, p_{1}, q_{1}\right) \\
0 \\
0
\end{array}\right),
$$

where the saturated function series $f\left(x ; k_{1}\right.$, $\left.h_{1}, p_{1}, q_{1}\right)$ is defined by

$$
\begin{aligned}
& f\left(x ; k_{1}, h_{1}, p_{1}, q_{1}\right)= \\
& \begin{cases}\left(2 q_{1}+1\right) k_{1}, & \text { if } x>q_{1} h_{1}+1 \\
k_{1}\left(x-i h_{1}\right)+2 i k_{1}, & \text { if } \begin{array}{l}
\left|x-i h_{1}\right| \leq 1 \\
-p_{1} \leq i \leq q_{1}
\end{array} \\
(2 i+1) k_{1}, & \text { if } \begin{array}{l}
1<x-i h_{1}<h_{1}-1 \\
-p_{1} \leq i \leq q_{1}-1
\end{array} \\
-\left(2 p_{1}+1\right) k_{1}, & \text { if } x<-p_{1} h_{1}-1 .\end{cases}
\end{aligned}
$$

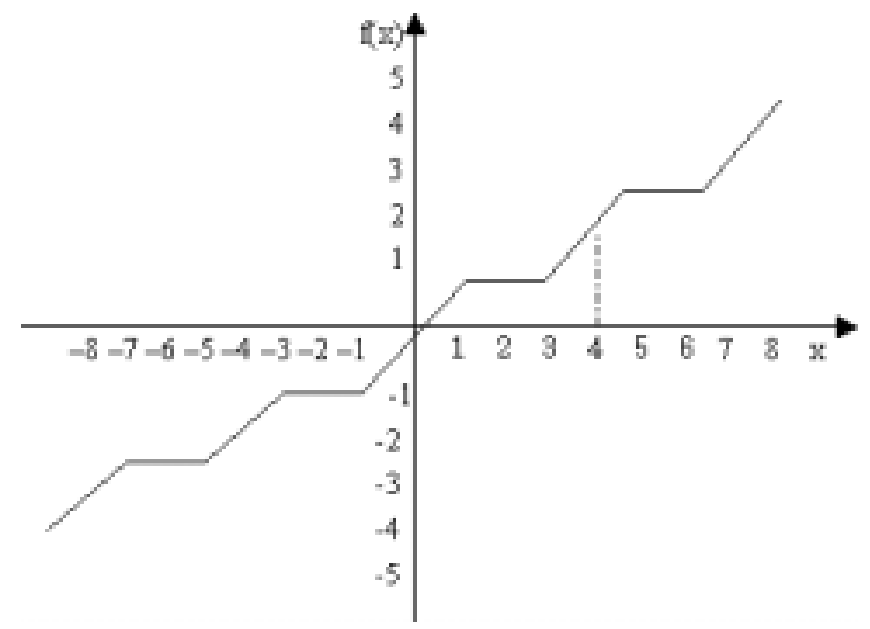

Fig. 45. Saturated function series with $k=1, h=4$.

Figure 45 displays a saturated function series with $k=1, h=4$.

(ii) 2-D saturated $n \times m$-grid scroll chaotic attractors:

$$
\varphi(\mathbf{C X})=\left(\begin{array}{c}
f\left(x ; k_{1}, h_{1}, p_{1}, q_{1}\right) \\
f\left(y ; k_{2}, h_{2}, p_{2}, q_{2}\right) \\
0
\end{array}\right),
$$

where $f\left(x ; k_{1}, h_{1}, p_{1}, q_{1}\right)$ and $f\left(y ; k_{2}, h_{2}, p_{2}\right.$, $q_{2}$ ) are similarly defined by (38).

(iii) 3 -D saturated $n \times m \times l$-grid scroll chaotic attractors:

$$
\varphi(\mathbf{C X})=\left(\begin{array}{l}
f\left(x ; k_{1}, h_{1}, p_{1}, q_{1}\right) \\
f\left(y ; k_{2}, h_{2}, p_{2}, q_{2}\right) \\
f\left(z ; k_{3}, h_{3}, p_{3}, q_{3}\right)
\end{array}\right),
$$


where $f\left(x ; k_{1}, h_{1}, p_{1}, q_{1}\right), \quad f\left(y ; k_{2}, h_{2}, p_{2}, q_{2}\right)$, and $f\left(z ; k_{3}, h_{3}, p_{3}, q_{3}\right)$ are similarly defined by $(38)$.

When $a=b=c=d_{1}=0.7, k_{1}=9, h_{1}=18$, $p_{1}=2, q_{1}=2$, system $(37)$ has a 1 -D 6 -scroll chaotic attractor, as shown in Fig. 46(a); when $a=b=c=d_{1}=d_{2}=0.7, k_{1}=k_{2}=50$, $h_{1}=h_{2}=100, p_{1}=q_{1}=p_{2}=q_{2}=2$, system (37) has a 2-D $6 \times 6$-grid scroll chaotic attractor, as shown in Fig. 46(b); when $a=d_{1}=0.7, b=c$ $=d_{2}=d_{3}=0.8, k_{1}=100, h_{1}=200, k_{2}=k_{3}=40$, $h_{2}=h_{3}=80, p_{1}=p_{2}=p_{3}=q_{1}=q_{2}=q_{3}=2$, system (37) has a 3-D $6 \times 6 \times 6$-grid scroll chaotic attractor, as shown in Figs. 46(b) and 46(c).

Moreover, Lü et al. [2004c] also constructed a 2D Poincaré return map to rigorously prove the chaotic behaviors of such saturated double-scroll attractors. The map is described by

$$
\left(y_{0}, z_{0}\right) \rightarrow \begin{cases}\left(y_{1}, z_{1}\right) & \text { for }\left(y_{0}, z_{0}\right) \in M_{1}-M_{1}^{-} \\ \left(y_{2}, z_{2}\right) & \text { for }\left(y_{0}, z_{0}\right) \in \Gamma \\ \left(-y_{3},-z_{3}\right) & \text { for }\left(y_{0}, z_{0}\right) \in M_{1}^{-}-\Gamma,\end{cases}
$$

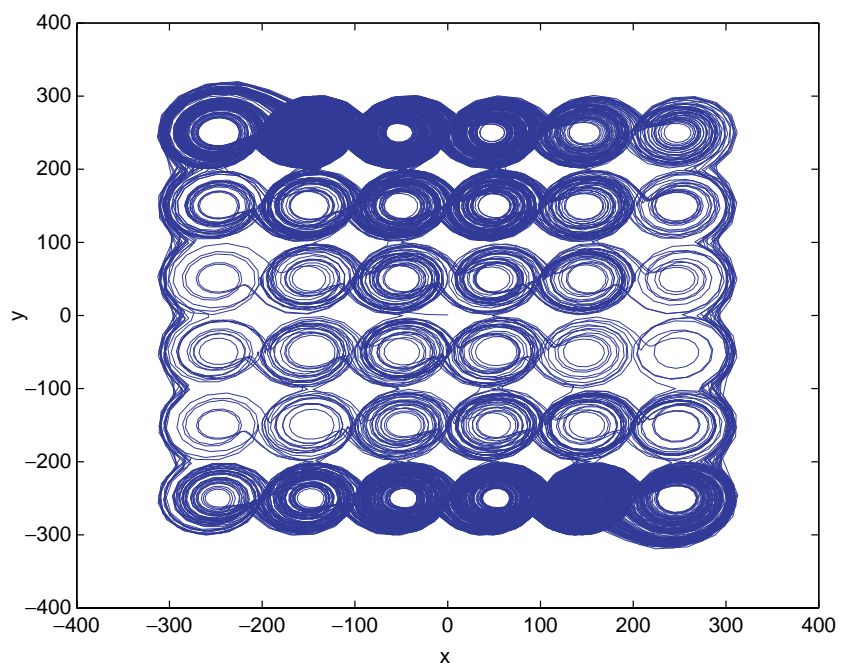

(b) 2 -D $6 \times 6$-grid scroll attractor

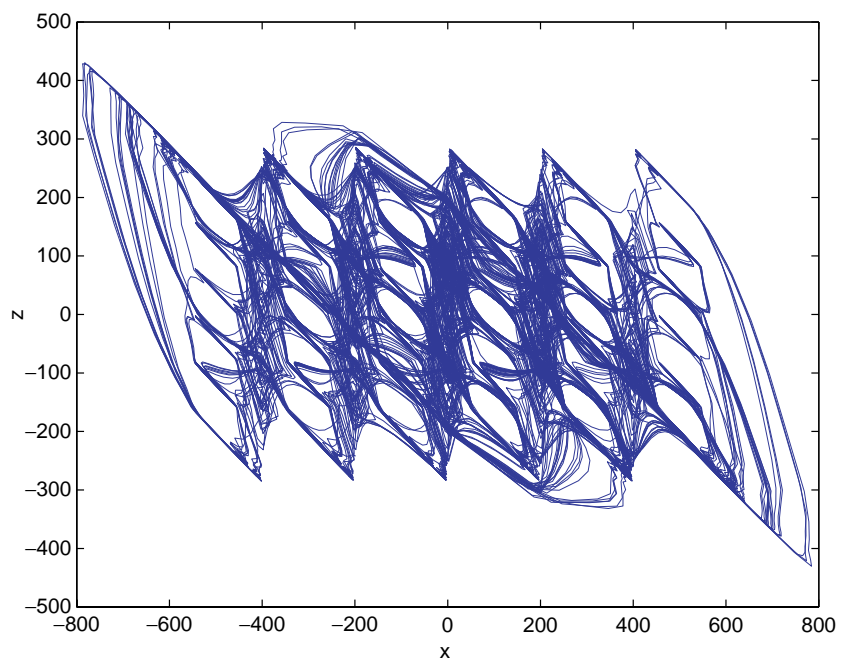

(d) 3 -D $6 \times 6 \times 6$-grid scroll attractor in the $x-z$ plane

(c) 3 -D $6 \times 6 \times 6$-grid scroll attractor in the $x-y$ plane

Fig. 46. Numerical simulations for saturated multiscroll chaotic attractors. 
where $M_{1}, M_{1}^{-}, \Gamma$ are defined in [Lü et al., 2004c]. Notice that one can deduce a rigorously mathematical formulation for this map by using the exact solutions given in [Lü et al., 2004c].

The Jacobi matrix of this map $F$ is given by

$$
D F= \begin{cases}\left(\begin{array}{ll}
\frac{\partial y_{1}}{\partial y_{0}} & \frac{\partial y_{1}}{\partial z_{0}} \\
\frac{\partial z_{1}}{\partial y_{0}} & \frac{\partial z_{1}}{\partial z_{0}}
\end{array}\right) & \text { for }\left(y_{0}, z_{0}\right) \in M_{1}-M_{1}^{-} \\
\left(\begin{array}{ll}
\frac{\partial y_{2}}{\partial y_{0}} & \frac{\partial y_{2}}{\partial z_{0}} \\
\frac{\partial z_{2}}{\partial y_{0}} & \frac{\partial z_{2}}{\partial z_{0}}
\end{array}\right) & \text { for }\left(y_{0}, z_{0}\right) \in \Gamma \\
-\left(\begin{array}{ll}
\frac{\partial y_{3}}{\partial y_{0}} & \frac{\partial y_{3}}{\partial z_{0}} \\
\frac{\partial z_{3}}{\partial y_{0}} & \frac{\partial z_{3}}{\partial z_{0}}
\end{array}\right) & \text { for }\left(y_{0}, z_{0}\right) \in M_{1}^{-}-\Gamma\end{cases}
$$

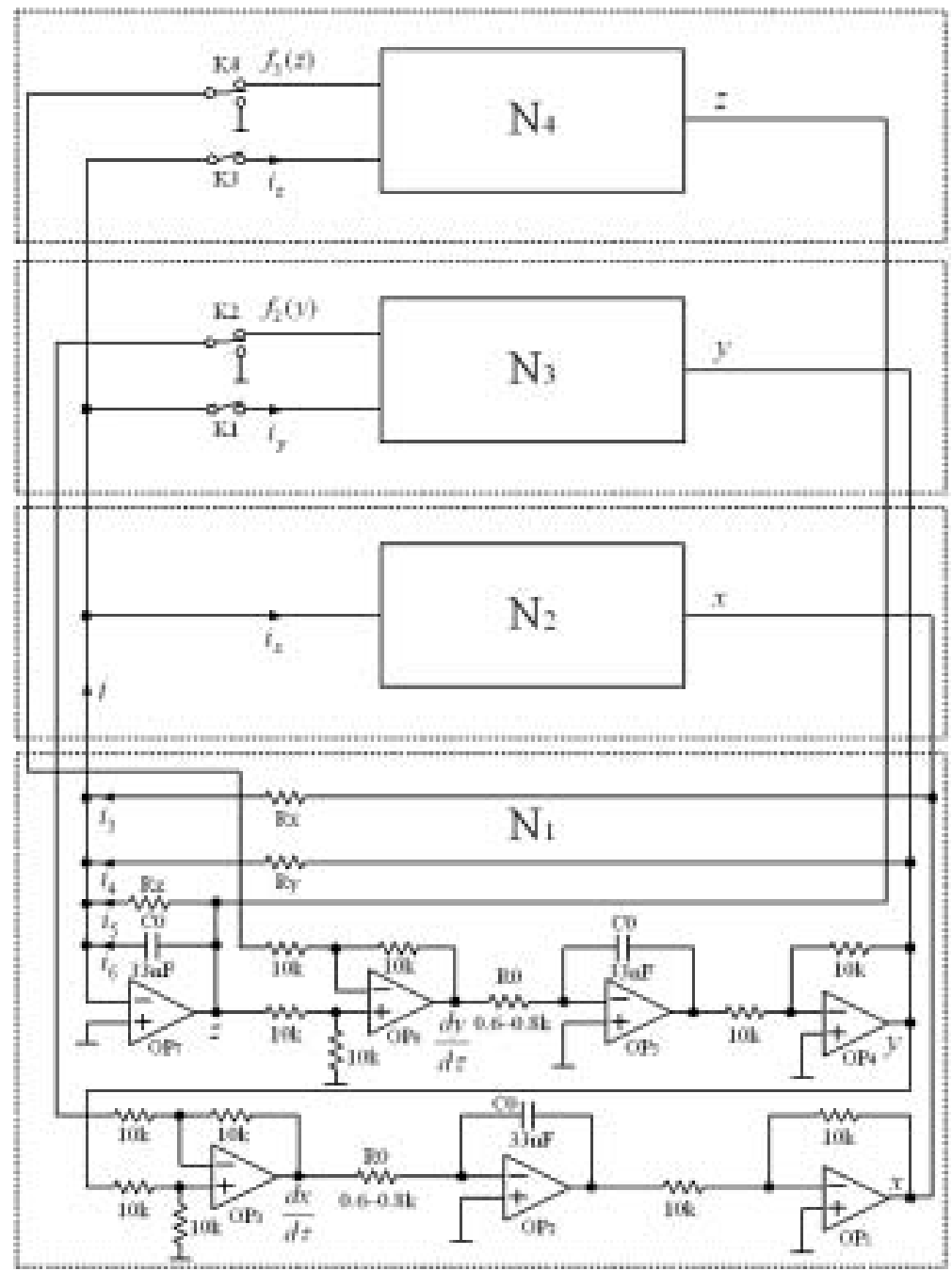

Fig. 47. Block circuitry for realizing multidirectional saturated multiscroll chaotic attractors. 
Thus, one can obtain exact mathematical expressions for the Lyapunov exponents $L E_{1}, L E_{2}$ of the mapping $F$ from the Jacobian of $F$. However, these formulations are rather complex. In real calculations, one often uses numerical methods, as described in [Lü et al., 2002e], to calculate the maximum Lyapunov exponent $L E_{1}$. If $0<L E_{1}<+\infty$, system (37) has a chaotic attractor. Furthermore, one can also rigorously prove the chaotic behaviors of the attractor by using the Homoclinic type of Šilnikov Theorem [Chen \& Lü, 2003; Silva, 1993].

More recently, Lü et al. [2006] designed a novel block circuit diagram for generating multidirectional saturated multiscroll chaotic attractors, as

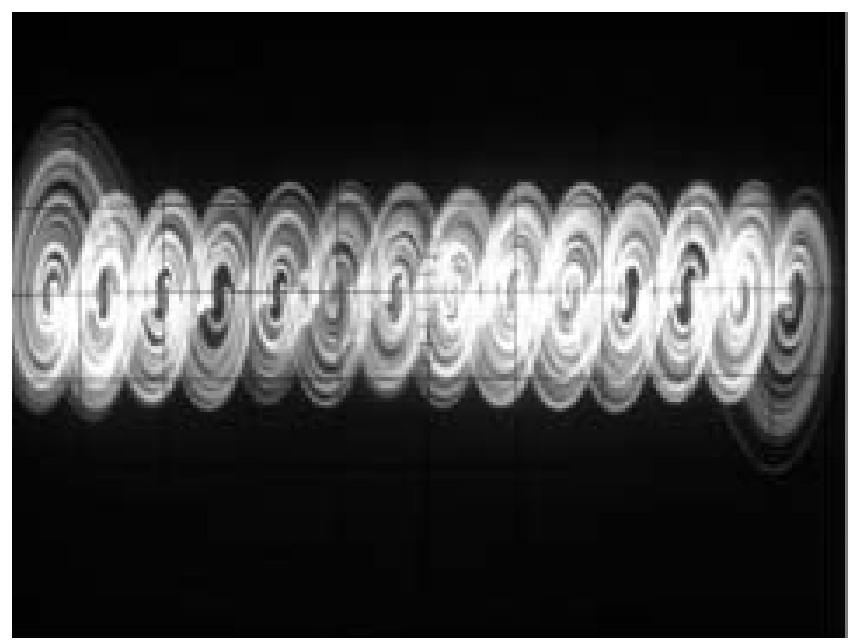

(a) 1-D 14-scroll attractor in the $x-y$ plane, where $x=1.4 \mathrm{~V} / \operatorname{div}, y=0.4 \mathrm{~V} / \operatorname{div}$

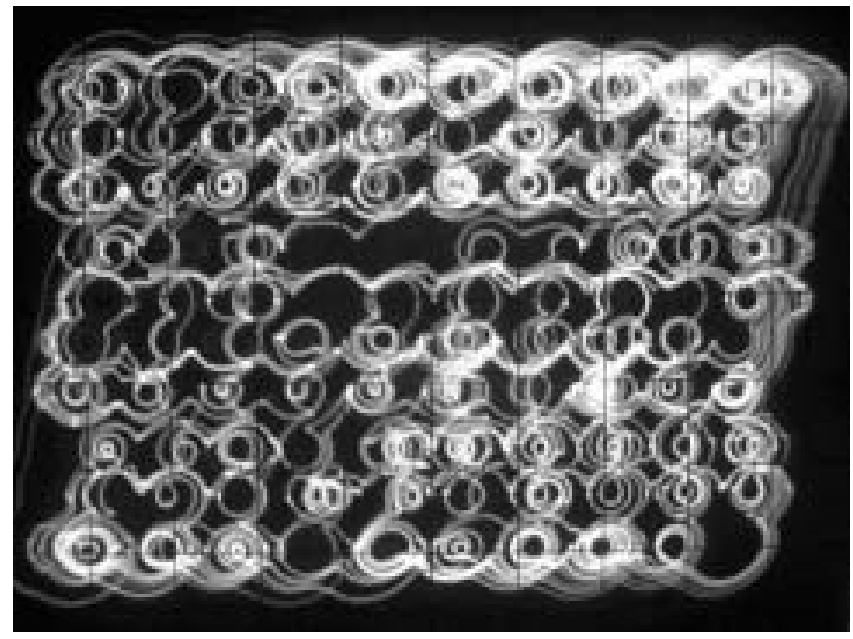

(c) 3 -D $10 \times 10 \times 10$-grid scroll attractor in the $x-y$ plane, where $x=1.0 \mathrm{~V} / \operatorname{div}, y=0.8 \mathrm{~V} / \mathrm{div}$ shown in Fig. 47. The circuit consists of five different function parts: $N 1, N 2, N 3, N 4$, and the switch sets. Here, $N 1$ includes three integrators, two inverter-amplifiers and two subtractor amplifiers; $N 2$ consists of the generator for the $x$-directional current saturated function series $i_{x}(x)$ and the generator for the $x$-directional voltage saturated function series $f_{1}(x) ; N 3$ includes the generator for the $y$-directional current saturated function series $i_{y}(y)$ and the generator for the $y$-directional voltage saturated function series $f_{2}(y) ; N 4$ consists of the generator for the $z$-directional current saturated function series $i_{z}(z)$ and the generator for the $z$-directional voltage saturated function series $f_{3}(z)$.

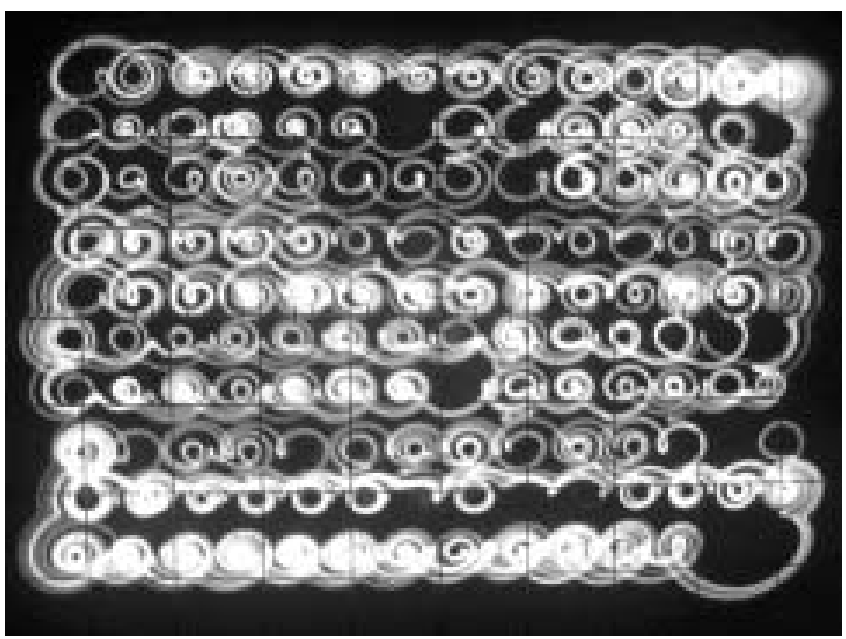

(b) 2 -D $14 \times 10$-grid scroll attractor in the $x-y$ plane, where $x=1.4 \mathrm{~V} / \operatorname{div}, y=1.6 \mathrm{~V} / \operatorname{div}$

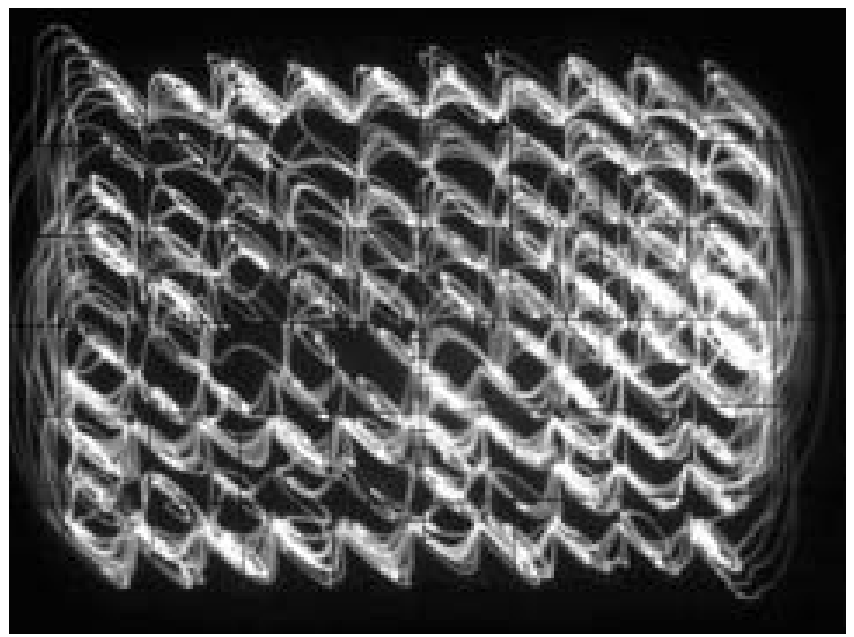

(d) 3 -D $10 \times 10 \times 10$-grid scroll attractor in the $x-z$ plane, where $x=1.0 \mathrm{~V} / \operatorname{div}, z=0.8 \mathrm{~V} / \operatorname{div}$

Fig. 48. Experimental observations of saturated multiscroll chaotic attractors. 
All required circuit parameters are given in [Lü et al., 2006] for generating various multiscroll chaotic attractors. Figure 48 displays the experimental observations of some saturated multiscroll chaotic attractors.

The designed block circuitry can physically realize 1-D 10,12,14-scroll, 2-D 10,12, $14 \times 10$-grid scroll, and 3 -D $10 \times 10 \times 10$-grid scroll chaotic attractors via operating the switches. Particularly, it was the first time in the literature to report the experimental verification of 1 -D 14-scroll, 2 -D $14 \times 10$-grid scroll and 3 -D $10 \times 10 \times 10$-grid (1000) scroll chaotic attractors. It should be pointed out that hardware implementation of 1 -D $n$-scroll with $n \geq 10$, 2-D $n \times m$-grid scroll with $n, m \geq 10$, and 3 -D $n \times m$ $\times l$-grid scroll with $n, m, l \geq 10$ chaotic attractors is very difficult technically. The above circuit design method provides a theoretical principle for hardware implementation of such chaotic attractors with multidirectional orientations and a large number of scrolls.

\section{Design of Multiscroll Chaotic Attractors via Switching Manifolds}

This section introduces several basic approaches for generating multiscroll chaotic attractors from some simple linear systems by using switching-manifold control.

\subsection{Design of chaotic attractors with multiple merged basins of attraction via switching control}

This subsection presents a switching control method for creating $n$-scroll chaotic attractors from a $3 \mathrm{D}$ linear autonomous system [Lü, 2003; Lü et al., 2002a, 2002b, 2002f, 2003a, 2004b].

Lü et al. [2002f; 2003a] proposed a systematic switching PWL function control approach for generating chaotic attractors with multiple merged basins of attraction from the following 3D linear controlled system:

$$
\dot{\mathbf{X}}=\mathbf{A X}+\mathbf{U}(\mathbf{X})
$$

where $\mathbf{X}=(x, y, z)^{T}$,

$$
\mathbf{A}=\left(\begin{array}{ccc}
a & b & 0 \\
-b & a & 0 \\
0 & 0 & c
\end{array}\right)
$$

and

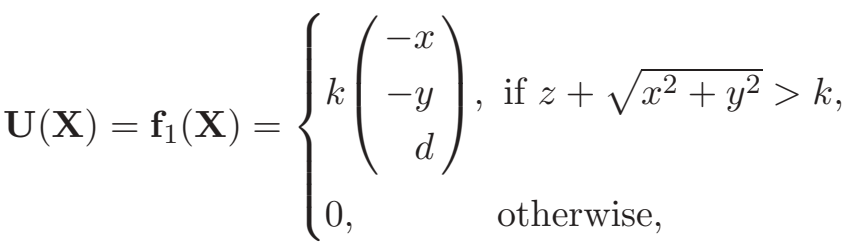

in which $a, b, c, d, k$ are real parameters. System (40) with controller $\mathbf{U}(\mathbf{X})=\mathbf{f}_{1}(\mathbf{X})$ can create chaotic attractors within a wide range of parameter values.

To generate two chaotic attractors simultaneously from system (40), the controller $\mathbf{U}(\mathbf{X})$ is modified as follows:

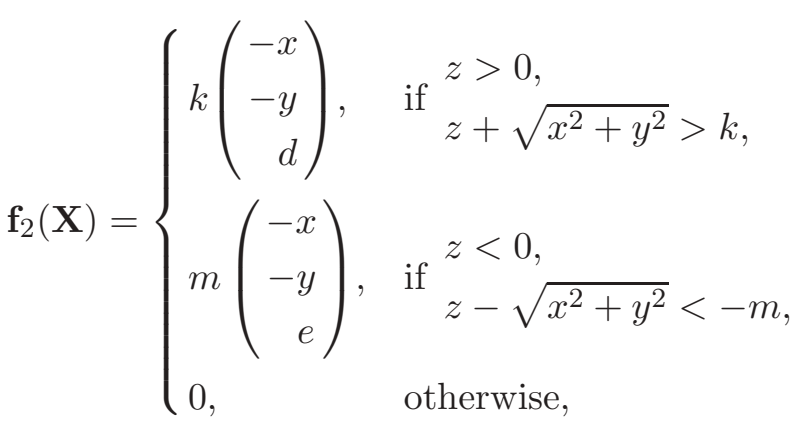

where $a, b, c, d, e, k, m$ are real parameters.

Furthermore, system (40) with controller $\mathbf{U}(\mathbf{X})=\mathbf{f}_{2}(\mathbf{X})$ can simultaneously create two chaotic attractors, an upper-attractor and a lowerattractor, within a wide range of parameter values. Notice that $z=0$ is the invariant manifold of system (40) with controller $\mathbf{U}(\mathbf{X})=\mathbf{f}_{2}(\mathbf{X})$. When $a=3, b=20, c=-20, d=10, e=-10, k=4$, $m=4$, system (40) with controller $\mathbf{U}(\mathbf{X})=\mathbf{f}_{2}(\mathbf{X})$ has an upper and a lower chaotic attractors simultaneously, as shown in Fig. 49.

Remark 3. In a similar way, one can easily construct $n$ different chaotic attractors simultaneously with different initial values in the switching system (40) via parallel displacement and rotation transformations. Since all the chaotic attractors are bounded by a finite sphere, one can partition the whole space into $n$ disjoint subspaces, and then duplicate the original attractor - the upper-attractor or the lower-attractor - into every subspace. It should be pointed out that the above $n$ attractors are independent of one another; that is, there is no system orbit that connects any two different attractors together.

When $n=2$, to connect together the orbits of the upper- and lower-attractors, so as to obtain a single chaotic attractor, one can design a controller 


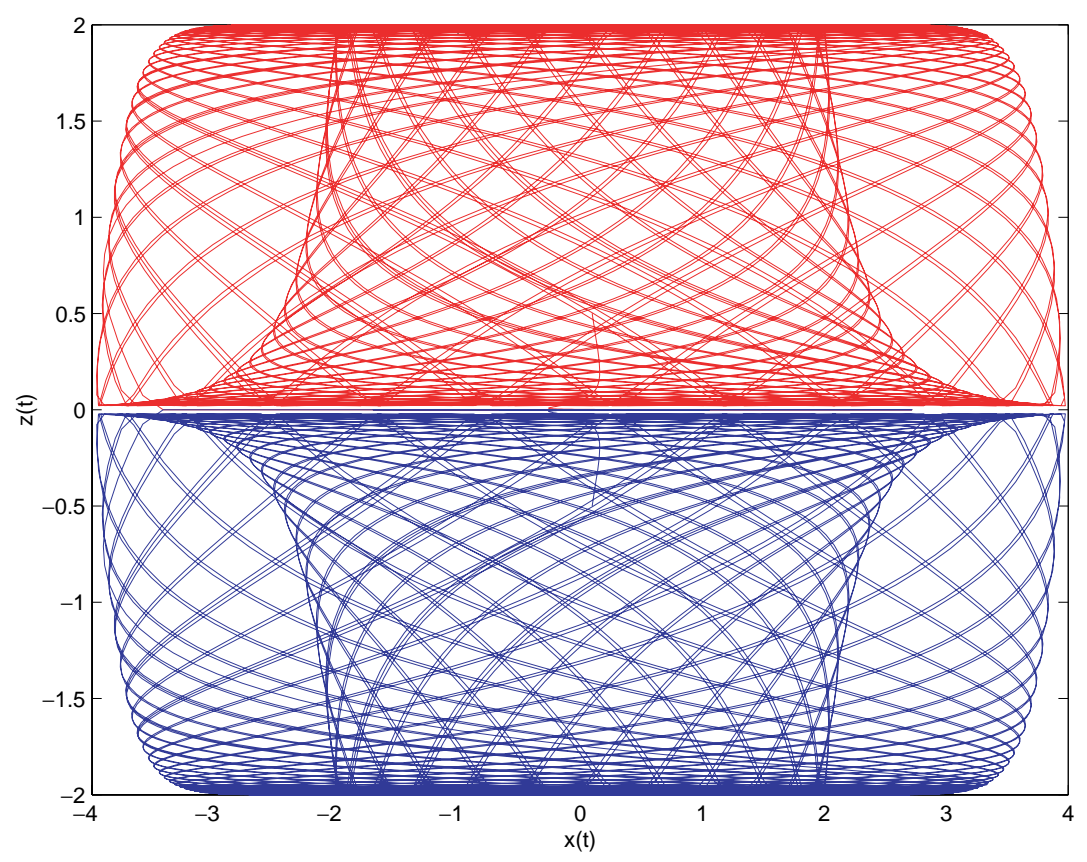

Fig. 49. Upper-attractor and lower-attractor.

to force the orbit of the upper-attractor to go through the invariant manifold $z=0$ from above and then enter into the subspace $\{(x, y, z) \mid z<0\}$. At the same time, this controller should have the function to force the orbit of the lower-attractor to go through the invariant manifold $z=0$ from below and then return to the subspace $\{(x, y, z) \mid z>0\}$. Based on this idea, one uses $\delta(0,0,-\operatorname{sign}(z))^{\top}$ to substitute for 0 in the controller $\mathbf{f}_{2}(\mathbf{X})$, thereby yielding the modified controller as follows:

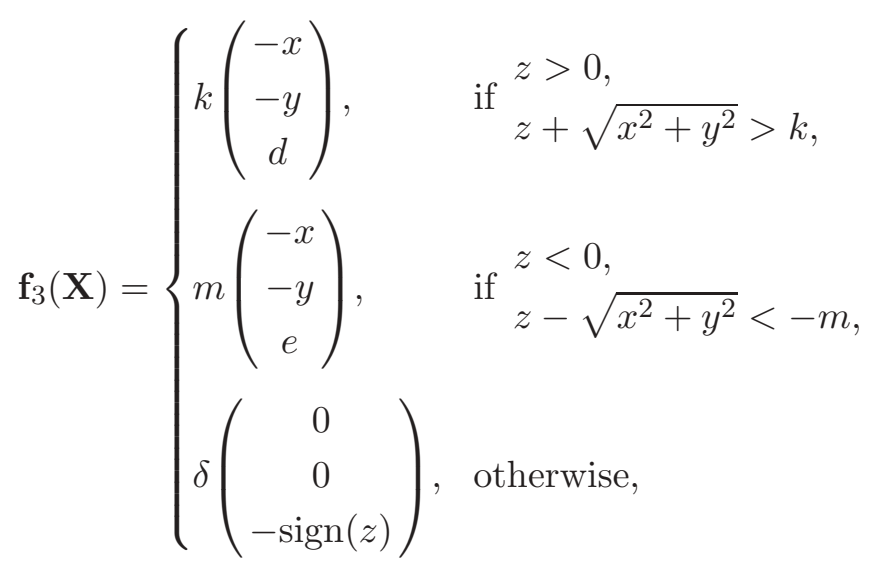

where $a, b, c, d, e, k, m, \delta$ are all real parameters.

When $a=3, b=20, c=-20, d=-e=10$, $k=m=4, \delta=1$, system (40) with controller $\mathbf{U}(\mathbf{X})=\mathbf{f}_{3}(\mathbf{X})$ has a chaotic attractor with two merged basins of attraction, the upper basin of attraction and the lower basin of attraction, as shown in Fig. 50(a). Notice that the controller $\mathbf{U}(\mathbf{X})=\mathbf{f}_{3}(\mathbf{X})$ has three switching planes: $S_{1}, S_{2}$, and $z=0$, in which the two switching planes $S_{1}$ and $S_{2}$ are responsible for the generation of two chaotic attractors, the upper chaotic attractor and the lower chaotic attractor; while the switching plane $z=0$ is responsible for the connection of these two chaotic attractors.

Furthermore, to generate a chaotic attractor with three merged basins of attraction, by using $\delta(0,0,-\operatorname{sign}(z-h)-c h / \delta)^{\top}$, one can construct the controller $\mathbf{U}(\mathbf{X})$ as follows: 


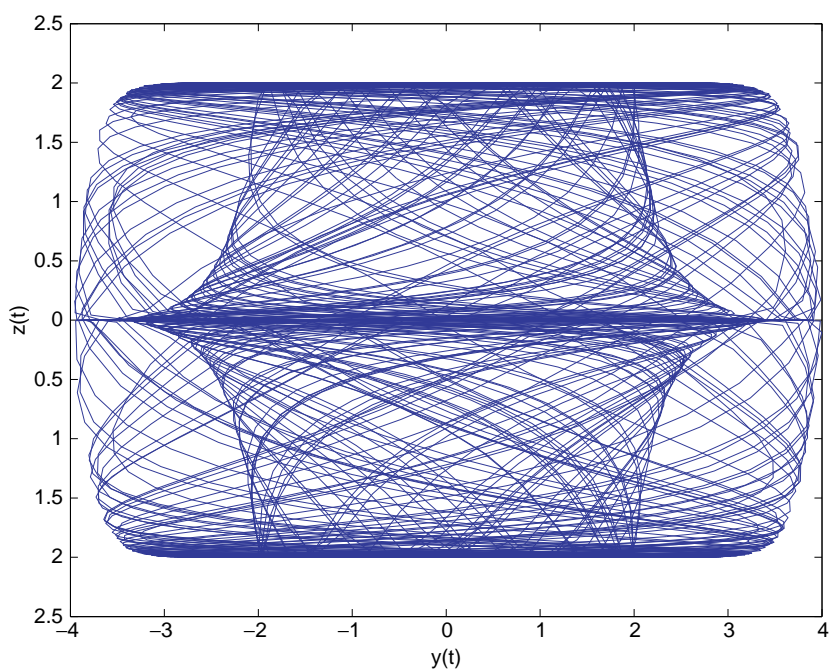

(a) Attractor with two merged basins of attraction

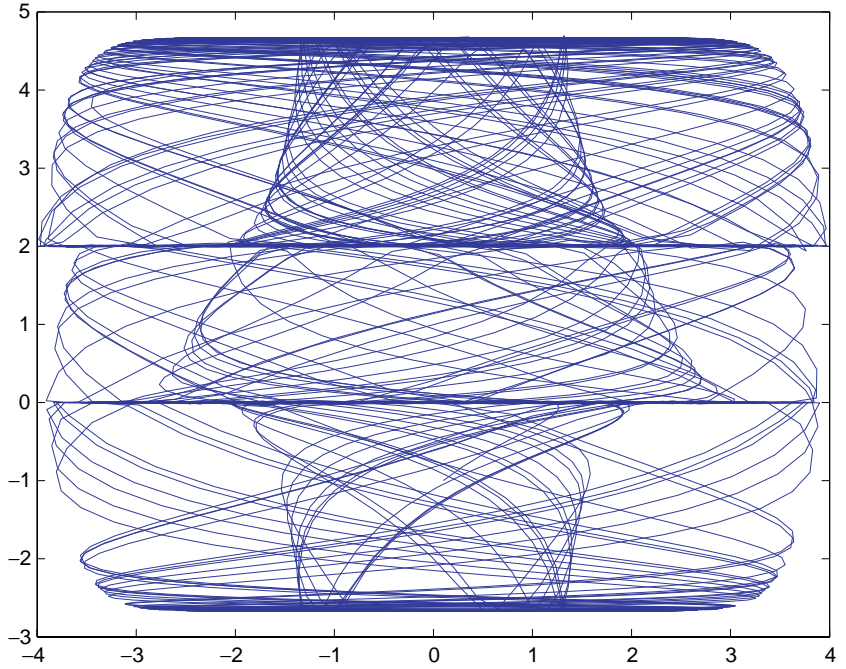

(b) Attractor with three merged basins of attraction

Fig. 50. Chaotic attractors with multiple merged basins of attraction in the $y-z$ plane.

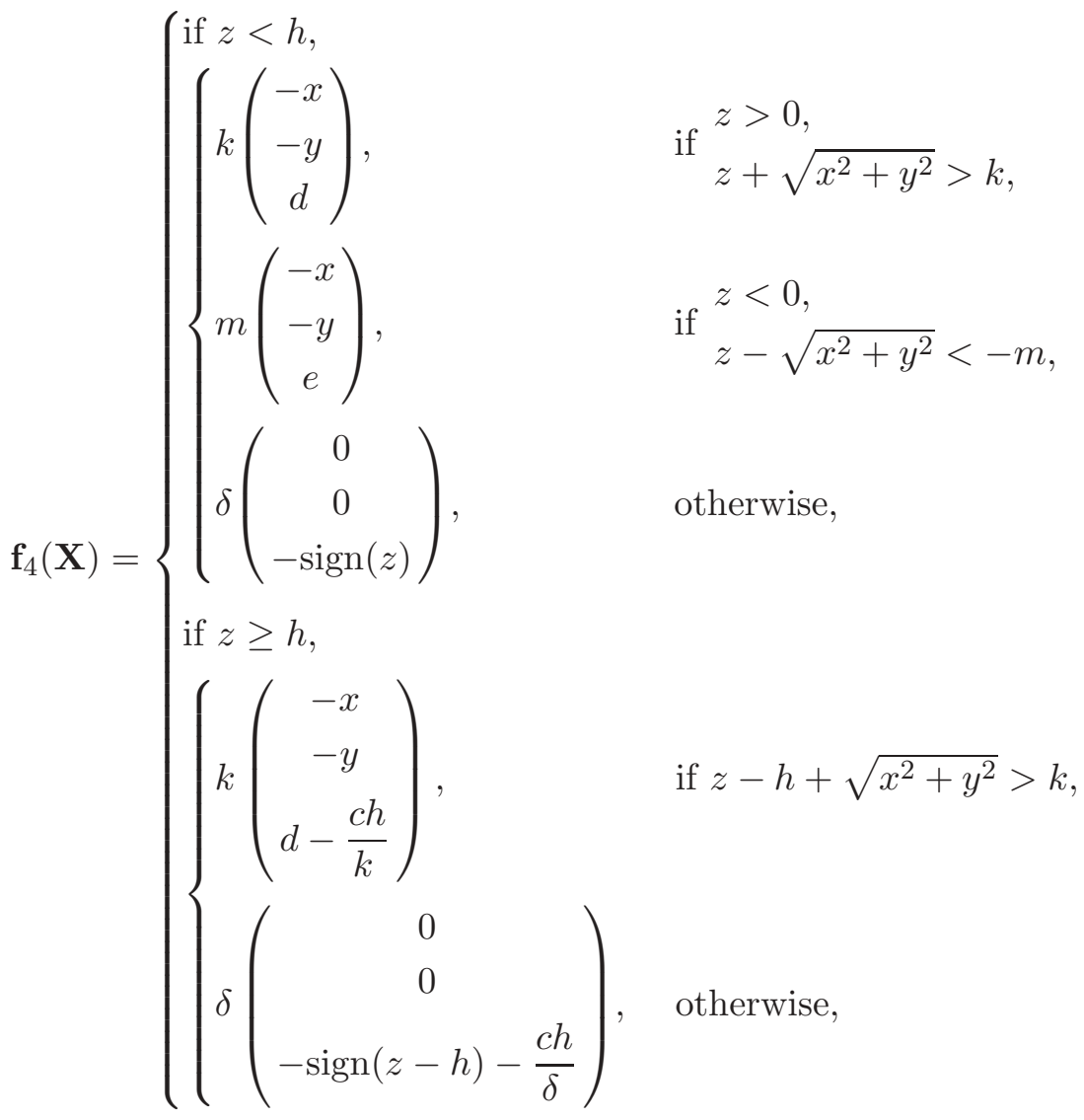

where $a, b, c, d, e, k, m, h, \delta$ are all real parameters.

When $a=3, b=20, c=-15, d=-e=$ $10, k=m=4, h=2, \delta=5$, system (40) with controller $\mathbf{U}(\mathbf{X})=\mathbf{f}_{4}(\mathbf{X})$ has a chaotic attractor with three merged basins of attraction: two upper basins of attraction and one lower basin of attraction, as shown in Fig. 50(b).

Similarly, one can generate chaotic attractors with $n$ merged basins of attraction. The formalized 
design approach is outlined as follows:

(1) Partition the whole space into $n$ subspaces. For convenience, one may partition the space along the $z$-axis.

(2) Duplicate the original attractors, the upperattractor and the lower-attractor, to every subspace.

(3) Use the switching controller to connect all the $n$ independent attractors, so as to form a single chaotic attractor with multiple merged basins of attraction, as depicted by Fig. 51 .

Here, the switching controller can be chosen as $\delta \operatorname{sign}\left(z-h_{i}\right)$, where the height $h_{i}$ (between two neighboring subspaces) should be smaller than the height of a single chaotic attractor.

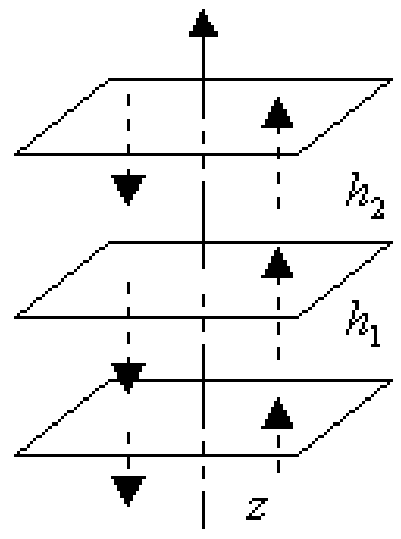

Fig. 51. Illustrative sketch for the connection of orbit.

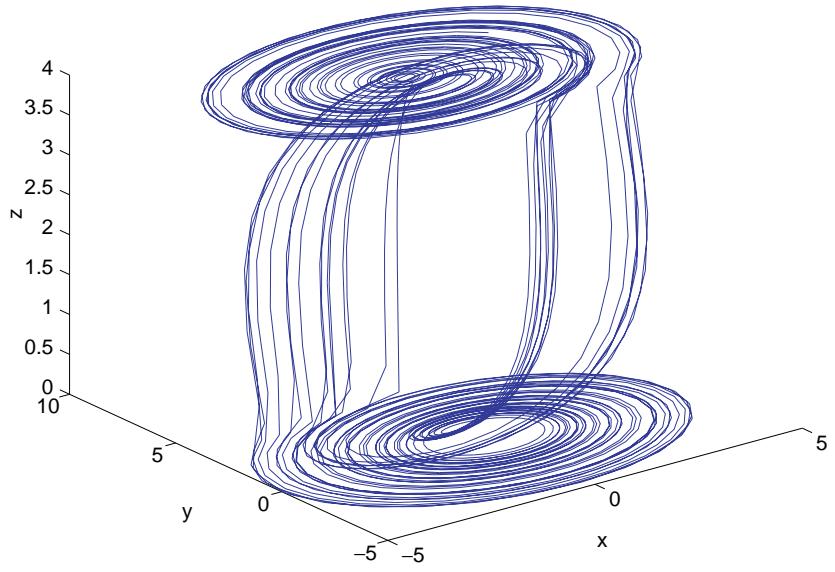

(a) Double-scroll chaotic attractor

\subsection{Design of multiscroll chaotic attractors via step series switching}

This subsection briefly introduces the step series switching approach for generating multiscroll chaotic attractors from some simple linear systems.

Yang and Li [2003] proposed the following PWL function switching system:

$$
\dot{\mathbf{X}}=\mathbf{A}\left[\mathbf{X}-s\left(\mathbf{C}^{T} \mathbf{X}-4\right) \mathbf{B}\right],
$$

where

$$
s(\eta)= \begin{cases}1 & \eta>0 \\ 0 & \eta \leq 0\end{cases}
$$

and

$$
\mathbf{A}=\left(\begin{array}{ccc}
0.5 & 10 & 0 \\
-10 & 0.5 & 0 \\
0 & 0 & -10
\end{array}\right), \mathbf{B}=\left(\begin{array}{l}
b \\
4 \\
4
\end{array}\right), \mathbf{C}=\left(\begin{array}{l}
0 \\
1 \\
1
\end{array}\right)
$$

When $b=0$, system (41) has a double-scroll chaotic attractor, as shown in Fig. 52(a).

To create $n$-scroll chaotic attractors, Yang and $\mathrm{Li}$ [2003] modified the function $s(\eta)$ defined in (42) as follows:

$$
s(\eta)= \begin{cases}h_{1} & \eta \leq a_{1} \\ h_{2} & a_{i-1}<\eta \leq a_{i} \quad \text { for } 2 \leq i \leq n-1 \\ h_{n} & \eta>a_{n},\end{cases}
$$

where $\left\{a_{1}, \ldots, a_{n}\right\}$ and $\left\{h_{1}, \ldots, h_{n}\right\}$ are strictly increasing constant series. Thus, system (41) becomes

$$
\dot{\mathbf{X}}=\mathbf{A}\left[\mathbf{X}-s\left(\mathbf{C}^{T} \mathbf{X}\right) \mathbf{B}\right],
$$

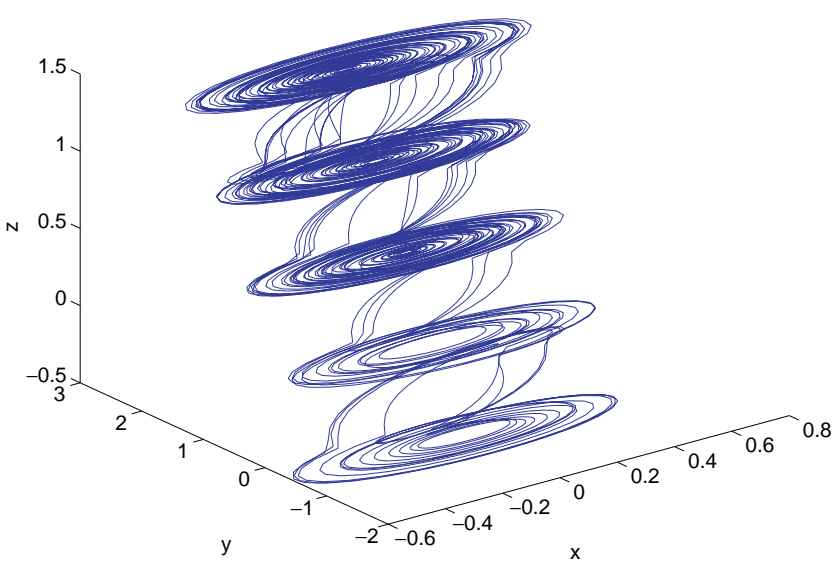

(b) 5-Scroll chaotic attractor

Fig. 52. $n$-Scroll chaotic attractors generated via step series switching. 
where

$\mathbf{A}=\left(\begin{array}{ccc}\alpha & \beta & 0 \\ -\beta & \alpha & 0 \\ 0 & 0 & -\gamma\end{array}\right), \quad \mathbf{B}=\left(\begin{array}{l}0 \\ d \\ d\end{array}\right), \quad \mathbf{C}=\left(\begin{array}{l}0 \\ 1 \\ 1\end{array}\right)$.

When $a_{1}=-0.5, a_{2}=0.5, a_{3}=1.5, a_{4}=2.5$, $h_{1}=-1, h_{2}=0, h_{3}=1, h_{4}=2, h_{5}=3, \alpha=0.5$, $\beta=10, \gamma=5, d=0.5$, system (44) has a 5-scroll chaotic attractor, as shown in Fig. 52(b).

Recently, Li et al. [2003] constructed a circuit diagram, as shown in Fig. 53, for generating multiscroll chaotic attractors. The circuit equation is described by

$$
\left(\begin{array}{c}
\dot{v}_{1} \\
\dot{v}_{2} \\
\dot{v}_{3}
\end{array}\right)=\frac{1}{C}\left(\begin{array}{ccc}
0 & \frac{1}{R_{f 21}} & 0 \\
-\frac{1}{R_{f 12}} & \frac{1}{R_{f 22}} & 0 \\
0 & 0 & -\frac{1}{R_{f 33}}
\end{array}\right),
$$
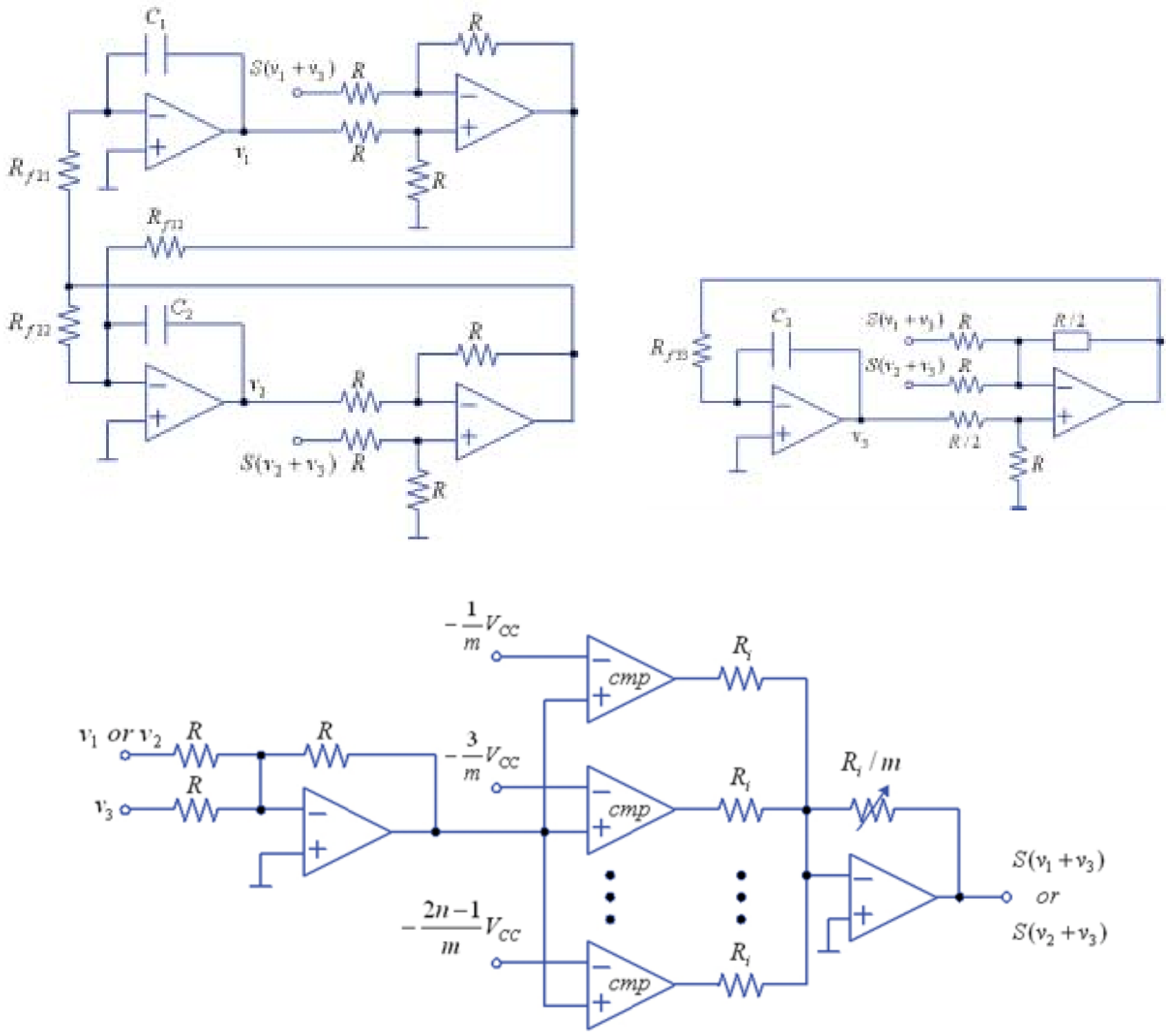

Fig. 53. Circuit diagram for 7-scroll chaotic attractors. 


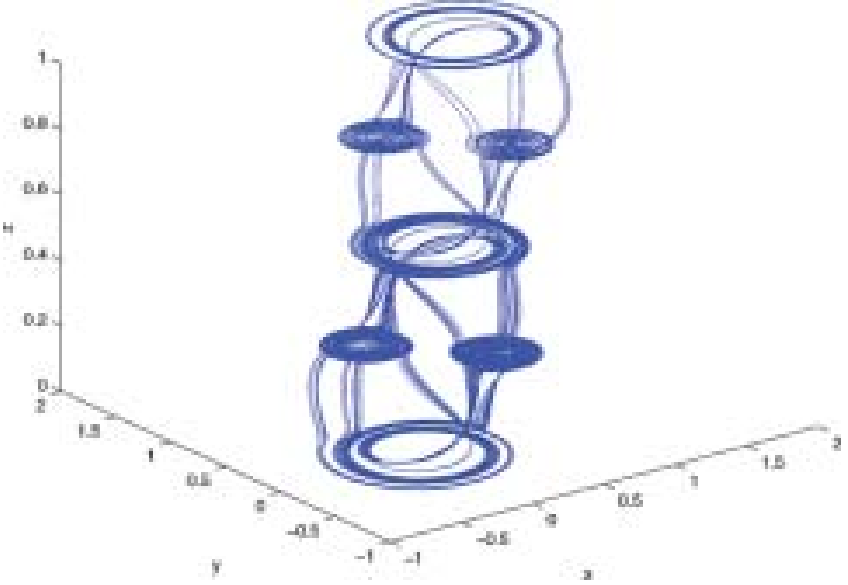

(a) 7-Scroll chaotic attractor obtained via numerical simulation

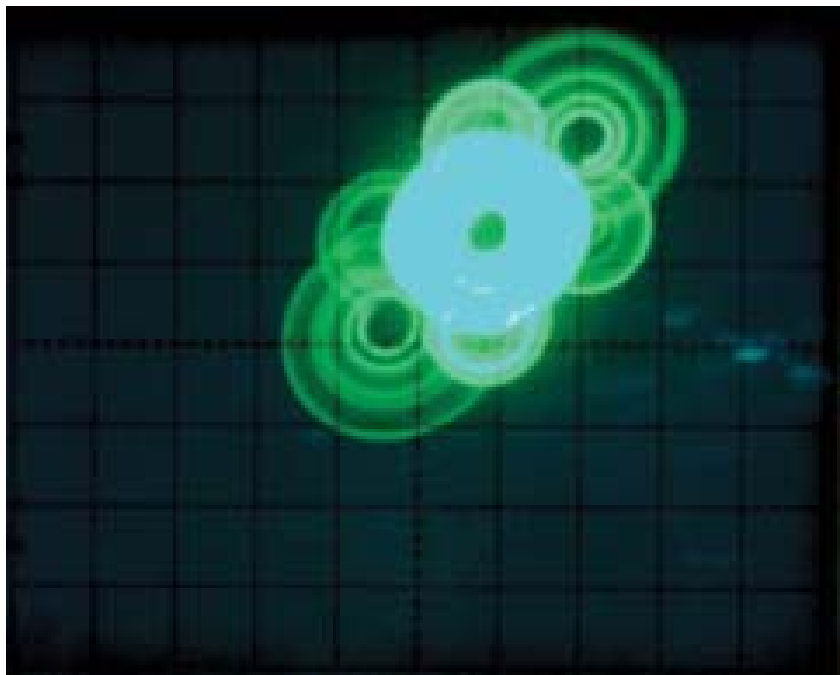

(b) Experimental observation of 7-scroll chaotic attractor [Li et al., 2003]

Fig. 54. 7-Scroll chaotic attractor.

where

$$
w(\zeta)= \begin{cases}0 & \zeta<\frac{V_{C C}}{m} \\ i \frac{V_{C C}}{m} & (2 i-1) \frac{V_{C C}}{m} \leq \zeta<(2 i+1) \frac{V_{C C}}{m} \quad \text { for } 1 \leq i \leq n-1 \\ n \frac{V_{C C}}{m} & \zeta \geq(2 n-1) \frac{V_{C C}}{m} .\end{cases}
$$

Let $\tau=t / \tau_{0}, 1 / \tau_{0}=1 / R C, R=3 \mathrm{k} \Omega, C=100 \mathrm{pF}$, $V_{B P}=1 \mathrm{~V}, x=v_{1} / V_{B p}, y=v_{2} / V_{B p}, z=v_{3} / V_{B p}$. Then, one obtains a system of dimensionless state equations as follows:

$$
\left\{\begin{array}{l}
\frac{d x}{d \tau}=y-w(y+z) \\
\frac{d y}{d \tau}=-x+a y+w(x+z)-a w(y+z) \\
\frac{d z}{d \tau}=-z+b w(x+z)+b w(y+z),
\end{array}\right.
$$

where

$$
w(\zeta)=\frac{1}{2} \sum_{i=0}^{n} u(\zeta-0.5-i),
$$

in which $u(\cdot)$ is the unit step function.

When $a=0.1, b=0.5$ and $w(\zeta)=0.5 u(\zeta-$ $0.5)+0.5 u(\zeta-1.5)$, system $(45)$ has a 7 -scroll chaotic attractor, as shown in Fig. 54(a). Let $V_{C C}=$ $5 \mathrm{~V}, R=R_{f 12}=R_{f 21}=R_{f 33}=3 \mathrm{k} \Omega, R_{f 22}=$ $30 \mathrm{k} \Omega, R_{i}=30 \mathrm{k} \Omega, C_{1}=C_{2}=C_{3}=C=$ $100 \mathrm{pF}, m=10, n=2$. The power supply of the operational amplifier is $\pm V_{C C}$ and the power supply of the comparator is $-V_{C C} \sim 0$. Figure 54(b) shows the experimental observation of a 7 -scroll chaotic attractor.

\section{Design of Multifolded Torus Chaotic Attractors}

In this section, a simple multifolded torus chaotic system with PWL nonlinearity is presented.

\subsection{A modified multifolded torus chaotic system}

Matsumoto et al. [1987] introduced a double-folded torus chaotic circuit, called folded torus circuit [Inaba, 1992; Matsumoto, 1987; Matsumoto et al., 1987], described by

$$
\left\{\begin{array}{l}
\dot{x}=-\alpha g(y-x) \\
\dot{y}=-g(y-x)-z \\
\dot{z}=\beta y,
\end{array}\right.
$$

where $g(y-x)=m_{1}(y-x)+1 / 2\left(m_{0}-m_{1}\right)(\mid y-x+$ $\left.x_{1}|-| y-x-x_{1} \mid\right)$ is a PWL odd function satisfying 
$g(x-y)=-g(y-x)$. When $\alpha=15, \beta=1$, $m_{0}=0.1, m_{1}=-0.07$, and $x_{1}=1$, system (46) has a double-folded torus chaotic attractor with the maximum Lyapunov exponent 0.0270.

To generate multifolded torus chaotic attractors, Yu et al. [2004a, 2005c, 2005e] proposed a modified multifolded torus circuit, described by

$$
\left\{\begin{array}{l}
\dot{x}=-\alpha g(y-x) \\
\dot{y}=-g(y-x)-z \\
\dot{z}=\beta y,
\end{array}\right.
$$

where $\alpha, \beta$ are real parameters, and $g(y-x)=$ $m_{N-1}(y-x)+1 / 2 \sum_{i=1}^{N-1}\left(m_{i-1}-m_{i}\right)\left(\left|y-x+x_{i}\right|-\right.$ $\left.\left|y-x-x_{i}\right|\right)$ is a PWL odd function satisfying $g(x-y)=-g(y-x)$.

Here, the PWL function $g(y-x)$ can be rewritten as

$$
\begin{aligned}
& g(y-x)= \\
& \left\{\begin{array}{l}
\text { if }|y-x|<x_{1}, \\
m_{0}(y-x) \\
\text { if } x_{i} \leq|y-x| \leq x_{i+1}, 1 \leq i \leq N-2, \\
m_{i}(y-x)+\sum_{j=1}^{i}\left(m_{j-1}-m_{j}\right) \operatorname{sgn}(y-x) x_{j} \\
\text { if }|y-x|>x_{n-1}, \\
m_{N-1}(y-x)+\sum_{j=1}^{N-1}\left(m_{j-1}-m_{j}\right) \operatorname{sgn}(y-x) x_{j},
\end{array}\right.
\end{aligned}
$$

where $m_{i}(0 \leq i \leq N-1)$ are the slopes of the segments or radials in various piecewise subregions,

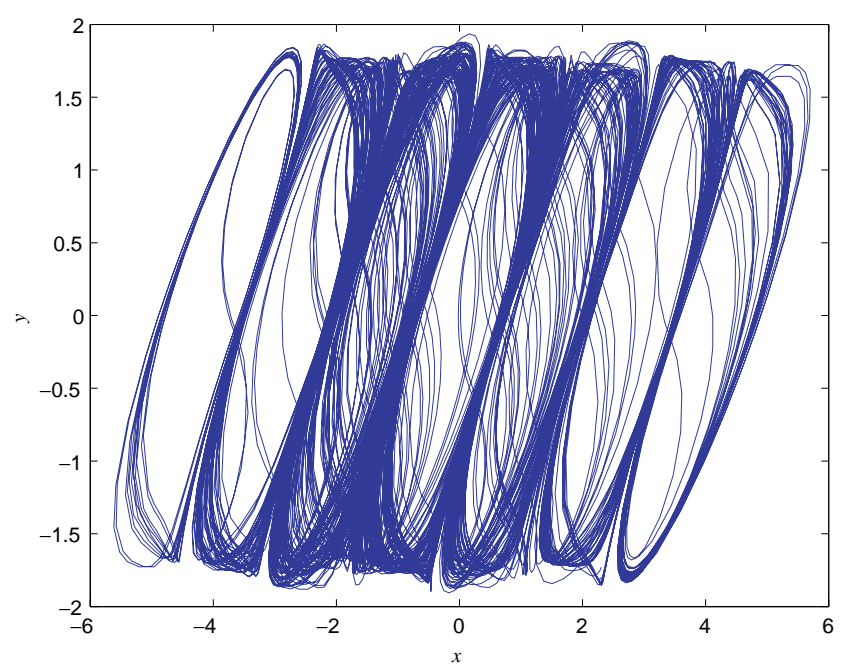

(a) 7-Folded torus attractor and $\pm x_{i}\left(x_{i} \geq 0,1 \leq i \leq N-1\right)$ are the switching points.

Assume that $\alpha=14.5, \beta=1.25$, and $x_{1}=0.75$. When $N=4, m_{0}=0.15, m_{1}=-0.17, m_{2}=0.15$, $m_{3}=-0.17, x_{2}=2.0735, x_{3}=3.5735$, system $(47)$ has a 7 -folded torus chaotic attractor, as shown in Fig. 55(a). The maximum Lyapunov exponent of this 7-folded torus chaotic attractor is 0.0901, calculated by the method available in [Lü et al., 2002e]. Similarly, when $N=5, m_{0}=-0.17, m_{1}=0.15$, $m_{2}=-0.17, m_{3}=0.15, m_{4}=-0.17, x_{2}=2.45$, $x_{3}=3.95, x_{4}=5.65$, system (47) has a 9-folded torus chaotic attractor, as shown in Fig. 55(b). The maximum Lyapunov exponent of this 9-folded torus chaotic attractor is 0.0730 , calculated by the method available in [Lü et al., 2002e].

Note that system (47) can create a maximum of $(2 N-1)$-folded torus chaotic attractor for $N>1$. In particular, every torus corresponds to a unique segment or radial of the PWL function $g(x)$. Moreover, theoretical analysis and numerical simulation both show that the slopes of the two radials of the PWL function $g(x)$ must be negative.

\subsection{Circuit implementation}

$\mathrm{Yu}$ et al. [2005c, 2005e] also designed a circuit diagram to experimentally verify the multifolded torus chaotic attractors obtained above. Figure 56 shows the circuitry, where the subcircuitry $N_{S}$ is the subtraction generator and its output is $\left(v_{C 2}-v_{C 1}\right)$, and the subcircuitry $N_{R}$ is the generator of the

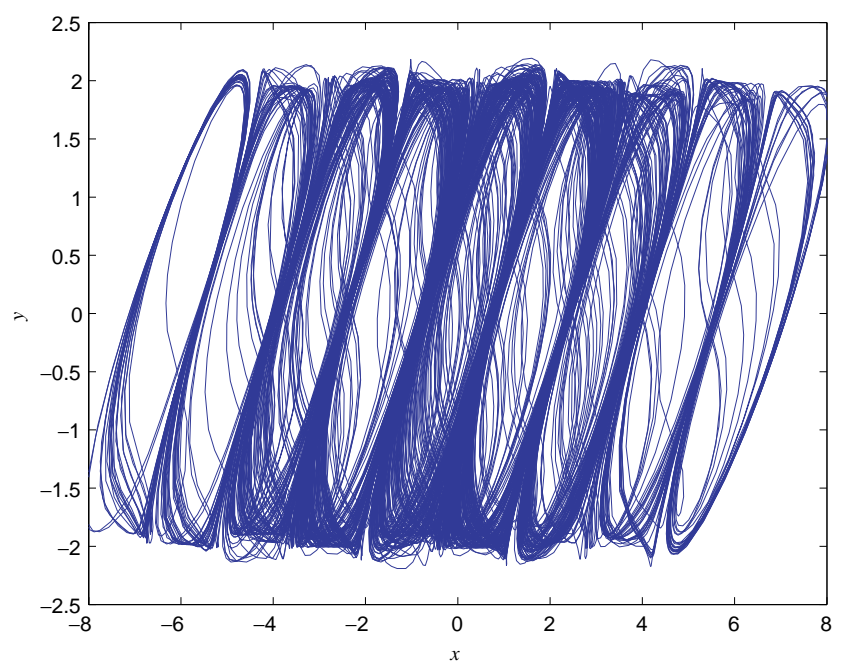

(b) 9-Folded torus attractor

Fig. 55. Numerical simulations for multifolded torus chaotic attractors. 


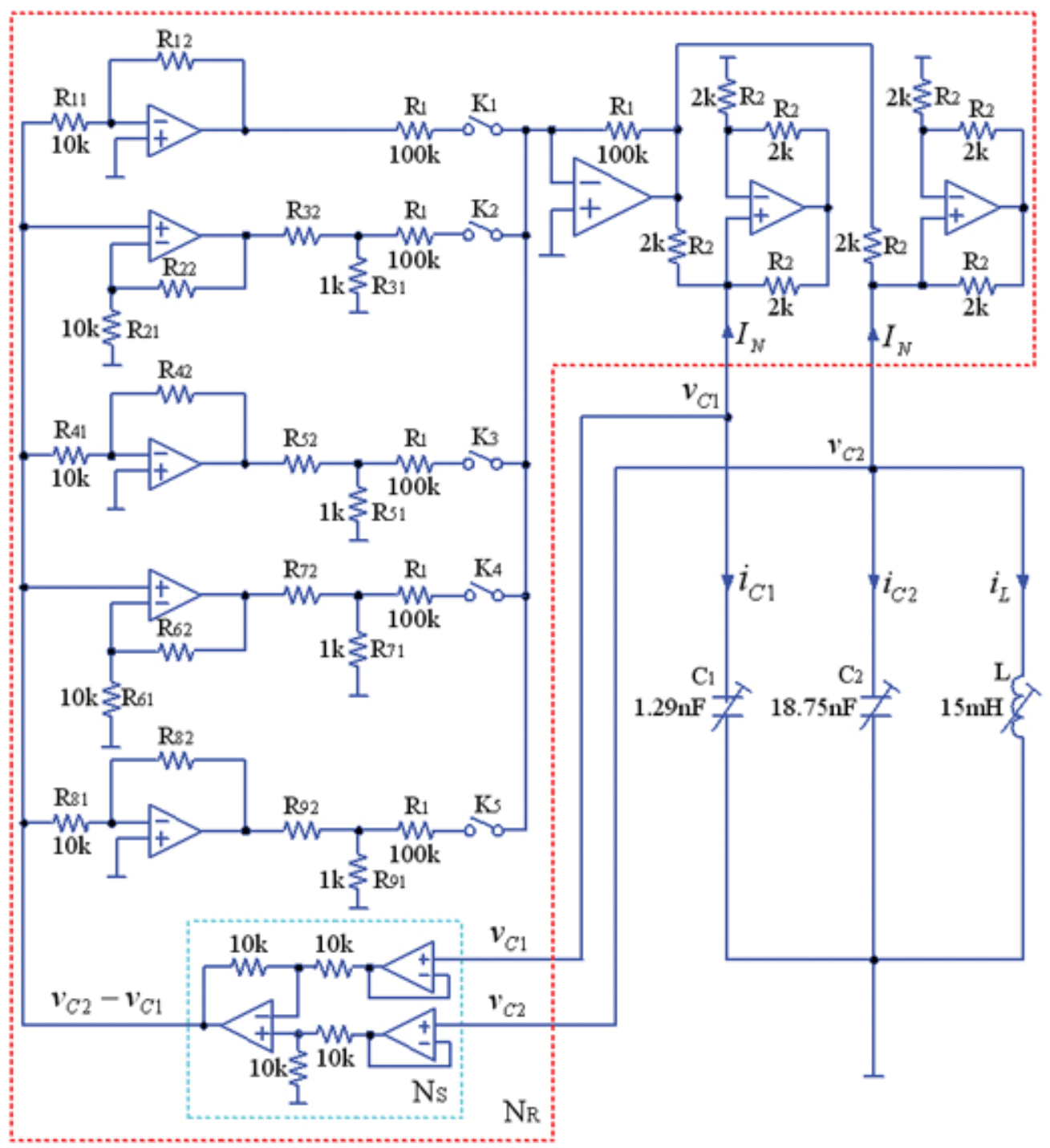

Fig. 56. Circuit diagram for multifolded torus chaotic attractors.

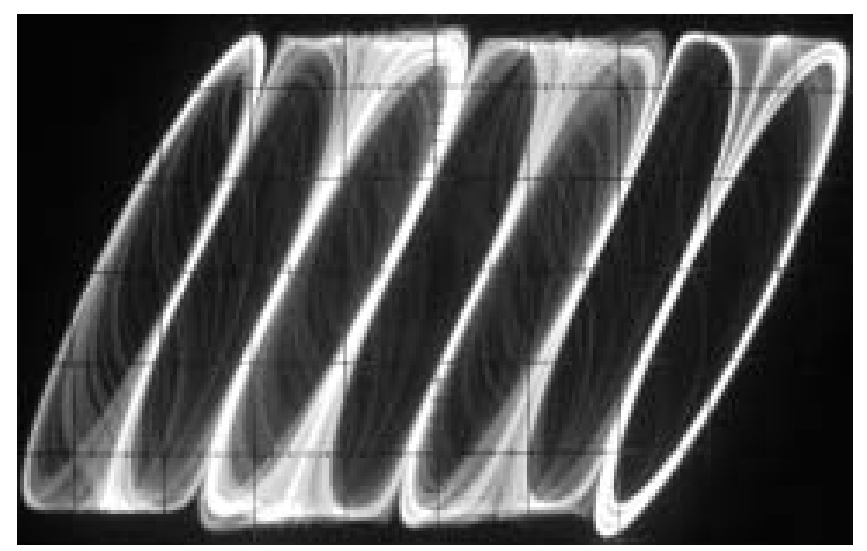

(a) 7-Folded torus, where $x=1.25 \mathrm{~V} / \mathrm{div}$ and $y=0.64 \mathrm{~V} / \operatorname{div}$

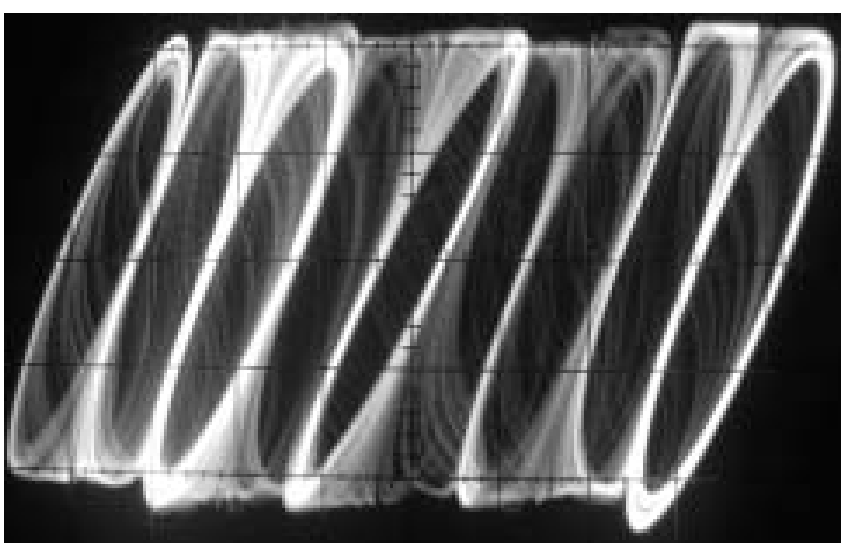

(b) 9-Folded torus, where $x=1.6 \mathrm{~V} /$ div and $y=0.9 \mathrm{~V} / \operatorname{div}$

Fig. 57. Experimental observations of multifolded torus chaotic attractors. 
PWL function $f\left(v_{C 2}-v_{C 1}\right)$ and its input and output satisfy the condition $I_{N}=f\left(v_{C 2}-v_{C 1}\right)$. Furthermore, one can rigorously calculate the theoretical values of all resistors in $N_{R}$ by using the recursive formulas available in [Yu et al., 2005c, 2005e]. The operational amplifier is selected to be TL082, and the supply voltage of electrical source is $\pm E_{C}= \pm 15 \mathrm{~V}$. Thus, the saturating voltage of the operational amplifier is $E_{\text {sat }}=14.3 \mathrm{~V}$. Figure 57 displays the experimental observations of 7, 9-folded torus chaotic attractors.

\section{Design of Hyperchaotic Multiscroll Attractors}

In this section, several typical approaches for generating hyperchaotic multiscroll attractors are discussed.

\subsection{Hyperchaotic multiscroll attractors from a simple four-dimensional system}

A simple 4D modified Chua's circuit can be used to generate complex $n$-scroll chaotic attractors [Yu et al., 2003a, 2004b].

Yin [1996] introduced a modified Chua's circuit, as shown in Fig. 58(a), which is different from the original Chua's circuit in that a $R C$ parallel circuit consisting of $R_{3}$ and $C_{3}$ is added into the $L$-arm of the original circuit. This modified Chua's circuit is described by

$$
\left\{\begin{array}{l}
\dot{x}=\alpha(y-x-g(x)) \\
\dot{y}=x-y+z \\
\dot{z}=-\beta(y-w) \\
\dot{w}=-\gamma_{2}\left(z+\gamma_{1} w\right),
\end{array}\right.
$$

where

$$
\begin{aligned}
g(x)= & m_{1} x+\frac{1}{2}\left(m_{0}-m_{1}\right) \\
& \times\left(\left|x+b_{1}\right|-\left|x-b_{1}\right|\right) .
\end{aligned}
$$

When $\alpha=9.934, \beta=14.334, \gamma_{1}=27.333, \gamma_{2}=$ $0.0497, m_{0}=-1.246, m_{1}=-0.6724$, and $b_{1}=10$, system (48) has a double-scroll chaotic attractor, as shown in Fig. 58(b).

$\mathrm{Yu}$ et al. [2003a, 2004b] further extended the PWL function (49) of the 4D modified Chua's circuit, for generating $n$-scroll chaotic attractors, as

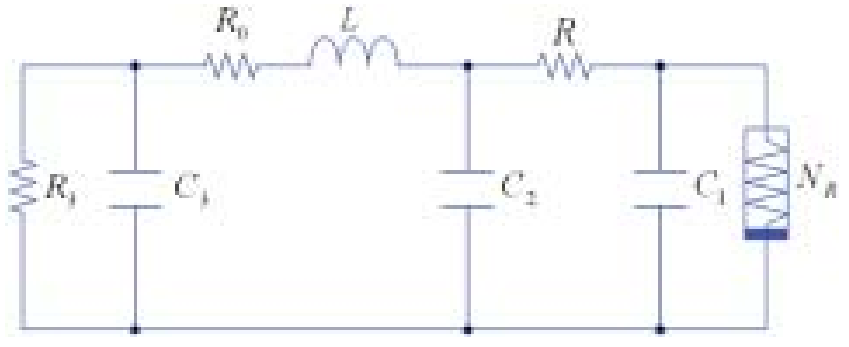

(a) The modified Chua's circuit

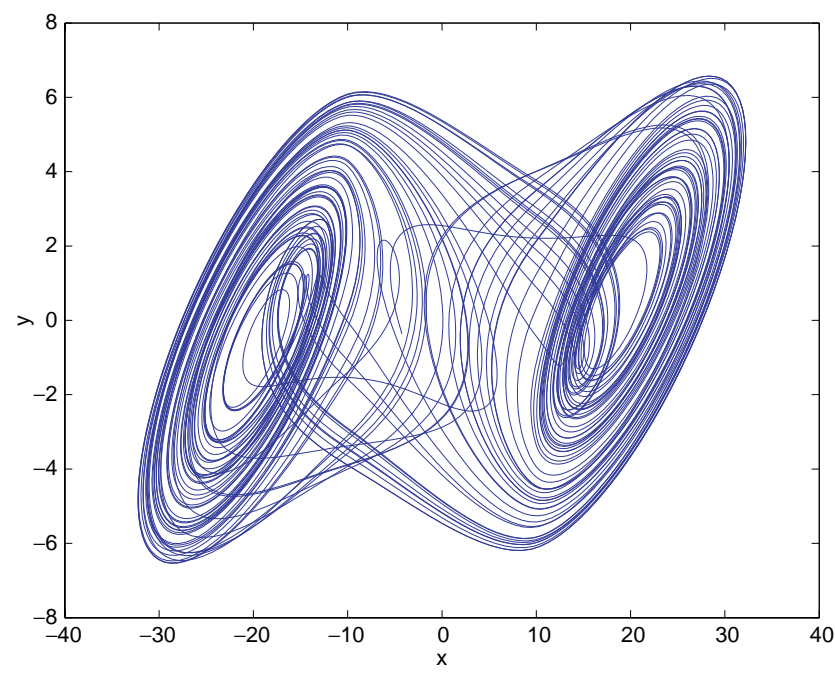

(b) Double-scroll chaotic attractor

Fig. 58. The 4D modified Chua's circuit.

follows:

$$
\begin{aligned}
g(x)= & m_{N-1} x+\frac{1}{2} \sum_{i=1}^{N-1} \\
& \times\left(m_{i-1}-m_{i}\right)\left(\left|x+b_{i}\right|-\left|x-b_{i}\right|\right) .
\end{aligned}
$$

When $\alpha=9.934, \beta=14.334, \gamma_{1}=27.333$, $\gamma_{2}=0.0497, m_{0}=m_{2}=m_{4}=-1.246, m_{1}=$ $m_{3}=m_{5}=-0.6724$, and $b_{1}=10, b_{2}=29.2466$, $b_{3}=49.2466, b_{4}=68.4931, b_{5}=88.4931$, system (48) with (50) has a 6-scroll chaotic attractor, as shown in Fig. 59(a); when $\alpha=9.934, \beta=14.334$, $\gamma_{1}=27.333, \gamma_{2}=0.0497, m_{0}=m_{2}=m_{4}=m_{6}=$ $m_{8}=m_{10}=-1.246, m_{1}=m_{3}=m_{5}=m_{7}$ $=m_{9}=m_{11}=-0.6724$, and $b_{1}=12.4, b_{2}=$ $36.2657, b_{3}=61.0657, b_{4}=84.9315, b_{5}=109.7315$, $b_{6}=133.5972, b_{7}=158.3972, b_{8}=182.2629$, $b_{9}=207.0629, b_{10}=230.9287, b_{11}=279.5944$, system (48) with (50) has a 12-scroll chaotic attractor, as shown in Fig. 59(b). 


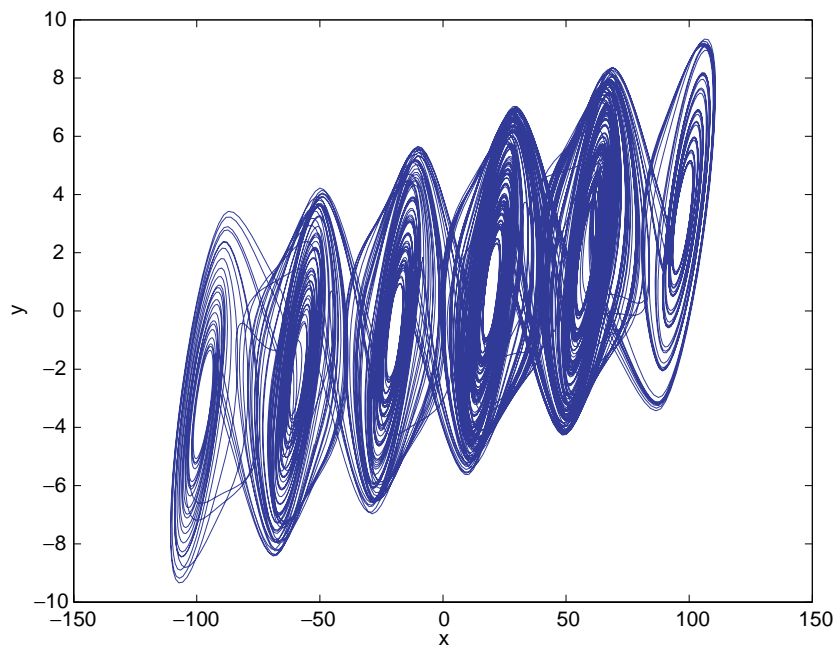

(a) 6-Scroll chaotic attractor

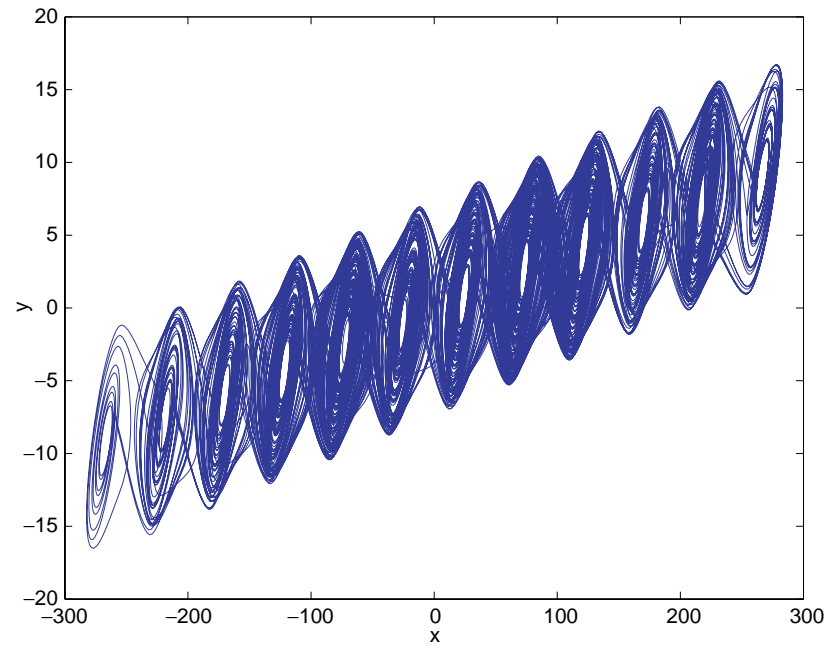

(b) 12-Scroll chaotic attractor

Fig. 59. $n$-Scroll chaotic attractors from the 4D modified Chua's circuit.

To experimentally verify the $n$-scroll chaotic attractors simulated above, Yu et al. [2004b] recast the modified Chua's circuit (48) with (50) as follows:

$$
\left\{\begin{array}{l}
\left.\frac{d V_{C 1}}{d t}=\frac{1}{R C_{1}}\left(V_{C 2}-V_{C 1}\right)-\frac{1}{C_{1}} f\left(V_{C 1}\right)\right) \\
\frac{d V_{C 2}}{d t}=\frac{1}{R C_{2}}\left(V_{C 1}-V_{C 2}\right)+\frac{1}{C_{2}} i_{L} \\
\frac{d i_{L}}{d t}=-\frac{1}{L}\left(V_{C 3}-V_{C 2}\right) \\
\frac{d V_{C 3}}{d t}=-\frac{1}{C_{3}} i_{L}-\frac{1}{R_{0} C_{3}} V_{C 3},
\end{array}\right.
$$

where

$$
\begin{aligned}
f\left(V_{C 1}\right)= & \frac{m_{N-1}}{R} V_{C 1}+\frac{1}{2} \sum_{i=1}^{N-1}\left(\frac{m_{i-1}}{R}-\frac{m_{i}}{R}\right) \\
& \times\left(\left|V_{C 1}+E_{i}\right|-\left|V_{C 1}-E_{i}\right|\right) \\
= & G_{N-1} V_{C 1}+\frac{1}{2} \sum_{i=1}^{N-1}\left(G_{i-1}-G_{i}\right) \\
& \times\left(\left|V_{C 1}+E_{i}\right|-\left|V_{C 1}-E_{i}\right|\right)
\end{aligned}
$$

and $C_{2} / C_{1}=\alpha, R^{2} C_{2} / L=\beta, R / R_{0}=\gamma_{1}, C_{2} / C_{3}=$ $\gamma_{2}, V_{C 1} / B_{P}=x, V_{C 2} / B_{P}=y, i_{L} R / B_{P}=z$, $V_{C 3} / B_{P}=w, t / R C_{2}=\tau, B_{P}=1 \mathrm{~V}, f\left(V_{C 1}\right)=$ $g\left(V_{C 1}\right) / R, E_{i}=x_{i} B_{P}, G_{i}=m_{i} / R$.

$\mathrm{Yu}$ et al. [2004b] then constructed a circuit to physically realize 4,6 -scroll chaotic attractors.
Figure 60 [Yu et al., 2004b] shows the circuit diagram, where all operational amplifiers are TL082, the function block within the dotted line is corresponding to a diode $N_{R}$ in Chua's circuit, and each operational amplifier and its associated resistors contribute a subcurrent of $N_{R}$ with switching voltage $E_{i}(i=1,2,3,4,5)$. When $K$ is switched on, the circuit generates a 6 -scroll chaotic attractor; when $K$ is switched off, the circuit creates a 4-scroll chaotic attractor. The voltages of electronic sources are $\pm 15 \mathrm{~V}$, and their corresponding saturating voltages are $\pm 13.5 \mathrm{~V}$. All circuit parameters are shown in Fig. 60. Figures 61(a) and 61(b) [Yu et al., 2004b] display the observed 4- and 6-scroll chaotic attractors.

\subsection{Hyperchaotic multiscroll attractors from the modified MCK circuit}

Matsumoto et al. [1986] introduced the first hyperchaotic circuit, called MCK circuit, described by

$$
\left\{\begin{array}{l}
C_{1} \frac{d v_{C_{1}}}{d t}=g\left(v_{C_{2}}-v_{C_{1}}\right)-i_{L_{1}} \\
C_{2} \frac{d v_{C_{2}}}{d t}=-g\left(v_{C_{2}}-v_{C_{1}}\right)-i_{L_{2}} \\
L_{1} \frac{d i_{L_{1}}}{d t}=v_{C_{1}}+R i_{L_{1}} \\
L_{2} \frac{d i_{L_{2}}}{d t}=v_{C_{2}}
\end{array}\right.
$$




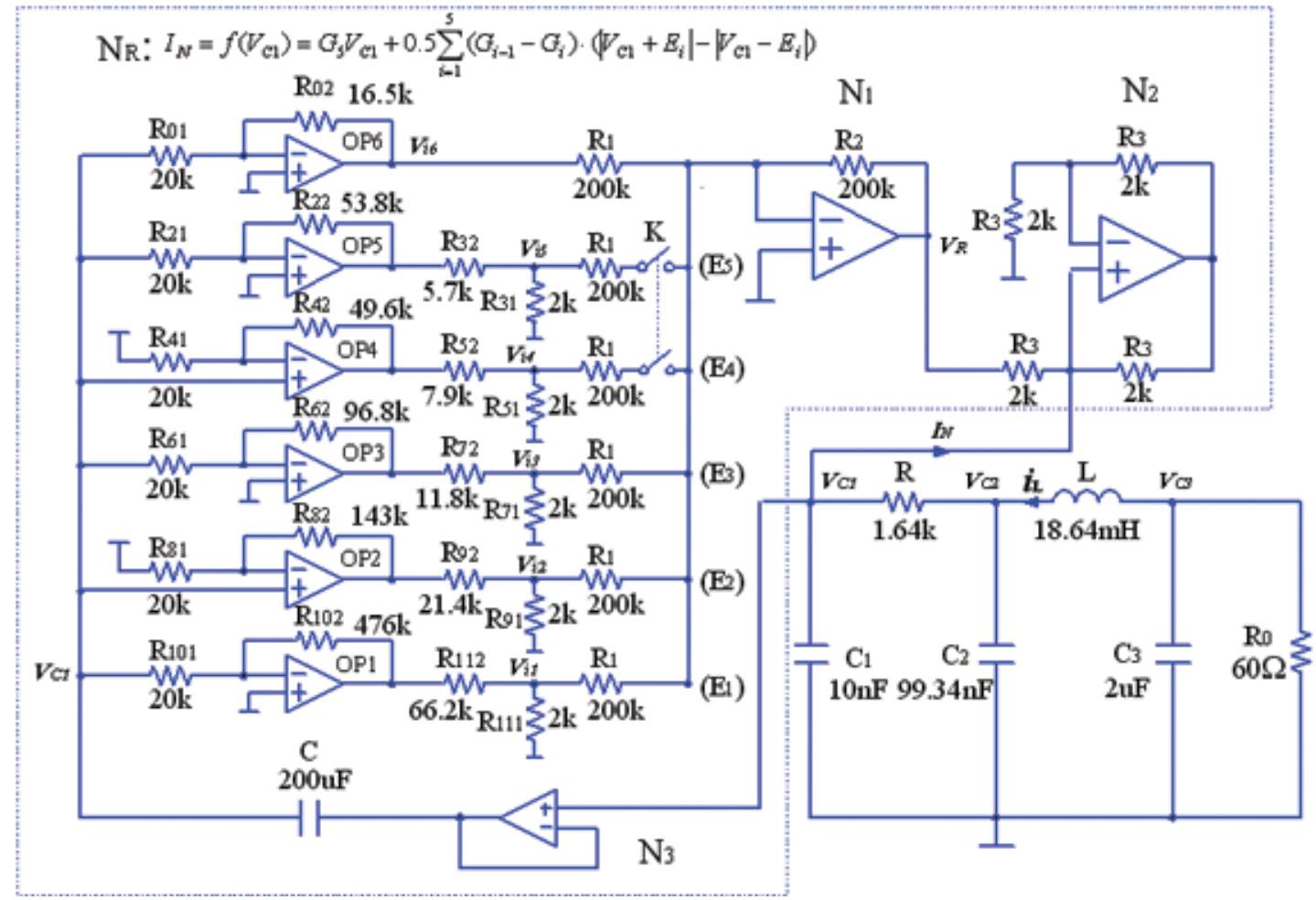

Fig. 60. Circuit diagram of the 4D modified Chua's circuit [Yu et al., 2004b].

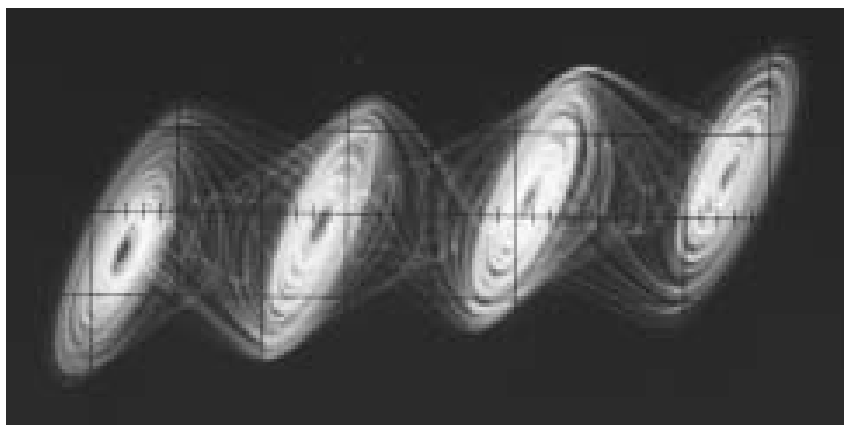

(a) 4-Scroll chaotic attractor

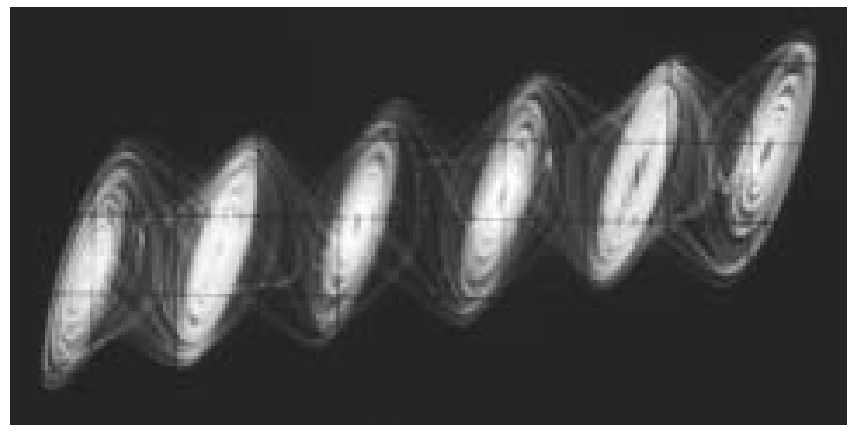

(b) 6-Scroll chaotic attractor

Fig. 61. Experimental observations of 4, 6-scroll chaotic attractors [Yu et al., 2004b].

where

$$
\begin{gathered}
g\left(v_{C_{2}}-v_{C_{1}}\right)=m_{0}\left(v_{C_{2}}-v_{C_{1}}\right)+0.5\left(m_{1}-m_{0}\right) \\
\times\left(\left|v_{C_{2}}-v_{C_{1}}-1\right|-\left|v_{C_{2}}-v_{C_{1}}+1\right|\right) .
\end{gathered}
$$

Figure 62(a) [Matsumoto et al., 1986] shows the MCK hyperchaotic circuit, where the nonlinear resistor is characterized as shown in Fig. 62(b) [Matsumoto et al., 1986], and all other electronic devices are linear and passive except a negative resistance $-R$. Figure 63(a) [Matsumoto et al., 1986] realizes the MCK hyperchaotic circuit, where $N_{1}$ realizes the nonlinear resistor and $N_{2}$ realizes the negative resistance. When $C_{1}=0.5, C_{2}=0.05$,
$L_{1}=1, L_{2}=2 / 3, R=1, m_{0}=3, m_{1}=-0.2$, the observed hyperchaotic attractor is shown in Fig. 63(b) [Matsumoto et al., 1986]. The Lyapunov exponents are $\lambda_{1}=0.24, \lambda_{2}=0.06, \lambda_{3}=0, \lambda_{4}=$ -53.8 and the Lyapunov dimension is $d_{L}=3.006$.

The dimensionless state equation of the MCK circuit (53) is given by

$$
\left\{\begin{array}{l}
\dot{x}=\alpha(g(y-x)-z) \\
\dot{y}=\beta(-g(y-x)-w) \\
\dot{z}=x+z \\
\dot{w}=\gamma y
\end{array}\right.
$$




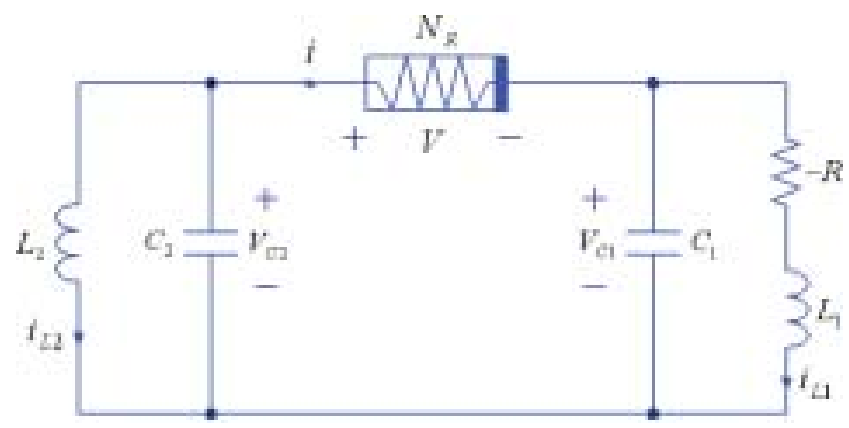

(a) MCK circuit

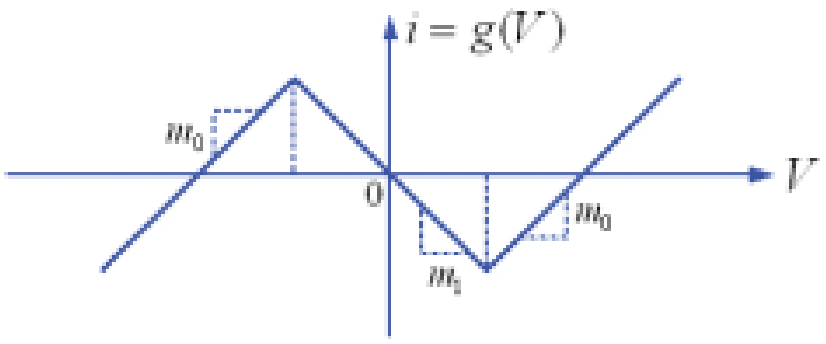

(b) $v-i$ characteristic of $N_{1}$

Fig. 62. 4D MCK hyperchaotic circuit [Matsumoto et al., 1986].

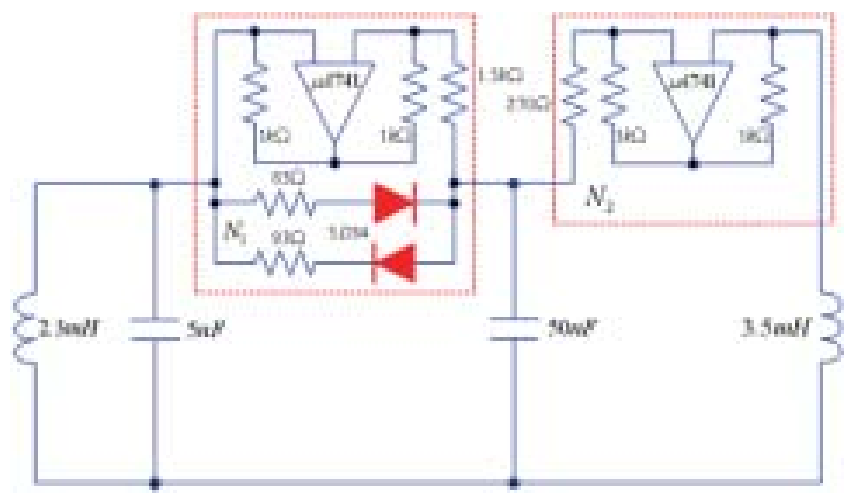

(a) Circuit diagram

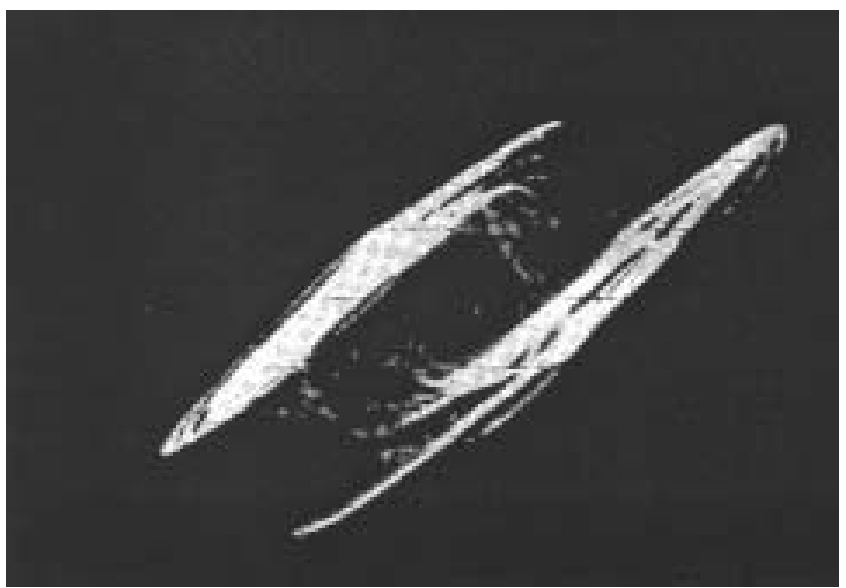

(b) Experimental observation

Fig. 63. Realization of MCK hyperchaotic circuit [Matsumoto et al., 1986].

where

$$
\begin{aligned}
g(y-x)= & m_{0}(y-x)+\frac{1}{2}\left(m_{1}-m_{0}\right) \\
& \times(|y-x+1|-|y-x-1|) .
\end{aligned}
$$

To generate $n$-double scroll hyperchaotic attractors from (55), Yalcin et al. [2000a] modified the PWL characteristic (56) as follows:

$$
\begin{aligned}
g(y-x)= & m_{0}(y-x)+\frac{1}{2} \sum_{i=1}^{2 n-1}\left(m_{i}-m_{i-1}\right) \\
& \times\left(\left|y-x+c_{i}\right|-\left|y-x-c_{i}\right|\right)
\end{aligned}
$$

Moreover, $\mathrm{Yu}$ et al. [2004b, 2005b] further extended (57) to create $n$-scroll hyperchaotic attractors, as follows:

$$
\begin{aligned}
g(y-x)= & m_{N-1}(y-x)+\frac{1}{2} \sum_{i=1}^{N-1}\left(m_{i-1}-m_{i}\right) \\
& \times\left(\left|y-x+c_{i}\right|-\left|y-x-c_{i}\right|\right)
\end{aligned}
$$

When $\alpha=2, \beta=20, \gamma=1.5, m_{0}=m_{2}=$ $m_{4}=m_{6}=m_{8}=3, m_{1}=m_{3}=m_{5}=-0.9$, $m_{7}=-0.8, c_{1}=1, c_{2}=3.2105, c_{3}=5.5205$, $c_{4}=8.1953, c_{5}=10.9904, c_{6}=14.2268, c_{7}=$ $17.7699, c_{8}=22.9435$, system (55) with (58) can generate a 9-scroll hyperchaotic attractor, as shown in Fig. 64(a); when $\alpha=2, \beta=20, \gamma=1.5$, $m_{0}=m_{2}=m_{4}=m_{6}=-0.9, m_{8}=-0.8$, $m_{1}=m_{3}=m_{5}=m_{7}=m_{9}=2.9, c_{1}=1, c_{2}=3.1$, $c_{3}=5.41, c_{4}=7.951, c_{5}=10.7461, c_{6}=13.8207$, $c_{7}=17.2028, c_{8}=21.2774, c_{9}=16.9686$, system (55) with (58) can generate a 10-scroll hyperchoatic attractor, as shown in Fig. 64(b).

Recently, Yu et al. [2005b] designed a novel circuit diagram to experimentally verify 2,3,4-scroll hyperchaotic attractors. Figure 65 displays the circuitry, where $N 1$ is the generator of the negative resistor $-R$, and $N_{R}$ is the multiple PWL function generator satisfying $I_{N}=f\left(v_{C 2}-v_{C 1}\right)$. All operational amplifiers are TL082. The voltage of 


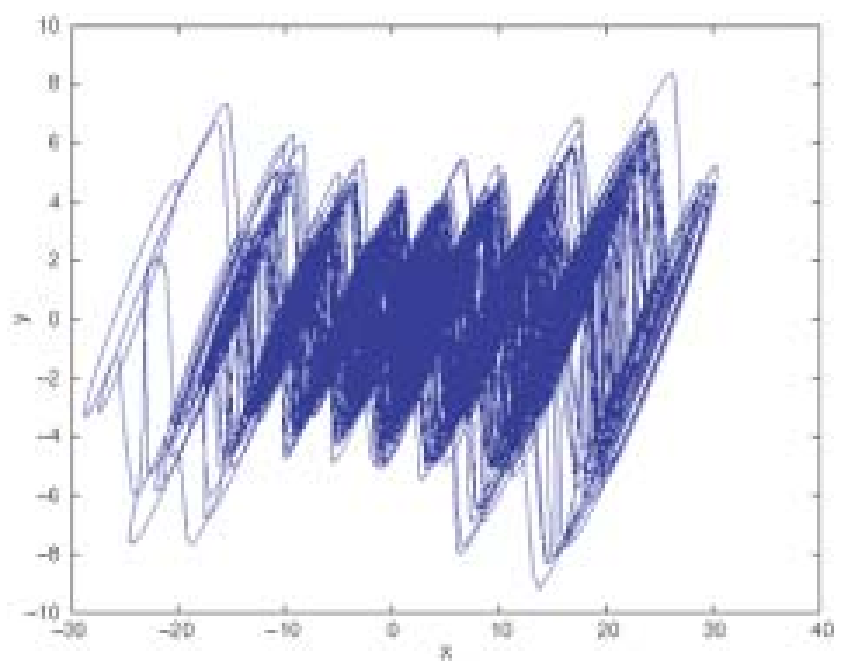

(a) 9-Scroll hyperchaotic attractor

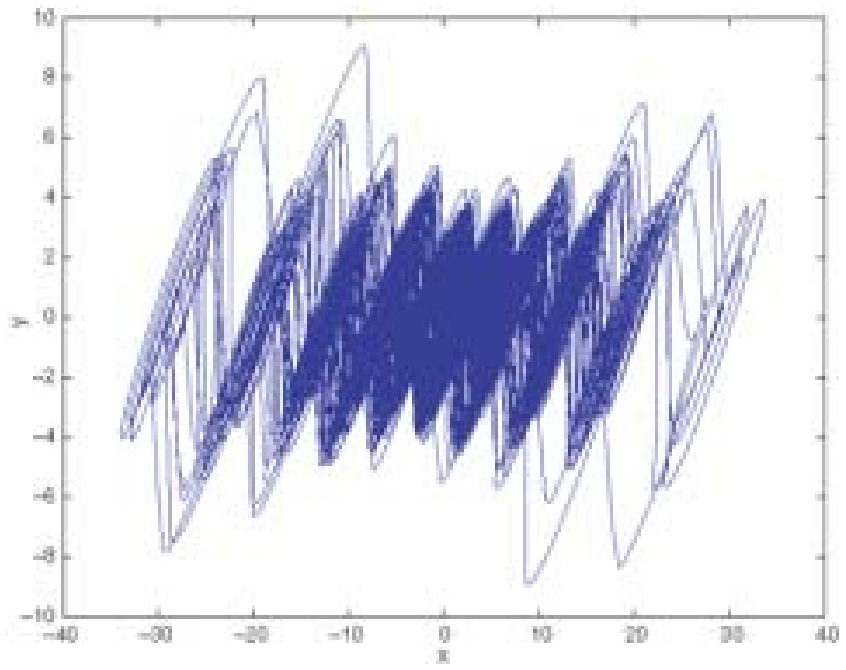

(b) 10-Scroll hyperchaotic attractor

Fig. 64. $n$-Scroll hyperchaotic attractors from the modified MCK circuit.

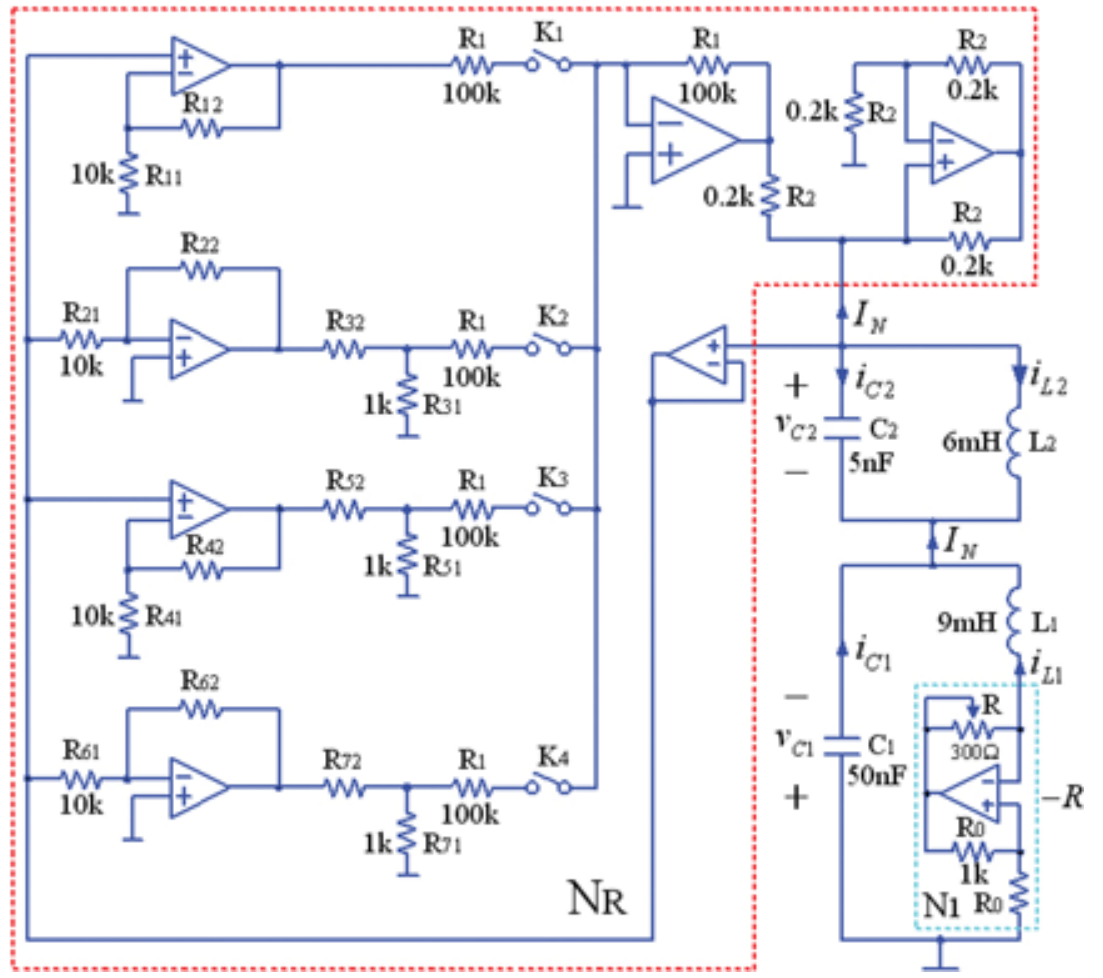

Fig. 65. Circuit diagram for the realization of 2,3,4-scroll hyperchaotic attractors.

the power supply is $E=15 \mathrm{~V}$ and the saturating voltages of the operation amplifiers are $E_{\text {sat }}=$ $14.3 \mathrm{~V}$. Let $R_{1}=100 \mathrm{k} \Omega, R_{2}=0.2 \mathrm{k} \Omega, R_{31}=R_{51}=$ $R_{71}=1 \mathrm{k} \Omega, R_{11}=R_{21}=R_{41}=R_{61}=10 \mathrm{k} \Omega$. According to Fig. 65 , when $K 1, K 2$ are switched on, $K 3, K 4$ are switched off, and $R_{12}=10 \mathrm{k}$, $R_{22}=286 \mathrm{k}, R_{32}=12.4 \mathrm{k}$, the circuit can generate a double-scroll hyperchaotic attractor; when $K 1$, $K 2, K 3$ are switched on, $K 4$ is switched off, and $R_{12}=10 \mathrm{k}, R_{22}=78 \mathrm{k}, R_{32}=2.08 \mathrm{k}, R_{42}=276 \mathrm{k}$, $R_{52}=10.29 \mathrm{k}$, the circuit can create a 3 -scroll hyperchaotic attractor, as shown in Fig. 66(a); when $K 1, K 2, K 3, K 4$ are switched on and $R_{12}=9.3 \mathrm{k}$, $R_{22}=47.3 \mathrm{k}, R_{32}=0.97 \mathrm{k}, R_{42}=83.5 \mathrm{k}, R_{52}=$ 


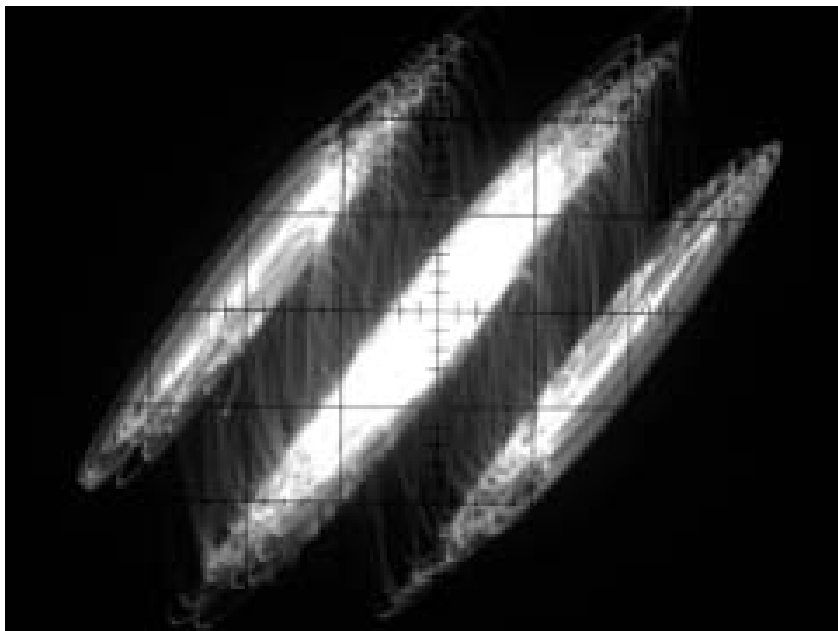

(a) 3-Scroll hyperchaotic attractor

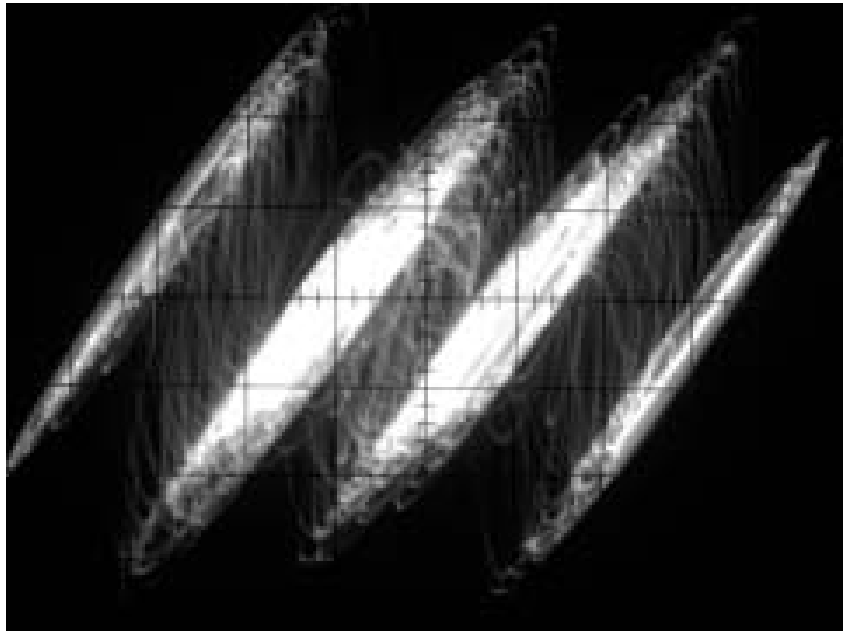

(b) 4-Scroll hyperchaotic attractor

Fig. 66. Experimental observations of 3, 4-scroll hyperchaotic attractors.

$2.9 \mathrm{k}, R_{62}=286 \mathrm{k}, R_{72}=10.9 \mathrm{k}$, the circuit can generate a 4-scroll hyperchaotic attractor, as shown in Fig. 66(b).

\subsection{Hyperchaotic multiscroll attractors from CNNs}

Suykens and Chua [1997] introduced a 1D-CNN for generating $n$-scroll hypercube attractors.

Assume that the CNN consists of $m$ identical generalized Chua's circuits (8), with unidirectional coupling between the second equations of cells, which is described by

$$
\left\{\begin{array}{l}
\dot{x}_{i}=\alpha\left(y_{i}-h\left(x_{i}\right)\right) \\
\dot{y}_{i}=x_{i}-y_{i}+z_{i}+k_{i-1}\left(y_{i}-y_{i-1}\right) \\
\dot{z}_{i}=-\beta y_{i}, \quad i=1,2, \ldots, m,
\end{array}\right.
$$

or with diffusive coupling between the first equations of cells as

$$
\left\{\begin{array}{l}
\dot{x}_{i}=\alpha\left(y_{i}-h\left(x_{i}\right)\right)+d_{x}\left(x_{i-1}-2 x_{i}+x_{i+1}\right) \\
\dot{y}_{i}=x_{i}-y_{i}+z_{i} \\
\dot{z}_{i}=-\beta y_{i}, \quad i=1,2, \ldots, m
\end{array}\right.
$$

or with diffusive coupling between second first equations of cells as

$$
\left\{\begin{array}{l}
\dot{x}_{i}=\alpha\left(y_{i}-h\left(x_{i}\right)\right) \\
\dot{y}_{i}=x_{i}-y_{i}+z_{i}+d_{y}\left(x_{i-1}-2 x_{i}+x_{i+1}\right) \\
\dot{z}_{i}=-\beta y_{i}, \quad i=1,2, \ldots, m .
\end{array}\right.
$$

Moreover, assume that $y_{0}=y_{m}, k_{0}=0, k_{i}=k(i=$ $1, \ldots, m-1)$ for $(59)$ and $x_{0}=x_{m}, x_{m+1}=x_{1}$ for (60) and (61).

Kapitaniank and Chua [Yalcin, 2004] also investigated the unidirectional coupling CNN with $n=1$ for creating a double-double scroll attractor. In a similar way, by using $n$-scroll attractors as cells, Suykens and Chua [1997] obtained $n$-scroll hypercube attractors $(m \geq 4)$ with weak unidirectional and diffusive coupling in the common state subspace of the cells; that is, $\left(x_{1}, x_{2}\right)$ for $m=2$, $\left(x_{1}, x_{2}, x_{3}\right)$ for $m=3$, and so on. The dimension of the hypercube is equal to the number of cells $m$ and hyperchaos is obtained for $m>2$. The coupling coefficients $k, d_{x}, d_{y}$ are set as small as 0.01 and the initial values are chosen close to the origin. Figure 67 shows the 2-double scroll square and cube from a 1D-CNN of 2 and 3 cells, respectively, of 2-double scroll circuits with unidirectional coupling $k=0.002$ between the cells.

\subsection{Hyperchaotic coupled Chua's circuits}

Cafagna and Grassi [2003a, 2003b] introduced an approach for generating 2-D and 3-D hyperchaotic attractors from coupled Chua's circuits with the sine nonlinearity.

Two Chua's circuits (23) [Cafagna \& Grassi, 2003a] with sine nonlinearities are coupled in the following form: 


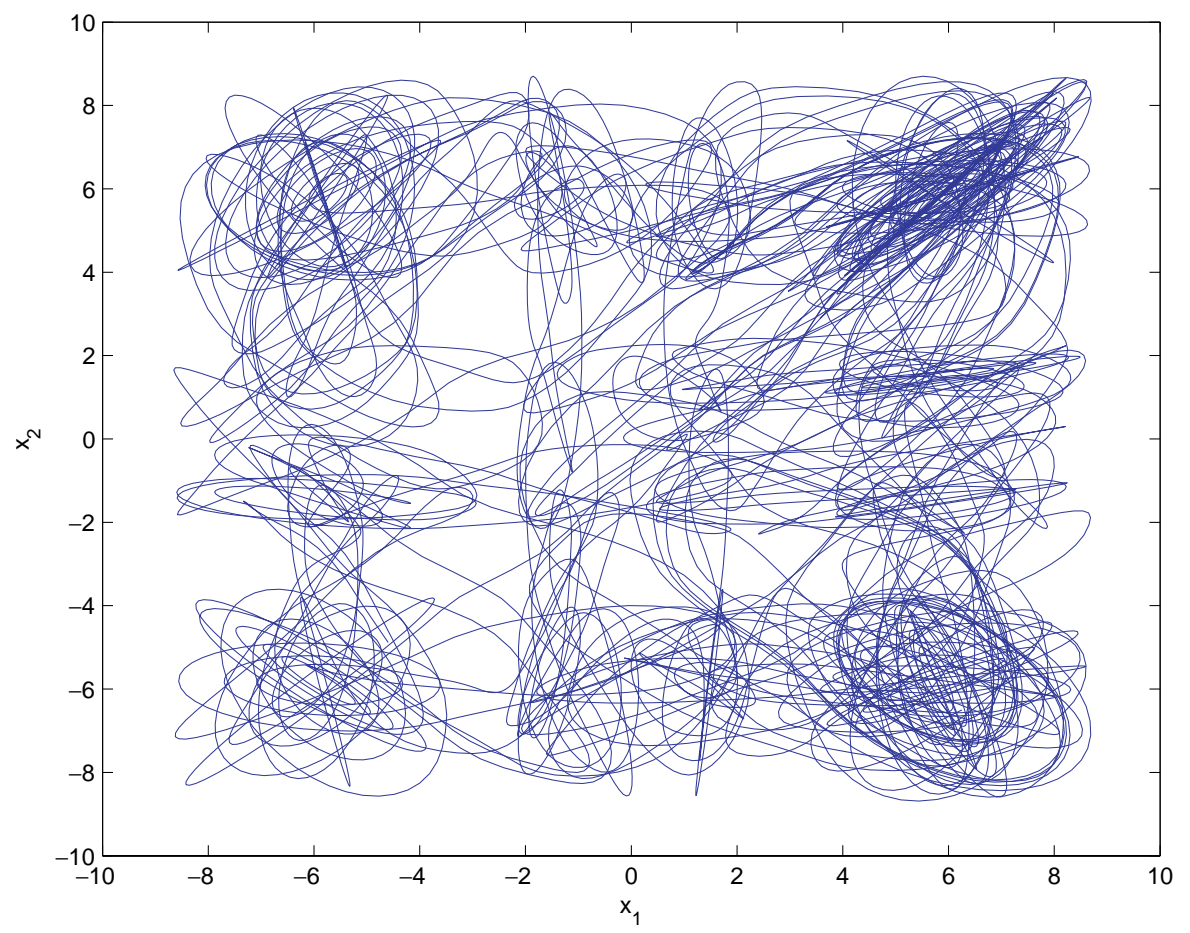

(a) 2-Double scroll square

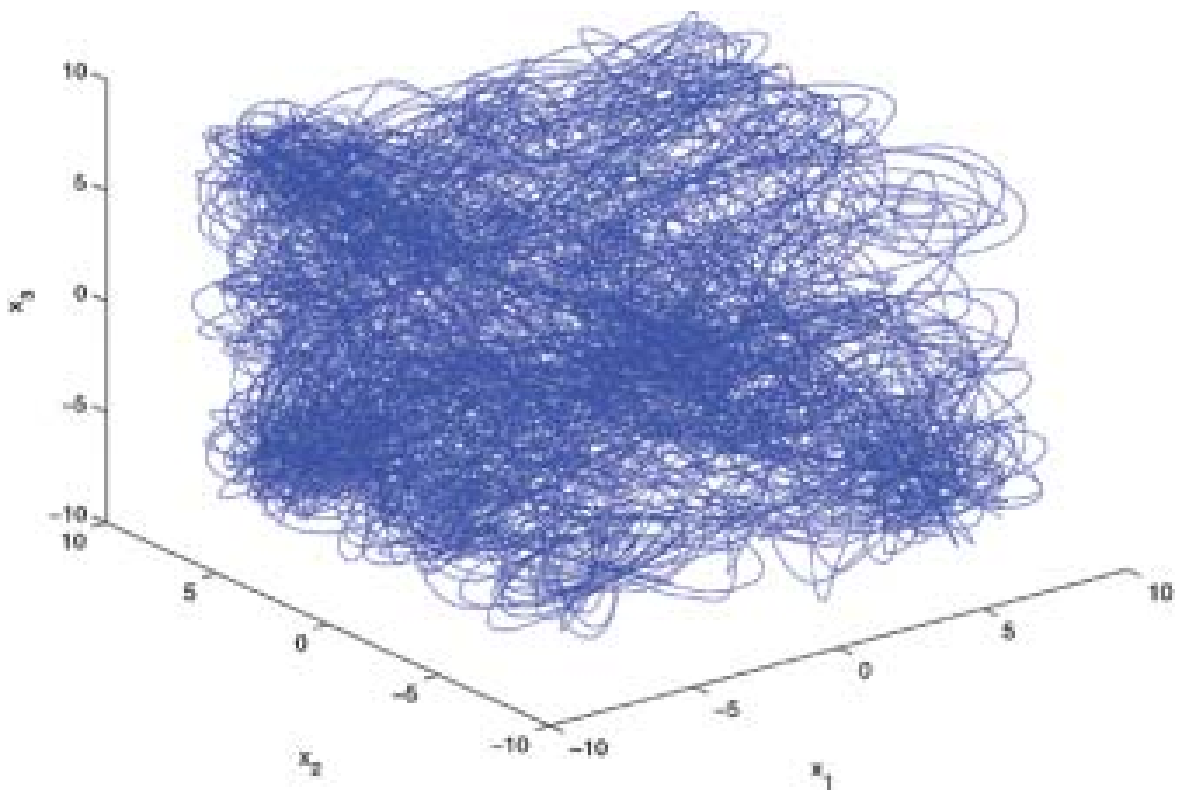

(b) 2-Double scroll cube

Fig. 67. 1D-CNN of 2, 3 cells of 2 -double scroll circuits with unidirectional coupling between the cells $(k=0.002)$. 


$$
\left\{\begin{array}{l}
\dot{x}_{1}=\alpha\left(x_{2}-f_{1}\left(x_{1}\right)\right) \\
\dot{x}_{2}=x_{1}-x_{2}+x_{3}+h\left(x_{5}-x_{2}\right) \\
\dot{x}_{3}=-\beta x_{2} \\
\dot{x}_{4}=\alpha\left(x_{5}-f_{2}\left(x_{4}\right)\right) \\
\dot{x}_{5}=x_{4}-x_{5}+x_{6}+h\left(x_{2}-x_{5}\right) \\
\dot{x}_{6}=-\beta x_{5}
\end{array}\right.
$$

where

$$
f_{1}\left(x_{1}\right)= \begin{cases}\frac{b \pi}{2 a}\left(x_{1}-2 a c_{1}\right) & x_{1} \geq 2 a c_{1} \\ -b \sin \left(\frac{\pi x_{1}}{2 a}+d_{1}\right) & -2 a c_{1}<x_{1}<2 a c_{1} \\ \frac{b \pi}{2 a}\left(x_{1}+2 a c_{1}\right) & x_{1} \leq-2 a c_{1}\end{cases}
$$

and

$$
f_{2}\left(x_{4}\right)= \begin{cases}\frac{b \pi}{2 a}\left(x_{4}-2 a c_{2}\right) & x_{4} \geq 2 a c_{2} \\ -b \sin \left(\frac{\pi x_{4}}{2 a}+d_{2}\right) & -2 a c_{2}<x_{4}<2 a c_{2} \\ \frac{b \pi}{2 a}\left(x_{4}+2 a c_{2}\right) & x_{4} \leq-2 a c_{2} .\end{cases}
$$

Here, $\alpha, \beta, a, b, c_{1}, c_{2}, d_{1}, d_{2}$ are real constants and $d_{i}(i=1,2)$ are given by

$$
d_{i}= \begin{cases}\pi & \text { for } c_{i} \text { is even } \\ 0 & \text { for } c_{i} \text { is odd }\end{cases}
$$

Figure 68 shows the coupling structure of two Chua's circuits with sine nonlinearities. Assume that $\alpha=10.814, \beta=14.0, a=1.3, b=0.11$, $h=0.25$ and $c_{1}, c_{2}, d_{1}, d_{2}$ are design parameters. System (62) can generate 2 -D $m \times n$-grid scroll hyperchaotic attractors for suitable design parameters. When $c_{1}=c_{2}=5, d_{1}=d_{2}=0$, system (62) has a 2-D $6 \times 6$-grid scroll hyperchaotic attractor, as shown in Fig. 69(a). When $c_{1}=c_{2}=8, d_{1}=$ $d_{2}=\pi$, system $(62)$ has a 2 -D $9 \times 9$-grid scroll hyperchaotic attractor, as shown in Fig. 69(b). For the $9 \times 9$-grid scroll attractor, the Lyapunov exponent spectrum is $L E_{1}=0.334, L E_{2}=$ $0.172, L E_{3}=0, L E_{4}=-0.169, L E_{5}=-1.347$, $L E_{6}=-1.749$.

Similarly, three Chua's circuits (23) [Cafagna \& Grassi, 2003b] with sine nonlinearities can be coupled in the following form:

$$
\left\{\begin{array}{l}
\dot{x}_{1}=\alpha\left(x_{2}-f_{1}\left(x_{1}\right)\right) \\
\dot{x}_{2}=x_{1}-x_{2}+x_{3}+h\left(x_{8}-x_{2}\right) \\
\dot{x}_{3}=-\beta x_{2} \\
\dot{x}_{4}=\alpha\left(x_{5}-f_{2}\left(x_{4}\right)\right) \\
\dot{x}_{5}=x_{4}-x_{5}+x_{6}+h\left(x_{2}-x_{5}\right) \\
\dot{x}_{6}=-\beta x_{5}, \\
\dot{x}_{7}=\alpha\left(x_{8}-f_{3}\left(x_{7}\right)\right) \\
\dot{x}_{8}=x_{7}-x_{8}+x_{9}+h\left(x_{5}-x_{8}\right) \\
\dot{x}_{9}=-\beta x_{8},
\end{array}\right.
$$

where

$$
f_{1}\left(x_{1}\right)= \begin{cases}\frac{b \pi}{2 a}\left(x_{1}-2 a c_{1}\right) & x_{1} \geq 2 a c_{1} \\ -b \sin \left(\frac{\pi x_{1}}{2 a}+d_{1}\right) & -2 a c_{1}<x_{1}<2 a c_{1} \\ \frac{b \pi}{2 a}\left(x_{1}+2 a c_{1}\right) & x_{1} \leq-2 a c_{1},\end{cases}
$$

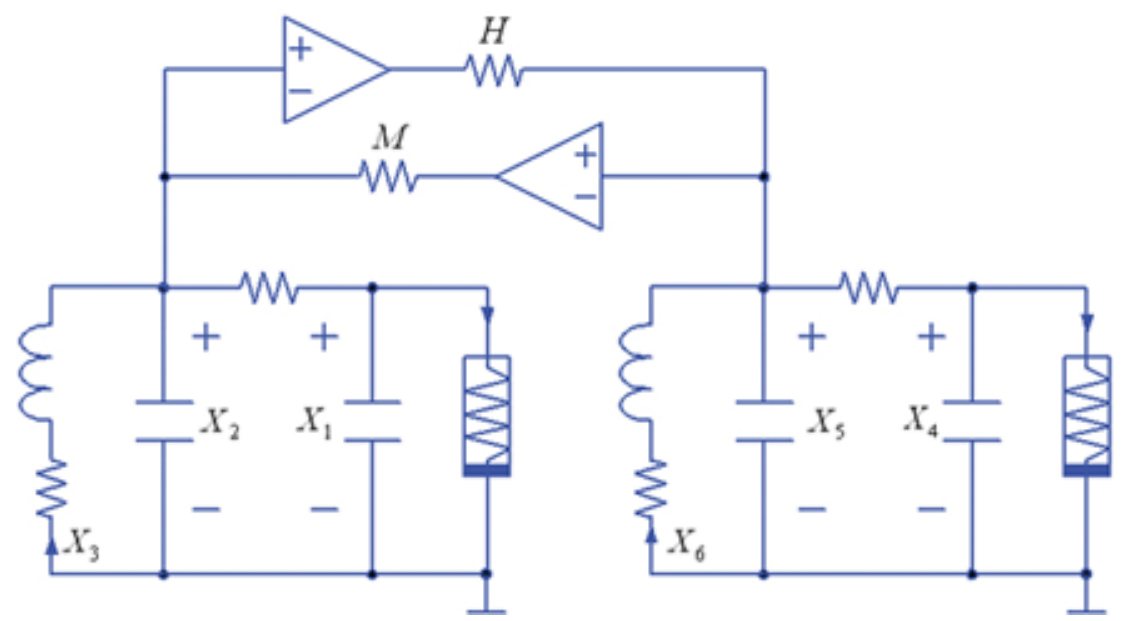

Fig. 68. Two-coupled Chua's circuit with nonlinear sine resistors. 


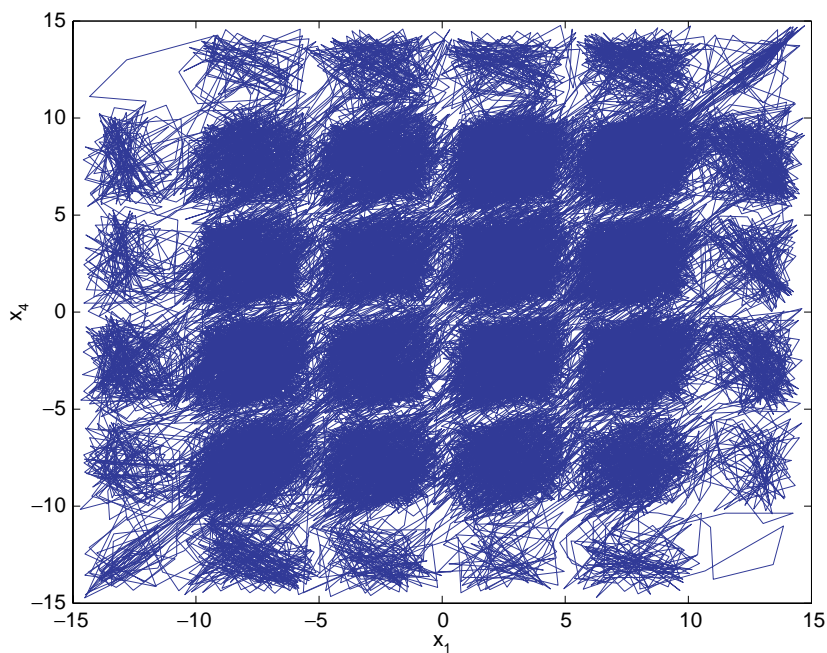

(a) 2-D $6 \times 6$-grid scroll attractor

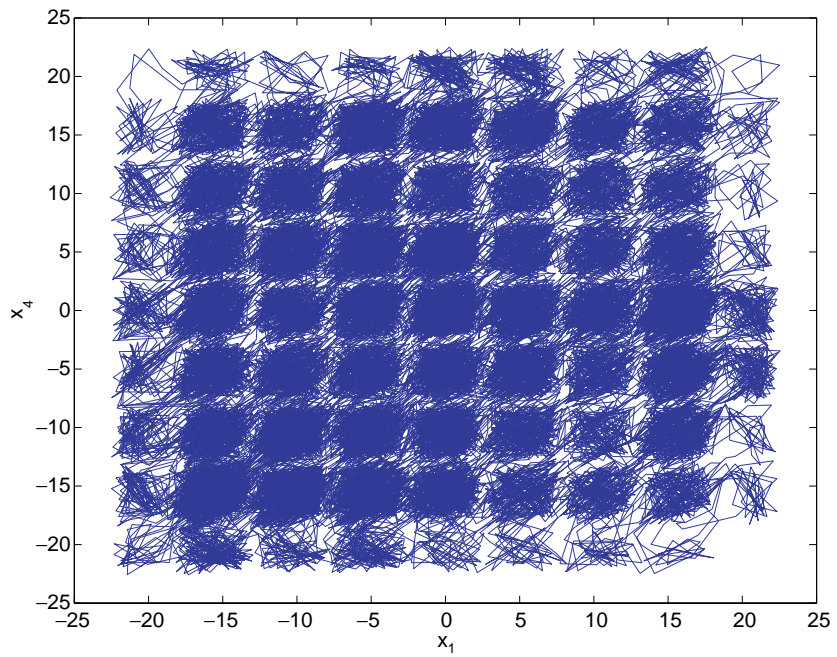

(b) 2 -D $9 \times 9$-grid scroll attractor

Fig. 69. Numerical simulations of 2-D $m \times n$-grid scroll hyperchaotic attractors.

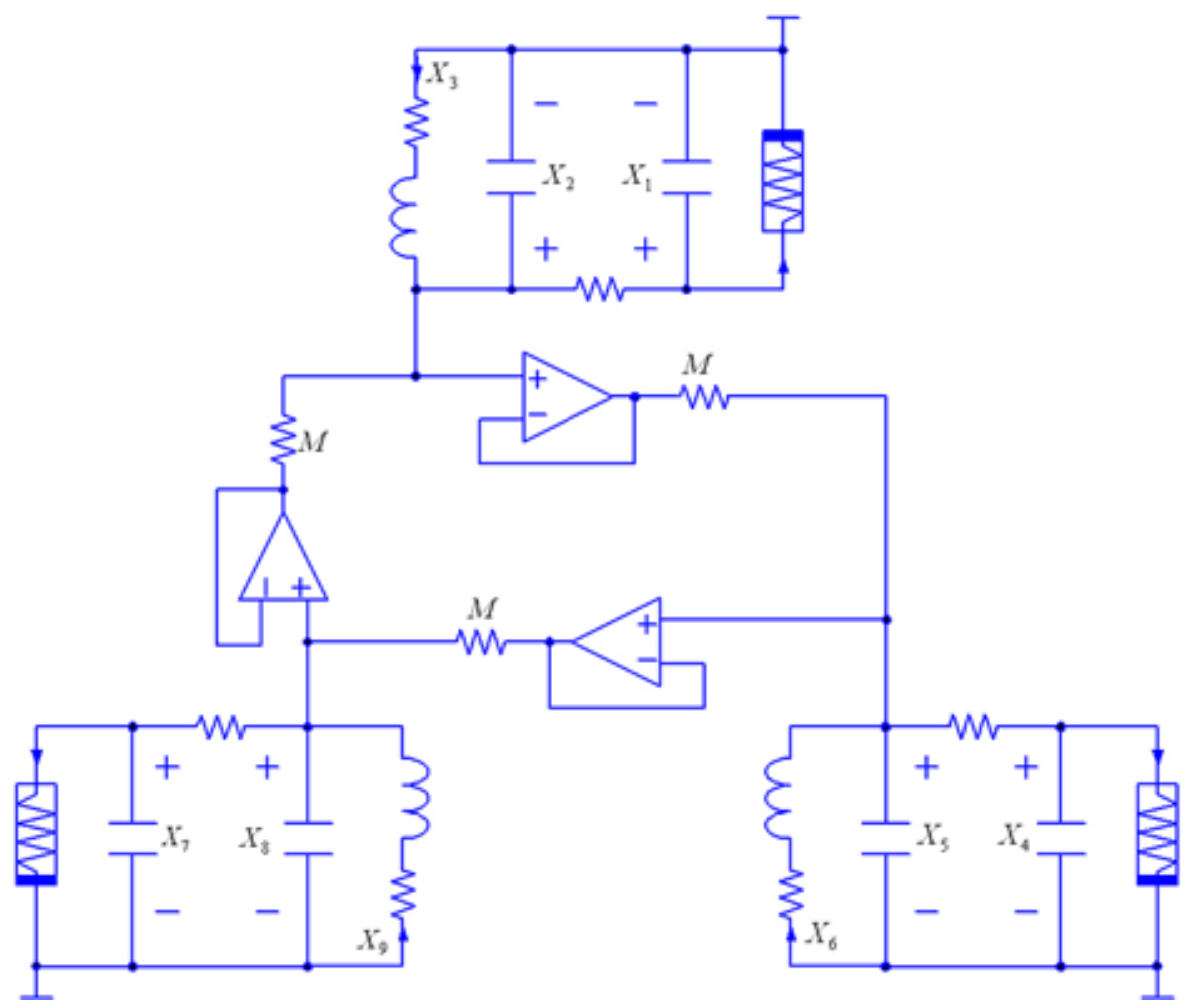

Fig. 70. Three-coupled Chua's circuit forming a ring with nonlinear sine resistors.

$f_{2}\left(x_{4}\right)= \begin{cases}\frac{b \pi}{2 a}\left(x_{4}-2 a c_{2}\right) & x_{4} \geq 2 a c_{2} \\ -b \sin \left(\frac{\pi x_{4}}{2 a}+d_{2}\right) & -2 a c_{2}<x_{4}<2 a c_{2} \\ \frac{b \pi}{2 a}\left(x_{4}+2 a c_{2}\right) & x_{4} \leq-2 a c_{2},\end{cases}$

$f_{3}\left(x_{7}\right)= \begin{cases}\frac{b \pi}{2 a}\left(x_{7}-2 a c_{3}\right) & x_{7} \geq 2 a c_{3} \\ -b \sin \left(\frac{\pi x_{7}}{2 a}+d_{3}\right) & -2 a c_{3}<x_{7}<2 a c_{3} \\ \frac{b \pi}{2 a}\left(x_{7}+2 a c_{3}\right) & x_{7} \leq-2 a c_{3} .\end{cases}$ 


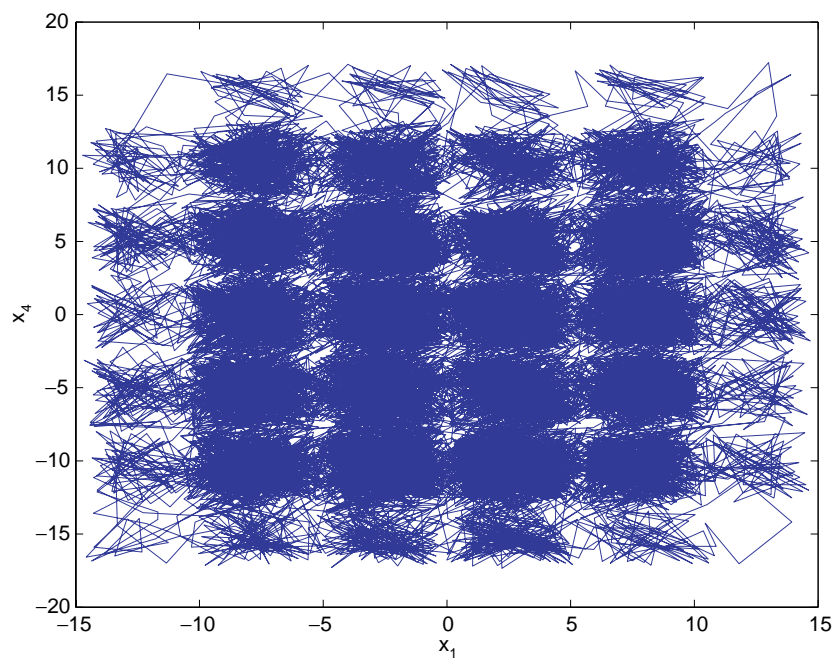

(a) $x_{1}-x_{4}$ plane projection

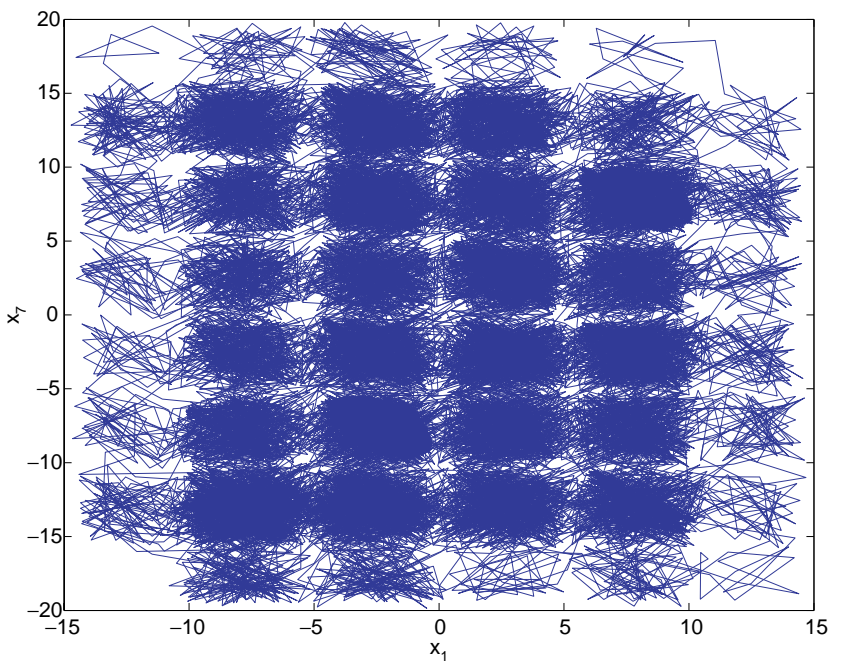

(b) $x_{1}-x_{7}$ plane projection

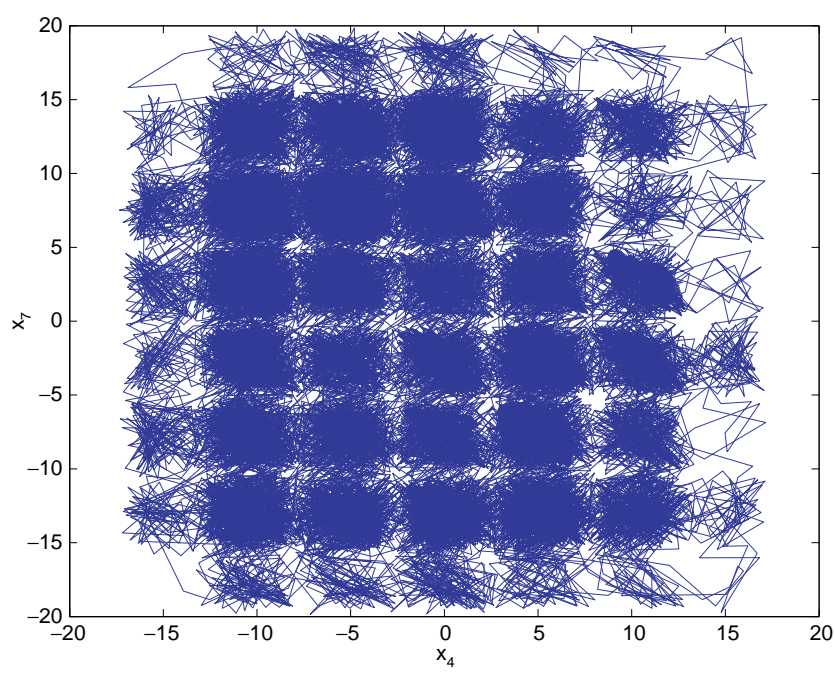

(c) $x_{4}-x_{7}$ plane projection

Fig. 71. 3-D $6 \times 7 \times 8$-grid scroll hyperchaotic attractor.

Here, $\alpha, \beta, a, b, c_{1}, c_{2}, c_{3}, d_{1}, d_{2}, d_{3}$ are real constants and $d_{i}(i=1,2,3)$ are defined by

$$
d_{i}= \begin{cases}\pi & \text { for } c_{i} \text { is even } \\ 0 & \text { for } c_{i} \text { is odd }\end{cases}
$$

Figure 70 displays the coupling structure of three Chua's circuits forming a ring with sine nonlinearities. Assume that $\alpha=10.814, \beta=14.0$, $a=1.3, b=0.11, h=0.18$ and $c_{1}, c_{2}, c_{3}, d_{1}$, $d_{2}, d_{3}$ are design parameters. System (63) can create 3 -D $m \times n \times l$-grid scroll hyperchaotic attractors for suitable design parameters. When $c_{1}=5$, $c_{2}=6, c_{3}=7, d_{1}=d_{3}=0, d_{2}=\pi$, system (63) has a 3 -D $6 \times 7 \times 8$-grid scroll hyperchaotic attractor, as shown in Fig. 71 . For the $6 \times 7 \times 8$-grid scroll attractor, the Lyapunov exponent spectrum is $L E_{1}=0.675, L E_{2}=0.297, L E_{3}=0.180, L E_{4}=0$, $L E_{5}=-0.127, L E_{6}=-0.718, L E_{7}=-1.078$, $L E_{8}=-1.302, L E_{9}=-1.518$.

\section{Design of Multiscroll Chaotic Attractors via Other Techniques}

In this section, other techniques for generating multiscroll chaotic attractors are reviewed, including the modified Lorenz system, the $n$th covers of the proto-Lorenz system [Miranda \& Stone, 1993], a critical chaotic system, and several nonautonomous continuous dynamical systems. 


\subsection{Multiscroll chaotic attractors from the modified Lorenz system}

This subsection discusses a modified Lorenz system [Elwakil et al., 2002; Lü et al., 2002c; Chen \& Lü, 2003], which can create a four-wing butterfly chaotic attractor.

The classical Lorenz system [Chen \& Lü, 2003] is described by

$$
\left\{\begin{array}{l}
\dot{x}=a(y-x) \\
\dot{y}=c x-y-x z \\
\dot{z}=x y-b z
\end{array}\right.
$$

where $a, b, c$ are constants and the nonlinear terms $x z, x y$ are responsible for chaos generation.

Elwakil et al. [2002] presented a novel Lorenztype system, which is free from the positive $z$ constraint, given by

$$
\left\{\begin{array}{l}
\dot{x}=a(y-x) \\
\dot{y}_{\mp}=\mp K z \\
\dot{z}_{ \pm}= \pm|x| \mp 1,
\end{array}\right.
$$

where

$$
K= \begin{cases}1, & x \geq 0 \\ -1, & x<0\end{cases}
$$

Here, $0<a<1$. Comparing with the original Lorenz system (64), the nonlinear terms $x y, x z$ have been replaced with the absolute value function $|x|$ and the sign function $K z$, respectively. Moreover, the threshold constant $b$ and the damping constant $c$ have been removed in system (65).

System (65) is a dual system with two complementary modes of operation. Let $S(-,+)$ denote the case of $\dot{y}=\dot{y}_{-}, \dot{z}=\dot{z}_{+}$and $S(+,-)$ denote the case of $\dot{y}=\dot{y}_{+}, \dot{z}=\dot{z}_{-}$. Note that the threshold effect performed by $b$ in (64) is now performed by $|x|$ in (65). Furthermore, in (65), it is not the sign of $(b-z)$ but the sign of $z$ which changes at the threshold, ultimately removing the constraint inherited within the Lorenz system.

When $a=0.55$, system (65) with the two modes $S(-,+)$ and $S(+,-)$ can generate two-wing butterfly attractors similar to the original Lorenz system (64). Because the eigenvalue pattern is independent of the mode in which the system operates, one can utilize an external source to force switching to occur between $S(-,+)$ and $S(+,-)$. Figure $72(\mathrm{a})$ shows the four-wing butterfly chaotic attractor generated in this case, where $a=0.55$ and a pulse train with period $T_{F}=250 T_{S}$ is used to drive the switching.

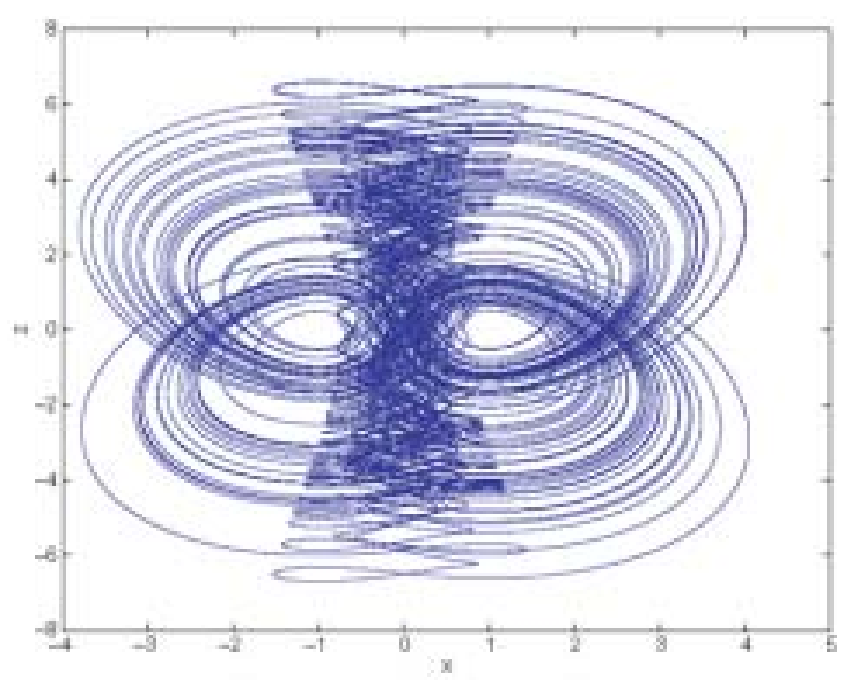

(a) Numerical simulation of the four-wing butterfly chaotic attractor

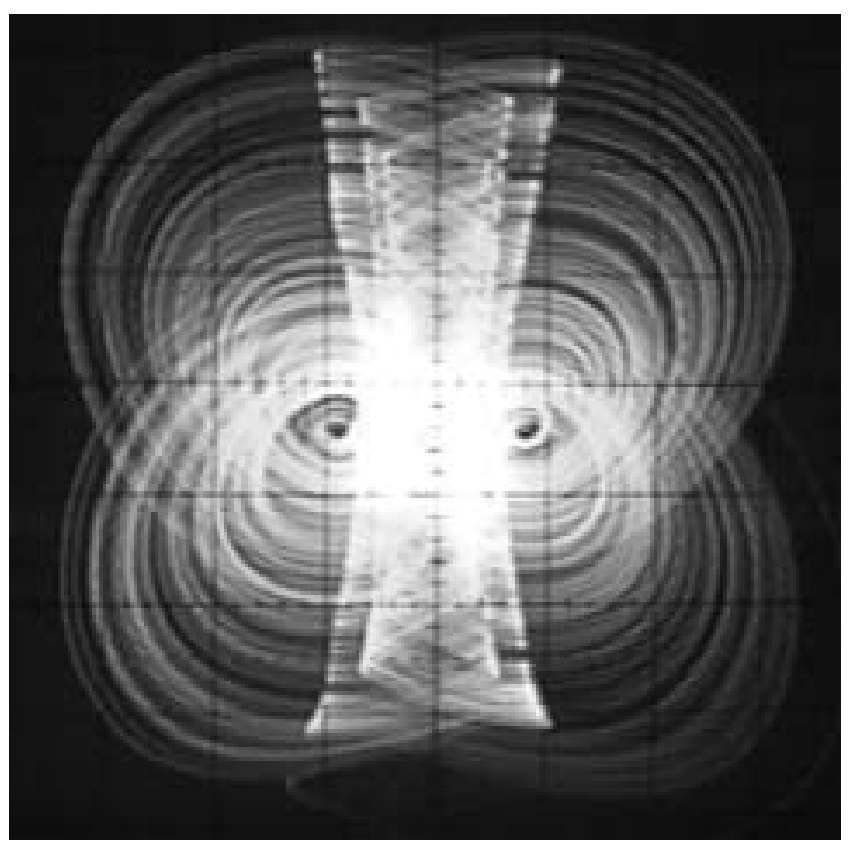

(b) Experimental observation of the four-wing butterfly chaotic attractor [Elwakil et al., 2002]

Fig. 72. Four-wing butterfly chaotic attractors from the modified Lorenz system.

Elwakil et al. [2002] also designed a circuit diagram to experimentally verify the four-wing butterfly chaotic attractor. Figure 73 [Elwakil et al., 2002] displays the circuit diagram. It mainly includes three capacitors $\left(C_{X}, C_{Y}, C_{Z}\right)$, twelve bilateral MOS analog switches, six current feedback operational amplifiers (CFOA), an external voltage source $V_{P}$, and a reference voltage $V_{R}$. 


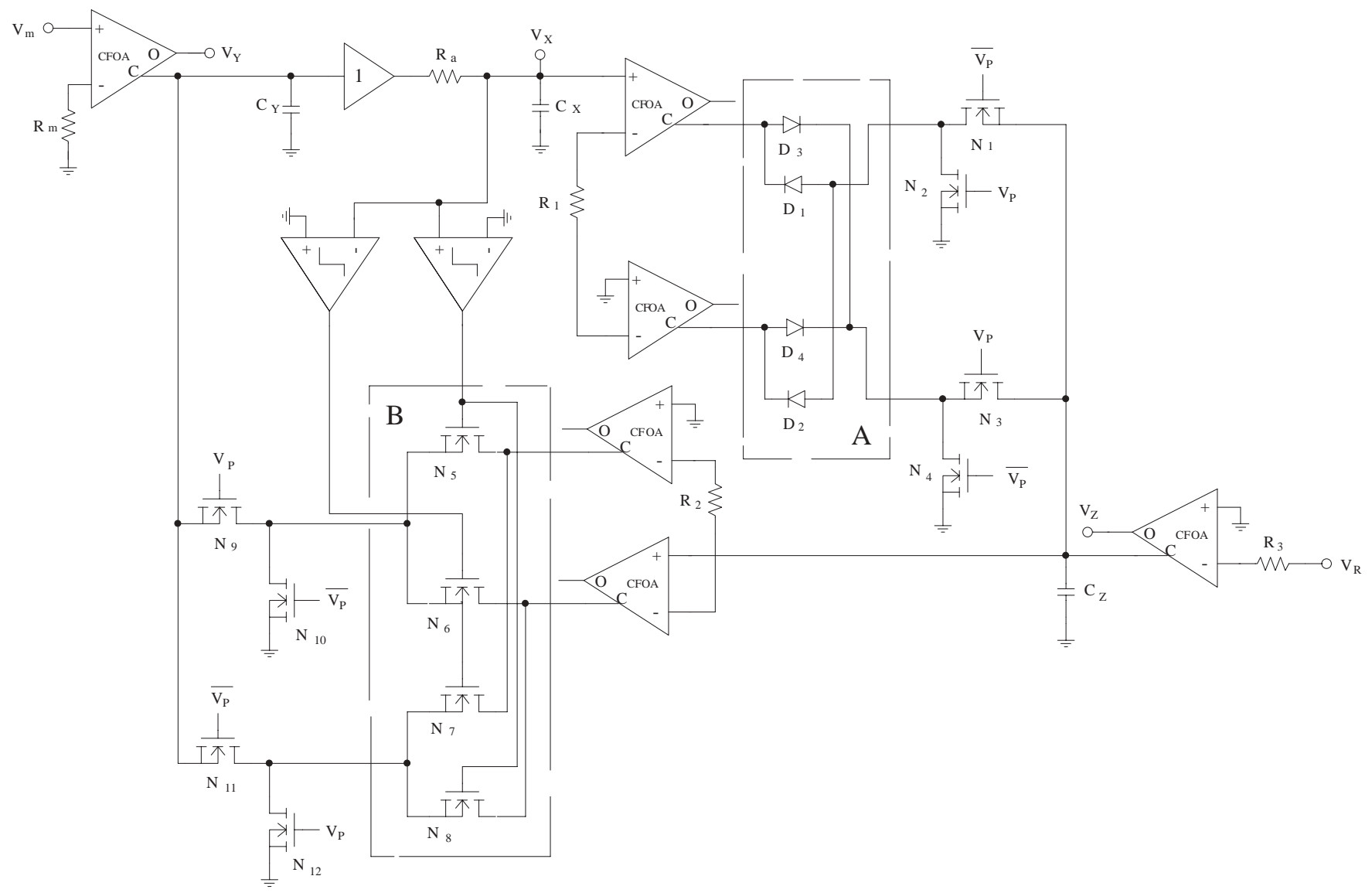

Fig. 73. Circuit diagram for realization of the modified Lorenz system [Elwakil et al., 2002].

By selecting $C_{X}=C_{Y}=C_{Z}=C, R_{1}=$ $R_{2}=R_{3}=R, R_{a}=R / a$, defining $x=V_{X} / V_{R}$, $y=V_{Y} / V_{R}, z=V_{Z} / V_{R}$, and normalizing time with respect to $R C$, it is easy to verify that the circuit diagram shown in Fig. 73 indeed realizes system (65). Figure 72(b) [Elwakil et al., 2002] shows the experimental observation of the four-wing butterfly chaotic attractor, where the frequency of the source is $150 \mathrm{HZ}$ and the center frequency of the circuit is approximately $31 \mathrm{kHZ}$.

\subsection{Multiscroll chaotic attractors from the modified proto-Lorenz system}

This subsection briefly introduces the so-called proto-Lorenz system [Miranda \& Stone, 1993], which is a quotient of the Lorenz system. Based on the proto-Lorenz system, one can design and generate $n$-scroll chaotic attractors.
Recall the Lorenz system (64), where $(x, y, z) \in$ $X=\mathbf{R}^{3}$. Obviously, the Lorenz system (64) is invariant under the linear transformation $L_{1}:(-x$, $-y, z) \rightarrow(x, y, z)$.

Define a map, $\pi: X \rightarrow Y$, as follows:

$$
\begin{gathered}
u=\pi(x)=x^{2}-y^{2}, \quad v=\pi(y)=2 x y, \\
z=\pi(z)=z .
\end{gathered}
$$

Since the Lorenz system (64) is invariant in $X$ under the transformation $L_{1}$, it descends to a vector field $\mathcal{F}$ on the quotient $Y$. Miranda and Stone [1993] derived the equation of this descended vector field $\mathcal{F}$ on the orbit space $Y$, as follows:

$$
\left\{\begin{aligned}
\dot{u}= & -(a+1) u+(a-c+z) v \\
& +(1-c) \sqrt{u^{2}+v^{2}} \\
\dot{v}= & (c-a) u-(a+1) v \\
& +(a+c-z) \sqrt{u^{2}+v^{2}}-u z \\
\dot{z}= & \frac{1}{2} v-b z,
\end{aligned}\right.
$$




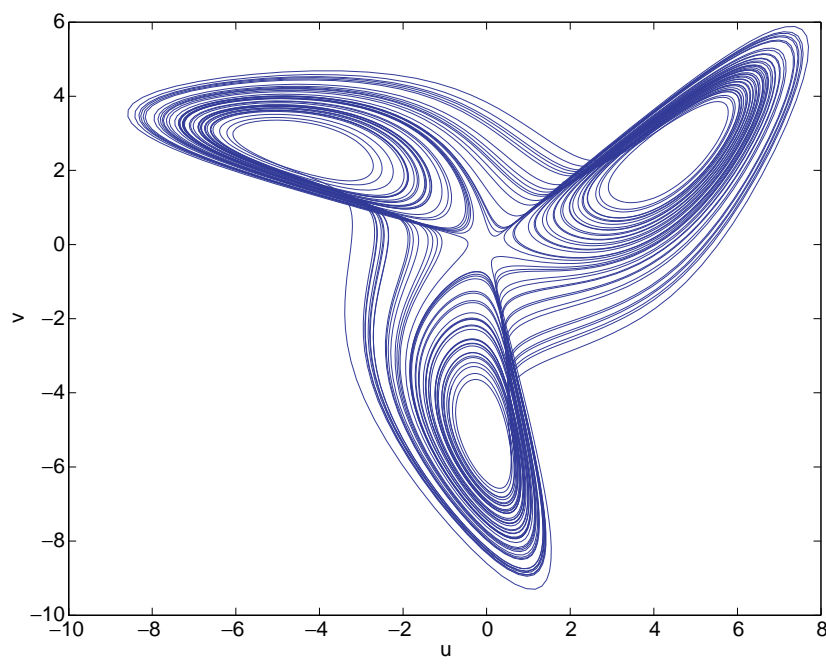

(a) 3-Scroll chaotic attractor

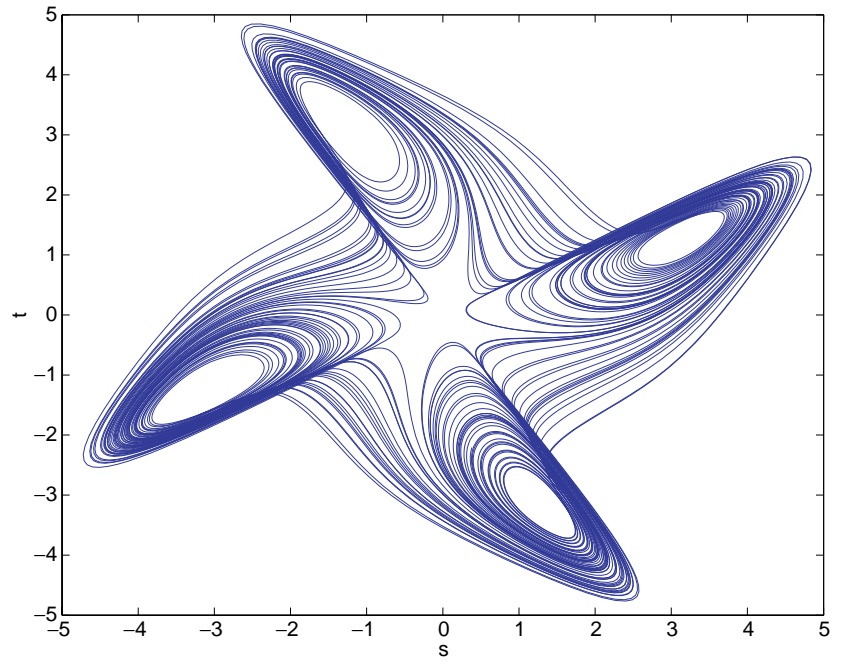

(b) 4-Scroll chaotic attractor

Fig. 74. Multiscroll chaotic attractors from the modified proto-Lorenz system.

which is called the proto-Lorenz system. It is noticed that the proto-Lorenz system $\mathcal{F}$ is a continuous vector field on all of $Y$. However, it is not differentiable because of the presence of the norm $\sqrt{u^{2}+v^{2}}$. Of course, it is differentiable away from the $z$-axis.

According to the construction of the protoLorenz system, the double cover $\mathcal{F}_{2}$ of this system is the original Lorenz system. Moreover, the $n$th cover $\mathcal{F}_{n}$ of the proto-Lorenz system has $n$ scrolls. The triple cover $\mathcal{F}_{3}$ [Miranda \& Stone, 1993] is described by

$$
\left\{\begin{aligned}
\dot{p}= & \frac{1}{3}[-(a+1) p+(a-c+z) q] \\
& +\frac{1}{3 \sqrt{p^{2}+q^{2}}}\left[(1-a)\left(p^{2}-q^{2}\right)\right. \\
& +2(a+c-z) p q] \\
\dot{q}= & \frac{1}{3}[(c-a-z) p-(a+1) q] \\
& +\frac{1}{3 \sqrt{p^{2}+q^{2}}}[2(a-1) p q \\
& \left.+(a+c-z)\left(p^{2}-q^{2}\right)\right] \\
\dot{z}= & \frac{1}{2}\left(3 p^{2} q-q^{3}\right)-b z .
\end{aligned}\right.
$$

When $a=10, b=8 / 3, c=28$, the triple-cover system (68) has a 3 -scroll chaotic attractor, as shown in Fig. 74(a).
Similarly, the quartic cover $\mathcal{F}_{4}$ [Miranda \& Stone, 1993] is given by

$$
\left\{\begin{aligned}
\dot{s}= & \frac{1}{2\left(s^{2}+t^{2}\right)}\left[-a s^{3}+(2 a+c-z) s^{2} t\right. \\
& \left.+(a-2) s t^{2}-(c-z) t^{3}\right] \\
\dot{t}= & \frac{1}{2\left(s^{2}+t^{2}\right)}\left[(c-z) s^{3}+(a-2) s^{2} t\right. \\
& \left.+(-2 a-c+z) s t^{2}-a t^{3}\right] \\
\dot{z}= & 2 s^{3} t-2 s t^{3}-b z .
\end{aligned}\right.
$$

When $a=10, b=8 / 3, c=28$, the quartic-cover system (69) displays a 4 -scroll chaotic attractor, as shown in Fig. 74(b).

\subsection{Multiscroll chaotic attractors from a critical chaotic system}

This subsection presents a 3D quadratic autonomous critical chaotic system, which can display (i) two 1-scroll chaotic attractors simultaneously, with only three equilibria, and (ii) two 2-scroll chaotic attractors simultaneously, with five equilibria [Lü \& Chen, 2002; Lü et al., 2002d, 2004a].

Lü et al. [2004a] introduced the following critical chaotic system:

$$
\left\{\begin{array}{l}
\dot{x}=-\frac{a b}{a+b} x-y z+c \\
\dot{y}=a y+x z \\
\dot{z}=b z+x y
\end{array}\right.
$$

where $a, b, c$ are real constants. 


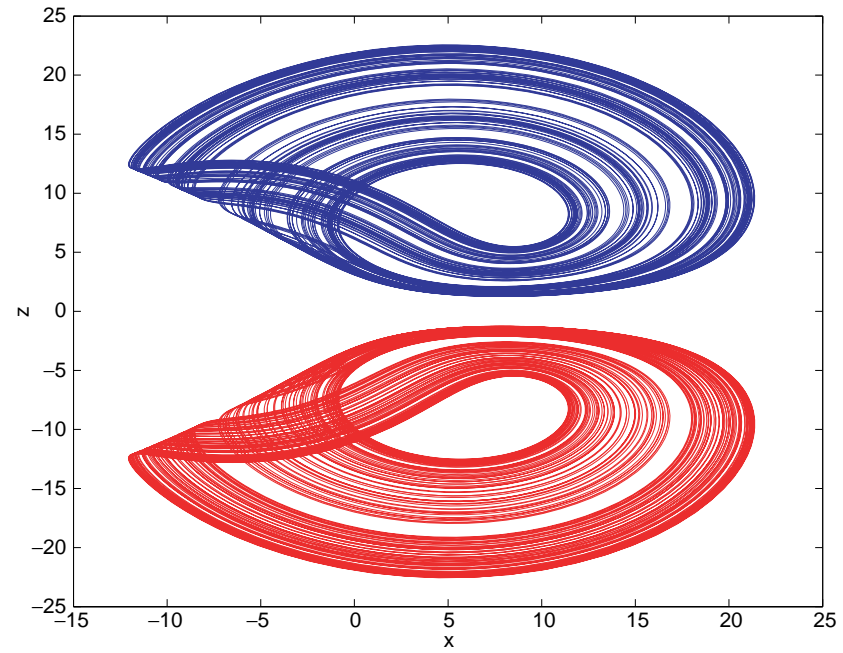

(a) Two coexisting 1-scroll chaotic attractors

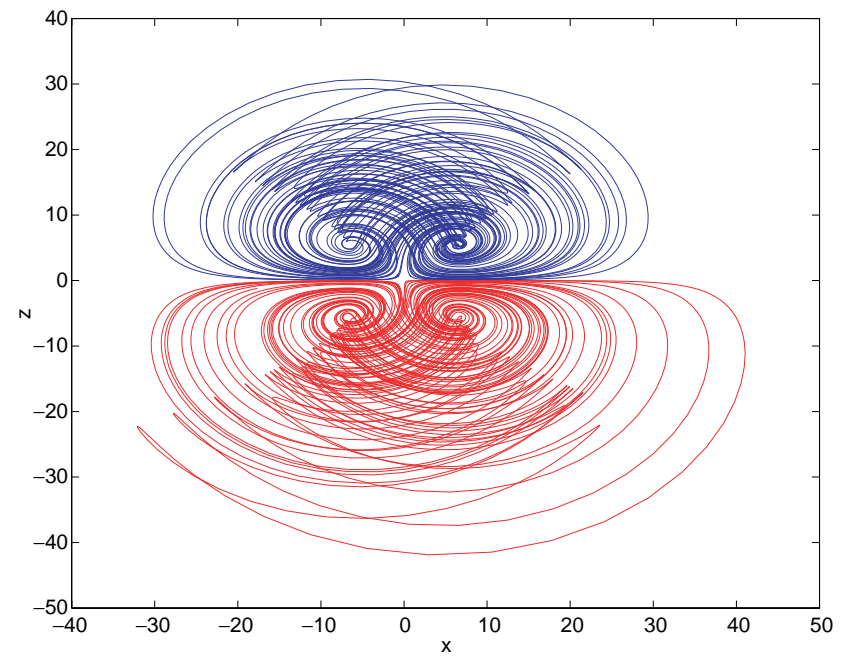

(b) Two coexisting 2-scroll chaotic attractors

Fig. 75. Multiscroll chaotic attractors from the critical chaotic system.

System (70) is found to be chaotic in a wide parameter range and has many interesting complex dynamical behaviors [Lü et al., 2004a]. Especially, when $a=-10, b=-4, c=18.1$, it displays two coexisting 1 -scroll chaotic attractors, as shown in Fig. 75(a). The corresponding Lyapunov exponent spectrum of system (70) is $\lambda_{1}=0.253223, \lambda_{2}=0, \lambda_{3}=-11.3944$, and the Lyapunov dimension is $d_{L}=2.0221$ for initial value $(1,1,1)$. Moreover, when $a=-10, b=-4, c=0$, system (70) displays two coexisting 2-scroll chaotic attractors, as shown in Fig. 75(b). Similarly, the corresponding Lyapunov exponent spectrum of system $(70)$ is $\lambda_{1}=1.1662, \lambda_{2}=0, \lambda_{3}=-12.3090$, and the Lyapunov dimension is $d_{L}=2.0947$ for initial value $(1,1,1)$. It should be pointed out that Liu \& Chen [2004] also proposed a similar but different chaotic system, which can create two double-scroll chaotic attractors that are extremely closely located seemingly just like a four-scroll attractor.

\subsection{Multiscroll chaotic and nonchaotic attractors from the quasi-periodically forced system}

This subsection introduces a general quasiperiodically forced system, which can generate multiscroll chaotic and nonchaotic attractors.

Kapitaniak [Aziz-Alaoui, 1999, 2000] once investigated the dynamics of a nonautonomous circuit, which is a classical forced negative-resistance oscillator and can generate a strange nonchaotic two-frequency torus attractors. The circuit equation is described by

$$
\left\{\begin{array}{l}
\dot{x}=y-f_{2}(x) \\
\dot{y}=-\beta\left[x+(v+1) y+A\left(\sin \left(\omega_{1} t\right)+\sin \left(\omega_{2} t\right)\right)\right]
\end{array}\right.
$$

where

$$
f_{2}(x)=m_{1} x+\frac{1}{2}\left(m_{0}-m_{1}\right)[|x+1|-|x-1|],
$$

and $\beta, v, \omega_{1}, \omega_{2}, m_{0}, m_{1}, A$ are parameters.

To generate multiscroll chaotic or nonchaotic attractors, Aziz-Alaoui [1999] replaced the function $f_{2}$ in (71) by the following function (13), obtaining

$$
\left\{\begin{array}{l}
\dot{x}=y-f_{N}(x) \\
\dot{y}=-\beta\left[x+(v+1) y+A\left(\sin \left(\omega_{1} t\right)+\sin \left(\omega_{2} t\right)\right)\right] .
\end{array}\right.
$$

Obviously, the parameters set satisfies

$$
\mathcal{B}_{N}^{K}=\left\{\beta, v, \omega_{1}, \omega_{2}, A\right\} \cup \mathcal{B}_{N} \subset \mathbf{R}^{2 N+4},
$$

where $\mathcal{B}_{N}=\left\{\left(s_{k}\right)_{k \in I_{N-2}},\left(m_{k}\right)_{k \in I_{N-1}}\right\} \subset \mathbf{R}^{2 N-1}$. Let $m_{0}=m_{2 i}=\cdots=-1.02$ and $m_{1}=m_{2 i+1}=$ $\cdots=-0.55$ for $i=1,2, \ldots$.

Figure 76(a) [Aziz-Alaoui, 1999] shows two coexisting 3-scroll chaotic attractors for $N=6, A=$ 0.173, $\mathcal{B}_{6}^{K}$; Fig. 76(b) [Aziz-Alaoui, 1999] displays the combination of these two chaotic attractors with the formation of a double 3-scroll chaotic attractor 


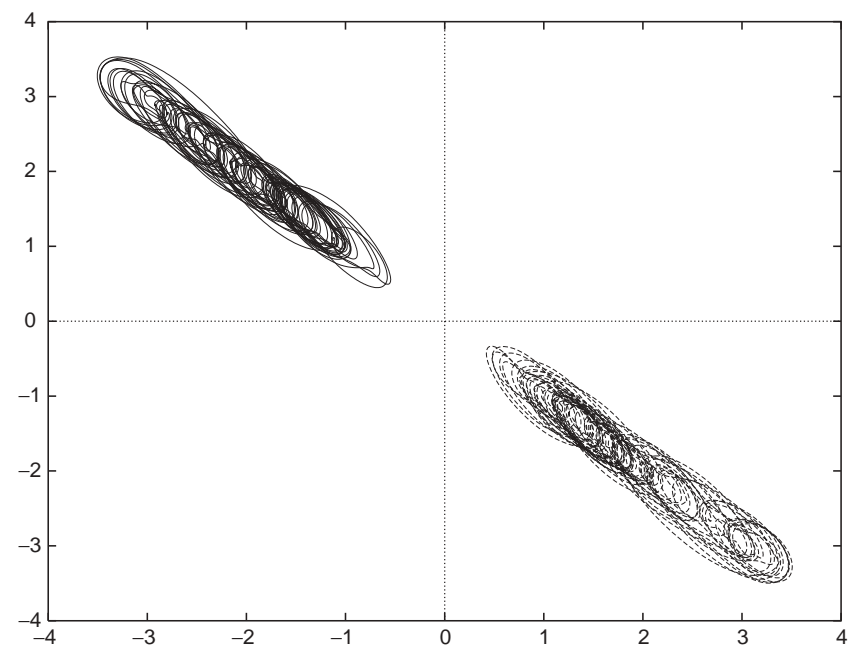

(a) Two coexisting 3 -scroll attractors for $A=0.173$

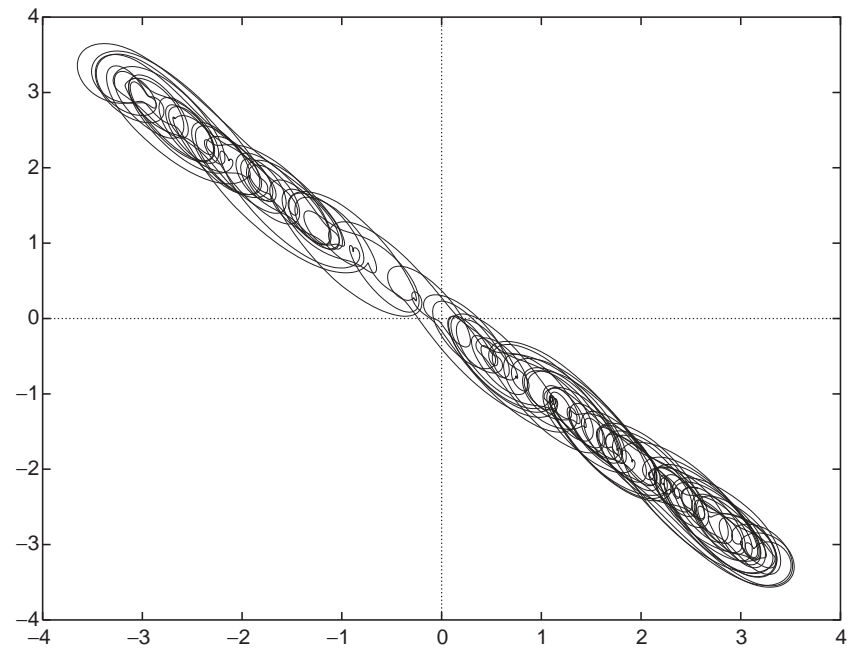

(b) A double 3-scroll attractor for $A=0.18$

Fig. 76. Multiscroll chaotic attractors from the quasi-periodically forced system [Aziz-Alaoui, 1999].

for $N=6, A=0.18, \mathcal{B}_{6}^{K}$, where $\mathcal{B}_{6}^{K}=\{\beta=1.0$, $v=0.015, \omega_{1}=0.75, \omega_{2}=\sqrt{2}, m_{0}=-1.02$, $m_{2}=-0.55, s_{0}=1.0, s_{1}=1.11, s_{2}=2.06$, $\left.s_{3}=2.14, s_{4}=3.06\right\} \cup\{A\}$.

\subsection{Multiscroll strange attractors in the PWL van der Pol system}

This subsection presents a general van der Pol system with periodic forcing [Aziz-Alaoui, 1999], which can create multiscroll strange attractors.

This van der Pol system with periodic forcing is described by

$$
\left\{\begin{array}{l}
\varepsilon \frac{d x}{d t}=y-\left(\frac{x^{2}}{3}-x\right) \\
\frac{d y}{d t}=-\alpha x+b \cos (t),
\end{array}\right.
$$

where $\alpha, \varepsilon$ are sufficiently small and $b>0$. The associated PWL system of (74) [Aziz-Alaoui, 1999] is

$$
\left\{\begin{array}{l}
\varepsilon \frac{d x}{d t}=y-f_{2}(x) \\
\frac{d y}{d t}=-\alpha x+b \cos (t),
\end{array}\right.
$$

where $f_{2}(x)$ is defined by (72) with $m_{0}=-1$, $m_{1}=1$. When $\alpha=\varepsilon=0.167$ and $b=0.59983$, system (74) exhibits a positive Lyapunov exponent.
However, the range of the parameter $b$ corresponding to this attractor is very narrow. Moreover, this attractor is easily destroyed by a tiny perturbation.

To create $n$-scroll strange attractors, AzizAlaoui [1999] modified system (75) as follows:

$$
\left\{\begin{array}{l}
\varepsilon \frac{d x}{d t}=y-f_{N}(x) \\
\frac{d y}{d t}=-\alpha x+b \cos (t),
\end{array}\right.
$$

where $f_{N}(x)$ is defined by (13). Clearly, the parameters set satisfies

$$
\mathcal{B}_{N}^{V}=\{\alpha, \varepsilon, b\} \cup \mathcal{B}_{N} \subset \mathbf{R}^{2 N+2},
$$

where $\mathcal{B}_{N}=\left\{\left(s_{k}\right)_{k \in I_{N-2}},\left(m_{k}\right)_{k \in I_{N-1}}\right\} \subset \mathbf{R}^{2 N-1}$. For simplicity, assume that $m_{0}=m_{2 i}=\cdots=-1.0$ and $m_{1}=m_{2 i+1}=\cdots=1.0$ for $i=1,2, \ldots$.

Figure 77(a) [Aziz-Alaoui, 1999] displays a strange attractor for $N=6$ and $s_{1}=2.7, s_{2}=$ 3.0482, $s_{3}=4.028, s_{4}=4.975$; Fig. 77(b) [AzizAlaoui, 1999] shows a strange attractor for $N=8$ and $s_{1}=2.7, s_{2}=3.0482, s_{3}=4.028, s_{4}=4.975$, $s_{5}=5.2, s_{6}=6.1$.

\subsection{Multiscroll chaotic attractors in the PWL-Duffing system}

This subsection describes a general PWL-Duffing system for generating multiscroll chaotic attractors. 


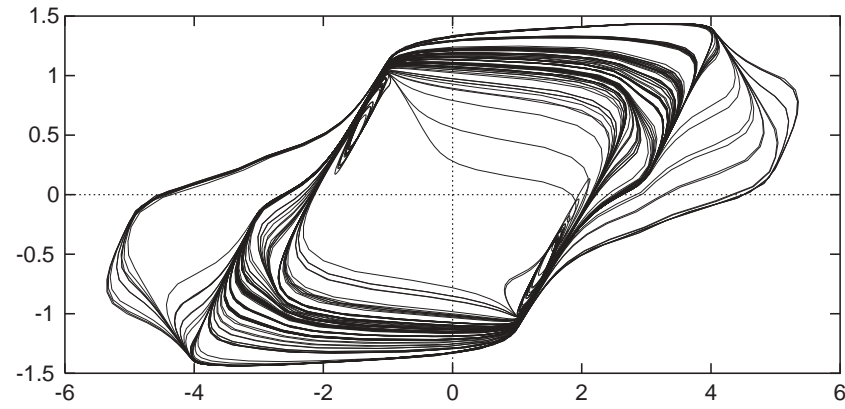

(a) A strange attractors for $N=6$

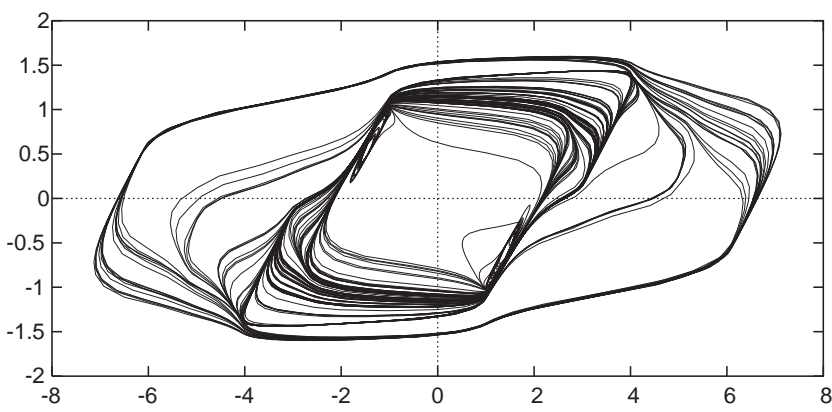

(b) A strange attractor for $N=8$

Fig. 77. Multiscroll strange attractors from the general van der Pol system [Aziz-Alaoui, 1999].

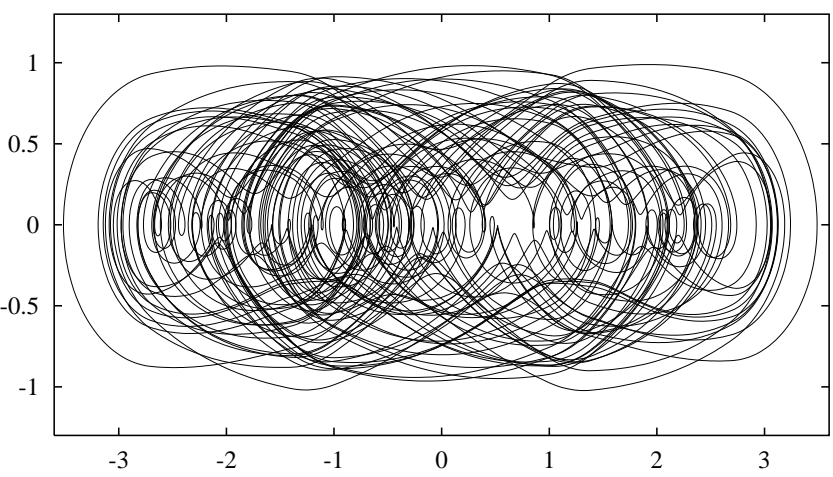

(a) 4-Scroll chaotic attractor

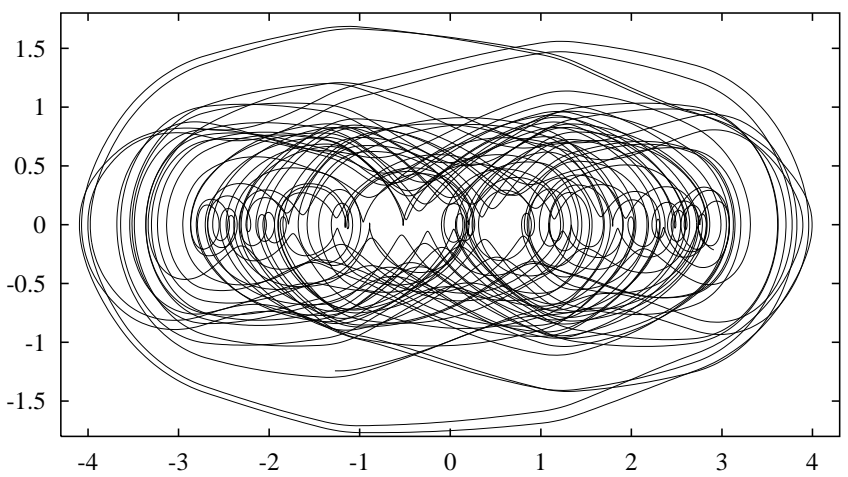

(b) 6-Scroll chaotic attractor

Fig. 78. Multiscroll chaotic attractors from the PWL-Duffing system [Aziz-Alaoui, 2000].

The classical Duffing system is described by

$$
\left\{\begin{array}{l}
\dot{x}=y \\
\dot{y}=x-x^{3}-\varepsilon y+\gamma \cos (\omega t),
\end{array}\right.
$$

where $x, y$ are the functions of time $t$. When $\varepsilon=$ $0.25, \gamma=0.3, \omega=1.0$, system (77) has a chaotic attractor.

The corresponding simplest PWL-Duffing system [Aziz-Alaoui, 2000], obtained by replacing $x^{3}-x$ by $f_{2}(x)$, is

$$
\left\{\begin{array}{l}
\dot{x}=y \\
\dot{y}=-f_{2}(x)-\varepsilon y+\gamma \cos (\omega t),
\end{array}\right.
$$

where $f_{2}(x)$ is defined by (72). When $\varepsilon=0.25$, $\gamma=0.3, \omega=1.0, m_{0}=-0.0845, m_{1}=0.66$, system (77) has a chaotic attractor.

To create $n$-scroll chaotic attractors, AzizAlaoui [2000] extended system (78) as follows:

$$
\left\{\begin{array}{l}
\dot{x}=y \\
\dot{y}=-f_{N}(x)-\varepsilon y+\gamma \cos (\omega t),
\end{array}\right.
$$

where $f_{N}(x)$ is defined by (13).
Figure 78(a) [Aziz-Alaoui, 2000] displays a 4scroll chaotic attractor, where $\varepsilon=0.25, \gamma=0.3$, $\omega=1.0, m_{0}=m_{2}=-0.0845, m_{1}=m_{3}=0.66$, $s_{1}=1.4, s_{2}=2.7$. Figure 78(b) [Aziz-Alaoui, 2000] shows a 6 -scroll chaotic attractor, where $\varepsilon=0.25$, $\gamma=0.3, \omega=1.0, m_{0}=m_{2}=m_{4}=-0.0845$, $m_{1}=m_{3}=m_{5}=0.66, s_{1}=1.4, s_{2}=2.7$, $s_{3}=3.67, s_{4}=4.5$.

\subsection{Multiscroll chaotic attractors in a nonautonomous chaotic system}

This subsection introduces a simple nonautonomous chaotic system for creating $n$-scroll chaotic attractors.

Aziz-Alaoui [1999] investigated the following nonautonomous circuit:

$$
\left\{\begin{array}{l}
C \frac{d v_{C}}{d \tau}=i_{L}-g\left(v_{C}\right) \\
L \frac{d i_{L}}{d \tau}=-R i_{L}-i_{C}+V_{S} \sin (\omega \tau),
\end{array}\right.
$$




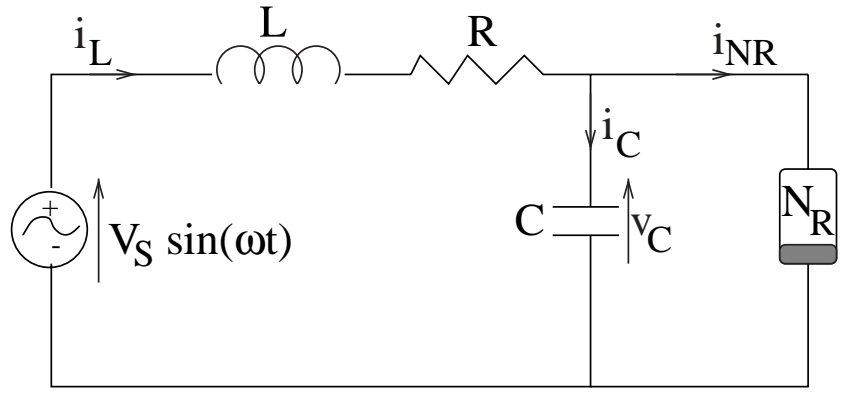

Fig. 79. Circuit diagram of the $n$-scroll nonautonomous circuit [Aziz-Alaoui, 1999].

where $g\left(v_{C}\right)$ is a PWL function defined by (2). The circuit diagram is shown in Fig. 79 [Aziz-Alaoui, 1999].

Let $x=v_{C} / b_{1}, y=i_{L} R / b_{1}, t=\tau / R C$, $\Omega=\omega / 2 \pi R C, \alpha=C R^{2} / L, K=V_{S} / b_{1}$. Thus, the corresponding dimensionless state equations are described by

$$
\left\{\begin{array}{l}
\frac{d x}{d t}=y-f_{2}(x) \\
\frac{d y}{d t}=-\alpha(x+y)+\alpha K \sin (2 \pi \Omega t),
\end{array}\right.
$$

where $f_{2}(x)$ is defined by $(72)$.

To generate $n$-scroll chaotic attractors from (81), Aziz-Alaoui [1999] modified system (81) as

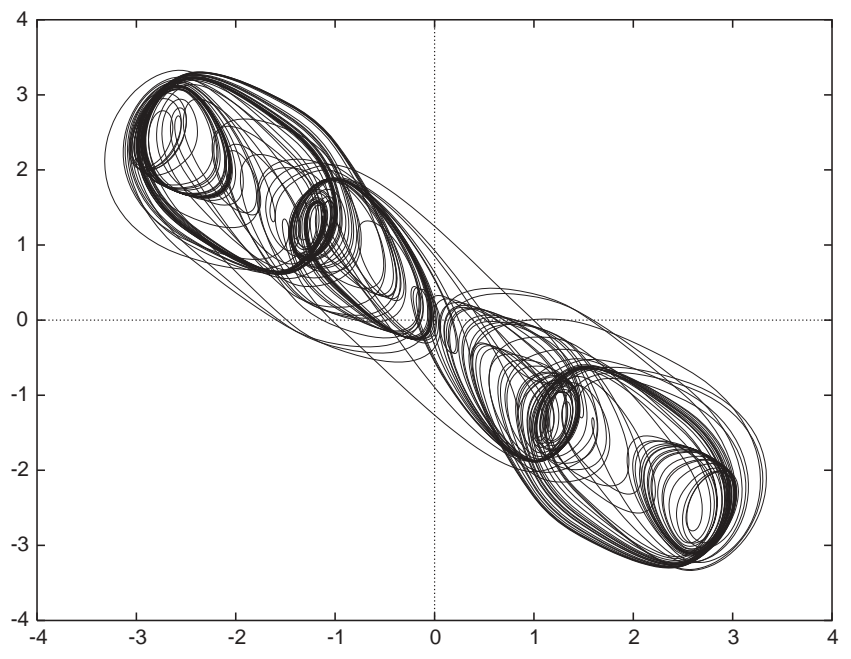

(a) 4-Scroll chaotic attractor follows:

$$
\left\{\begin{array}{l}
\frac{d x}{d t}=y-f_{N}(x) \\
\frac{d y}{d t}=-\alpha(x+y)+\alpha K \sin (2 \pi \Omega t),
\end{array}\right.
$$

where $f_{N}(x)$ is defined by (13). Aziz-Alaoui [1999] further investigated the equilibrium points and eigenspaces of this system (82).

When $N=4$ and $\mathcal{B}_{4}^{L}=\{\alpha=0.81, K=1.19$, $\Omega=0.22, m_{0}=-1.45, m_{1}=0.66, s_{1}=1.45$, $\left.s_{2}=2.49\right\}$, system (82) has a 4 -scroll chaotic attractor, as shown in Fig. 80(a) [Aziz-Alaoui, 1999]. When $N=6$ and $\mathcal{B}_{6}^{L}=\{\alpha=0.81, K=1.19$, $\Omega=0.22, m_{0}=-1.45, m_{1}=0.66, s_{1}=1.45$, $\left.s_{2}=2.49, s_{3}=2.94, s_{4}=4.1\right\}$, system (82) has a 6-scroll chaotic attractor, as shown in Fig. 80(b) [Aziz-Alaoui, 1999].

\subsection{Multiscroll chaotic attractors in a nonautonomous pulse-driven system}

In this subsection, a novel nonautonomous pulsedriven chaotic oscillator is discussed, which is based on the passive structure of Chua's circuit [Elwakil, 2002 .

It is well known that most nonautonomous chaotic circuits are driven by sinusoidal excitations. This implies that the equilibrium points of these driven systems are time-varying. Recently, Elwakil [2002] proposed a nonautonomous

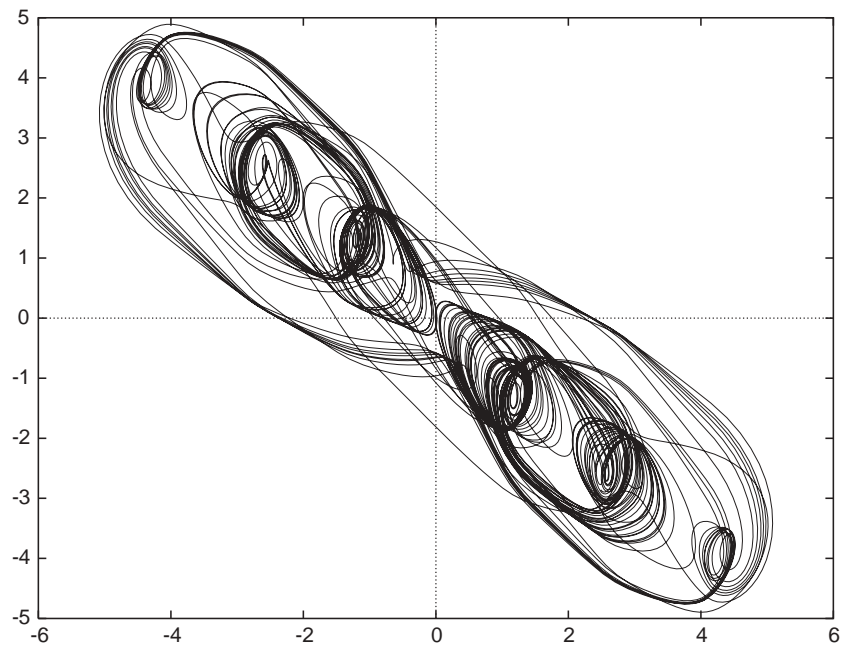

(b) 6-Scroll chaotic attractor

Fig. 80. $n$-Scroll chaotic attractors from the nonautonomous system [Aziz-Alaoui, 1999]. 


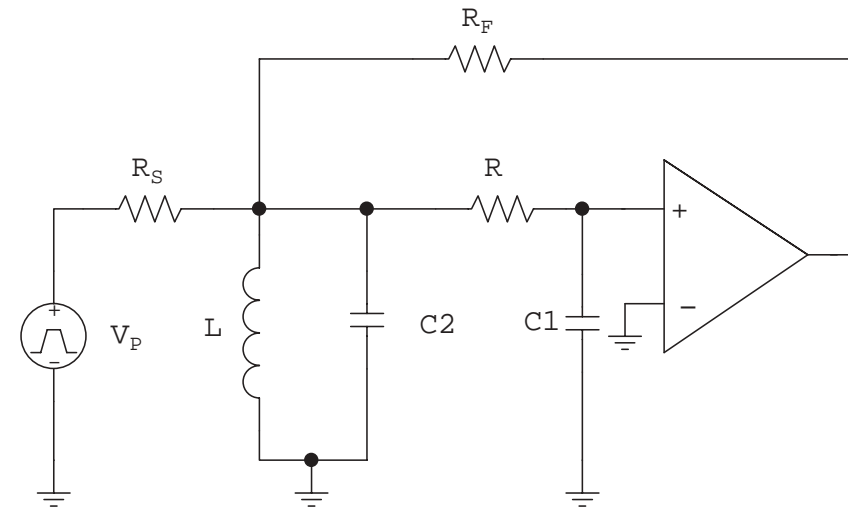

(a) Circuit structure with excitation across $C_{2}$

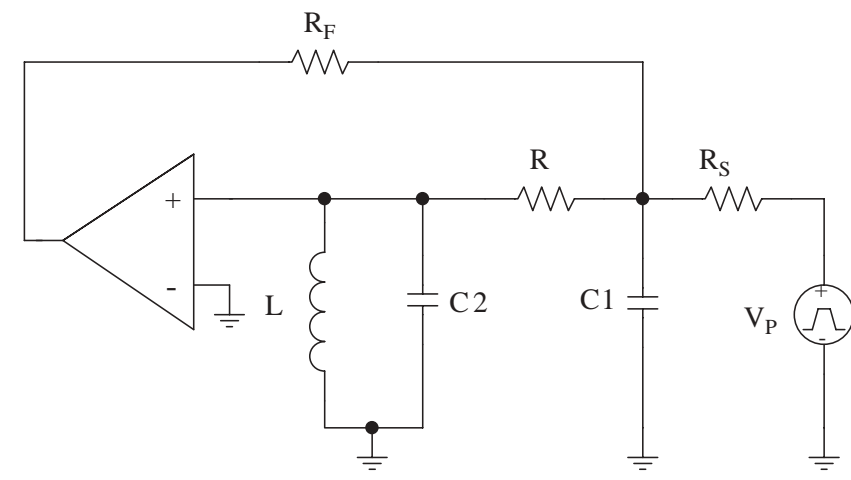

(b) Circuit structure with excitation across $C_{1}$

Fig. 81. Pulse-driven nonautonomous chaotic oscillator [Elwakil, 2002].

pulse-driven chaotic oscillator with fixed equilibrium points in the phase space.

As seen from Fig. 81(a) [Elwakil, 2002], the circuit equation is described by

$$
\left\{\begin{aligned}
L \dot{I}_{L}= & V_{C 2} \\
C_{2} \dot{V}_{C 2}= & \frac{V_{C 1}}{R}-\left(\frac{1}{R}+\frac{1}{R_{S}}+\frac{1}{R_{F}}\right) V_{C 2} \\
& -I_{L}+\frac{V_{P}}{R_{S}}+\frac{V_{N}}{R_{F}} \\
C_{1} \dot{V}_{C 1}= & \frac{V_{C 2}-V_{C 1}}{R}
\end{aligned}\right.
$$

where

$$
V_{N}=V_{C C} \operatorname{sgn}\left(V_{C 1}\right)= \begin{cases}V_{C C} & V_{C 1} \geq 0 \\ -V_{C C} & V_{C 1}<0\end{cases}
$$

and $V_{P}=V_{C C} \operatorname{sgn}\left(\operatorname{sgn}\left(\omega_{P} t\right)\right)$. Here, $\omega_{P}$ is the frequency of oscillation of the pulse-train.

Let $X=V_{C 1} / V_{C C}, Y=V_{C 2} / V_{C C}, Z=$ $R I_{L} / V_{C C}, \tau=\omega_{P} t, \epsilon_{c}=C_{1} / C_{2}, \alpha_{1}=L \omega_{P} / R$, $\alpha_{2}=R C_{2} \omega_{P}, \beta_{F}=R / R_{F}, \beta_{S}=R / R_{S}$. Then, system (83) becomes

$$
\begin{aligned}
\left(\begin{array}{c}
\epsilon_{c} \dot{X} \\
\dot{Y} \\
\dot{Z}
\end{array}\right)= & \left(\begin{array}{ccc}
-\frac{1}{\alpha_{2}} & \frac{1}{\alpha_{2}} & 0 \\
\frac{1}{\alpha_{2}} & -\frac{1+\beta_{F}+\beta_{S}}{\alpha_{2}} & -\frac{1}{\alpha_{2}} \\
0 & \frac{1}{\alpha_{1}} & 0
\end{array}\right)\left(\begin{array}{c}
X \\
Y \\
Z
\end{array}\right) \\
& +\left(\begin{array}{c}
0 \\
\frac{a}{\alpha_{2}} \\
0
\end{array}\right),
\end{aligned}
$$

where $\alpha_{1}, \alpha_{2}, \beta_{F}, \beta_{S}$ are real constants and the only parameter $a$ is defined by

$$
a= \begin{cases}\beta_{F}+\beta_{S} P(\tau) & X \geq 0 \\ -\beta_{F}+\beta_{S} P(\tau) & X<0\end{cases}
$$

in which

$$
P(\tau)=\operatorname{sgn}(\sin \tau)= \begin{cases}1 & \sin \tau \geq 0 \\ -1 & \sin \tau<0\end{cases}
$$

Figure 82(a) [Elwakil, 2002] displays the numerical simulation result of a 3 -scroll chaotic attractor, where $\alpha_{1}=0.05, \alpha_{2}=50, \beta_{F}=\beta_{S}=3, \tau=0.1 t$ and $\epsilon_{c}=0.2$. Clearly, the three equilibrium points are the origin and $(0,0, \pm 6)$.

Elwakil [2002] constructed a circuit diagram, as shown in Fig. 81(a), to verify the chaotic behaviors, where $L=1 \mathrm{mH}, C_{1}=10 \mathrm{nF}, C_{2}=100 \mathrm{nF}$, $R=5 \mathrm{k} \Omega, F_{F}=1 \mathrm{k} \Omega$. Moreover, $R_{S}$ is a variable $5 \mathrm{k} \Omega$ resistor; the resonant frequency of the $L C_{2}$ tank was approximately $16 \mathrm{kHz}$; the frequency of the driving pulse generator was in the range of $1-$ $20 \mathrm{kHz}$; the TL082 operational amplifier was biased from $\pm 5 \mathrm{~V}$ supplies and the pulse generator output was also fixed to $\pm 5 \mathrm{~V}$. Figure 82 (b) [Elwakil, 2002] displays the observed chaotic attractor in the $V_{C 1}-V_{C 2}(X-Y)$ plane, where $R_{S}=1.636 \mathrm{k} \Omega$. Note that it is particularly difficult to measure the current in the inductor $I_{L}(Z)$.

Recall that the passive structure of Chua's circuit is a two-node structure. In Fig. 81(a) [Elwakil, 2002], it is chosen to excite one of these nodes, namely, the one across $C_{2}$. In the following, the other node, namely, the one across $C_{1}$, is alternatively excited, as shown in Fig. 81(b) 


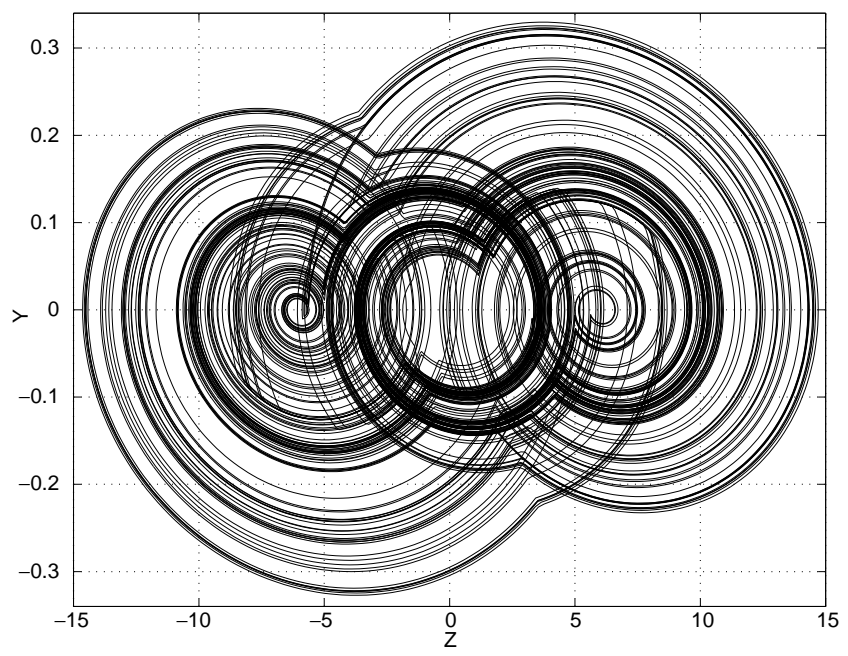

(a) 3-Scroll attractor generated via numerical simulation for $\left(\beta_{F}, \beta_{S}\right)=(2,3)$

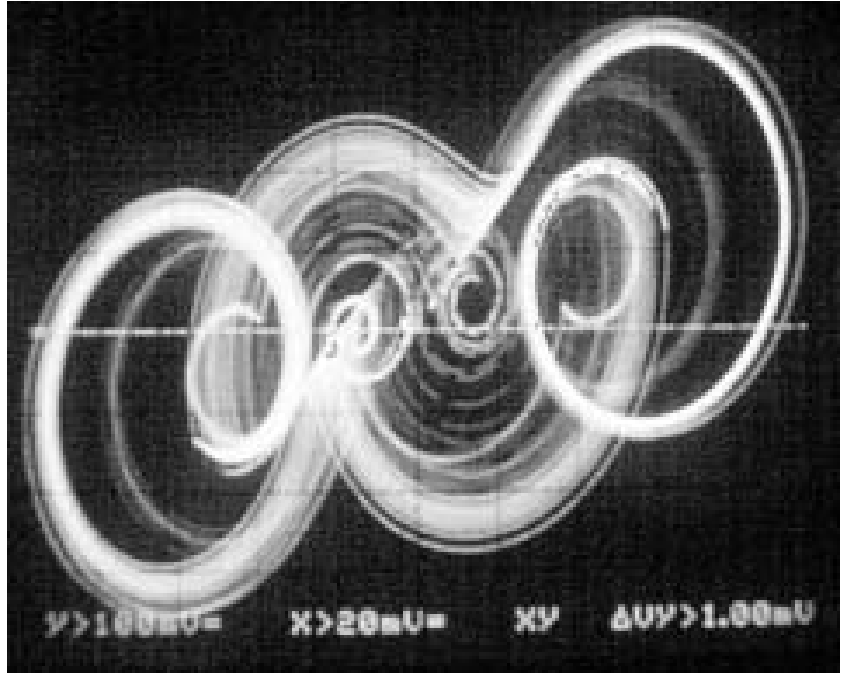

(b) Experimental observation of 3-Scroll attractor in the $V_{C 1}-V_{C 2}$ plane $(X$-axis: $20 \mathrm{mV} / \operatorname{div}, Y$-axis: $100 \mathrm{mV} /$ div)

Fig. 82. Pulse-driven chaotic attractor [Elwakil, 2002].

[Elwakil, 2002]. The corresponding circuit equation is described by

$$
\begin{aligned}
\left(\begin{array}{c}
\epsilon_{c} \dot{X} \\
\dot{Y} \\
\dot{Z}
\end{array}\right)= & \left(\begin{array}{ccc}
1 & -\left(1+\beta_{F}+\beta_{S}\right) & 0 \\
\frac{1}{\alpha_{2}} & -\frac{1}{\alpha_{2}} & -\frac{1}{\alpha_{2}} \\
0 & \frac{1}{\alpha_{1}} & 0
\end{array}\right)\left(\begin{array}{l}
X \\
Y \\
Z
\end{array}\right) \\
& +\left(\begin{array}{l}
a \\
0 \\
0
\end{array}\right),
\end{aligned}
$$

where $a$ is defined in (85) with the switching condition dependent on $y$ instead of $x$.

Figure 83 [Elwakil, 2002] displays the observed 4 -scroll chaotic attractor, where $\epsilon_{c}=0.1, \alpha_{1}=0.07$, $\alpha_{2}=0.5$ and $\beta_{F}=\beta_{S}=3$. A single positive Lyapunov exponent equal to 0.0089 is also calculated from the $y$ time series.

\subsection{Multiscroll chaotic attractors in the fractional order systems}

In this subsection, one simple fractional order system is constructed for generating $n$-scroll chaotic attractors [Ahmad, 2005].
Recently, Ahmad [2005] introduced a simple fractional chaotic oscillator described by

$$
\left\{\begin{array}{l}
\frac{d x_{1}^{\alpha}}{d t^{\alpha}}=x_{2} \\
\frac{d x_{2}}{d t}=x_{3} \\
\frac{d x_{3}}{d t}=-a\left(x_{1}+x_{2}+x_{3}-f\left(x_{1}\right)\right) \\
y=x_{1},
\end{array}\right.
$$

where $d x_{1}^{\alpha} / d t^{\alpha}$ is the fractional derivative of $x_{1}$ with order $\alpha, a$ is the control parameter for this oscillator, $f\left(x_{1}\right)=\operatorname{sgn}\left(x_{1}\right)$ is the nonlinearity, and $y$ is the system output.

Clearly, the order of system (87) is $2+\alpha$. For integer order case, system (87) can generate doublescroll chaotic attractors for $0.49<a<1$; for fractional order case, the range of parameter $a$ for creating chaos depends on the given fractional order. To create multiscroll chaotic attractors from fractional system (87), Ahmad slightly modified the nonlinearity $f\left(x_{1}\right)$ as follows:

$f\left(x_{1}\right)=\operatorname{sgn}\left(x_{1}\right)+\sum_{k=1}^{N}\left(\operatorname{sgn}\left(x_{1}-k\right)+\operatorname{sgn}\left(x_{1}+k\right)\right)$. 


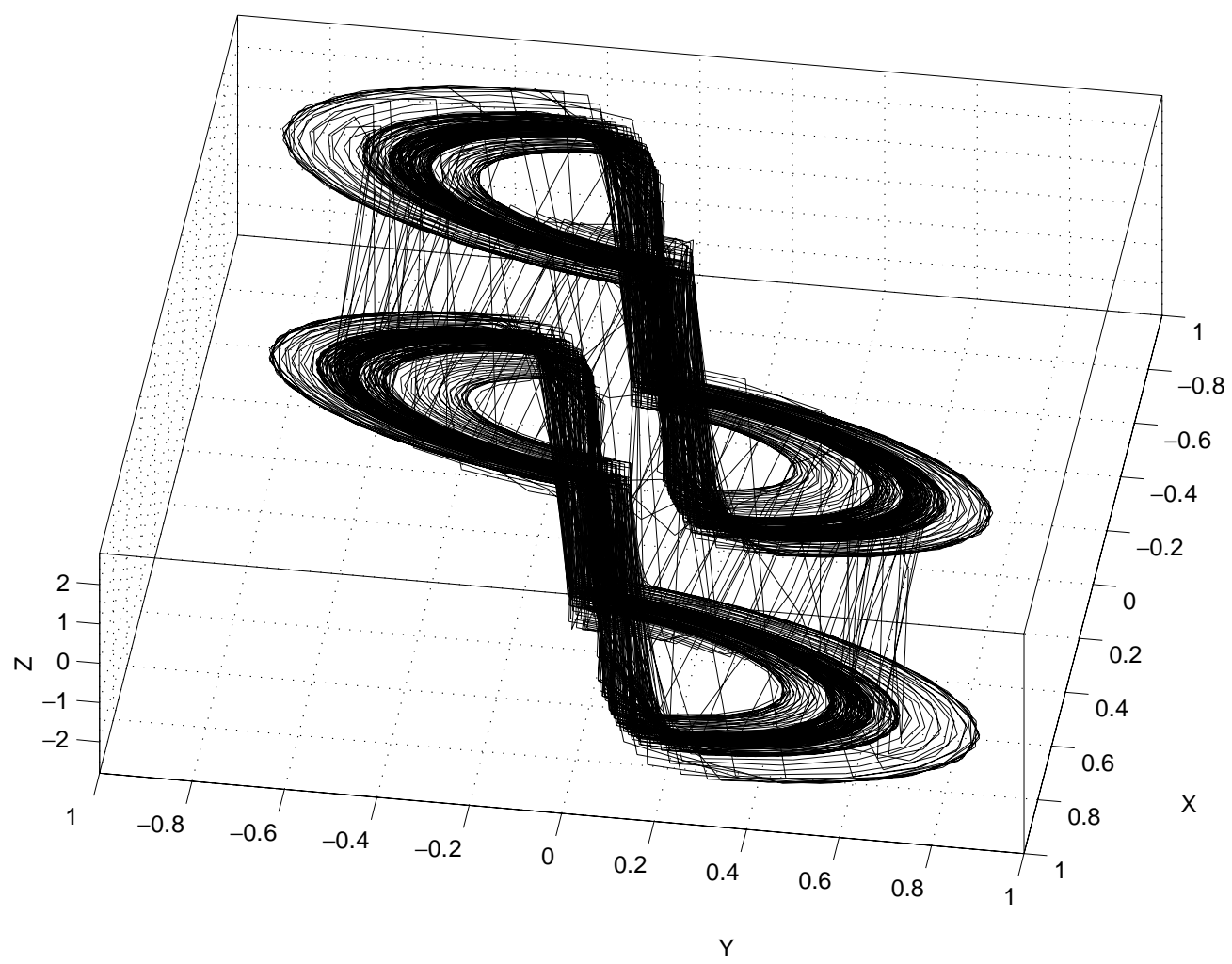

Fig. 83. 4-Scroll chaotic attractor observed from the model of alternatively excited circuit [Elwakil, 2002].

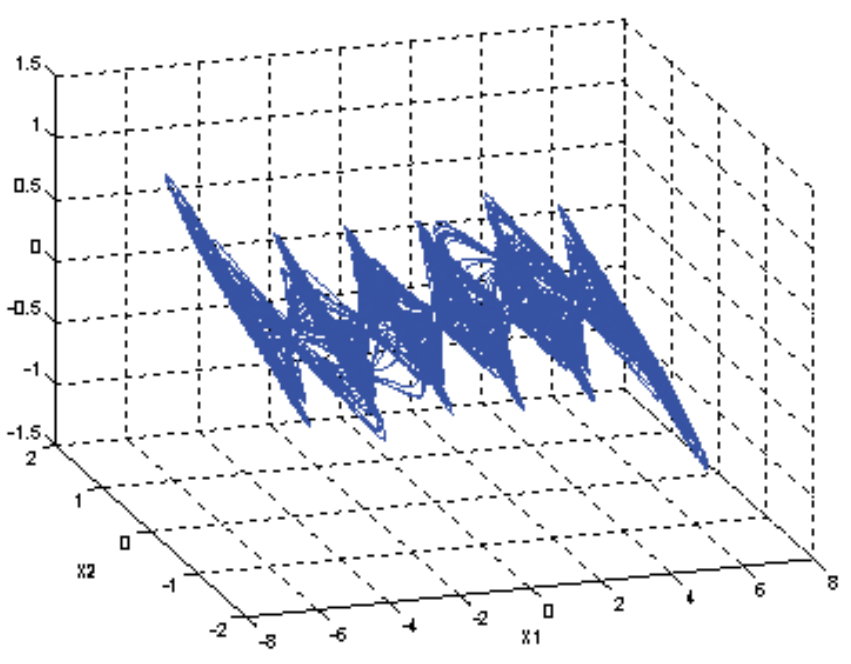

Fig. 84. 6-Scroll chaotic attractor observed from integer order system (87) with $\alpha=1, a=0.4$ [Ahmad, 2005].

When $\alpha=1, a=0.4$, system (87) with (88) has integer order and a 6-scroll chaotic attractor as shown in Fig. 84 [Ahmad, 2005]. When $\alpha=0.5, a=$ 0.01 , system (87) with (88) has fractional order 2.5 and a 6 -scroll chaotic attractor as shown in Fig. 85 [Ahmad, 2005].

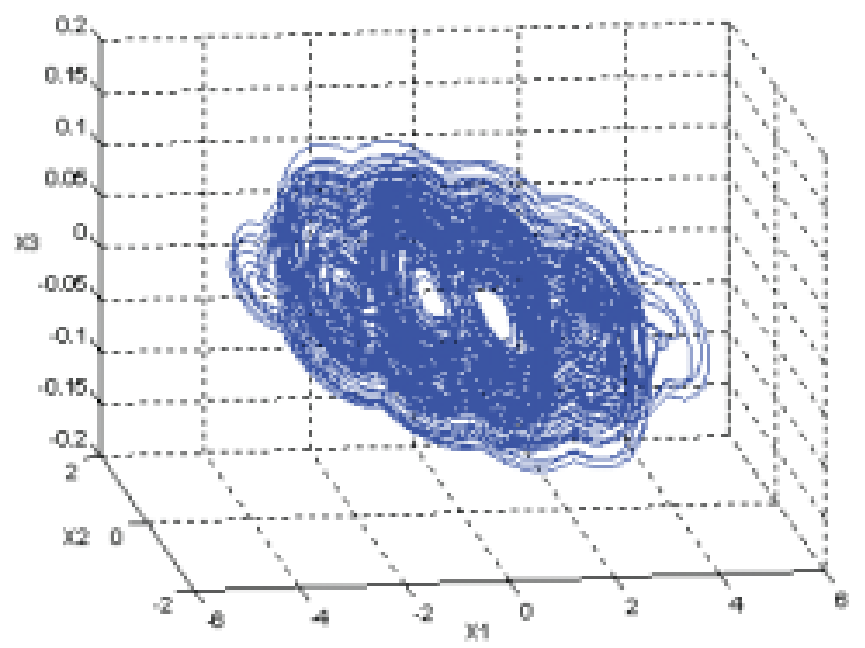

Fig. 85. 6-Scroll chaotic attractor observed from fractional order system (87) with $\alpha=0.5, a=0.01$ [Ahmad, 2005].

\section{Implementation and Applications of Multiscroll Chaotic Attractors}

This section briefly summarizes digital implementation, control, synchronization and some potential real-world applications of multiscroll chaotic attractors. 


\subsection{Generating multiscroll chaotic attractors via digital circuit implementation}

Eguchi et al. [1999] proposed a novel digital circuit for generating multiscroll chaotic attractors.

Let $x=v_{C_{1}}, y=v_{C_{2}}, z=i_{L}, A^{\prime}=1 / R$, $B^{\prime}=C_{1}, C^{\prime}=C_{2}, D^{\prime}=L$. Then, Chua's circuit (1) becomes

$$
\left\{\begin{array}{l}
B^{\prime} \frac{d x}{d t}=A^{\prime}(y-x)-g(x) \\
C^{\prime} \frac{d y}{d t}=A^{\prime}(x-y)+z \\
D^{\prime} \frac{d z}{d t}=-y,
\end{array}\right.
$$

with $g(x)=m_{1} x+1 / 2\left(m_{0}-m_{1}\right)\left(\left|x+b_{1}\right|-\left|x-b_{1}\right|\right)$.

Consider the following difference trans- formation:

$$
\begin{gathered}
\frac{d x}{d t}=\frac{X_{n+1}-X_{n}}{2^{E}}, \quad \frac{d y}{d t}=\frac{Y_{n+1}-Y_{n}}{2^{E}}, \\
\frac{d z}{d t}=\frac{Z_{n+1}-Z_{n}}{2^{E}}
\end{gathered}
$$

where $E$ is an integer parameter. The corresponding difference equation of the general Chua's circuit with multiple breakpoints is described by

$$
\left\{\begin{array}{l}
X_{n+1}=B \frac{A \frac{\left(Y_{n}-X_{n}\right)}{2^{m}}-G\left(X_{n}\right)}{2^{m-E}}+X_{n} \\
Y_{n+1}=C \frac{Z_{n}-A \frac{Y_{n}-X_{n}}{2^{m}}}{2^{m-E}}+Y_{n} \\
Z_{n+1}=-D \frac{Y_{n}}{2^{m-E}}+Z_{n},
\end{array}\right.
$$

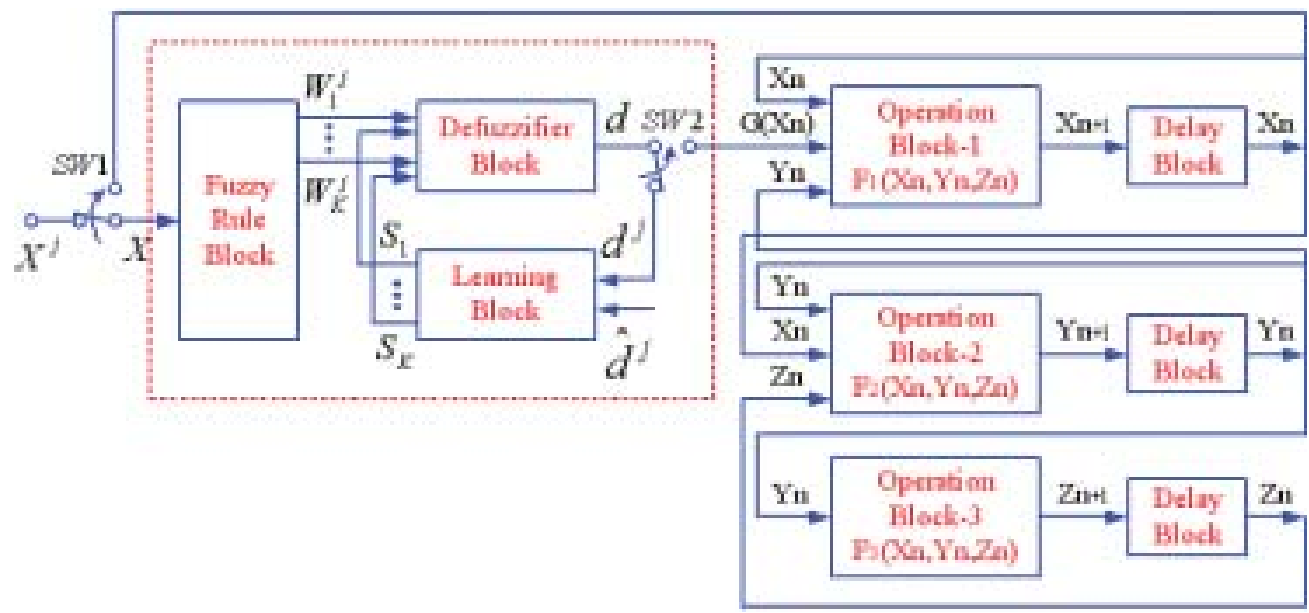

Fig. 86. General circuit architecture of the digital chaotic circuit.

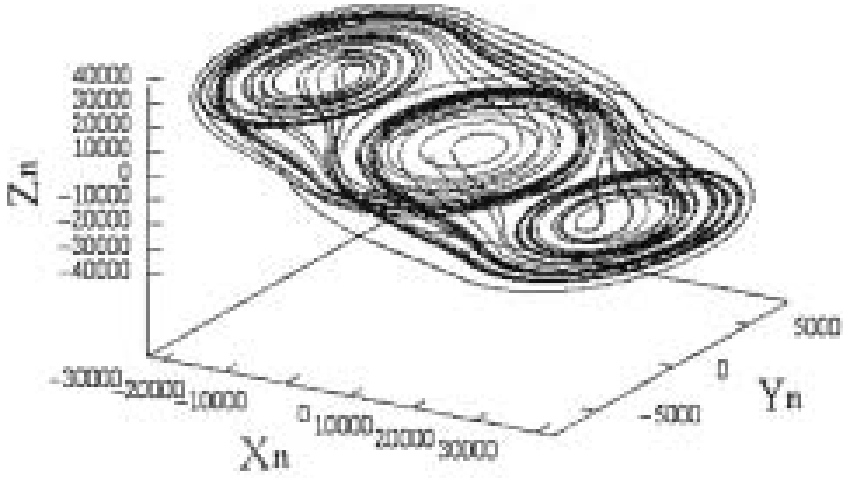

(a) 3-Scroll chaotic attractor

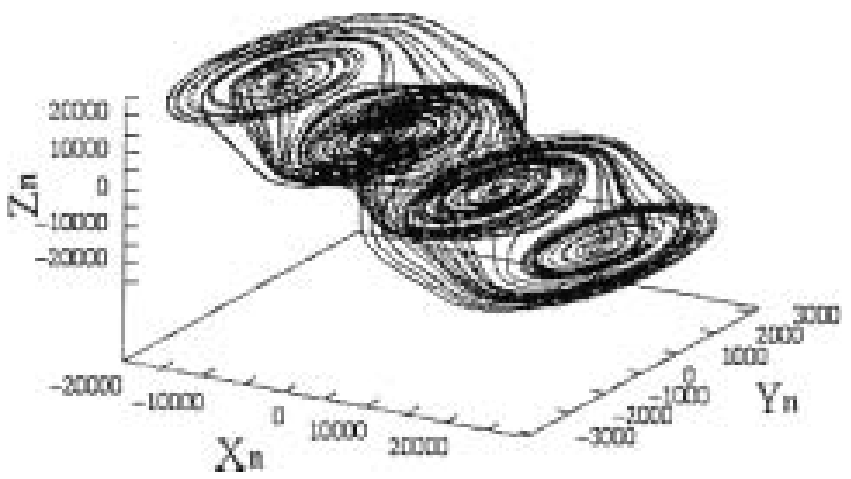

(b) 4-Scroll chaotic attractor

Fig. 87. Numerical simulation of the digital circuit [Eguchi et al., 1999]. 
where $A=\left[A^{\prime} 2^{m}\right], B=\left[1 / B^{\prime}\right], C=\left[1 / C^{\prime}\right]$, $D=\left[1 / D^{\prime}\right], m$ are integer parameters on the interval $\left[0,2^{m+1}\right]$ and $G\left(X_{n}\right)$ is a PWL function with $k$ breakpoints, in which [.] is the Gauss integer function. For simplicity, denote $X_{n+1}=$ $F_{1}\left(X_{n}, Y_{n}, Z_{n}\right), Y_{n+1}=F_{2}\left(X_{n}, Y_{n}, Z_{n}\right)$, and $Z_{n+1}=$ $F_{3}\left(X_{n}, Y_{n}, Z_{n}\right)$. Finally, the complicated nonlinear function $G\left(X_{n}\right)$ is determined electronically by using supervised learning based on a neuro-fuzzy scheme.

Figure 86 displays the general circuit architecture of the digital chaos circuit (89). It includes a nonlinear function block, three operation blocks and three delay blocks.

Eguchi et al. [1999] numerically confirmed the validity of the circuit algorithm. In all numerical simulations, the membership functions are defined as

$$
\begin{aligned}
& \text { MS1 }: \mu_{A 1}(x)=2^{m+1} \ominus 10 x, \\
& \text { MS2 }: \mu_{A 2}(x)= \begin{cases}10 x & \text { if } x<\frac{2^{m}}{5} \\
3 \times 2^{m}-5 x & \text { if } x \geq \frac{2^{m}}{5}\end{cases}
\end{aligned}
$$

MS3 $: \mu_{A 3}(x)= \begin{cases}5 x \ominus 2^{m} & \text { if } x<3 \times \frac{2 m}{5} \\ \left(5 \times 2^{m}\right) \ominus 5 x & \text { if } x \geq 3 \times \frac{2^{m}}{5}\end{cases}$

MS4 $: \mu_{A 4}(x)= \begin{cases}5 x \ominus\left(3 \times 2^{m}\right) & \text { if } x<2^{m} \\ 2^{m+2} \ominus 2 x & \text { if } x \geq 2^{m}\end{cases}$

MS5 : $\mu_{A 5}(x)=2 x \ominus 2^{m+1}$,

where $x$ is the input variable and $\ominus$ is the bounded difference operator. Figure 87 [Eguchi et al., 1999] shows the simulated 3,4-scroll chaotic attractors obtained from the digital circuit.

Eguchi et al. [1999] also constructed an FPGA chaotic circuit for creating $n$-scroll chaotic attractors $(n=1, \ldots, 4)$ based on a CAD tool, VerilogHDL. Figure 88 [Eguchi et al., 1999] shows the FPGA functional block allocation result for the synthesized circuit, where the number of inference rules $k$ was set to 5 , the parameter $m$ was set to 14 , and the SRAM is excluded from the chip as an external memory.

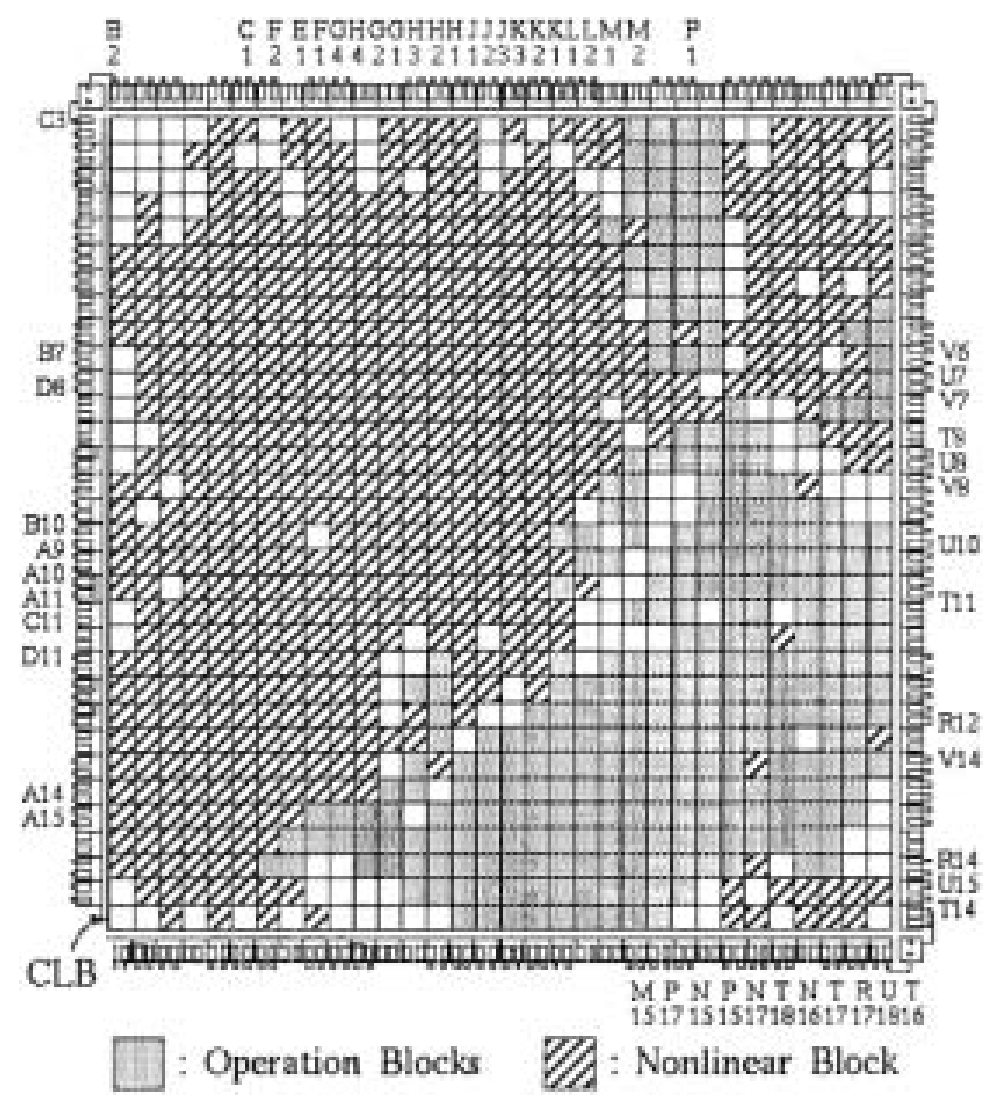

Fig. 88. The functional block allocation view of the FPGA implementing the digital circuit [Eguchi et al., 1999]. 


\subsection{Digitized $n$-scroll chaotic attractors model for secure communication}

Tang et al. [2001a] proposed a secure digital communication system using digitized $n$-scroll chaotic attractors.

Consider Chua's circuit (8) with $h\left(x_{1}\right)=a x_{1}+$ $b x_{1}\left|x_{1}\right|$, where $\left(x_{1}, x_{2}, x_{3}\right)^{T}$ is the state vector. Using a bilinear transformation, the discrete-time equation of (8) is obtained as

$$
\begin{aligned}
\left(\begin{array}{l}
x_{1}(k+1) \\
x_{2}(k+1) \\
x_{3}(k+1)
\end{array}\right)= & \left(\begin{array}{lll}
\phi_{11} & \phi_{12} & \phi_{13} \\
\phi_{21} & \phi_{22} & \phi_{23} \\
\phi_{31} & \phi_{32} & \phi_{33}
\end{array}\right)\left(\begin{array}{l}
x_{1}(k) \\
x_{2}(k) \\
x_{3}(k)
\end{array}\right) \\
& +\left(\begin{array}{l}
\gamma_{1} \\
\gamma_{2} \\
\gamma_{3}
\end{array}\right) h\left(x_{1}(k)\right)
\end{aligned}
$$

where

$$
\begin{gathered}
\phi_{11}=\frac{4 \alpha T^{2}+\zeta_{1}}{\zeta_{1}}, \quad \phi_{12}=\frac{8 \alpha T}{\zeta_{1}}, \quad \phi_{13}=\frac{4 \alpha T^{2}}{\zeta_{1}} \\
\phi_{21}=\frac{8 \alpha T^{3}+4 T \zeta_{1}}{\zeta_{1} \zeta_{2}}, \quad \phi_{22}=\frac{16 \alpha T^{2}+\left(8-\zeta_{2}\right) \zeta_{1}}{\zeta_{1} \zeta_{2}} \\
\phi_{23}=\frac{8 \alpha T^{3}+4 T \zeta_{1}}{\zeta_{1} \zeta_{2}}, \quad \phi_{31}=\frac{4 \alpha \beta T^{4}+2 \beta T^{2} \zeta_{1}}{\zeta_{1} \zeta_{2}} \\
\phi_{32}=\frac{8 \alpha \beta T^{3}+4 \beta T \zeta_{1}}{\zeta_{1} \zeta_{2}}, \\
\phi_{33}=\frac{4 \alpha \beta T^{4}+\left(\zeta_{2}-2 \beta T^{2}\right) \zeta_{1}}{\zeta_{1} \zeta_{2}}, \quad \gamma_{1}=\frac{-2 \alpha T \zeta_{2}}{\zeta_{1}}, \\
\gamma_{2}=\frac{-4 \alpha T^{2}}{\zeta_{1}}, \quad \gamma_{3}=\frac{-2 \alpha \beta T^{3}}{\zeta_{1}}, \\
\zeta_{1}=8+4 T(1+\alpha a)+2 T^{2}(\beta+\alpha+\alpha a)+\alpha \beta a T^{3}, \\
\zeta_{2}=4+2 T+\beta T^{2}, \\
h\left(x_{1}(k)\right)=a x_{1}(k)+b x_{1}(k)\left|x_{1}(k)\right|
\end{gathered}
$$

in which the sampling time $T>0$, and $\alpha, \beta, a, b$ are constant parameters. Denote $(90)$ as $\mathbf{x}(k+1)=$ $\Phi \mathbf{x}(k)+\Gamma h\left(x_{1}(k)\right)$.

To generate $n$-scroll chaotic attractors, Tang et al. [2001a] extended the nonlinear function as follows:

$$
h(u(k))=a u(k)+b u(k)|u(k)|,
$$

where, if $x(k)$ in $[-(2 m-1)|a| / b,(2 m-1)|a| / b]$ for $m=0, \pm 1, \ldots, \pm(n / 2-1)$, then $u(k)=$ $x(k)+2 m a / b$; otherwise, $u(k)=x(k)+(n-2) a / b$.

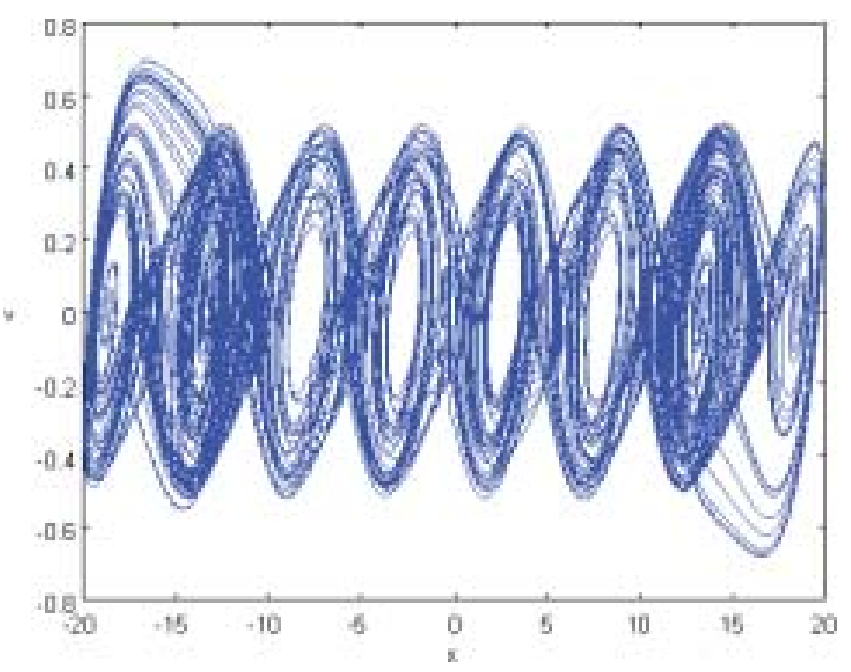

Fig. 89. Digital 8-scroll attractor from modified Chua's circuit.

Figure 89 displays a digital 8-scroll chaotic attractor.

Next, consider the following two discrete-time autonomous chaotic systems:

$$
\begin{aligned}
S_{1}: \mathbf{w}(k+1) & =\Phi_{1} \mathbf{w}(k)+\Gamma_{1} h_{1}(\mathbf{w}(k)) \\
S_{2}: \mathbf{v}(k+1) & =\Phi_{2} \mathbf{v}(k)+\Gamma_{2} h_{2}(\mathbf{v}(k)),
\end{aligned}
$$

where $\mathbf{w}(k), \mathbf{v}(k) \in \mathbf{R}^{3}$ are state vectors. Moreover, if $\exists \mathbf{M} \in \mathbf{R}^{3 \times 3}$, such that $\lim _{k \rightarrow \infty} \| \mathbf{w}(k)-$ $\mathbf{M v}(k) \|=0$, then subsystems $S_{1}, S_{2}$ are called M-synchronization.

Tang et al. [2001a] designed a secure digital communication scheme using chaotic phase shift keying (CPSK) method. Figure 90 shows the transmitter $T_{r}$ and the receiver $R_{e}$. The transmitter observer $T_{r}$ is described by

$$
\mathbf{w}(k+1)=\mathbf{M}_{i}^{-1} \Phi \mathbf{M w}(k)+\mathbf{M}_{i}^{-1} \Gamma u(k),
$$

where $\mathbf{w}(k) \in \mathbf{R}^{3}$ is the state vector of $T_{r}$ at time $k, \Phi \in \mathbf{R}^{3 \times 3}$ is a stable matrix, $\Gamma \in \mathbf{R}^{3 \times 1}, \mathbf{M}_{i} \in$ $\mathbf{R}^{3 \times 3}$ is a nonsingular synchronization matrix, and $u(k) \in \mathbf{R}$ is a nonlinear feedback function.

The receiver $R_{c}$ is given by

$$
\begin{aligned}
\mathbf{v}(k+1)= & \left(\Phi+\mathbf{H}_{i} \eta_{i} \mathbf{M}_{i}^{-1}\right) \mathbf{v}(k)+\Gamma \mathbf{u}(k) \\
& -\mathbf{H}_{i} s(k),
\end{aligned}
$$

where $\mathbf{H}_{i} \in \mathbf{R}^{3 \times 1}$.

For each $i$, define the error function as follows:

$$
\varepsilon_{i}^{2}=\sum_{k=\tau_{m}}^{\tau}\left(\mathbf{s}(k)-\eta_{i} \mathbf{M}_{i}^{-1} \mathbf{v}(k)\right)^{2},
$$

where $\tau$ is the signal interval and $\tau_{m}$ is the minimum number of steps to realize synchronization. 


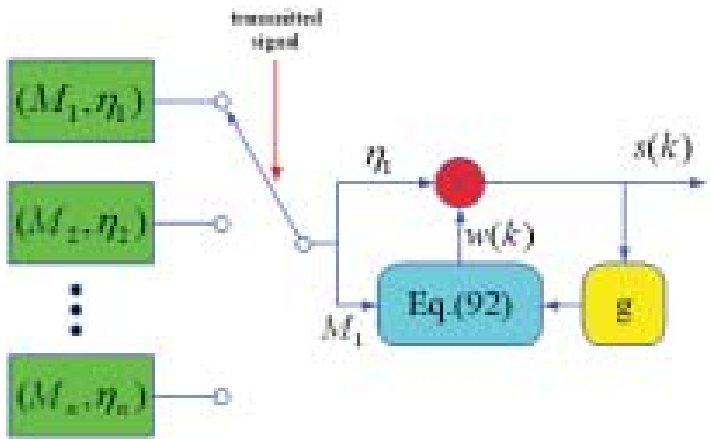

(a) Transmitter $T_{r}$

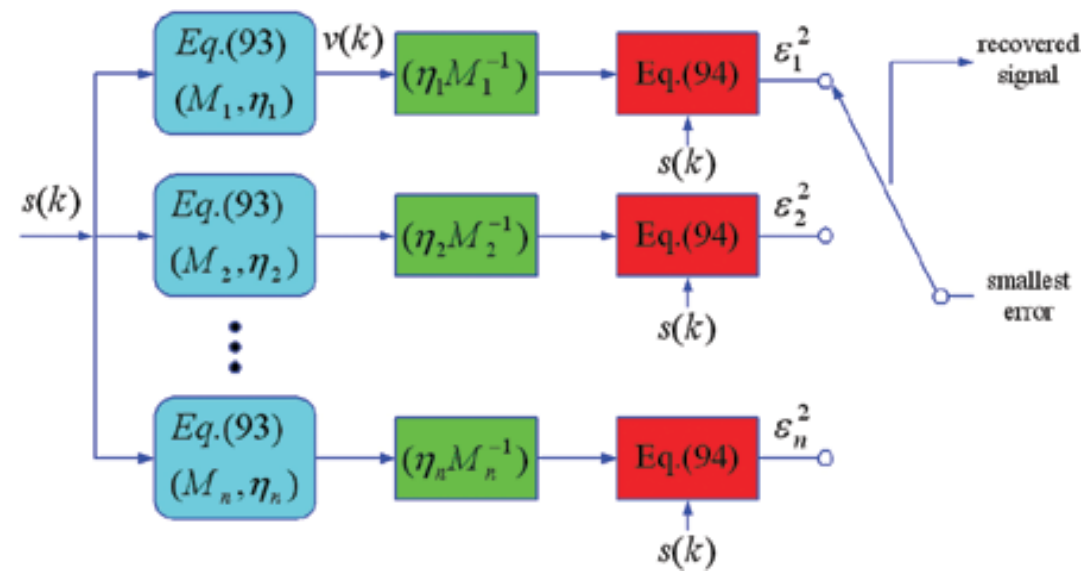

(b) Receiver $R_{e}$

Fig. 90. Transmitter and receiver in M-CPSK.

Let $\eta_{1}=\eta_{2}=\left[\begin{array}{lll}1 & 0 & 0\end{array}\right]$ and

$$
\mathbf{M}_{1}=\left(\begin{array}{lll}
2 & 0 & 0 \\
0 & 4 & 0 \\
0 & 0 & 2
\end{array}\right), \quad \mathbf{M}_{2}=\left(\begin{array}{rrr}
-2 & 0 & 0 \\
0 & 3 & 0 \\
0 & 0 & -2
\end{array}\right) \text {. }
$$

At the receiver end, $\mathbf{H}_{i} \in \mathbf{R}^{n \times 1}$ is designed such that the desired eigenvalues are $(0,0.001,-0.001)$ and $(0,0.002,-0.002)$ for $\mathbf{H}_{1}$ and $\mathbf{H}_{2}$, respectively. Figure 91(a) displays the error between $\eta_{i} \times$ $\mathbf{M}_{i}^{-1} \mathbf{v}$ and $\mathbf{s}$. Figure $91(\mathrm{~b})$ shows the relationship of the probability bit error rate (BER) with the signal-to-noise ratio (SNR).

\subsection{Unidirectionally coupled synchronization of multiscroll chaotic attractors}

$\mathrm{Yu}$ et al. [2004b] produced a unidirectionally coupled synchronization scheme for $n$-scroll chaotic attractors from the 4D modified Chua's circuit [Chen \& Lü, 2002; Lü et al., 2002g; Makoto et al., 1999].
The 6 -scroll driving system is described by

$$
\left\{\begin{array}{l}
\dot{x}_{1}=\alpha\left(y_{1}-x_{1}-g\left(x_{1}\right)\right) \\
\dot{y}_{1}=x_{1}-y_{1}+z_{1} \\
\dot{z}_{1}=-\beta\left(y_{1}-w_{1}\right) \\
\dot{w}_{1}=-\gamma_{2}\left(z_{1}+\gamma_{1} w_{1}\right)
\end{array}\right.
$$

where

$$
\begin{aligned}
g\left(x_{1}\right)= & m_{5} x_{1}+\frac{1}{2} \sum_{i=1}^{5}\left(m_{i-1}-m_{i}\right) \\
& \times\left(\left|x_{1}+b_{i}\right|-\left|x_{1}-b_{i}\right|\right) .
\end{aligned}
$$

And the corresponding response system is given by

$$
\left\{\begin{array}{l}
\dot{x}_{2}=\alpha\left(y_{2}-x_{2}-g\left(x_{2}\right)\right)+\delta_{x}\left(x_{1}-x_{2}\right) \\
\dot{y}_{2}=x_{2}-y_{2}+z_{2}+\delta_{y}\left(y_{1}-y_{2}\right) \\
\dot{z}_{2}=-\beta\left(y_{2}-w_{2}\right) \\
\dot{w}_{2}=-\gamma_{2}\left(z_{2}+\gamma_{1} w_{2}\right),
\end{array}\right.
$$

where

$$
\begin{aligned}
g\left(x_{2}\right)= & m_{5} x_{2}+\frac{1}{2} \sum_{i=1}^{5}\left(m_{i-1}-m_{i}\right) \\
& \times\left(\left|x_{2}+b_{i}\right|-\left|x_{2}-b_{i}\right|\right) .
\end{aligned}
$$




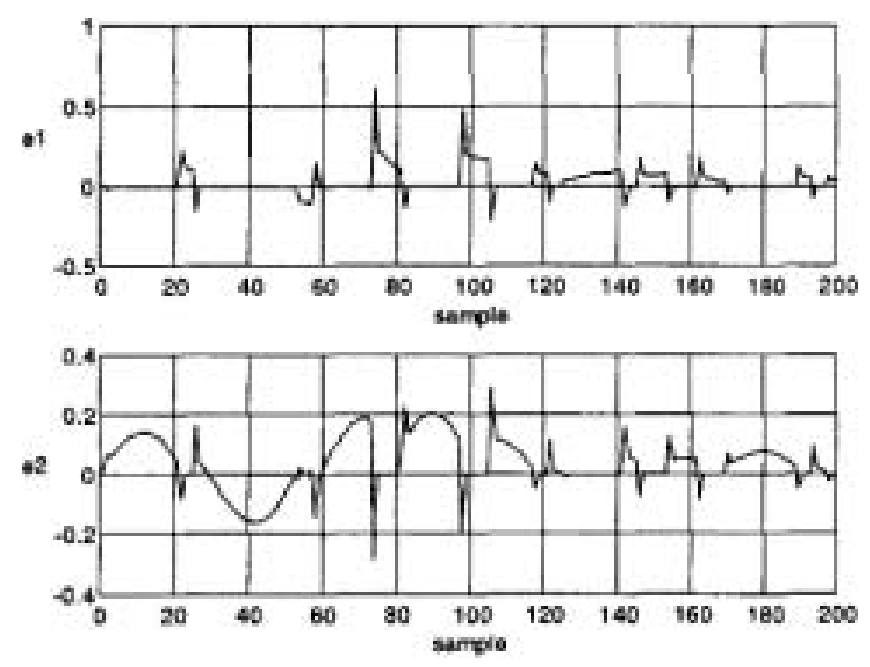

(a) Error between $\eta_{i} \mathbf{M}_{i}^{-1} \mathbf{v}$ and $\mathbf{s}$

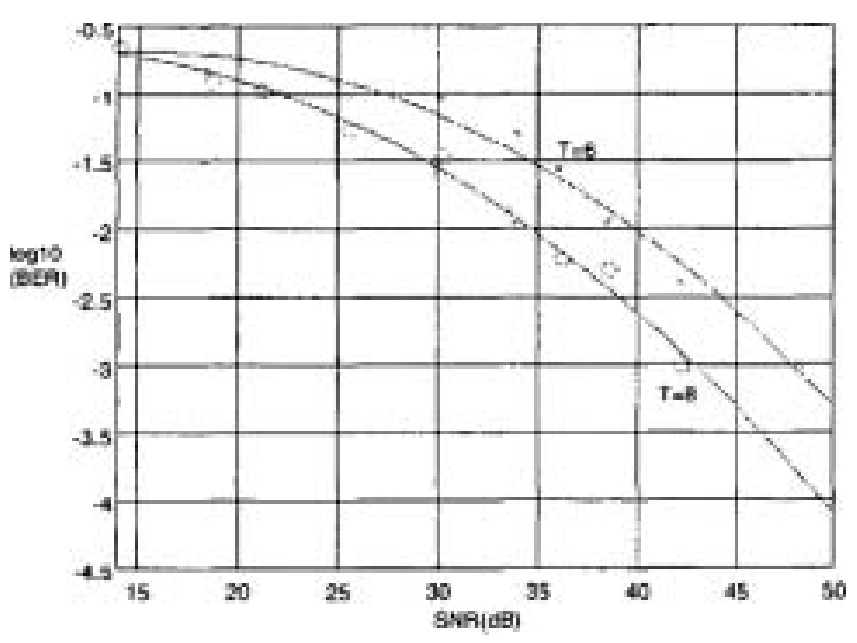

(b) BER under AWGH noisy transmission channel

Fig. 91. Numerical simulations of the M-CPSK scheme.

Here, assume that $\alpha=9.934, \beta=14.334$, $\gamma_{1}=27.333, \gamma_{2}=0.0497, m_{0}=m_{2}=m_{4}=$ $m_{a}=-1.246, m_{1}=m_{3}=m_{5}=m_{b}=-0.6724$, and $b_{1}=10, b_{2}=29.2466, b_{3}=49.2466, b_{4}=$ $68.4931, b_{5}=88.4931$.

$\mathrm{Yu}$ et al. [2004b] derived the following conditions for chaos synchronization.

Lemma 1 [Yu et al., 2004b]. Suppose that there exist coupling coefficients $\delta_{x}>0$ and $\delta_{y} \geq 0$ such that $\alpha m_{a}+\delta_{x}+\left(\alpha+\alpha m_{a}+\delta_{x}\right) \delta_{y}>0$ in the unidirectionally coupled systems (59) and (60). Then, the response system (60) is globally asymptotically synchronizing with the drive system (59).

When $\delta_{x}=23, \delta_{y}=0, \alpha m_{a}+\delta_{x}+\left(\alpha+\alpha m_{a}+\right.$ $\left.\delta_{x}\right) \delta_{y}=10.6222>0$, from Lemma 1 , systems (59) and (60) can be synchronized; when $\delta_{x}=\delta_{y}=0$, systems (59) and (60) obviously cannot realize synchronization since there is no coupling between them.

Yu et al. [2004b] constructed a circuit diagram to experimentally verify the unidirectionally coupled synchronization. Figure 92 [Yu et al., 2004b] shows the circuitry for the two unidirectionally coupled identical 6-scroll systems, where $N_{R}$ is the function part within the dotted line shown in Fig. 60 [Yu et al., 2004b]. When the coupling resistor $R_{E}=0$, the coupling coefficients $\delta_{x}=$ $\left(C_{2} R / C_{1}\right) R_{E}=0$ and $\delta_{y}=0$, then the two 6 -scroll chaotic attractors cannot realize synchronization, as shown in Fig. 93(a) [Yu et al., 2004b]. When the coupling resistor $R_{E}=0.7 \mathrm{k} \Omega$, the coupling coefficients $\delta_{x}=\left(C_{2} R / C_{1}\right) R_{E}=23$ and $\delta_{y}=0$, the two 6-scroll chaotic attractors can reach synchronization, as shown in Fig. 93(b) [Yu et al., 2004b].

$\mathrm{Yu}$ et al. [2004b] also investigated a unidirectionally coupled synchronization scheme for $n$-scroll hyperchaotic attractors generated from the MCK circuit.

The 6-scroll hyperchaotic MCK drive system is described by

$$
\left\{\begin{array}{l}
\dot{x}_{1}=\alpha\left(g\left(y_{1}-x_{1}\right)-z_{1}\right) \\
\dot{y}_{1}=\beta\left(-g\left(y_{1}-x_{1}\right)-w_{1}\right) \\
\dot{z}_{1}=x_{1}+z_{1} \\
\dot{w}_{1}=\gamma y_{1}
\end{array}\right.
$$

where

$$
\begin{aligned}
g\left(y_{1}-x_{1}\right)= & m_{5}\left(y_{1}-x_{1}\right)+\frac{1}{2} \sum_{i=1}^{5}\left(m_{i-1}-m_{i}\right) \\
& \times\left(\left|y_{1}-x_{1}+c_{i}\right|-\left|y_{1}-x_{1}-c_{i}\right|\right) .
\end{aligned}
$$

The corresponding response system is given by

$$
\left\{\begin{array}{l}
\dot{x}_{2}=\alpha\left(g\left(y_{2}-x_{2}\right)-z_{2}\right)+\delta_{x}\left(x_{1}-x_{2}\right) \\
\dot{y}_{2}=\beta\left(-g\left(y_{2}-x_{2}\right)-w_{2}\right)+\delta_{y}\left(y_{1}-y_{2}\right) \\
\dot{z}_{2}=x_{2}+z_{2}+\delta_{z}\left(z_{1}-z_{2}\right) \\
\dot{w}_{2}=\gamma y_{2}+\delta_{w}\left(w_{1}-w_{2}\right)
\end{array}\right.
$$

where $\delta_{x}, \delta_{y}, \delta_{z}, \delta_{w}$ are the coupling coefficients, and

$$
\begin{aligned}
g\left(y_{2}-x_{2}\right)= & m_{5}\left(y_{2}-x_{2}\right)+\frac{1}{2} \sum_{i=1}^{5}\left(m_{i-1}-m_{i}\right) \\
& \times\left(\left|y_{2}-x_{2}+c_{i}\right|-\left|y_{2}-x_{2}-c_{i}\right|\right) .
\end{aligned}
$$




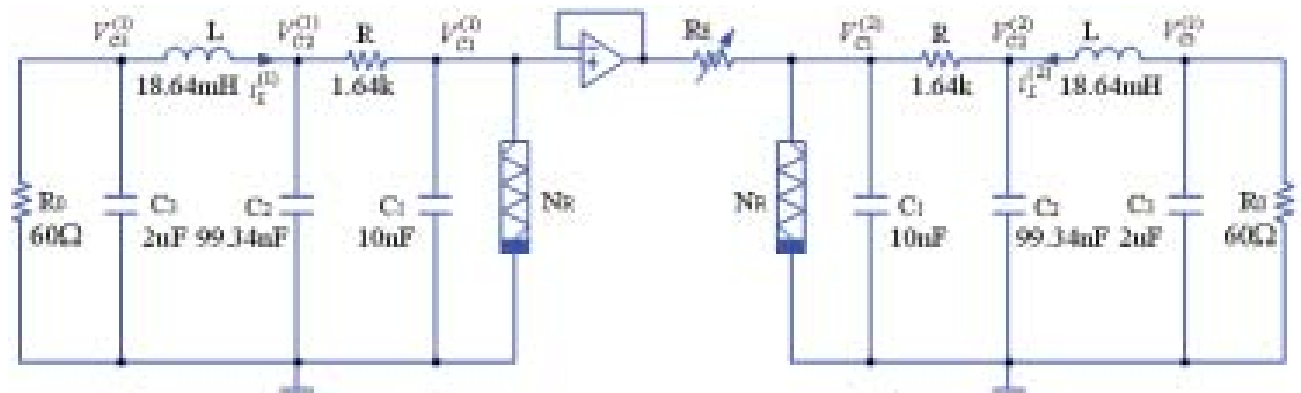

Fig. 92. Circuitry for unidirectionally coupled synchronization between two 6-scroll chaotic attractors [Yu et al., 2004b].

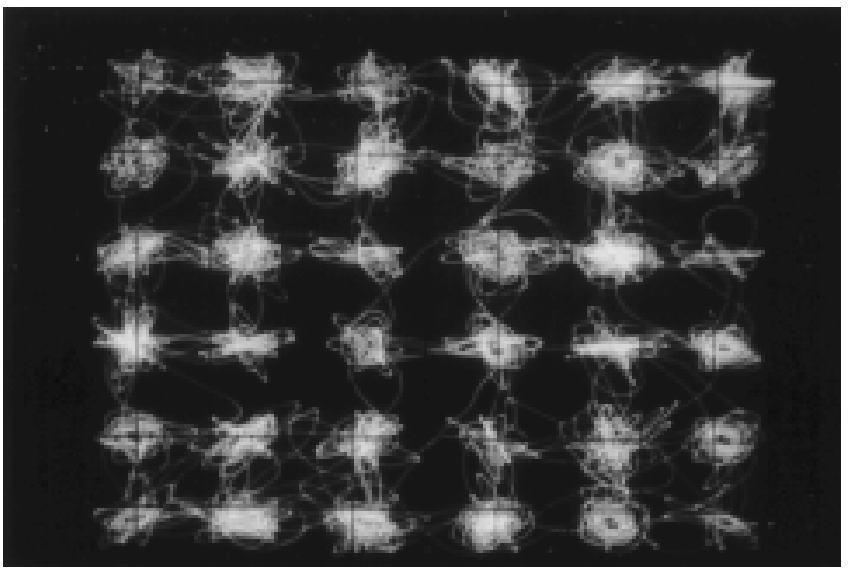

(a) Asynchronous when there is no coupling

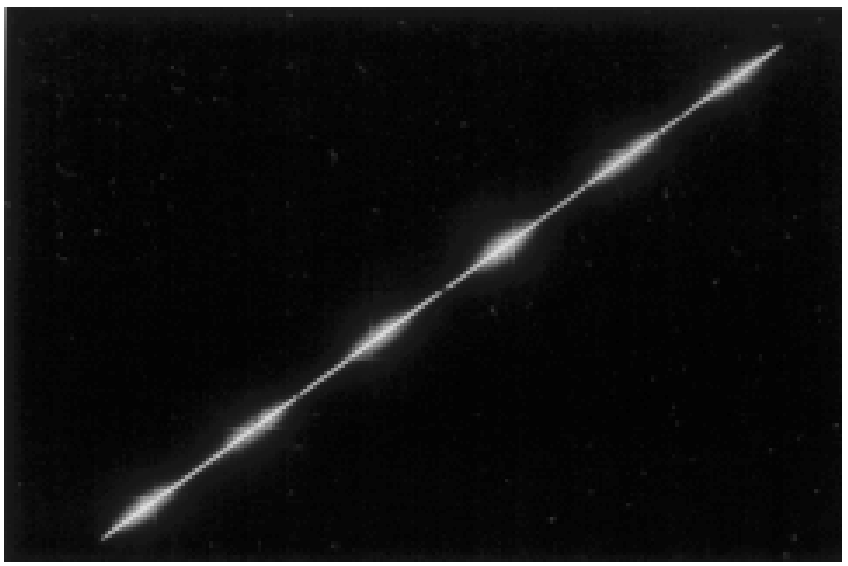

(b) Synchronous when $R_{E}=0.7 \mathrm{k} \Omega$

Fig. 93. Experimental observation of unidirectionally coupled synchronization between two 6-scroll chaotic attractors [Yu et al., 2004b].

Denote $e_{x}=x_{1}-x_{2}, e_{y}=y_{1}-y_{2}, e_{z}=z_{1}-z_{2}$, $e_{w}=w_{1}-w_{2}$. Then, $g\left(y_{1}-x_{1}\right)-g\left(y_{2}-x_{2}\right)=$ $m\left(e_{y}-e_{x}\right)$, where $-0.9=m_{a} \leq m \leq m_{b}=2.9$. Thus, one can recast the error system as follows:

$$
\dot{\mathbf{e}}=(\mathbf{A}-\mathbf{K}+\mathbf{M}) \mathbf{e},
$$

where $\mathbf{e}=\left(e_{x}, e_{y}, e_{z}, e_{w}\right)^{T}$, and

$$
\begin{aligned}
\mathbf{A} & =\left(\begin{array}{cccc}
0 & 0 & -\alpha & 0 \\
0 & 0 & 0 & -\beta \\
1 & 0 & 1 & 0 \\
0 & \gamma & 0 & 0
\end{array}\right), \\
\mathbf{K} & =\left(\begin{array}{cccc}
\delta_{x} & 0 & 0 & 0 \\
0 & \delta_{y} & 0 & 0 \\
0 & 0 & \delta_{z} & 0 \\
0 & 0 & 0 & \delta_{w}
\end{array}\right),
\end{aligned}
$$

$$
\mathbf{M}=\left(\begin{array}{cccc}
-\alpha m & \alpha m & 0 & 0 \\
\beta m & -\beta m & 0 & 0 \\
0 & 0 & 0 & 0 \\
0 & 0 & 0 & 0
\end{array}\right)
$$

Assume that $\alpha=2, \beta=20, \gamma=1.5, m_{0}=$ $m_{2}=0.9, m_{4}=-0.8, m_{1}=m_{3}=m_{5}=2.9$, $c_{1}=1, c_{2}=3.1, c_{3}=5.4, c_{4}=8.193, c_{5}=12.0019$. Yu et al. [2004b] introduced the following unidirectionally coupled synchronization conditions.

Lemma 2 [Yu et al., 2004b]. If there exists a positive definite symmetric constant matrix $\boldsymbol{P}$ such that

$$
(\boldsymbol{A}-\boldsymbol{K}+\boldsymbol{M})^{T} \boldsymbol{P}+\boldsymbol{P}(\boldsymbol{A}-\boldsymbol{K}+\boldsymbol{M}) \leq \mu \boldsymbol{I}<0
$$

uniformly for all $x_{1}, x_{2}, y_{1}, y_{2}$ in the phase space, where $\boldsymbol{I}$ is the identity matrix and $\mu<0$, then the error system (99) is globally exponentially stable about zero. 
For example, when $\delta_{x}=5, \delta_{y}=60, \delta_{z}=60$, $\delta_{w}=6$, one can select $\mathbf{P}=\operatorname{diag}\{0.15,0.15,0.15,0.15\}$ and $\mu=-0.2$ to satisfy the conditions of Lemma 2 . Figure 94 [Yu et al., 2004b] displays the numerical simulation results.

\subsection{Nonlinear $H_{\infty}$ synchronization of n-scroll chaotic attractors}

Yalcin et al. [1999b] experimentally confirmed a nonlinear $H_{\infty}$ synchronization scheme for 5scroll chaotic attractors. Here, the synchronization

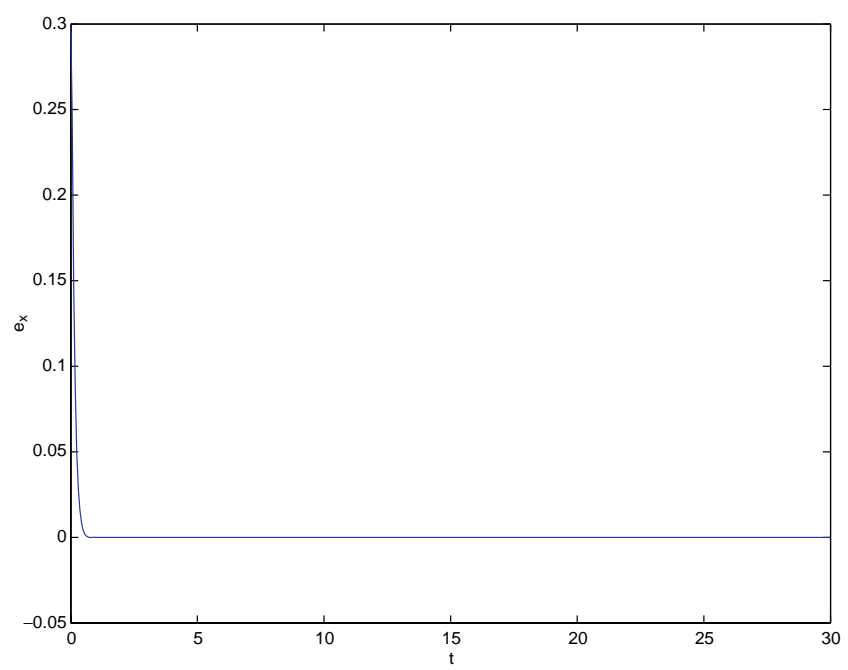

(a) $e_{x}$

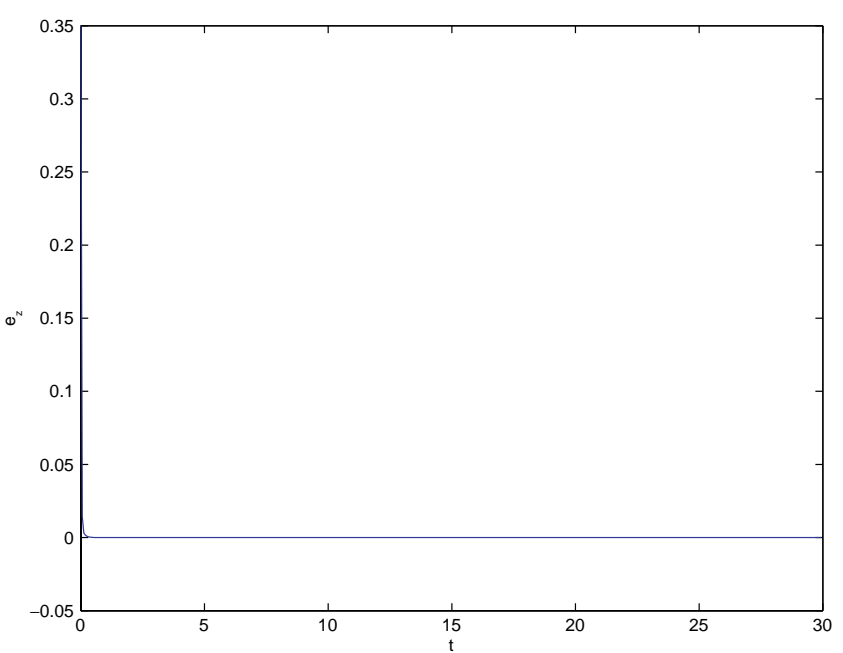

(c) $e_{z}$ scheme has a master-slave configuration with vector field modulation of the master system by the message signal, as shown in Fig. 95, which is described by

$$
\left\{\begin{aligned}
\mathcal{M}: \dot{\mathbf{X}}_{t}= & \mathbf{A}_{t} \mathbf{X}_{t}+\mathbf{B}_{t} \sigma\left(\mathbf{C X}_{t}\right)+\mathbf{D} s(t) \\
\mathcal{S}_{c}: \dot{\mathbf{X}}_{r}= & \mathbf{A}_{r} \mathbf{X}_{r}+\mathbf{B}_{r 1} \sigma\left(\mathbf{C X}_{r}\right) \\
& +\mathbf{B}_{r 2} \sigma\left(\mathbf{C X}_{t}\right)+\mathbf{K X}_{t}
\end{aligned}\right.
$$

where $\mathbf{X}_{t}=\left(x_{t}, y_{t}, z_{t}\right)^{T}=\left(V_{C_{2}}, V_{C_{1}}, I_{L}\right)^{T}$ and $\mathbf{X}_{r}=\left(x_{r}, y_{r}, z_{r}\right)^{T}=\left(\hat{V}_{C_{2}}, \hat{V}_{C_{1}}, \hat{I}_{L}\right)^{T}$ are the state vectors of the master system $\mathcal{M}$ and the slave system $\mathcal{S}_{c}$, respectively,

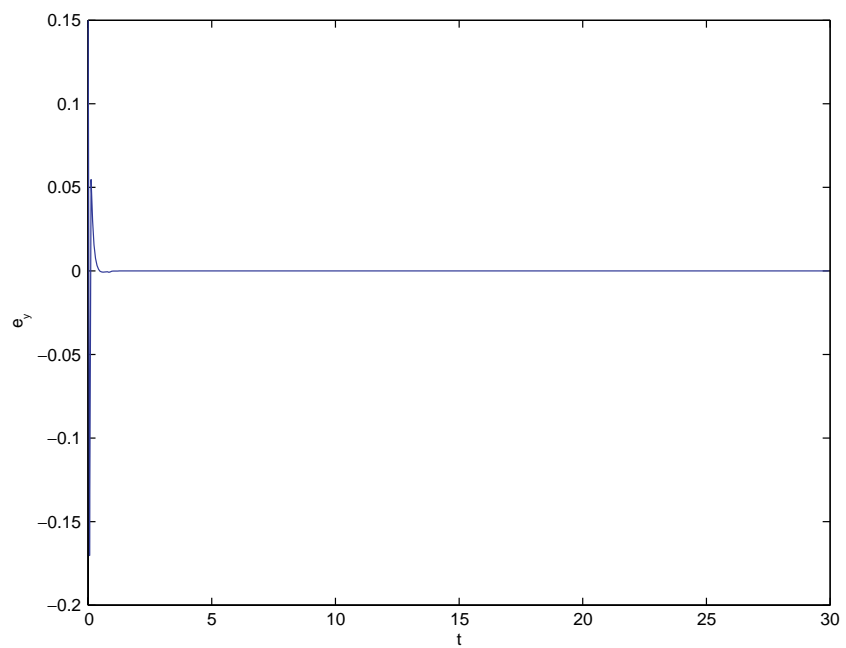

(b) $e_{y}$

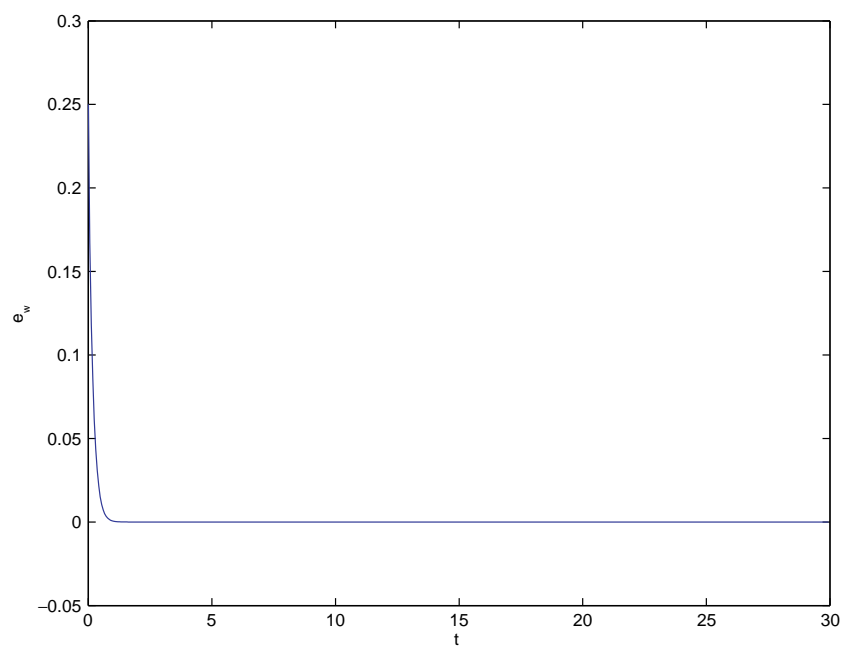

(d) $e_{w}$

Fig. 94. Numerical simulations of unidirectionally coupled synchronization between two 6-scroll hyperchaotic attractors. 


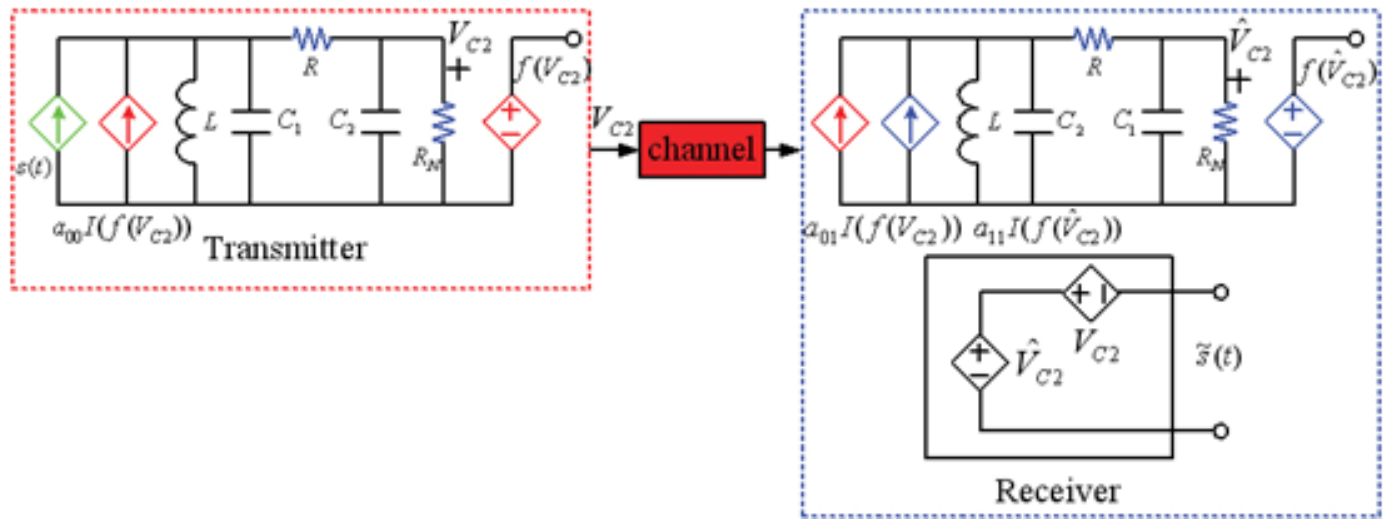

Fig. 95. Circuit implementation scheme for the generalized 5-scroll Chua's circuit.

$$
\begin{gathered}
\mathbf{A}_{t}=\left(\begin{array}{ccc}
-\alpha(1+\delta)+a_{00} \alpha m_{5} & \alpha & 0 \\
1 & -1 & 1 \\
0 & -\beta & 0
\end{array}\right), \quad \mathbf{A}_{r}=\left(\begin{array}{ccc}
-\alpha(1+\delta)+a_{11} \alpha m_{5} & \alpha & 0 \\
1 & -1 & 1 \\
0 & -\beta & 0
\end{array}\right) \\
\mathbf{B}_{t}=\alpha a_{00}\left(\begin{array}{ccc}
\left(m_{0}-m_{1}\right) & 0 & 0 \\
\left(m_{1}-m_{2}\right) & 0 & 0 \\
\left(m_{2}-m_{3}\right) & 0 & 0 \\
\left(m_{3}-m_{4}\right) & 0 & 0 \\
\left(m_{4}-m_{5}\right) & 0 & 0
\end{array}\right), \quad \mathbf{B}_{r 1}=\alpha a_{11}\left(\begin{array}{ccc}
\left(m_{0}-m_{1}\right) & 0 & 0 \\
\left(m_{1}-m_{2}\right) & 0 & 0 \\
\left(m_{2}-m_{3}\right) & 0 & 0 \\
\left(m_{3}-m_{4}\right) & 0 & 0 \\
\left(m_{4}-m_{5}\right) & 0 & 0
\end{array}\right) \\
\mathbf{C}=\left(\begin{array}{lll}
1 & 0 & 0 \\
1 & 0 & 0 \\
1 & 0 & 0 \\
1 & 0 & 0 \\
1 & 0 & 0
\end{array}\right), \quad\left(\begin{array}{ccc}
\left(m_{0}-m_{1}\right) & 0 & 0 \\
\left(m_{1}-m_{2}\right) & 0 & 0 \\
\left(m_{2}-m_{3}\right) & 0 & 0 \\
\left(m_{3}-m_{4}\right) & 0 & 0 \\
\left(m_{4}-m_{5}\right) & 0 & 0
\end{array}\right)
\end{gathered}
$$

$\mathbf{D}=(1,0,0)^{T}$, and $\sigma=\left(\sigma_{1}, \sigma_{2}, \sigma_{3}, \sigma_{4}, \sigma_{5}\right)^{T}$ with $\sigma_{i}(x)=1 / 2\left(\left|x+c_{i}\right|-\left|x-c_{i}\right|\right)$ for $1 \leq i \leq 5$.

Let the system parameters be $\alpha=18, \beta=$ $28.56, \delta=1, m=(1.7089,2.5776,1.7180,2.5777$, $1.6923), c=(0.5305,0.9000,2.0526,3.1875,4.5)$, $a_{00}=1$. Assume that $a_{01}=a_{00}-a_{11}, \mathbf{E}=\mathbf{X}_{t}-\mathbf{X}_{r}$, and the message signal $s(t)$ is binary valued. Then, the original message is recovered as

$$
\tilde{s}(t)=\operatorname{sign}\left(\mathbf{C}_{c} \mathbf{E}\right)
$$

with $\mathbf{C}_{c}=(1,0,0)$.

Therefore, the error system is

$$
\dot{\mathbf{E}}=\mathbf{A} \mathbf{E}+\mathbf{B}\left(\sigma\left(\mathbf{C X}_{t}\right)-\sigma\left(\mathbf{C X}_{r}\right)\right)+\mathbf{D} s,
$$

where $\mathbf{B}=\mathbf{B}_{r 1}$, and

$$
\mathbf{A}=\left(\begin{array}{ccc}
-\alpha(1+\delta)+a_{11} \alpha m_{5} & \alpha & 0 \\
1 & -1 & 1 \\
0 & -\beta & 0
\end{array}\right)
$$

Rewrite (101) as follows:

$$
\dot{\mathbf{E}}=\mathbf{A E}+\mathbf{B} \eta\left(\mathbf{C E}, \mathbf{C X}_{r}\right)+\mathbf{D} s,
$$

where $\eta$ satisfies the sector condition $[0,1]$ and $\eta^{T} \Lambda(\eta-\mathbf{C E}) \leq 0, \forall \mathbf{E}, \mathbf{X}_{r}$, in which $\Lambda$ is a diagonal matrix with positive diagonal elements. Thus, the synchronization scheme is recast in a standard form with tracking error defined by

$$
v(t)=\mathbf{T E}(t)-s(t),
$$

where $\mathbf{T}=\mathbf{C}_{c}$ and the message signal $s(t)$ is considered as an exogenous input. 
Assume that $q(s, v)=\gamma^{2} s^{2}-v^{2}$ and $\phi(\mathbf{E})=$ $\mathbf{E}^{T} \mathbf{P E}$ with $\mathbf{P}=\mathbf{P}^{T}>0$. Then, the dissipativity with a finite $L_{2}$-gain $\gamma$ is obtained if

$$
\dot{\phi} \leq q(s, v), \quad \forall s, v .
$$

Moreover, $\dot{\phi}-q(s, v)-2 \eta^{T} \Lambda(\eta-\mathbf{C E})<0$ which is expressed as a quadratic form in $(e, \eta, s)$. Consequently,

$$
\begin{aligned}
\mathbf{Z} & =\left(\begin{array}{ccc}
\mathbf{A}^{T} \mathbf{P}+\mathbf{P A}+\mathbf{T}^{T} \mathbf{T} & \mathbf{P B}+\mathbf{C}^{T} \Lambda & \mathbf{P D}-\mathbf{T}^{T} \\
\mathbf{B}^{T} \mathbf{P}+\Lambda \mathbf{C} & -2 \Lambda & 0 \\
\mathbf{D}^{T} \mathbf{P}-\mathbf{T} & 0 & -\gamma^{2}+1
\end{array}\right) \\
& <0 .
\end{aligned}
$$

This means that the maximal eigenvalue of $\mathbf{Z}=\mathbf{Z}^{T}$ should be negative.

Thus, the nonlinear $H_{\infty}$ synchronization consists of minimizing the $L_{2}$ gain $\gamma$ as follows:

$$
\min _{a_{01}, \mathbf{Q}, \Lambda, \gamma} \gamma^{2} \text { such that } \mathbf{Z}\left(a_{01}, \mathbf{Q}, \Lambda, \gamma\right)<0,
$$

where $\mathbf{P}=\mathbf{Q}^{T} \mathbf{Q}$.

Yalcin et al. [1999b] also experimentally verified the nonlinear $H_{\infty}$ synchronization using 5 -scroll chaotic attractors, as shown in Fig. 96 [Yalcin et al., 1999b].

\subsection{Controlling n-scroll Chua's circuit}

Zou et al. [2003] introduced a simple proportional and differential (PD) controller to control the $n$ scroll Chua's circuit to a stable equilibrium point or a stable periodic orbit.

When $\alpha=10.814, \beta=14.0, a=1.3, b=0.11$, system (23) generates a 8-scroll chaotic attractor, as shown in Fig. 23(a). Obviously, the equilibrium points of (23) are $\left(x_{E}, 0, x_{E}\right)$, where $x_{E}=2 a k$ and $k=0, \pm 1, \ldots, \pm c$. According to the characteristics of the eigenvalues of the system Jacobi matrix at the equilibria, one can classify the equilibria into two types as follows:

(1) Type I: when $n$ is even and $k$ is odd with $k \leq|c|$, or when $n$ is odd and $k$ is even with $k \leq|c|$, the corresponding eigenvalues of this kind of equilibrium points are one negative real root and a pair of complex conjugate roots with positive real parts.

(2) Type II: when $n$ is even and $k$ is even with $k \leq|c|$, or when $n$ is odd and $k$ is odd with $k \leq|c|$, the corresponding eigenvalues of this kind of equilibrium points are one positive real root and a pair of complex conjugate roots with negative real parts.

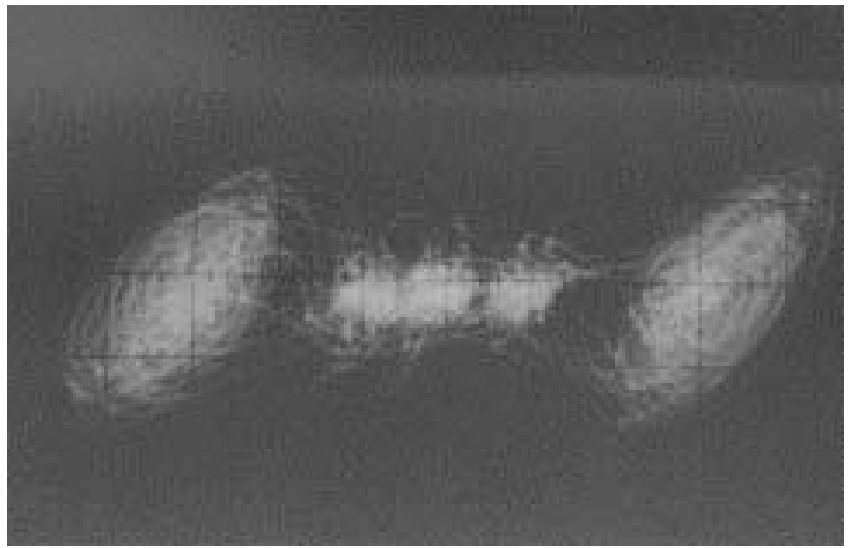

(a) Transmitter $\left(V_{C_{1}}(t), V_{C_{2}}(t)\right)(0.1 \mathrm{~V} / \operatorname{div}-50 \mathrm{mv} / \operatorname{div})$

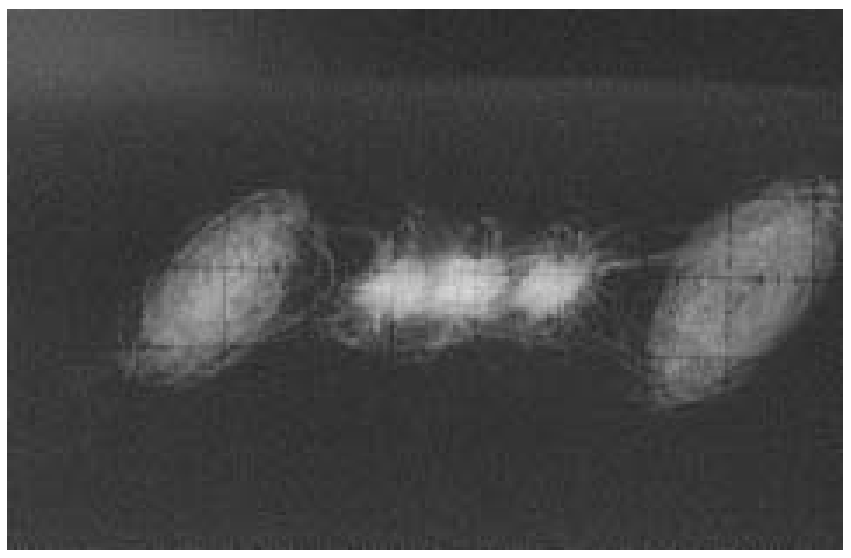

(b) Receiver $\left(\hat{V}_{C_{1}}(t), \hat{V}_{C_{2}}(t)\right)(0.2 \mathrm{~V} / \mathrm{div}-50 \mathrm{mv} / \mathrm{div})$

Fig. 96. Nonlinear $H_{\infty}$ synchronization using 5-scroll attractors [Yalcin et al., 1999b].

Design a controller $u=k_{1} \dot{x}$, where $k_{1}$ is an adjustable parameter, and add it to the second equation of (23). Then, the controlled system becomes

$$
\left\{\begin{array}{l}
\dot{x}=\alpha(y-f(x)) \\
\dot{y}=x-y+z+u \\
\dot{z}=-\beta y
\end{array}\right.
$$

where

$$
f(x)= \begin{cases}\frac{b \pi}{2 a}(x-2 a c) & x \geq 2 a c \\ -b \sin \left(\frac{\pi x}{2 a}+d\right) & -2 a c<x<2 a c \\ \frac{b \pi}{2 a}(x+2 a c) & x \leq-2 a c .\end{cases}
$$


Theoretical analysis shows that the equilibria of type I can be stabilized by using the above controller $u$; however, the equilibria of type II cannot be stabilized by using the same controller $u$. For the equilibria of Type I, the corresponding characteristic equations of the controlled system (102) is

$$
\begin{aligned}
\lambda^{3}+ & \left(2.4373-10.814 k_{1}\right) \lambda^{2} \\
& +4.6233 \lambda+20.1226=0 .
\end{aligned}
$$

According to the Routh-Hurwitz criterion, the equilibria of type I can be stabilized for $k_{1}<$ -0.1771 . If the controller is turned on as soon as the orbit moves into the subspace of the $i$ th scroll, then the orbit can be stabilized to the equilibria in the subspace of the $i$ th scroll.

Furthermore, if the complex conjugate roots of (103) satisfy the following conditions:

$$
\begin{gathered}
\left.\operatorname{Re}(\lambda)\right|_{k_{1}=k_{0}}=0,\left.\quad \operatorname{Im}(\lambda)\right|_{k_{1}=k_{0}} \neq 0, \\
\left.\frac{d \lambda}{d k_{1}}\right|_{k_{1}=k_{0}} \neq 0,
\end{gathered}
$$

where $k_{0}$ is a critical value, then the controlled system (102) has a Hopf bifurcation. For $k_{0}=-0.1771$, the above conditions are satisfied and Hopf bifurcation is generated, as shown in Fig. 97. Figure 98 shows the numerical simulation results of the controlled 8-scroll Chua's circuit.

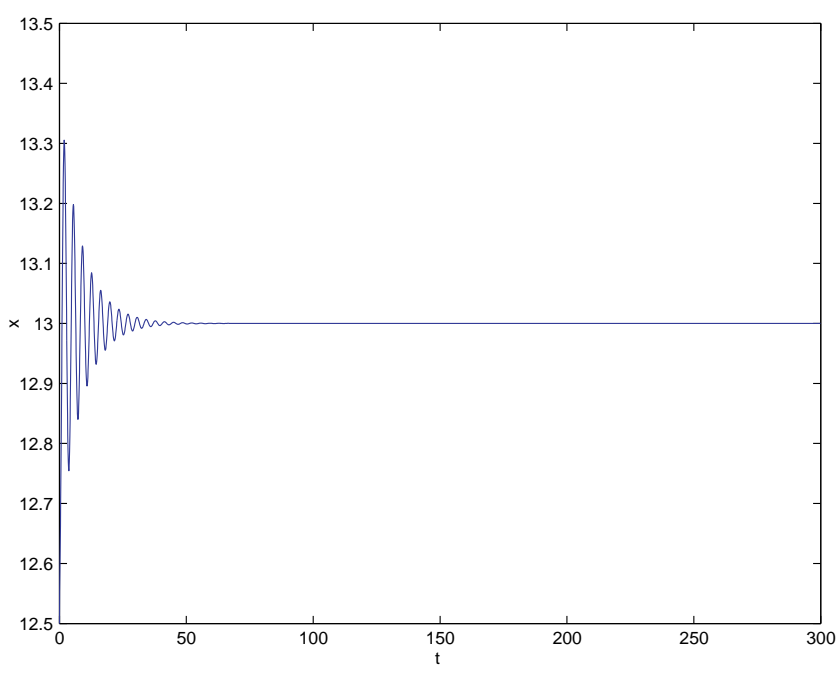

(a) The stabilized equilibrium point $(13,0,-13)$ with $k_{1}=-0.4$

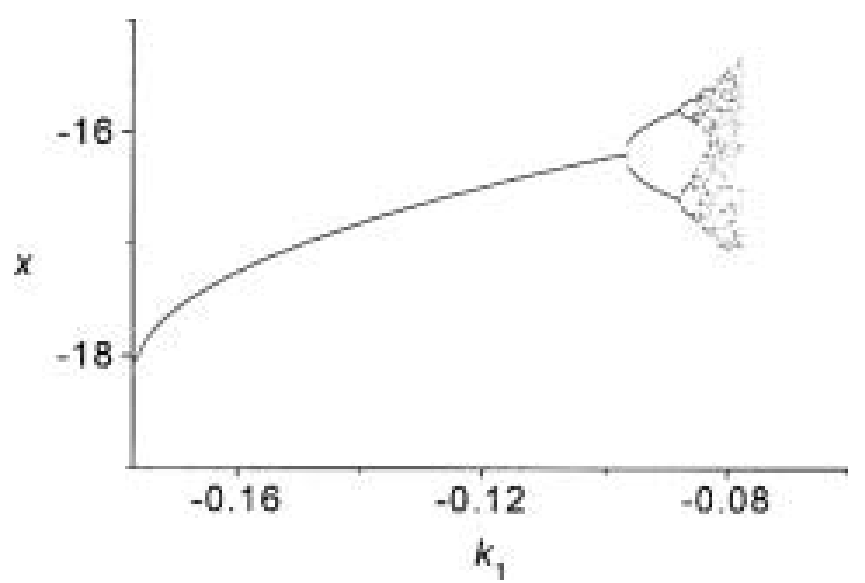

Fig. 97. Period doubling bifurcation for the controlled 8scroll Chua's circuit with $-20<x<-15$.

\subsection{Recurrent neural state-space model for Chua's double-scroll chaotic attractor}

Suykens and Vandewalle [1995] proposed a simple discrete-time autonomous neural state-space model for Chua's circuit that can generate a double-scroll chaotic attractor.

Consider $\mathbf{x}_{k+1}=f\left(\mathbf{x}_{k}\right)$ with state vector $\mathbf{x}_{k} \in$ $\mathbf{R}^{n}$, where $f$ is a continuous function. Parameterizing $f$, by using a multilayer feedforward neural network with one hidden layer, yields

$$
\hat{\mathbf{x}}_{k+1}=\mathbf{W} \tanh \left(\mathbf{V} \hat{\mathbf{x}}_{k}+\beta\right), \quad \hat{\mathbf{x}}_{0}=\mathbf{x}_{0},
$$

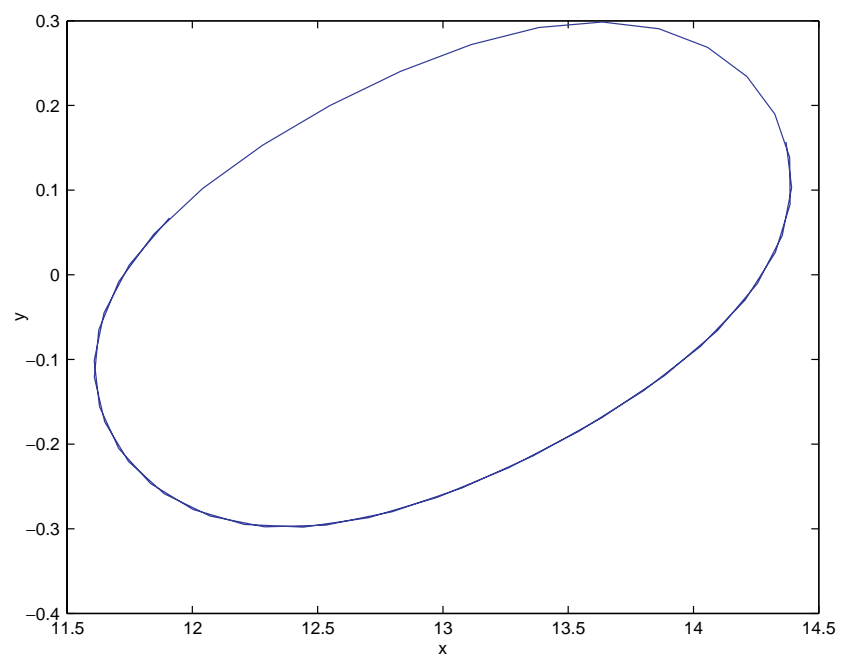

(b) The stabilized period-1 orbit with $k_{1}=-0.4$

Fig. 98. Numerical simulations of the controlled 8-scroll Chua's circuit. 
where the interconnection matrices $\mathbf{W} \in \mathbf{R}^{n \times n_{h}}$, $\mathbf{V} \in \mathbf{R}^{n_{h} \times n}$, and bias vector $\beta \in \mathbf{R}^{n_{h}}$, with $n_{h}$ being the number of hidden neurons. Denote (104) as $\hat{\mathbf{x}}_{k+1}=\Phi\left(\hat{\mathbf{x}}_{k}, \alpha\right)$, where $\alpha$ is an element of the unknown parameter vector $\theta=(\mathbf{W}(:), \mathbf{V}(:), \beta)$ with "(:)" a columnwise scanning of a matrix to a vector.

Minimize the cost function offline, as follows:

$$
\min _{\theta} J(\theta)=\frac{1}{2 N} \sum_{k=1}^{N}\left(\mathbf{x}_{k}-\hat{\mathbf{x}}_{k}(\theta)\right)^{T}\left(\mathbf{x}_{k}-\hat{\mathbf{x}}_{k}(\theta)\right) .
$$

The steepest descent algorithm

$$
\hat{\theta}_{t+1}=\hat{\theta}_{t}-\eta \frac{\partial J}{\partial \theta}
$$

is called dynamic backpropagation. Here, $\eta$ is the learning rate and $\hat{\theta}_{t}$ is the $t$ th iterate.

Consider Chua's circuit (1)-(2), where parameters $C_{1}=1 / 9, C_{2}=1, L=1 / 7, R=10 / 7$, $m_{0}=-0.8, m_{1}=-0.5, b_{1}=1$. Moreover, the time step $h=0.05$ and the initial state $\mathbf{x}_{0}=$ $(0.9365,-0.0610,-0.1889)^{T}$. The training set to be tracked consists of the first 1000 data points. Here, the neural state-space model has three hidden neurons and zero bias vector $\beta$.

Then, an ad hoc optimization procedure is carried out:

(1) Create a random initial parameter vector $\theta_{0}$, and set $a:=1$.

(2) Do, while $a<a_{\text {final }}$,

$$
\begin{aligned}
-\theta^{*} & =\arg \min _{\theta} J(\theta) \\
& =\frac{1}{2 a \Delta} \sum_{k=1}^{a \Delta}\left(\mathbf{x}_{k}-\hat{\mathbf{x}}_{k}\right)^{T}\left(\mathbf{x}_{k}-\hat{\mathbf{x}}_{k}\right) \\
-\theta_{0} & =\theta^{*} \quad \text { and } \quad a:=a+1 .
\end{aligned}
$$

Here, $\Delta=50, a_{\text {final }}=20, \theta_{0}$ is the starting point for the optimization problem, and $\theta^{*}$ is the local optimal solution.

The neural state-space model is obtained as follows:

$$
\begin{gathered}
\mathbf{W}=\left(\begin{array}{rrr}
3.191701795026490 e+00 & -3.961031505875602 e+00 & -2.544300729387972 e+00 \\
6.302937463967251 e-01 & 2.746315947131907 e+00 & 8.024248038305574 e-01 \\
-1.411085817901605 e+00 & 8.436161546347900 e+00 & 3.174868294146957 e+00
\end{array}\right) . \\
\mathbf{V}=\left(\begin{array}{rrr}
-2.446514424620466 e-01 & 1.557093499163188 e+00 & -6.192223155626144 e-01 \\
4.935534636051888 e-01 & -9.111907179654775 e-01 & 6.785493890595847 e-01 \\
-1.711046461663226 e+00 & 3.794433297552783 e+00 & -2.105183558255941 e+00
\end{array}\right) .
\end{gathered}
$$

Figure 99 [Suykens \& Vandewalle, 1995] shows a simple recurrent neural network emulator for generating a double-scroll attractor using the neural state-space model. Obviously, the error becomes larger behind the vertical line due to the nature of the underlying chaotic system.

\subsection{True random bit generation via a double-scroll chaotic attractor}

Yalcin et al. [2002a, 2004a, 2004b] presented a novel "true random bit generator" (TRBG) based on a double-scroll chaotic attractor.

Consider the multiscroll system (31)-(32). When $a=0.8, M_{x}=1, N_{x}=0$, this system can generate a double-scroll chaotic attractor. For given parameters $c_{1}, c_{2}$, the state space of the above double-scroll attractor is divided into three subspaces denoted by $V_{0}=\left\{(x, y, z) \mid x \leq c_{2}\right\}$,
$V_{T}=\left\{(x, y, z) \mid c_{1}<x<c_{2}\right\}$, and $V_{1}=$ $\left\{(x, y, z) \mid x \geq c_{1}\right\}$. Figure 100 [Yalcin et al., 2004a, $2004 \mathrm{~b}]$ shows the discretizing state space for $c_{1}=0$ and $c_{2}=-1$. Here, the block $S_{1}$ is described by

$$
\begin{aligned}
\sigma^{1}(x(t)) & = \begin{cases}0 & \text { if } x(t)<c_{1} \\
1 & \text { if } x(t) \geq c_{1}\end{cases} \\
S_{1}: & \begin{cases}0 & \text { if } x(t)>c_{2} \\
1 & \text { if } x(t) \leq c_{2} .\end{cases}
\end{aligned}
$$

The bit generator block $S_{2}$ is the first block in the software part, which is described by

$$
S_{2}: \sigma_{i}\left(\sigma^{0}, \sigma^{1}\right)=\left\{\begin{array}{lll}
0 & \text { if } \sigma^{0}=0, & \sigma^{1}: 0 \uparrow^{1} \\
1 & \text { if } \sigma^{1}=0, & \sigma^{0}: 0 \uparrow^{1},
\end{array}\right.
$$

where $\sigma^{0}: 0 \uparrow^{1}$ is a raising edge of $\sigma^{0}$ and $i \in$ $\{0,1,2, \ldots\}$. In fact, the bit generation approach aims at characterizing the jumps in signal $x(t)$, either from one scroll to the other, or staying in the same scroll. 


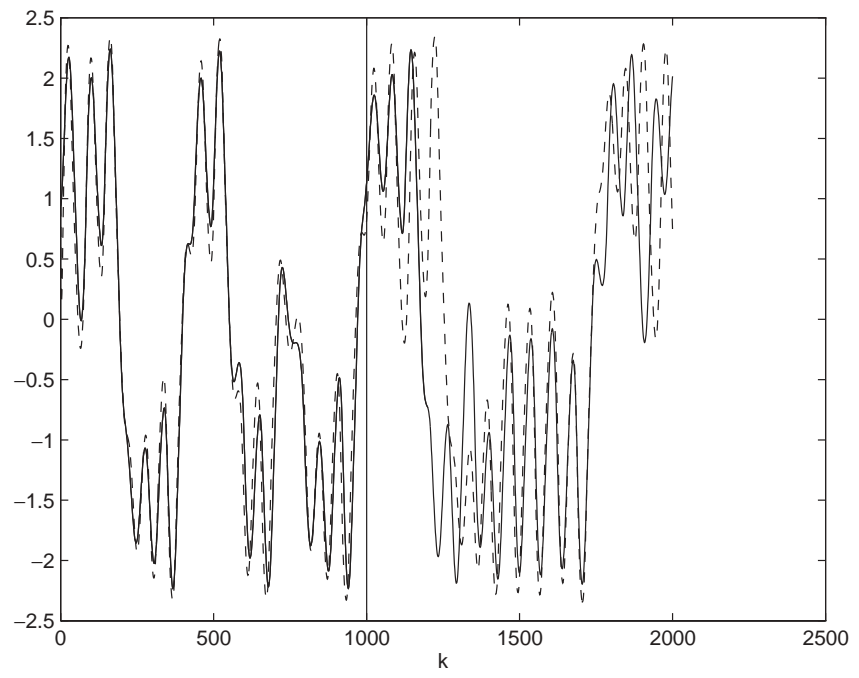

(a) $x$ variable of Chua's circuit and its emulating series

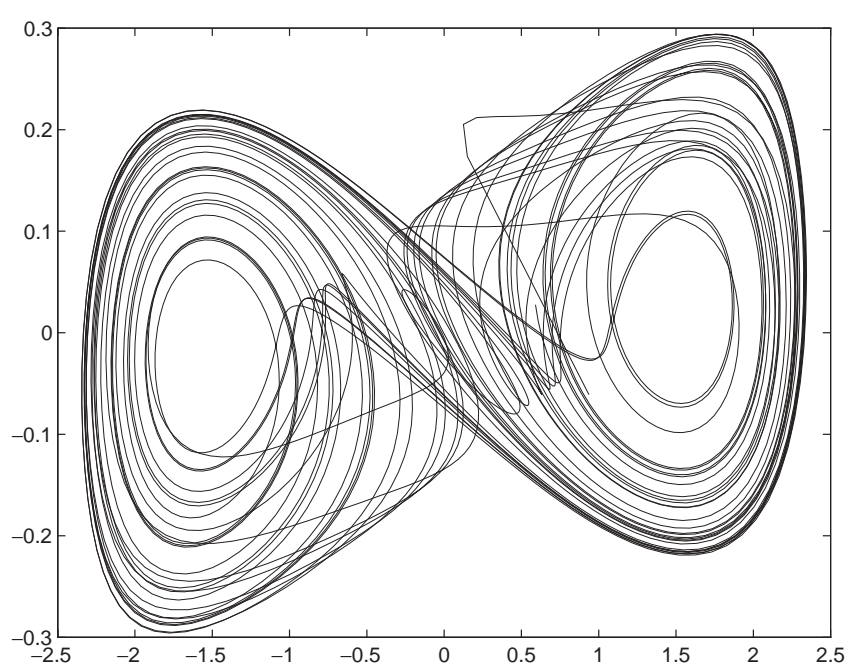

(b) Reconstructed double-scroll attractor $\left(x_{k}, y_{k}\right)$

Fig. 99. A simple recurrent neural network emulator for Chua's circuit [Suykens \& Vandewalle, 1995].

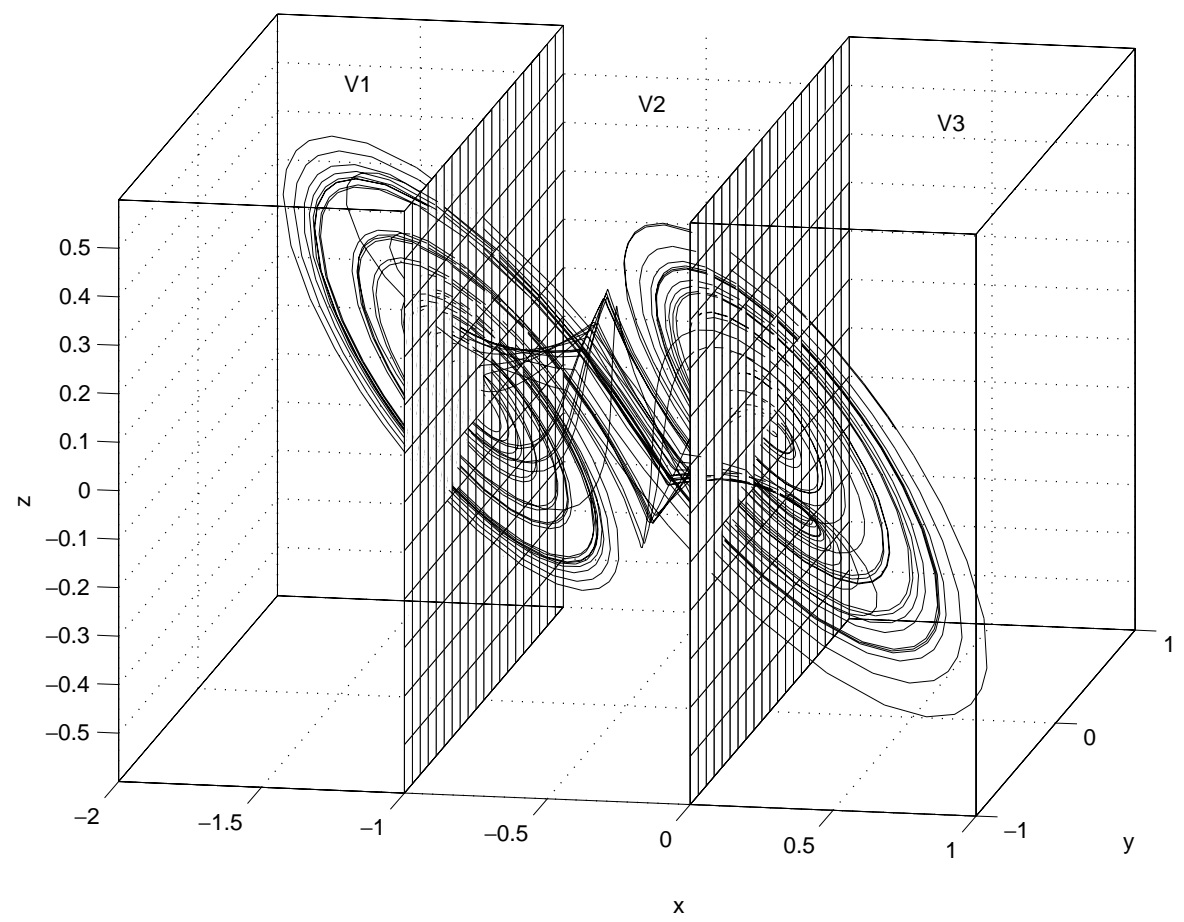

Fig. 100. Discretizing the state space for $c_{1}=0$ and $c_{2}=-1$ [Yalcin et al., 2004a, 2004b].

The de-skewing block $S_{3}$ is described by

$$
S_{3}: b_{i}\left(\sigma_{i}, \sigma_{i-1}\right)= \begin{cases}0 & \text { if } \sigma_{i}=0 \wedge \sigma_{i-1}=1 \\ 1 & \text { if } \sigma_{i}=1 \wedge \sigma_{i-1}=0 .\end{cases}
$$

The above-proposed TRBG has a single output denoted by $\mathcal{B}=\{\ldots, b(i-1), b(i), b(i+1), \ldots\}$, with $b(i) \in\{0,1\}$. Since noise has maximum entropy, the threshold value $c_{2}$ is chosen such that the measured entropy of the TRBG is maximal. The measuredtheoretical entropy of the proposed TRBG with respect to a partition $c_{2}$ is given by

$$
h^{c_{2}}=\lim _{n \rightarrow \infty} \frac{H_{n}^{c_{2}}}{n},
$$

where

$$
H_{n}^{c_{2}}=-\sum_{\mathcal{B}^{n}} P\left(\mathcal{B}^{n}\right) \ln P\left(\mathcal{B}^{n}\right)
$$




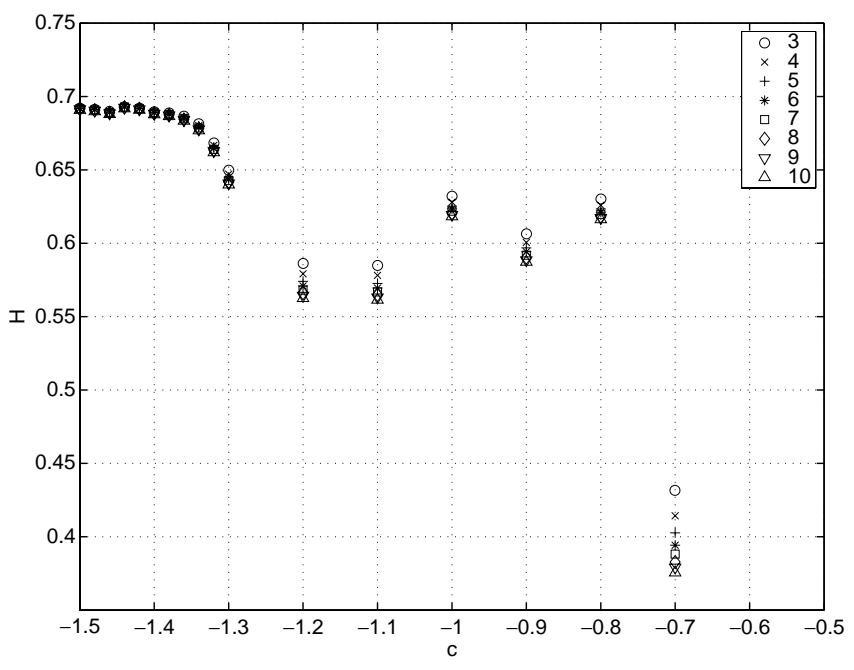

Fig. 101. $H_{n}^{c_{2}} / n$ as a function of the threshold $c_{2}$ for $n=$ 3, .., 10 [Yalcin et al., 2004a, 2004b].

with $P\left(\mathcal{B}^{n}\right)$ being the probability of occurrence of the binary subsequence (words $\{\ldots,\{b(i), b(i+$ $1), \ldots, b(i+n-1)\},\{b(i+n), \ldots, b(i+2 n-1)\}, \ldots\})$ of length $n . H_{n}^{c_{2}}$ achieves its maximum value when the $n$-word sequences in the output sequence $\mathcal{B}$ of the TRBG are equally distributed.

Figure 101 [Yalcin et al., 2004a, 2004b] displays the values of $H_{n}^{c_{2}} / n$ for $n=3,4, \ldots, 10$, and also shows how the TRBG can approach the maximum entropy $\ln 2$. The threshold value $c_{1}$ is set to 0 Volt and that of $c_{2}$ is set to -1.44 Volt. Yalcin et al. [2004a, 2004b] also experimentally verified the randomness of the TRBG by using the well-known test set FIPS-140-1 and Diehard in cryptography.

\section{Conclusion and Discussion}

This paper has surveyed the recent main advances in multiscroll chaotic attractors generation, including some fundamental theories, design approaches, and potential applications. Basic theoretical design approaches developed over the last decade utilized PWL functions, CNNs, nonlinear modulating functions, fundamental circuits, switching manifolds, multifolded torus forming, and so on. The dynamical mechanism of multiscroll chaotic attractors in the aforementioned methods is now well understood, which can even be applied to designing various multidirectional multiscroll chaotic or hyperchaotic attractors.

In general, multiscroll chaotic attractors are verified by numerical simulations, but lately this has seen improvement in terms of theoretical proofs and analog circuit realization. Despite the physical difficulties and limitations, today one is able to physically implement up a maximum of $1-\mathrm{D} 14$ scroll, 2 -D $14 \times 10$-grid scroll and 3 -D $10 \times 10 \times 10$ grid (1000) scroll chaotic attractors by electronic circuits. It remains a technical challenge to produce more scrolls via hardware implementation, though numerical simulations can do much better. On the other hand, rigorous theoretical proofs are also quite difficult due to the complex dynamical behaviors and the lack of suitable mathematical tools, especially for switching systems. Therefore, to physically realize and also theoretically prove the existence of chaotic attractors with a multidirectional orientation and a large number of scrolls appears to be an important and stimulating subject for future research.

It can be foreseen that multiscroll chaotic attractors will have many unusual practical applications in such fields as digital and secure communications, synchronous prediction, random bit generation, information systems, and so on. Exploring the promising potential of multiscroll chaotic attractors for engineering applications calls for more efforts and greater endeavors.

In summary, the subject of multiscroll chaotic attractors generation has seen exciting development over the last decade, not only in deeper and wider theoretical studies but also in many newly found real-world applications. It deserves further attention with continued pursuit from the communities of engineering, physics, applied mathematics and electronic technology.

\section{Acknowledgments}

This work was supported by the National Natural Science Foundation of China under Grants No.60304017 and No.20336040, the Scientific Research Startup Special Foundation on Excellent $\mathrm{PhD}$ Thesis and Presidential Award of Chinese Academy of Sciences, and the City University of Hong Kong under the SRG Grant 7001702 (EE). The authors would like to thank Profs. Lei Guo, Daizhan Cheng, Xinghuo Yu, Yiguang Hong, Wallace Kit-Sang Tang, Kim-Fung Man, Guo-Qun Zhong, Simin Yu, Fengling Han, among others, for their valuable comments, discussions, and cooperations. The authors especially thank Prof. Simin $\mathrm{Yu}$ for redrawing most of the circuit diagrams and thank Profs. M. A. Aziz-Alaoui, A. S. Elwakil, J. A. K. Suykens, M. E. Yalcin, 
Wallace Kit-Sang Tang, Guo-Qun Zhong, Simin Yu, Wajdi M. Ahmad and Xiaosong Yang for providing their original figures.

Some figures in this review article are taken from published papers. These figures have all been credited to the original authors and publishers, in both the text and the figure captions. The authors of this article sincerely thank the original authors and publishers for their courtesy.

\section{References}

Ahmad, W. M. [2005] "Generation and control of multiscroll chaotic attractors in fractal order systems," Chaos Solit. Fract. 25, 727-735.

Arena, P., Baglio, S., Fortuna, L. \& Manganaro, G. [1995] "Chua's circuit can be generated by CNN cells," IEEE Trans. Circuits Syst.-I 42, 123-125.

Arena, P., Baglio, S., Fortuna, L. \& Manganaro, G. [1996a] "Generation of $n$-double scrolls via cellular neural networks," Int. J. Circuit Th. Appl. 24, 241252.

Arena, P., Baglio, S., Fortuna, L. \& Manganaro, G. [1996b] "State controlled CNN: A new strategy for generating high complex dynamics," IEICE Trans. Fund. E79-A, 1647-1657.

Aziz-Alaoui, M. A. [1999] "Differential equations with multispiral attractors," Int. J. Bifurcation and Chaos 9, 1009-1039.

Aziz-Alaoui, M. A. [2000] "Multispiral chaos," Proc. 2nd Int. Conf. Control of Oscillations and Chaos 1, July 5-7, St. Petersberg, Russsia, pp. 88-91.

Aziz-Alaoui, M. A. [2001] "Dynamics of a HénonLozi-type map," Chaos Solit. Fract. 12, 23232341.

Cafagna, D. \& Grassi, G. [2003a] "Hyperchaotic coupled Chua circuits: An approach for generating new $n \times m$ scroll attractors," Int. J. Bifurcation and Chaos 13, $2537-2550$

Cafagna, D. \& Grassi, G. [2003b] "New 3D-scroll attractors in hyperchaotic Chua's circuit forming a ring," Int. J. Bifurcation and Chaos 13, 28892903.

Chen, G. \& Dong, X. [1998] From Chaos to Order: Methodologies, Perspectives and Applications (World Scientific, Singapore).

Chen, G. \& Lü, J. [2003] Dynamics of the Lorenz System Family: Analysis, Control and Synchronization (in Chinese) (Science Press, Beijing).

Chen, G. \& Yu, X. (eds.) [2003] Chaos Control: Theory and Applications (Springer-Verlag, Berlin, Heidelberg).

Chen, Q., Hong, Y. G. \& Chen, G. [2004] "Generation of chaotic torus attractors via switching control scheme," submitted.
Chen, S. H. \& Lü, J. [2002] "Synchronization of an uncertain unified chaotic system via adaptive control," Chaos Solit. Fract. 14, 643-647.

Chua, L. O., Komuro, M. \& Matsumoto, T. [1986] "The double scroll family," IEEE Trans. Circuits Syst. 33, 1072-1118.

Chua, L. O. [1998] CNN: A Paradigm for Complexity (World Scientific, Singapore).

Eguchi, K., Inoue, T. \& Tsuneda, A. [1999] "Synthesis and analysis of a digital chaos circuit generating multiple-scroll strange attractors," IEICE Trans. Fund. E82-A, 965-972.

Elwakil, A. S. \& Kennedy, M. P. [1999] "Chaotic oscillators derived from Saito's double-screw hysteresis oscillator," IEICE Trans. Fund. E82-A, 17691775 .

Elwakil, A. S. [2000] "Chaotic oscillator configuration using a frequency dependent negative resistor," Int. J. Circuit Th. Appl. 28, 69-76.

Elwakil, A. S. \& Kennedy, M. P. [2000a] "Improved implementation of Chua's chaotic oscillator using current feedback op amp," IEEE Trans. Circuits Syst.-I 47, 76-79.

Elwakil, A. S. \& Kennedy, M. P. [2000b] "Systematic realization of a class of hysteresis chaotic oscillators," Int. J. Circuit Th. Appl. 28, 319-334.

Elwakil, A. S., Salama, K. N. \& Kennedy, M. P. [2000] "A system for chaos generation and its implementation in monolithic form," Proc. IEEE Int. Symp. Circuits and Systems (ISCAS'00) 5, May 28-31, Geneva, Switzerland, pp. 217-220.

Elwakil, A. S. \& Kennedy, M. P. [2001] "Construction of classes of circuit-independent chaotic oscillators using passive-only nonlinear devices," IEEE Trans. Circuits Syst.-I 48, 289-307.

Elwakil, A. S. [2002] "Nonautonomous pulse-driven chaotic oscillator based on Chua's circuit," Microelectronics J. 33, 479-486.

Elwakil, A. S., Özoğuz, S. \& Kennedy, M. P. [2002] "Creation of a complex butterfly attractor using a novel Lorenz-type system," IEEE Trans. Circuits Syst.-I 49, 527-530.

Fujiwara, T., Horio, Y. \& Aihara, K. [2003] "An integrated multi-scroll circuit with floating-gate MOSFETs," Proc. IEEE Int. Symp. Circuits and Systems (ISCAS'03) 3, May 25-28, Bangkok, Thailand, pp. $180-183$.

Han, F., Yu, X., Chen, G., Liu, W. \& Feng, Y. [2003a] "Generation of multi-scroll chaos using second-order linear systems with hysteresis," Proc. IEEE Int. Symp. Circuits and Systems (ISCAS'03) 3, May 2528, Bangkok, Thailand, pp. 84-87.

Han, F., Yu, X., Wang, Y., Feng, Y. \& Chen, G. [2003b] " $n$-scroll chaotic oscillators by second-order systems and double-hysteresis blocks," Electron. Lett. 39, 1636-1638. 
Han, F. [2004] Multi-scroll Chaos Generation via Linear Systems and Hysteresis Function Series, Ph.D thesis, RMIT University, Melbourne, Australia.

Han, F., Lü, J., Yu, X., Chen, G. \& Feng, Y. [2005] "Generating multi-scroll chaotic attractors via a linear second-order hysteresis system," Dyn. Contin. Discr. Impul. Syst. Series B, 12, 95-110.

Inaba, N. [1992] "Folded torus in the forced rayleigh oscillator with a diode pair," IEEE Trans. Circuits Syst. - I 39, 402-411.

Kennedy, M. P. [1993] "Three steps to chaos, Part II: A Chua's circuit primer," IEEE Trans. Circuits Syst.-I 40, 657-674.

Li, Q., Yang, X. \& Yang, F. [2003] "Multiple-scrolls chaotic attractor and circuit implementation," Electron. Lett. 39, 1306-1307.

Linz, S. J. \& Sprott, J. C. [1999] "Elementary chaotic flow," Phys. Lett. A 259, 240-245.

Liu, W. \& Chen, G. [2004] "Can a three-dimensional smooth autonomous quadratic chaotic system generate a single four-scroll attractor?" Int. J. Bifurcation and Chaos 14, 1395-1403.

Lü, J. \& Chen, G. [2002] "A new chaotic attractor coined," Int. J. Bifurcation and Chaos 12, 659-661.

Lü, J., Chen, G., Cheng, D. Z. \& Celikovsky, S. [2002a] "Bridge the gap between the Lorenz system and the Chen system," Int. J. Bifurcation and Chaos 12, 2917-2926.

Lü, J., Chen, G. \& Wang, X. F. [2002b] "Generating chaos via control," Proc. 21th Chinese Control Conf., Zhejiang University Press, August 13-15, Hangzhou, China, pp. 538-542.

Lü, J., Chen, G. \& Yu, Y. [2002c] "Asymptotic analysis of a modified Lorenz system," Chin. Phys. Lett. 19, 1260-1263.

Lü, J., Chen, G. \& Zhang, S. C. [2002d] "Dynamical analysis of a new chaotic attractor," Int. J. Bifurcation and Chaos 12, 1001-1015.

Lü, J., Lu, J. \& Chen, S. [2002e] Chaotic Time Series Analysis and Its Applications (in Chinese) (Wuhan University Press, Wuhan, China).

Lü, J., Zhou, T., Chen, G. \& Yang, X. [2002f] "Generating chaos with a switching piecewise-linear controller," Chaos 12, 344-349.

Lü, J., Zhou, T. \& Zhang, S. C. [2002g] "Chaos synchronization between linearly coupled chaotic system," Chaos Solit. Fract. 14, 529-541.

Lü, J. [2003] "Switching control: From simple rules to complex chaotic systems," J. Syst. Sci. Compl. 16, 404-413.

Lü, J., Yu, X. \& Chen, G. [2003a] "Generating chaotic attractors with multiple merged basins of attraction: A switching piecewise-linear control approach," IEEE Trans. Circuits Syst.-I 50, 198-207.

Lü, J., Yu, X. \& Chen, G. [2003b] "Switching control for multi-scroll chaos generation: An overview," Proc.
Int. Conf. Physics and Control 2, August 20-22, Saint Petersburg, Russia, pp. 420-428.

Lü, J., Chen, G. \& Cheng, D. Z. [2004a] "A new chaotic system and beyond: The general Lorenzlike system," Int. J. Bifurcation and Chaos 14, 1507-1537.

Lü, J., Chen, G., Yu, X. \& Leung, H. [2004b] "Generating multi-scroll chaotic attractors via switching control," Proc. 5th Asian Control Conf., July 20-23, Melbourne, Australia, pp. 1763-1771.

Lü, J., Chen, G., Yu, X. \& Leung, H. [2004c] "Design and analysis of multi-scroll chaotic attractors from saturated function series," IEEE Trans. Circuits Syst.-I 51, 2476-2490.

Lü, J., Han, F., Yu, X. \& Chen, G. [2004e] "Generating 3-D multi-scroll chaotic attractors: A hysteresis series switching method," Automatica 40, 1677-1687.

Lü, J., Yu, S. M. \& Leung, H. [2005a] "Design of 3-D multi-scroll chaotic attractors via basic circuits," Proc. DCDIS 4th Int. Conf. Engineering Applications and Computational Algorithms, July 27-29, Guelph, Ontario, Canada, pp. 324-328.

Lü, J., Yu, S. M., Leung, H. \& Chen, G. [2005b] "Experimental verification for 3-D hysteresis multiscroll chaotic attractors," Proc. IEEE Int. Symp. Circuits and Systems (ISCAS'05), May 23-26, Kobe, Japan, pp. 3391-3394.

Lü, J., Yu, S. M., Leung, H. \& Chen, G. [2006] "Experimental verification of multi-directional multiscroll chaotic attractors," IEEE Trans. Circuits Syst.-I 53, 149-165.

Makoto, I., Yang, T. \& Chua, L. O. [1999] "Experimental study of impulsive synchronization of chaotic and hyperchaotic circuits," Int. J. Bifurcation and Chaos 7, 1393-1424.

Matsumoto, T., Chua, L. O. \& Komuro, M. [1985] "The double scroll," IEEE Trans. Circuits Syst. CAS-32, 798-817.

Matsumoto, T., Chua, L. O. \& Kobayashi, K. [1986] "Hyperchaos: Laboratory experiment and numerical confirmation," IEEE Trans. Circuits Syst. CAS-33, 1143-1147.

Matsumoto, T. [1987] "Chaos in electronic circuits," Proc. IEEE 75, 1033-1057.

Matsumoto, T., Chua, L. O. \& Tokunaga, R. [1987] "Chaos via torus breakdown," IEEE Trans. Circuits Syst. CAS-34, 240-253.

Miranda, R. \& Stone, E. [1993] "The proto-Lorenz system," Phys. Lett. A 178, 105-113.

Nakagawa, S. \& Saito, T. [1996] "An RC OTA hysteresis chaos generator," IEEE Trans. Circuits Syst.-I 43, 1019-1021.

Özoğuz, S., Elwakil, A. S. \& Salama, K. N. [2002] " $n$-Scroll chaos generator using nonlinear transconductor," Electron. Lett. 38, 685-686. 
Saito, T. [1990] "An approach toward higher dimensional hysteresis chaos generators," IEEE Trans. Circuits Syst.-I 37, 399-409.

Saito, T. \& Nakagawa, S. [1995] "Chaos from a hysteresis and switched circuit," Phil. Trans. R. Soc. Lond. A 353, 47-57.

Salama, K. N., Özoğuz, S. \& Elwakil, A. S. [2003] "Generation of $n$-scroll chaos using nonlinear transconductors," Proc. IEEE Int. Symp. Circuits and Systems (ISCAS'03) 3, May 25-28, Bangkok, Thailand, pp. 176-179.

Silva, C. P. [1993] "Shil'nikov's theorem - A tutorial," IEEE Trans. Circuits Syst.-I 40, 675-682.

Storace, M. \& Parodi, M. [1998] "Simple realization of hysteresis chaos generator," Electron. Lett. 34, $10-11$.

Suykens, J. A. K. \& Vandewalle, J. [1991] "Quasilinear approach to nonlinear systems and the design of $n$-double scroll $(n=1,2,3,4, \ldots)$, IEE Proc. $G \mathbf{1 3 8}$, 595-603.

Suykens, J. A. K. \& Vandewalle, J. [1993a] "Generation of $n$-double scrolls $(n=1,2,3,4, \ldots), "$ IEEE Trans. Circuits Syst.-I 40, 861-867.

Suykens, J. A. K. \& Vandewalle, J. [1993b] "Between $n$ double sinks and $n$-double scrolls $(n=1,2,3,4, \ldots)$," Proc. Int. Symp. Nonlinear Theory and Its Applications (NOLTA'93), December 5-10, Hawaii, USA, pp. 829-834.

Suykens, J. A. K. \& Vandewalle, J. [1995] "Learning a simple recurrent neural state space model to behave like Chua's double scroll," IEEE Trans. Circuits Syst.-I 42, 499-502.

Suykens, J. A. K. \& Chua, L. O. [1997] " $n$-Double scroll hypercubes in 1-D CNNs," Int. J. Bifurcation and Chaos 7, 1873-1885.

Suykens, J. A. K., Huang, A. \& Chua, L. O. [1997] "A family of $n$-scroll attractors from a generalized Chua's circuit," Int. J. Electron. Commun. 51, 131-138.

Suykens, J. A. K. \& Vandewalle, J. [1998] "The K. U. Leuven time series prediction competition," in Nonlinear Modeling: Advanced Black-Box Techniques, eds. Suykens, J. A. K. \& Vandewalle, J. (Kluwer Academic Publishers, Boston, USA), pp. 241253.

Tang, K. S., Man, K. F. \& Chen, G. [2001a] "Digitized $n$-scroll attractors model for secure communications," Proc. IEEE Int. Symp. Circuits and Systems (ISCAS'01) 3, May 6-9, Sydney, Australia, pp. 787790.

Tang, K. S., Zhong, G. Q., Chen, G. \& Man, K. F. [2001b] "Generation of $n$-scroll attractors via sine function," IEEE Trans. Circuits Syst.-I 48, 13691372 .

Wada, M., Nishio, Y. \& Ushida, A. [1999] "Chaotic itinerancy phenomena on coupled $n$-double scrolls chaotic circuits," Proc. IEEE Int. Symp. Circuits and Systems (ISCAS'99) 5, May 30-June 2, Orlando, Florida, USA, pp. 487-490.

Yalcin, M. E., Suykens, J. A. K. \& Vandewalle, J. [1999a] "On the realization of $n$-scroll attractors," Proc. IEEE Int. Symp. Circuits and Systems (ISCAS'99) 5, May 30-June 2, Orlando, Florida, USA, pp. 483-486.

Yalcin, M. E., Suykens, J. A. K. \& Vandewalle, J. [1999b] "Experimental confirmation of a nonlinear $H_{\infty}$ synchronization scheme with 5-scroll attractors," Proc. Int. Workshop on Nonlinear Dynamics of Electronic Systems (NDES'g9), July 15-17, Ronne, Denmark, pp. 257-260.

Yalcin, M. E., Suykens, J. A. K. \& Vandewalle, J. [2000a] "Hyperchaotic $n$-scroll attractors," Proc. IEEE Workshop on Nonlinear Dynamics of Electronic systems (NDES'00), May 18-20, Catania, Italy, pp. $25-28$.

Yalcin, M. E., Suykens, J. A. K. \& Vandewalle, J. [2000b] "Experimental confirmation of 3- and 5-scroll attractors from a generalized Chua's circuit," IEEE Trans. Circuits Syst.-I 47, 425-429.

Yalcin, M. E., Özoğuz, S., Suykens, J. A. K. \& Vandewalle, J. [2001] " $n$-scroll chaos generators: A simple circuit model," Electron. Lett. 37, 147-148.

Yalcin, M. E., Özoğuz, S., Suykens, J. A. K. \& Vandewalle, J. [2002a] "Scroll maps from $n$-scroll attractors," Proc. 10th Int. Workshop on Nonlinear Dynamics of Electronic Systems (NDES'O2), June 2123, Izmir, Turkey, pp. 45-48.

Yalcin, M. E., Suykens, J. A. K., Vandewalle, J. \& Ozoguz, S. [2002b] "Families of scroll grid attractors," Int. J. Bifurcation and Chaos 12, 23-41.

Yalcin, M. E. [2004] Cellular Neural Networks, Multiscroll Chaos and Synchronization: Theory, Applications and Implementations, $\mathrm{PhD}$ thesis, Katholieke Universiteit Leuven, Belgium.

Yalcin, M. E., Suykens, J. A. K. \& Vandewalle, J. [2004a] "True random bit generation from a double-scroll attractor," IEEE Trans. Circuits Syst.-I 51, 13951404.

Yalcin, M. E., Suykens, J. A. K. \& Vandewalle, J. [2004b] "A double scroll based true random bit generator," Proceedings of the IEEE International Symposium on Circuits and Systems (ISCAS'04) 4, May 23-26, Vancouver, Canada, pp. 581-584.

Yalcin, M. E., Suykens, J. A. K. \& Vandewalle, J. [2005] Cellular Neural Networks, Multiscroll Chaos and Synchronization (World Scientific, Singapore).

Yang, X. \& Li, Q. [2003] "Generate $n$-scroll attractors in linear system by scalar output feedback," Chaos Solit. Fract. 18, 25-29.

Yin, Y. Z. [1996] "Synchronization of chaos in a modified Chua's circuit using continuous control," Int. J. Bifurcation and Chaos 11, 2101-2117. 
Yu, S. M., Lin, Q. H. \& Qiu, S. S. [2003a] "Simulation investigation on multi-scroll chaotic and hyperchaotic attractors for four-dimensional systems," Acta Phys. Sinica 52, 25-33.

Yu, S. M., Qiu, S. S. \& Lin, Q. H. [2003b] "New results of study on generating multiple-scroll chaotic attractors," Science in China Series F 46, 104-115.

Yu, S. M., Lin, Q. H. \& Qiu, S. S. [2004a] "A family of multiple-folded torus chaotic attractors," Acta Phys. Sin. 53, 2084-2088.

Yu, S. M., Ma, Z. G., Qiu, S. S., Peng, S. G. \& Lin, Q. H. [2004b] "Generation and synchronization of $n$ scroll chaotic and hyperchaotic attractors in fourthorder systems," Chin. Phys. 13, 317-328.

Yu, S. M., Lü, J., Leung, H. \& Chen, G. [2005a] "Design and circuit implementation of $n$-scroll chaotic attractor from a general Jerk system," IEEE Trans. Circuits Syst.-I 52, 1459-1476.

Yu, S. M., Lü, J. \& Chen, G. [2005b] "Circuit and DSP realization of $n$-scroll hyperchaotic attractors," Chaos, submitted.
Yu, S. M., Lü, J., Leung, H. \& Chen, G. [2005c] "Multifolded torus chaos generation: Theoretical design and circuit implementation," IEEE Trans. Circuits Syst.-I, submitted.

Yu, S. M., Lü, J., Leung, H. \& Chen, G. [2005d] " $N$-scroll chaotic attractors from a general Jerk circuit," Proc. IEEE Int. Symp. Circuits and Systems (ISCAS'05), May 23-26, Kobe, Japan, pp. 1473-1476.

Yu, S. M., Lü, J., Leung, H. \& Chen, G. [2005e] "Generating multi-folded torus chaotic attractors," Proc. Int. Conf. Physics and Control, Saint Petersburg, Russia, August 24-26, pp. 249-253.

Zhong, G., Man, K. F. \& Chen, G. [2002] "A systematic approach to generating $n$-scroll attractors," Int. J. Bifurcation and Chaos 12, 2907-2915.

Zou, Y., Luo, X., Jiang, P., Wang, B., Chen, G., Fang, J. \& Quan, H. [2003] "Controlling the chaotic $n$-scroll Chua's circuit," Int. J. Bifurcation and Chaos 13, 2709-2714. 